This item was submitted to Loughborough's Research Repository by the author.

Items in Figshare are protected by copyright, with all rights reserved, unless otherwise indicated.

\title{
Parallel operation of bridge rectifiers without an interbridge reactor
}

PLEASE CITE THE PUBLISHED VERSION

PUBLISHER

Loughborough University of Technology

LICENCE

CC BY-NC 4.0

REPOSITORY RECORD

Razak, Abdul. 2020. "Parallel Operation of Bridge Rectifiers Without an Interbridge Reactor". Loughborough University. https://doi.org/10.26174/thesis.lboro.13079249.v1. 

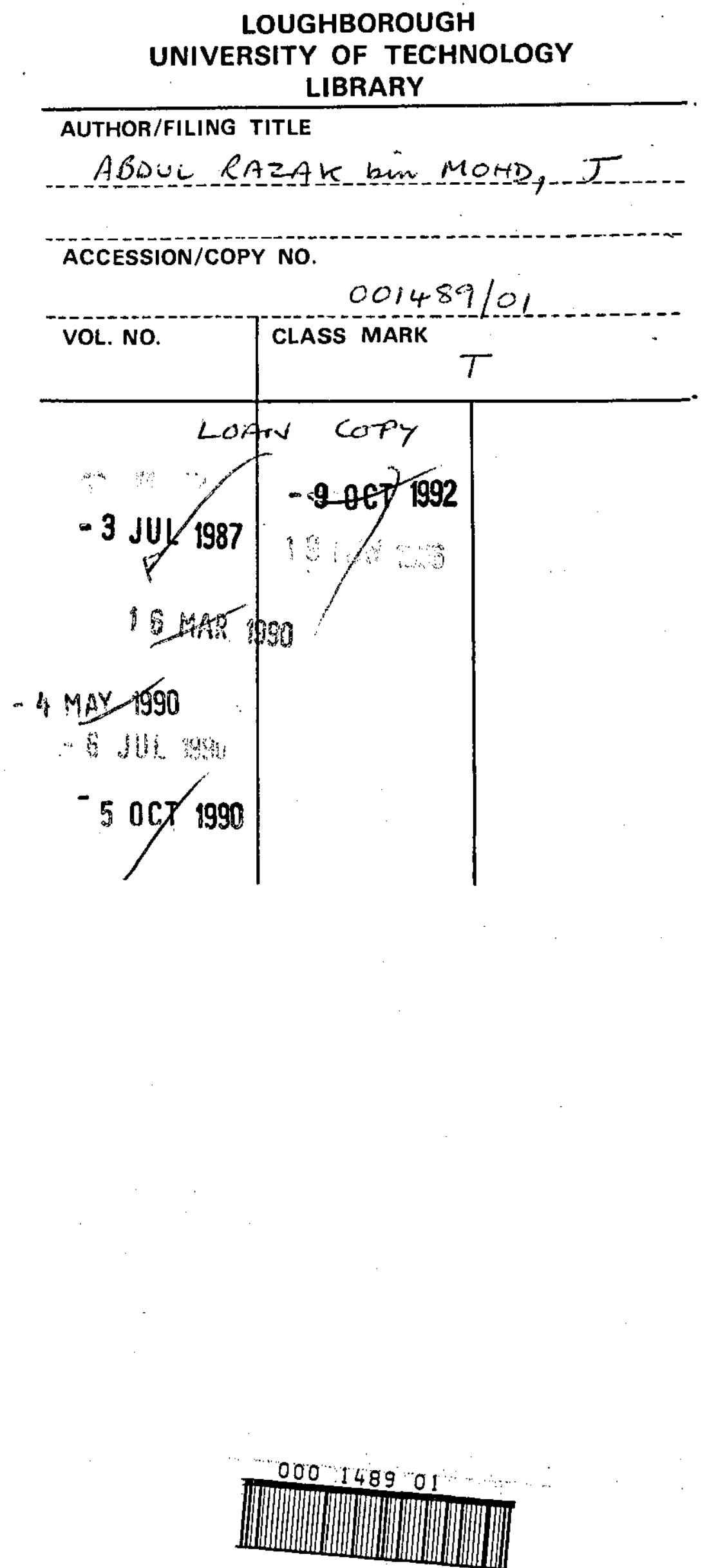



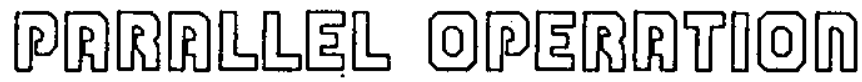 \\ op \\ BRODEFE RESTIFUERS

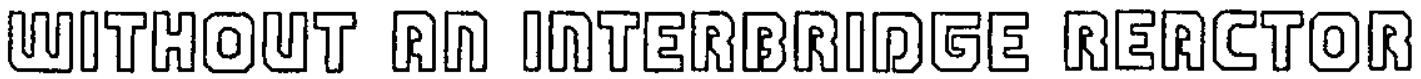

ABDUL RALAK BY $\stackrel{\text { by }}{M O H}$ MOD. IFM, BSC.

A Masten's thesis

Submitted in partial fulfilment of the requirements fon the awand of

Master of Science of the

Loughborough University of Mechnology

\section{January 1985}

Supervisors: Dr. J. K. Hall

Mr. J. G. Kettleborough Department of Electronic and Electrical Engineering

(C) by Abdul Razak, 1985 


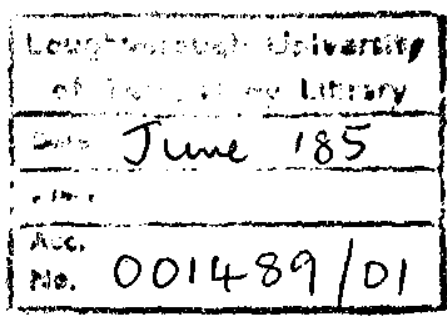




\section{SUMMARY}

The thesis reports an investigation into the operation and performance of a 12-pulse parallel bridge rectifier system supplied from a three winding-transformer and operating without an interbridge reactor. The work has been undertaken in liaison with GEC Transmission and Distribution Projects Ltd., Stafford. The conventional centretapped interbridge reactor on the dc side allows each bridge to operate independently of the other in its normal 6-pulse manner making the analysis fairly straightforward if the usual assumption of perfectly smooth direct current is made. The omission of the reactor can provide very substantial cost and space savings in high power installations. However, its absence leads to interaction between the bridge rectifiers, and this is of special interest here. Additionally, it has been found necessary to be able to predict accurately the transformer winding current waveforms. The system supplies a load of resistance, inductance and back emf which is typical of electrochemical plant for which this research has direct application.

A sophisticated tensor technique is employed for analysis and a mathematical model is formulated for implementation in a computer. Finite values for all resistances and inductances are used in the comprehensive analysis, with the tensors used to assemble the relevant mesh state variable equations which are dependent on the rectifier device conduction patterns. The selection of mesh currents as the state variables makes the model particularly suitable for use in large system studies with the aid of a computer.

The bridge supply, transformer winding and ac supply current waveforms are obtained, together with their harmonic components, and 
confirmed experimentally using an experimental laboratory system.

The now justified computer program is used to provide the operating characteristics, which incorporate the various modes of operation resulting from rectifiex interaction, and demonstrate the influence of circuit parameters. In addition the mean values of the dc output voltage, individual bridge and total output currents are predicted. The results are again verified experimentally.

It is concluded that the proposed analytical technique is successful for predicting the parallel rectifier system waveforms and performance, when operating without the conventional centre-tapped interbridge reactor on the dc side. The model also allows for its inclusion if required. 


\section{ACKNOWLEDGENEATSS}

First and foremost I wish to give all the Praise to Almighty God for giving me the strength and time to complete this research. With His Blessings may this work be beneficial for the whole of humanity.

I am deeply indebted to my supervisors Dr. J.K. Fall for his help and encouragement throughout this investigation and MI. J.G. Kettleborough for his valuable assistance in preparing the computer program. I would like to extend my appreciation to GEC Transmission and Distribution Projects Ltd., Stafford, who initially sponsored the work, for their helpful cooperation. My thanks are also due to the Loughborough University Computer Centre and the Department of Electronic and Electrical Engineering for granting the permission to use their facilities. Finally, to my wife and sons Abdul Hadi, Zaid, Mus'ab and Zubair for their presence which provided me with the encouragement to complete the work. 
SUMMARY

ACKNOWLEDGEMENTS

CONTENTS

LIST OF PRINCIPAL SYMBOLS

CHAPTER 1 INTRODUCTION

1.1 Background 1

1.2 Objectives 4

1.3 Method of approach 4

CHAPTER 2 OPERATING MODES

2.1 Description of circuit and its 6 representation

2.2 Mode 1: Discontinuous load current, discontinuous bridge current

2.3 Mode 2: Continuous load current,

discontinuous bridge currents, interbridge commutations.

2.4 Mode 3: Continuous load current,

continuous bridge currents, separated interphase commutations alternately in each bridge.

2.5 Mode 4: Continuous load current, 18 continuous bridge currents, part-coincidence interphase commutations between the bridges. 
2.6 Mode 5: Continuous load current,

continuous bridge currents,

complete coincidence of

bridge interphase commutations

with overlap interference

in each briage.

2.7 Summary of the operating modes

CHAPTER 3 SYSTEM MODELLING

3.1 Introduction

3.2 Assumptions 28

3.3 Method 29

3.4 The transformer-thyristor unit 29

3.5 Modeling the system 32

3.5.1 General

3.5.2 The primitive reference frame

3.5.3 The intermediate reference frame 37

3.5.4 The mesh reference frame 48

3.5.5 The master thyristor conduction matrix

3.5.6 Derivation of the transformation 50 $\operatorname{matrix} \mathrm{C}_{\mathrm{m}}^{1}$

CHAPTER 4 COMPUTER IMPLEMENTATION

4.1 Introduction. 53

4.2 The main program 54

4.3 Solution process 56

4.4 Trigger pulse for the thyristor circuits 61

4.5 Current discontinuity 64

$\begin{array}{lll}4.6 & \text { Interpolation } & 67\end{array}$ 
$\begin{array}{lll}4.7 & \text { Thyristor voltage } & 67\end{array}$

$\begin{array}{lll}4.8 & \text { Integration procedure } & 70\end{array}$

4.9 Optimum step length 71

$\begin{array}{ll}4.10 & \text { Input data }\end{array}$

CHAPTER 5 HARMONIC ANALYSIS AND MEAN QUANTITIES

5.1 Introduction $\quad 74$

5.2 Fourier Analysis $\quad 74$

5.3 Variation of the step length in the 77 estimation of the Fourier Coefficients

5.4 Computer estimation of the Fourier 80 Series for a known signal

5.5 Mean dc output quantities 80

5.6 Input data $\quad 84$

CHAPTER 6 RESULTS AND DISCUSSION

6.1 Introduction 85

6.2 The laboratory convertor system 86

6.3 Alternating current waveforms 94

6.4 Operating characteristics 118

CHAPTER 7 CONCLUSIONS

7.1 Summary of the work done 131

$\begin{array}{lll}7.2 & \text { Discussions } & 131\end{array}$

7.3 Suggestions for further work 133

REFERENCES $\quad 135$

$\begin{array}{lll}\text { APPENDIX I MEASUREMENT AND CALCULATION OF } & 138\end{array}$

TRANSFORMER PARAMETERS

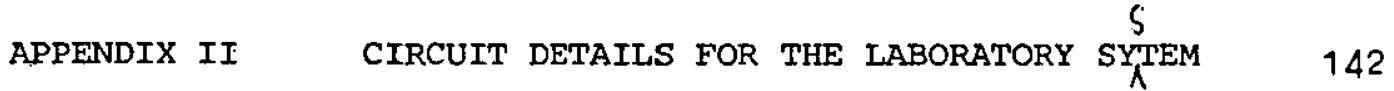


APPENDIX III COMPUTER PROGRAM LISTING FOR

PLOTTING WAVEFORMS

APPENDIX IV COMPUTER PROGRAM LISTING FOR HARMONIC ANALYSIS AND MEAN VALUES 


\section{LIST OF PRINCIPAL SYMBOLS}

$\alpha$

$\alpha_{1}$

$\alpha_{c_{2}}$

$\alpha_{c_{3}}$

$\alpha_{c_{4}}$

$\beta, \gamma, \delta, \psi, \lambda$

$\mu$

$\omega_{0}$

$\theta_{n}$

$\Delta_{t}, \Delta_{t_{r}}, \Delta_{t_{r-1}}$

c. $\cdot \vec{i}$

$c_{i}^{\cdot b}$

C. $\frac{i}{m}$

$C_{m}^{\cdot 1}$

$E_{R}, E_{Y}, E_{B}$

$\mathrm{E}_{\mathrm{b}}$

${ }^{\mathrm{E}} \mathrm{d}$

$E_{i}$

$\mathrm{E}_{\mathrm{m}}$

$I^{1}, I^{2}, I^{3}$... etc. - steady state current in the transformer windings

- firing delay angle*

- Ist critical firing delay angle*

- 2nd critical firing delay angle*

- 3rd critical firing delay angle*

- 4th critical firing delay angle*

- loop current cessation angles for Modes 1, 2, 3, 4 and 5 *

- interphase comutation period

- angular frequency

- phase angle of the $\mathrm{n}^{\text {th }}$ harmonic components

- step lengths

- the transformation matrix, intermediate reference frame to the primitive reference frame (ctran in the computer program)

- the transpose of $c .{ }_{i}^{b}$ (ctrant in the computer program)

- the transformation matrix, mesh reference frame to the intermediate reference frame (cb in the computer program)

- the transpose of $C \cdot \frac{i}{m}$ (cbt in the computer program)

- supply line voltages

- the impressed branch voltage vector

- back e.m.f.

- the referred impressed branch voltage vector

- the impressed mesh voltage vector 


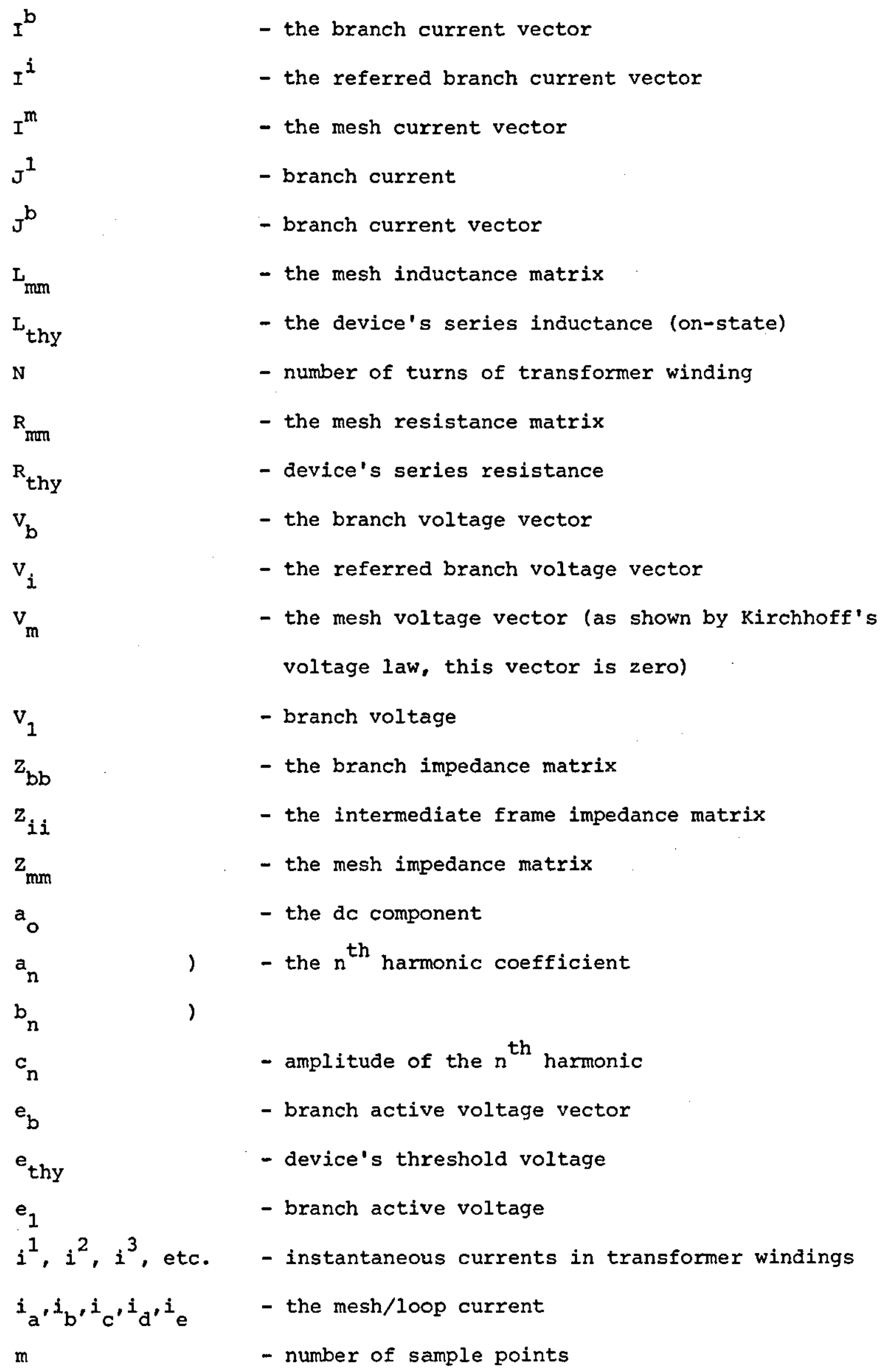$$
I^{b}
$$$$
I^{\dot{1}}
$$$$
\mathrm{I}^{\mathrm{m}}
$$$$
\mathrm{J}^{1}
$$$$
J^{b}
$$$$
\mathrm{L}_{\mathrm{mm}}
$$$$
\text { I thy }
$$$$
\text { N }
$$$$
\mathrm{R}_{\text {mon }}
$$$$
\mathrm{R}_{\text {thy }}
$$$$
\mathrm{v}_{\mathrm{b}}
$$$$
\mathrm{V}_{\mathbf{j}}
$$$$
\mathrm{v}_{\mathrm{m}}
$$$$
\mathrm{V}_{1}
$$$$
\mathrm{z}_{\mathrm{bb}}
$$$$
z_{i i}
$$$$
\mathrm{z}_{\mathrm{mm}}
$$$$
a_{0}
$$$$
a_{n}
$$$$
b_{n}
$$$$
c_{n}
$$$$
e_{b}
$$$$
\text { e thy }
$$$$
e_{1}
$$$$
i^{1}, i^{2}, i^{3} \text {, etc. }
$$$$
i_{a^{\prime}} i_{b^{\prime}} i_{c^{\prime}} i_{d^{\prime}} i_{e}
$$$$
\text { m }
$$

- the branch current vector

- the referred branch current vector

- the mesh current vector

- branch current

- branch current vector

- the mesh inductance matrix

- the device's series inductance (on-state)

- number of turns of transformer winding

- the mesh resistance matrix

- device's series resistance

- the branch voltage vector

- the referred branch voltage vector

- the mesh voltage vector (as shown by Kirchhoff's voltage law, this vector is zero)

- branch voltage

- the branch impedance matrix

- the intermediate frame impedance matrix

- the mesh impedance matrix

- the dc component

- the $\mathrm{n}^{\text {th }}$ harmonic coefficient

- amplitude of the $n^{\text {th }}$ harmonic

- branch active voltage vector

- device's threshold voltage

- branch active voltage

- instantaneous currents in transformer windings

- the mesh/loop current

- number of sample points 
$(x)$

n

$\mathrm{p}$

$t$

ta

$\mathrm{v}_{1}, \mathrm{v}_{2}, \mathrm{v}_{3}$, etc.

Y

$I_{\mathrm{d} 1}, I_{\mathrm{d} 2}$

$I_{d}$

$v_{d}$
- harmonic number

- operator

- time

- time for current discontinuity in a step

- instantaneous voltages in the transformer winding

- amplitude of a signal

- mean output bridge currents

- meen load current

- mean output voltage 


\subsection{Background}

The increasing size of electrochemical plant for manufacturing such products as chlorine, aluminium and copper, provides the need for ac-dc power convertors of up to the order of $30 \mathrm{MW}$, with direct currents exceeding $100 \mathrm{kA}$. To meet the demand, the manufacturers of rectifier convertors need to increase the current ratings of their equipment. At the same time they are required to limit the level of harmonic currents generated by these convertors. If six-pulse rectifiers are operated at very high currents, the lower order, more prominent, harmonics may easily exceed the levels acceptable to the Electricity Supply Authorities (ref: 1, 2). These conflicting requirements are met to a large extent if the pulse number of the convertor is increased, which improves the ac supply current waveform and also reduces the output voltage ripple.

Nowadays, thyristor convertors are popular for supplying direct current loads of ever increasing levels. This is because of their reliability, efficiency and controllability. These convertors use one of two basic rectifier circuit configurations:

(i) Two three-phase half wave rectifiers connected in parallel. through an interphase transformer (centre-tapped reactor) giving the double 3-phase star connection;

(ii) The three phase bridge.

For very high current levels, each device in the configuration is replaced by a group of parallel connected devices. Multiple pulse systems are realised by parallel connection of such circuits, frequently but not always using a further centre-tapped reactor.

It is apparent from the ac harmonics point of view that six- 
pulse rectifier operation is insufficient for the purpose outlined in the first paragraph. Therefore, an increase of the pulse number to either twelve, twentyfour or fortyeight is necessary for very high power rectification. This is achieved by providing two or more sixpulse circuits connected either in series or in parallel and supplied by suitably connected transformers. The transformer arrangement provides the appropriate phase displacement between the six-pulse circuits to give the desired output pulse number. For example, 12-pulse operation can be obtained by phase shifting two six-pulse circuits to give $30^{\circ}$ phase displacement. A 24-pulse operation can be obtained by using four 6-pulse circuits, $15^{\circ}$ phase shifted with respect to each other. Similarly, 48-pulse operation can be obtained by eight circuits, $7.5^{\circ}$ phase shifted with respect to each other. Such phase shifting substantially increases the transformer cost. Cref: 3$)$.

For medium and large power loads a 12-pulse system offers the best compromise, and is widely used in one of two configurations:

(i). Two double 3-phase star rectifiers with interphase transformer connected in parallel through an additional intergroup reactor;

(iil Two 3-phase bridge rectifiers in parallel.

The choice between these options is usually based on the required mechanical layout for satisfactory cooling of the many paralleled devices, and cost. Since parallel 3-phase bridges are coming more into use, configuration (ii) is considered in this work.

Two bridge circuits would normally be connected in parallel through an interbridge centre-tapped reactor (IPT) which sustains the instantaneous voltage difference in the output of the two bridges. Thus, this 
maintains the independent operation of both bridges as six-pulse rectifiers. At low and medium power levels an interbridge reactor is invariably included, but for very high power ratings this is a very large and costly component and is sometimes omitted on economical grounds. Then the operation of the bridges becomes vastly more complicated by their interaction, and there appears to be no evidence of a complete analysis having been made of such operation. When ayailable, such an analysis would give assistance to rectifier equipment and transformer designers, and contribute to reduce costs and increased reliability. This forms the justification for this work. Additionally, it is usual to adopt the simplifying assumptions of zero ac supply transformer resistance and infinite dc load inductance for the analysis of high power rectifier circults. This is not done here, finite values being allowed for.

When supplying two parallel bridges there are two basic transformex arxangements which will provide the required $30^{\circ}$ supply voltage displacement:

(i). Two separate three-phase transformers, one for each bridge, Faving the same primary winding connection with one secondary being star-connected, the other delta-connected;

(iil A single three-phase, three winding (per phase) transformex with the star-connected secondary supplying one bridge and the delta-connected tertiary the other.

The latter is the more complicated to consider analytically. In practice, the choice between the two is governed by economics.

The research has been carried out on the 12-pulse parallel bridge rectifier system without an interbridge reactor, and supplied by a 
three-phase, three-winding transformer. Both analytical and experimental work has been performed in depth. It furthers the work performed by Sood (ref: 2I who used an algebraic/Laplace transform method for the complete analysis of a single three-phase bridge. This approach proved too elaborate for adaptation to the more complex operation of two bridges in parallel without an IPT, and necessitated widening the scope of the work in order to find a simpler analytical technique. The nature of any method to be considered is that it must be orientated towards the digital computer since, inevitably with the complexity involved, computer assistance is essential.

\subsection{Objectives}

(i) To analyse fully the operation of the parallel bridge system with a 3-phase, 3-winding transformer supplying the two bridges;

(ii). To compute the waveforms of the currents supplying the bridges, in the transformer secondary and primary phases, and that drawn from the ac supply;

Ciil To predict the fiarmonic content of the aforementioned current waveforms;

(iv) To confirm by experimental measurement that the analysis is correct;

(vL To obtain the operating characteristics of the system with varying circuit parameters

\subsection{Method of Approach}

In the first method of analysis considered, the three-winding transformer was modelled using its three-branch equivalent circuit per phase (ref: 4) as is usual for the calculation of voltage regulation 
under sinusoidal conditions. The secondary and tertiary branches supplied the two bridges. For each of the possible thyristor conduction patterns, a set of differential equations were derived from the complete equivalent circuit. These were then formulated into a state variable form and solved on the computer using the Funge-Kutta fourth order system.

This analysis gave results which were reasonably close to those measured experimentally (ref: 5L, but was discarded for two reasons: (i) Dissatisfaction regarding the accuracy of the three-winding transformer equivalent circuit in allowing for all mutual inductances between windings within each phase and between phases;

(iil. On deeper reflection, the need to include an additional loop current, to make the analysis topologically correct; this was found difficult to achieye as its path included many unidirectional switching elements.

The second approach considered, and uged is basically similar to that above in that again loop currents are obtained from the relevant mesh state variable equations, which are dependent on the thyristor conduction patterns. However, the difference lies in the avoidance of a three-winding transformer equivalent circuit by using the actual circuit configuration and parameters with tensor analysis. The assembly of the state variable equations is performed automatically by the computer, the tensors being employed basically as transformation matrices to relate the disconnected (primitive) branches of the system to the connected system.

After the bridge supply, transformer winding and total supply currents are derived and confirmed experimentally, the system operating characteristics are computed and similarly confirmed experimentally. 
CHAPTER 2 OPERATING MODES

\subsection{Description of the Circuit and its Representation}

Fig. 2.1 shows the schematic diagram of the 12-pulse parallel rectifier bridge system under consideration. The two thyristor bridge rectifiers are connected in parallel without an interbridge reactor (IPT). Each bridge alone gives a six-pulse output and by supplying them with voltages which are $30^{\circ}$ displaced, a 12-pulse output is obtained from the parallel arrangement. A three winding transformer is used, and experimentally this comprises three separate three-winding transformers, one for each phase. For equal output voltages, the number of turns per!phase in the star connected secondary winding are $1 / \sqrt{3}$ times those on the delta tertiary winding. A delta connected winding is used on the primary side.

The equivalent circuit arrangement used for the analysis is depicted in fig. 2.2. Each of the transformer windings is represented by a designated branch impedance, $\mathrm{Z}$ which comprises a branch resistance $R$ and a branch self-inductance $L$. Included also are the mutual inductances between the rindings in each phase and between phases (e.g. $\mathrm{L}_{14}$ and $\mathrm{I}_{12}$ respectively). As the transformer arrangement used for experiment has separate phases, the mutual inductances between the phases become zero wien the circuit data is included. This will be clearly indicated in chapter 3. For each of the thyristors, a voltage source and an impedance are included in series to represent the yoltage drop during conduction. When blocking, the thyristors are represented by an infinite impedance. A typical electrochemical load of resistance, inductance and back e.m.f. is used. 

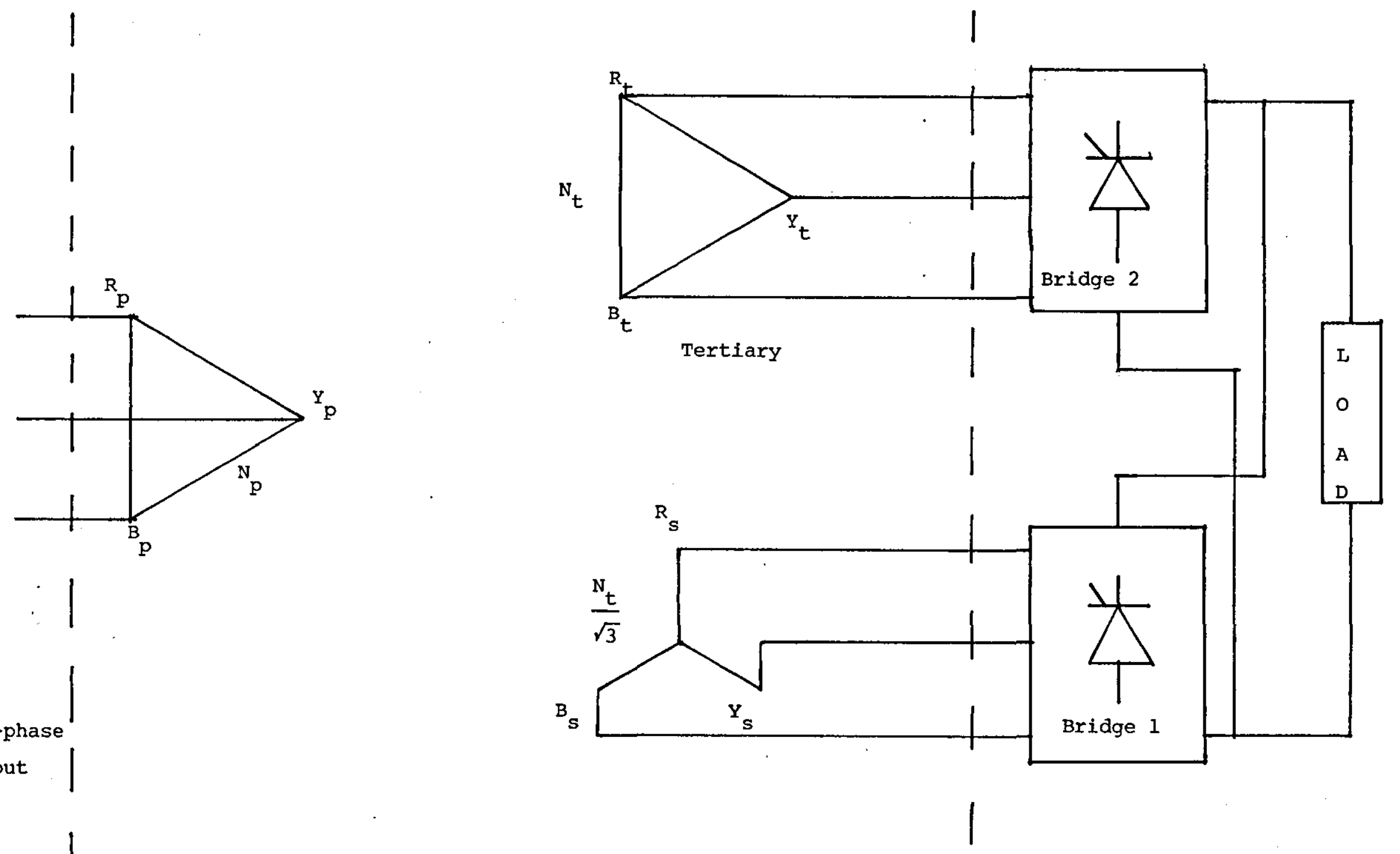

Primary

Secondary

Three-winding Rectifler Transformer

Two Three-phase Bridges in Parallel

FIG. 2.1 Schematic Diagram of the Twelve-Pulse System. 


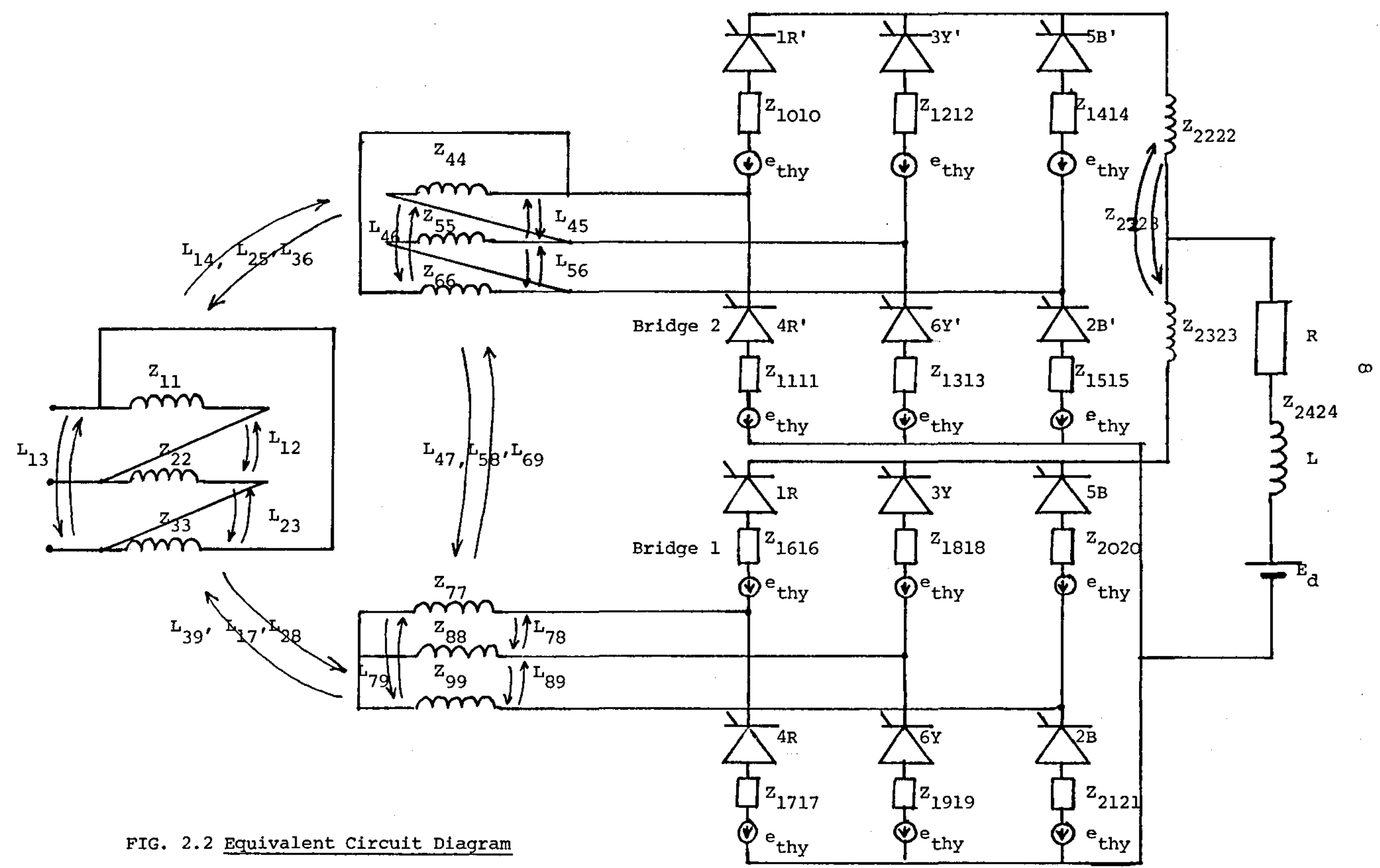


With this parallel bridge system without IPT various operating modes are possible, these being governed by the circuit and operating parameters. Sood (ref: 2) defined three modes of operation and these can be extended to five or more modes. Only four operating modes are realistic in practical terms but five modes will be presented for completeness.

When defining the operating modes of convertors it is conventional to consider the situation with continuous load current flow and small commutation angles which do not overlap as Mode 1. The further modes occur at higher current loadings where the operation changes, load current flow always being continuous (ref: 5 ). Here it is more logical to define Mode 1 as that which is the simplest analytically, where there is discontinuous load current flow with only one current loop through the load during conduction. As the mode number rises, the operation and analysis become increasingly complex, with interaction between the bxidges showing in theix simultaneous conduction during interbridge comnutations and overlapping of the individual bridge interphase commutations. The individual operating modes will now be considered.

\subsection{Mode 1: Discontinuous Load Current Discontinuous Bridge Currents}

Fig. 2.3 shows the conduction patterns for the five operating modes during about $150^{\circ}$ of the operating cycle. In Mode 1 , the load, bridge and thyristor current flows in short pulses having a conduction interval $\leqslant 30^{\circ}$. The bridges conduct alternately into the load, there being one load current loop in operation at a time, say $i_{a}$ in fig. 2.4. 


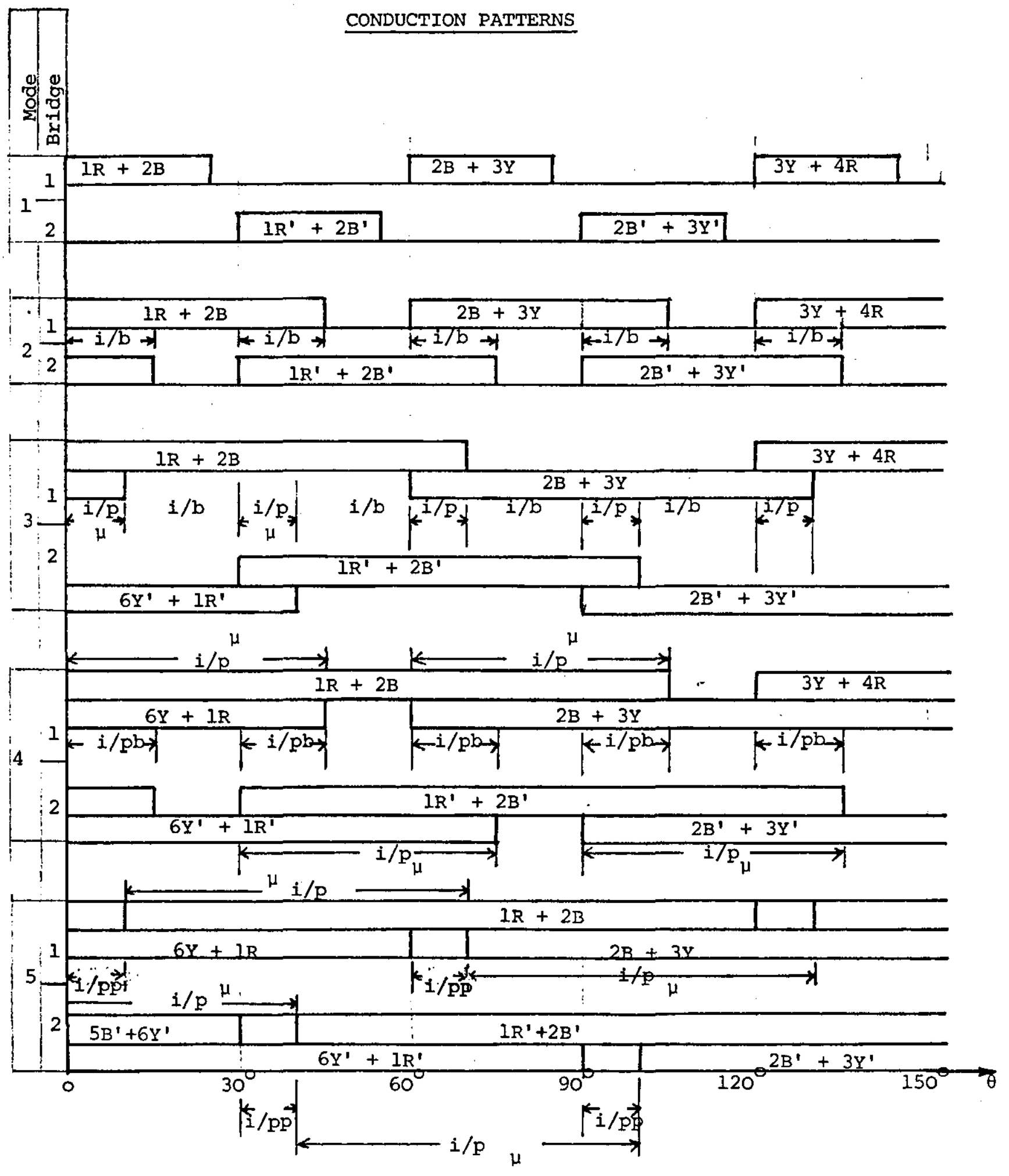

FIG. 2.3 Conduction Patterns for the Various Operating Modes

Bridge 2 conduction lags Bridge 1 conduction by $30^{\circ}$.

Interbridge commutations are shown $i / b$

Interphase commutations are shown $i / p$

overlapping interphase commutations between bridges are shown $i / p b$

Interphase commutation overlap interference within each bridge is shown $i / p p$ 
The type of waveforms to be expected are shown in fig. 2.5, with a conduction interval $(\beta-\alpha)$. Throughout, the firing delay angle $\alpha$ is measured from the zero voltage point of the appropriate bridge supply line voltage. This is a more convenient reference than the usual voltage crossover point which is $75^{\circ}$ later in a 12-pulse system, as shown in the figure.

\subsection{Mode 2: Continuous Load Current,}

Discontinuous Bridge Currents, Interbridge Commutations.

The second operating mode occurs when the firing delay angle is reduced such that the bridge (and thyxistor) conduction interval rises to greater than $30^{\circ}$ but less than $60^{\circ}$, the load current being continuous. The point where $(\beta-\alpha)=30^{\circ}$ is referred to as the first critical firing delay angle $\alpha_{\mathrm{Cl}}$, being where the modes change from 1 to 2 . During Mode 2 operation, one bridge is fired before the alternate bridge has ceased to conduct. This starts the transfer of load current from one bridge to the other, i.e., an interbridge commutation as shown in fig, 2.3. Referring to the circuit in fig. 2.6, thyristors $I R$ and $6 Y$ (say) are conducting the falling outgoing current $i_{a}$ and thyristors $I R^{\prime}$ and $6 Y^{\prime}$ carry the rising incoming current $i_{b}$. When $i_{a}$ falls to zero, the interbridge commutation is completed, $i_{b}$ then flowing singly for the rest of the conducting period until the next thyristor pair in sequence (IR and $2 B$ ) is fired. This process is repeated for the whole cycle of operation.

It should be noted that duxing a bridge 1 to bridge 2 interbridge commutation, devices in the same phases are taking over conduction, e.g. $I R$ and $6 Y$ transfer conduction to $1 R^{\prime}$ and $6 Y^{\prime}$. During a bridge 2 


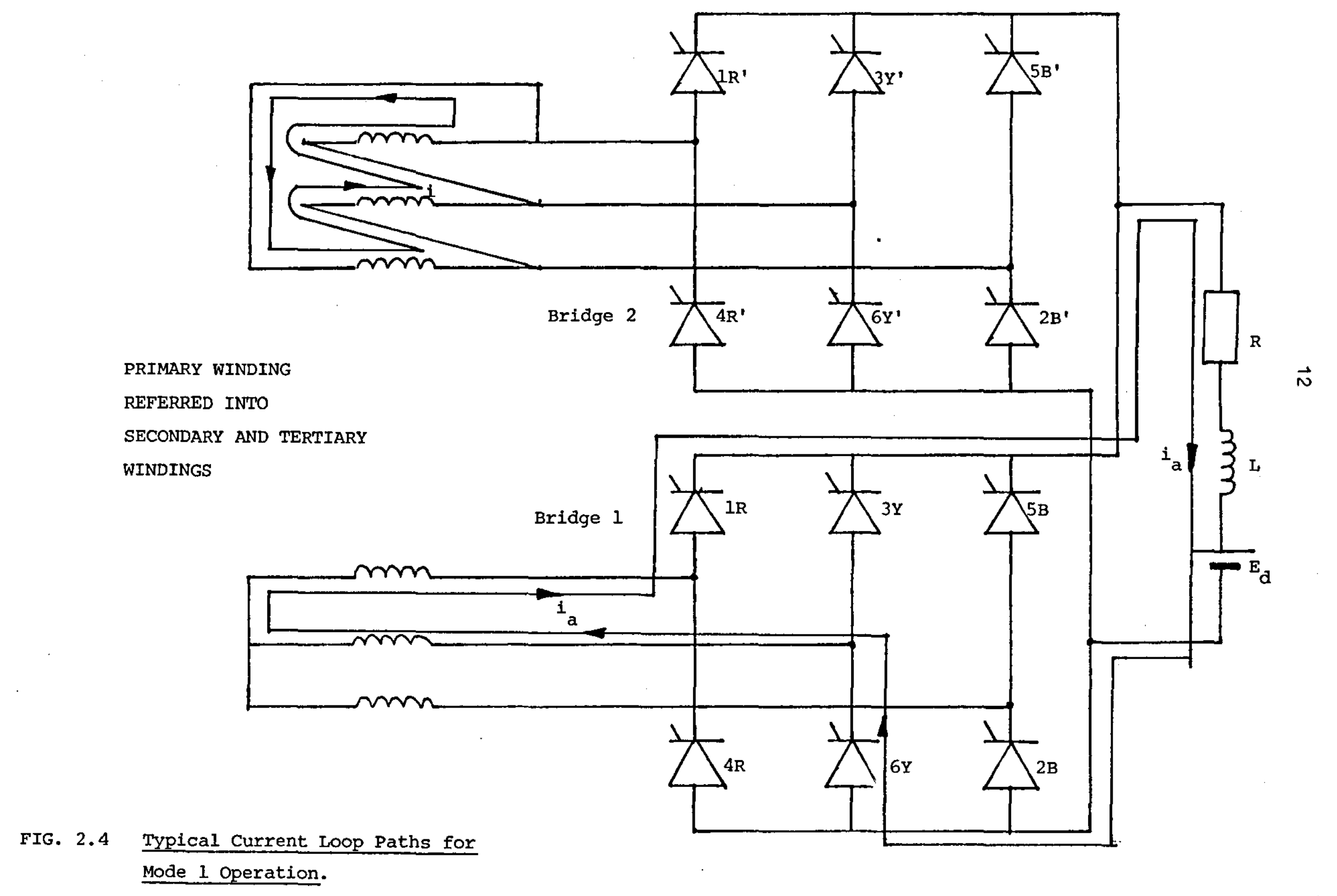




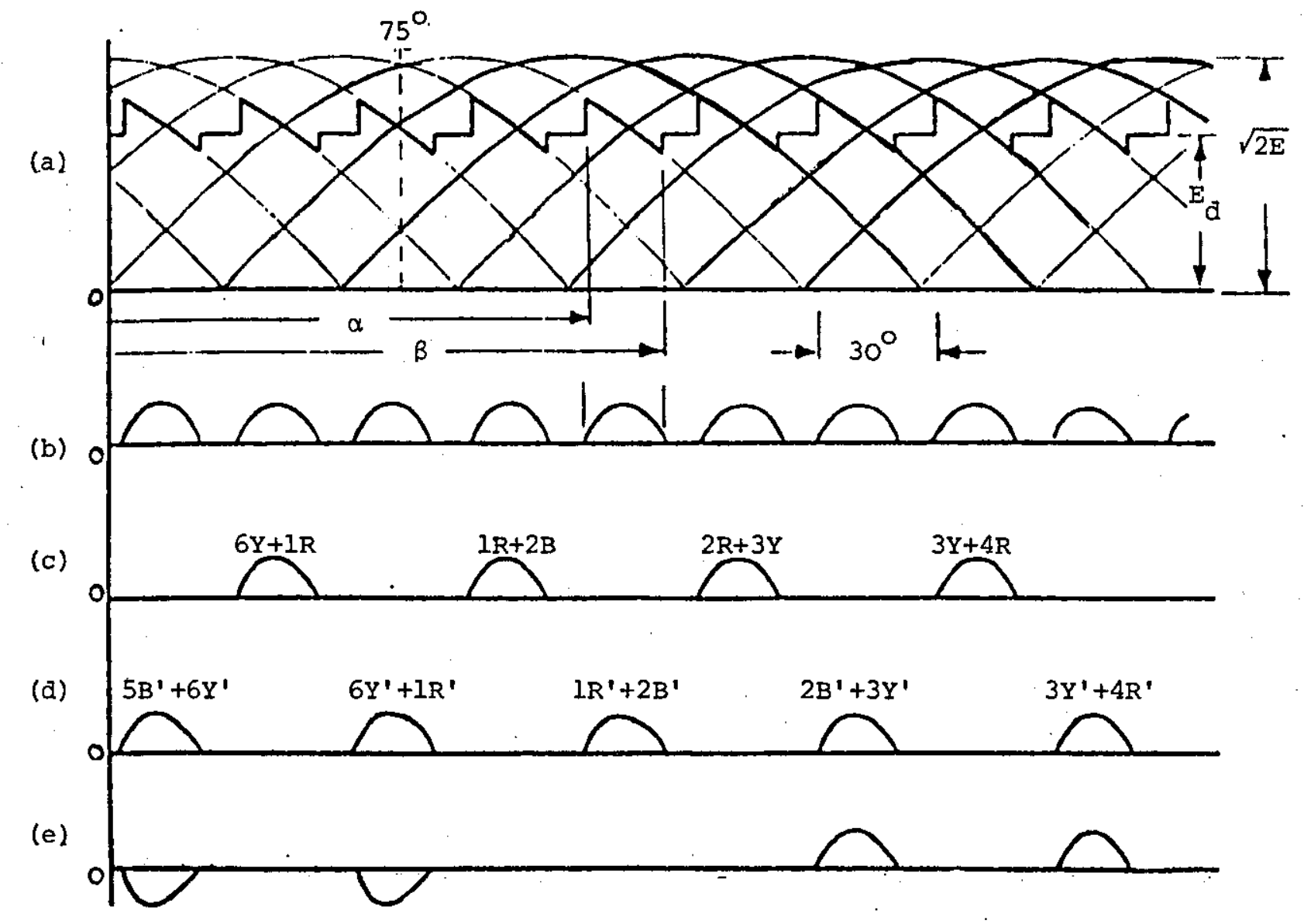

FIG. 2.5 Mode 1 Waveforms

Key: (a) dc output voltage;

(b) load current;

(c) bridge 1 current;

(d) bridge 2 current;

(e) bridge 2 supply line current $I_{Y}^{\prime}$

$\beta=$ angle of cessation current flow (measured from voltage zero) 


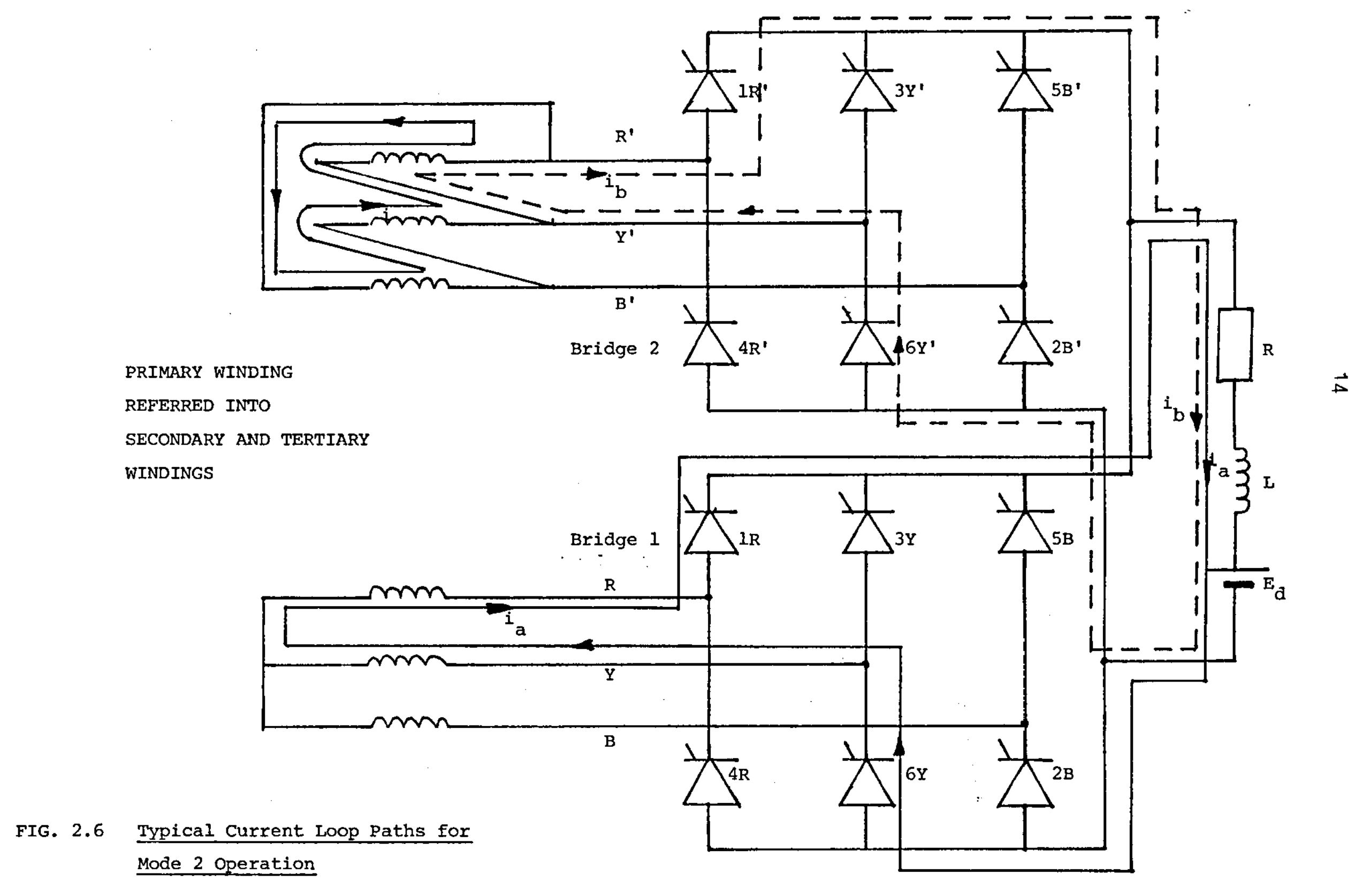


to bridge 1 commutation, the third phase is involved, e.g. $1 R^{\prime}$ and $6 Y^{\prime}$ transfer current to $\mathrm{R}$ and $2 \mathrm{~B}$. Thus, the transformer current paths diffex between the two interbridge commutations.

Fig. 2.7 shows the typical waveforms. The load current is made up of two alternate parts as follows:

(i) An interbridge commutation interval $(\gamma-\alpha)$ where the load current is the sum of the two bridge currents, one rising and the other falling;

(ii) A single conduction interval where only one bridge conducts. Each thyristor conducts with two pulses per cycle as in Mode 1.

\subsection{Mode 3: 'Continuous Load Current, Continuous Bridge Currents, Separated Interphase Commutations Alternately in Each Bridge}

If firing delay angle in Mode 2 is further reduced, the interbridge commutation interval $(\gamma-\alpha)$ will increase and the single conduction interval becomes shorter until a point is reached where the whole of the $30^{\circ}$ pulse width is now taken up by the interbridge commutation process. The firing delay angle then is $\alpha_{\mathrm{C} 2}$, the second critical firing delay angle. The operation is now about to change to the third operating mode, with conduction pattern as in fig. 2.3. In the Mode 3 operation, with the firing delay angle less than ${ }^{\alpha}{ }_{\mathrm{C} 2}$, the sequence of events is as follows:

(i) Let an interbridge commutation be occurring between the two bridges, one incoming, the other outgoing; load loop currents $i_{b}$ and $i_{a}$ respectively flowing in bridges 2 (incoming) and 1 (outgoing) as in fig. 2.8 . 
(a)

(b)

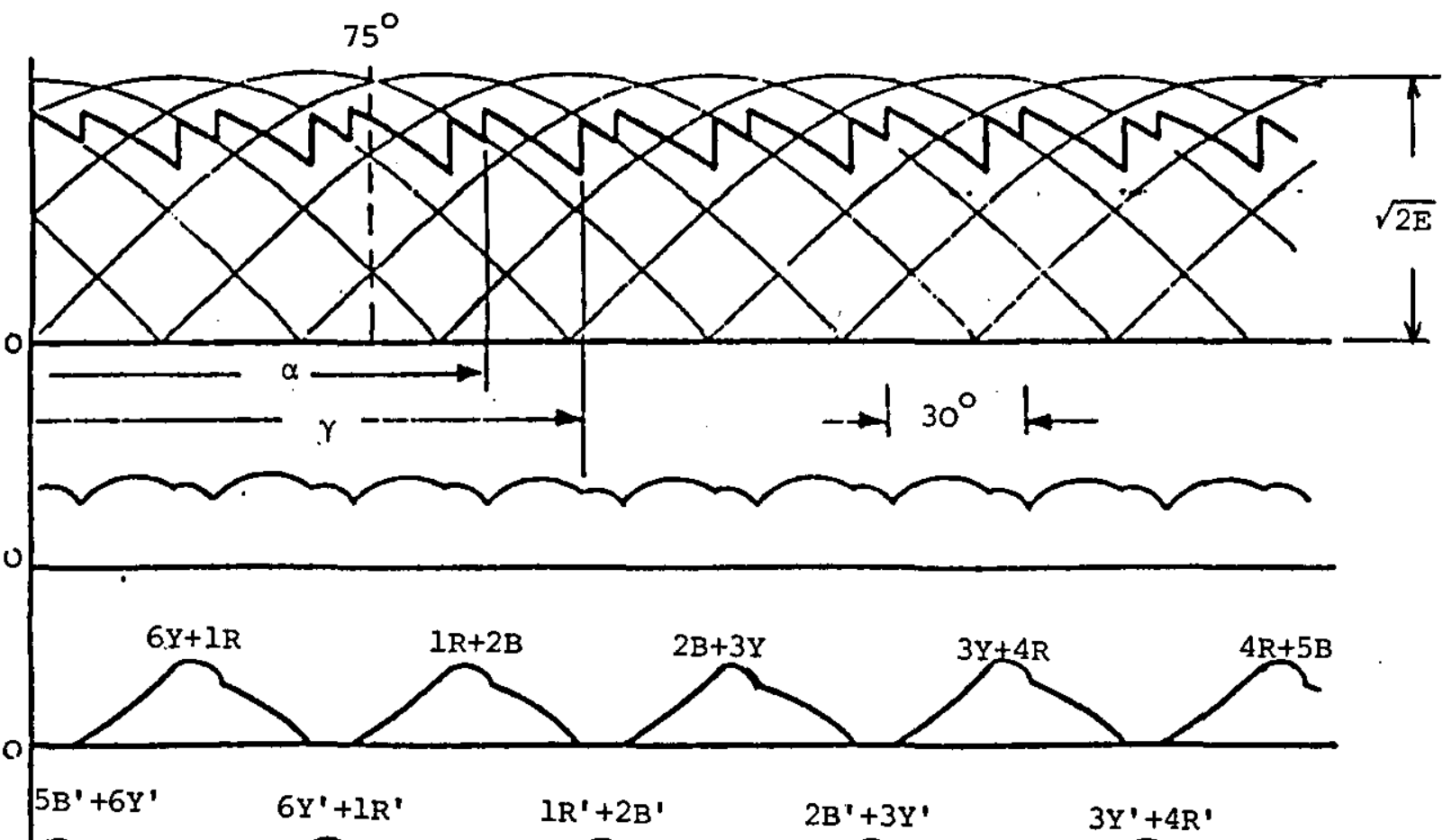

(d)

(c)

(d)

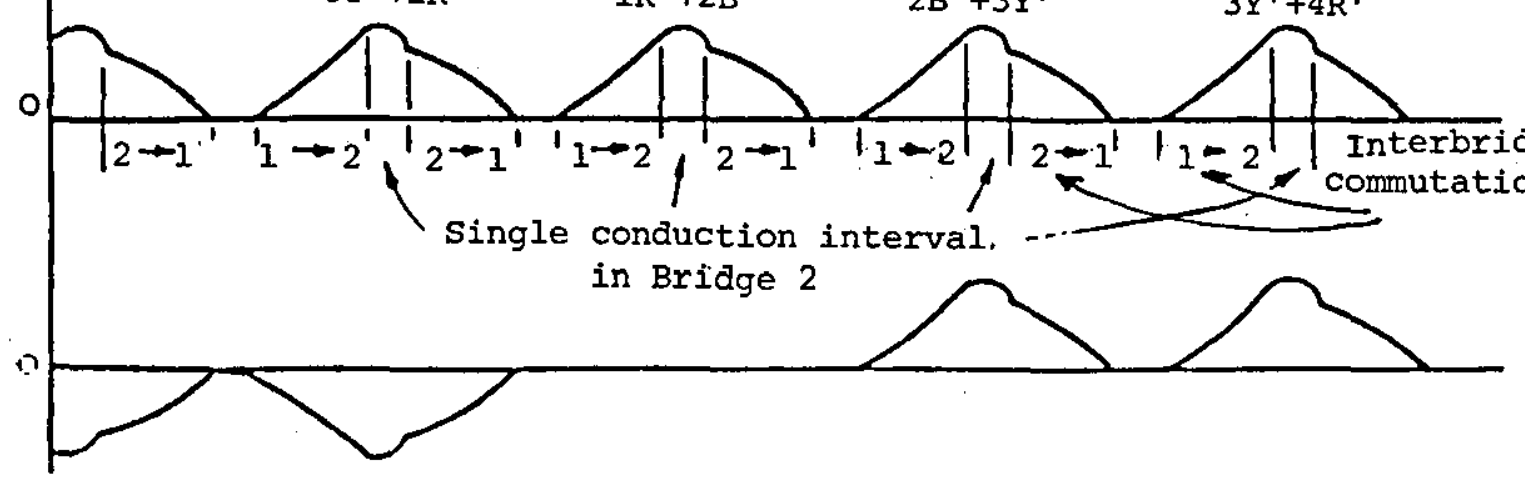

FIG. 2.7 Mode 2 Waveforms

Key: (a) dc output voltage;

(b) load current;

(c) bridge 1 current;

(d) bridge 2 current;

(e) bridge 2 supply line current $I_{y}^{\prime}$

$\gamma=$ angle at completion of interbridge commutation. 


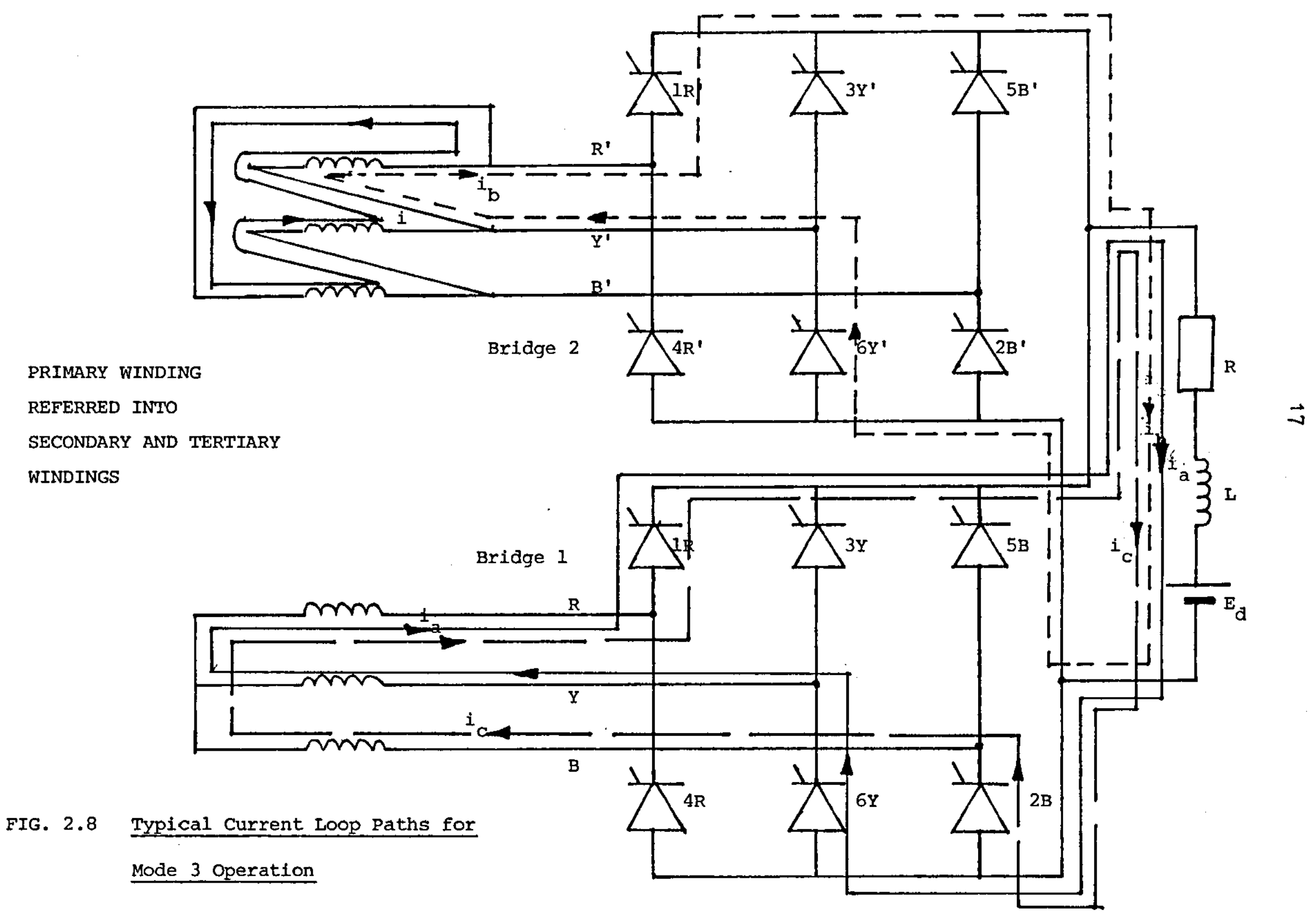


(ii) Before the interbridge commutation is completed, the next thyristor in sequence $2 B$ in the outgoing bridge is fired and an interphase commutation takes place in that bridge, $6 \mathrm{Y}$ current transferring to $2 \mathrm{~B}$ with an additional load loop current $i_{c}$ flowing;

(iiil On completion of the interphase commutation, $i_{a}$ has fallen to zero leaving an interbridge commutation with the bridge roles reversed, i.e. the previous incoming bridge now being the outgoing bridge and vice versa, currents $i_{b}$ and $i_{c}$ still flowing. The interphase commutation angle $\mu<30^{\circ}$. The conduction period for an individual thyristor is between $120^{\circ}$ and $150^{\circ}$ per cycle, the two pulses of Mode 2 having merged. Both the load current and the bridge currents are continuous with both bridges conducting permanently and interphase commutations occurring alternately in the bridges. Fig. 2.9 shows typical waveforms. Since either interphase or interbridge commutations are occurring at any instant, the dc output voltage at no time follows the ac supply but is always some value between the appropriate phase voltages depending on the type of commutation.

\subsection{Mode $4: \cdots$ Continuous Load Current, Continuous Bridge}

\section{"Currents; Part-Coincident Interphase Commutations}

\section{Between the Bridges.}

If the firing delay angle in Mode 3 is reduced to a third critical value $\alpha_{C 3}$ ' the bridges will operate with interphase commutations taking the whole of the $30^{\circ}$ pulse width. At the instant the interphase commutation in the bridge ceases, the other bridge starts its interphase 
(a)

(b)

(c)

0

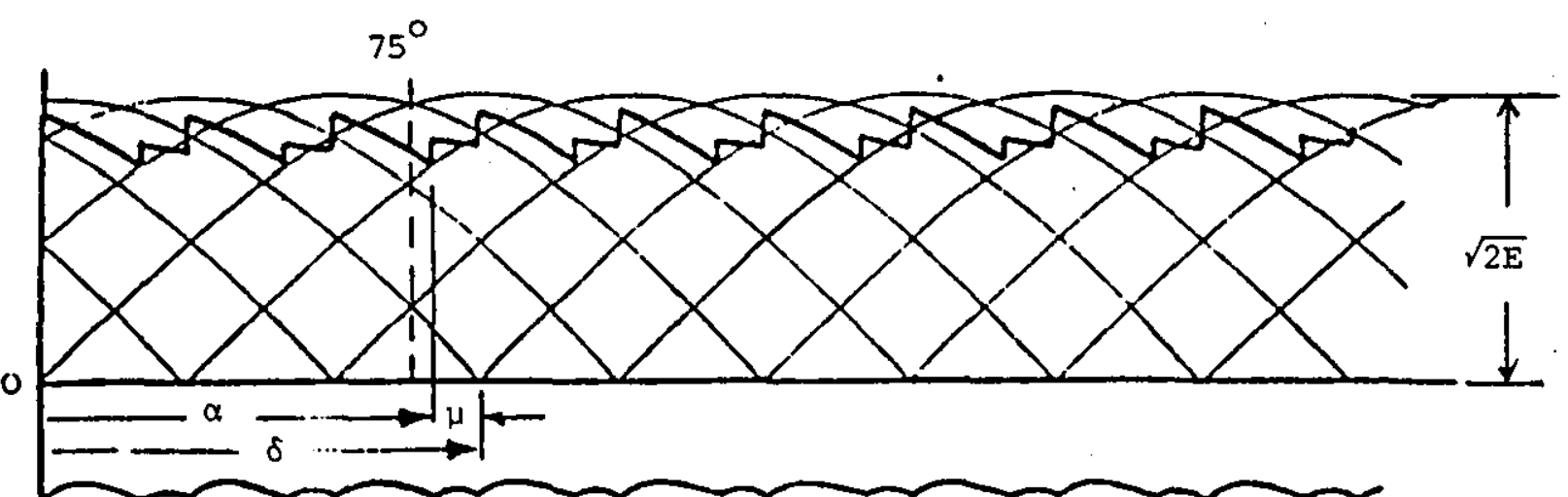

(d)

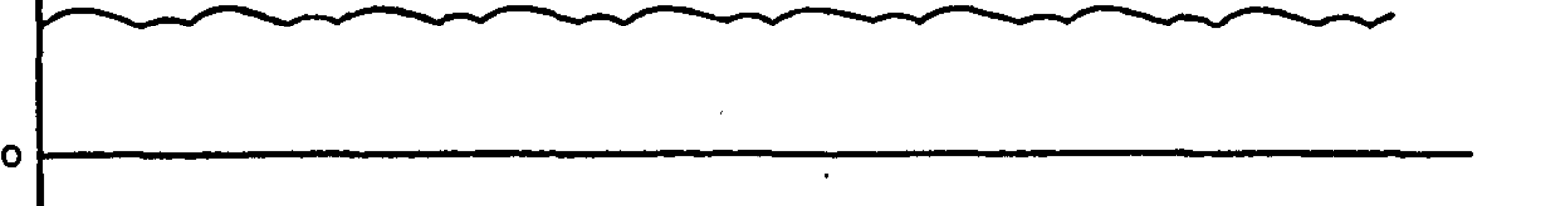

(e)

FIG. 2.9 Mode 3 waveforms

Key: (a) dc output voltage;

(b) load current;

(c) bridge 1 current;

(d) bridge 2 current;

(e) bridge 2 supply line current $I_{y}^{\prime}$

$\delta=$ angle of completion of interphase commutation within a bridge

$\mu=$ interphase commutation angle 
commutation. By making the firing delay angle less than $\alpha_{c 3}$, the system operates in Mode 4 with the bridge interphase commutations $\mu>30^{\circ}$ and therefore overlapping to some degree (Fig. 2.3). Referring to Fig. 2.10, an additional loop current $i_{d}$ is now present, and the operation is as follows:

(i) First let an interphase commutation be occurring in bridge 1 between phases $Y$ and $B$ with thyristors $2 R$ and $2 B$ carrying the incoming current, $i_{C}$ and $I R$ and $6 Y$ carrying the outgoing current, ${ }^{i_{a}}$, bridge current being commutated from $6 \mathrm{Y}$ to $2 \mathrm{~B}$. Bridge 2 carries a single current, $i_{b}$;

(ii) Before the briage 1 interphase commutation is completed, the next thyristor in sequence $2 \mathrm{~B}^{\prime}$ in bridge 2 is fired and a new interphase commutation occurs there with loop current $i_{d}$ rising, the bridge 2 current being commutated from $6 \mathrm{Y}^{\prime}$ to $2 \mathrm{~B}^{\prime}$;

(iii) on completion of the interphase commutation in bridge 1, when I falls to zero, the interphase commutation remains in bridge 2 with currents $i_{d}$ incoming and $i_{b}$ outgoing. The single loop current $i_{c}$ remains in bridge 1 .

Typical waveforms are shown in Fig. 2.11. For each bridge the sequence is:

a) Single loop current;

b) Interphase commutation starts, overlapping that ending in the other bridge;

c) Interphase commutation only in this bridge;

d) Interphase commutation overlapping the one starting in the other bridge and then ending.

The commutations in the bridges overlap by an angle $(\psi-\alpha)$ (Fig. 2.11) 


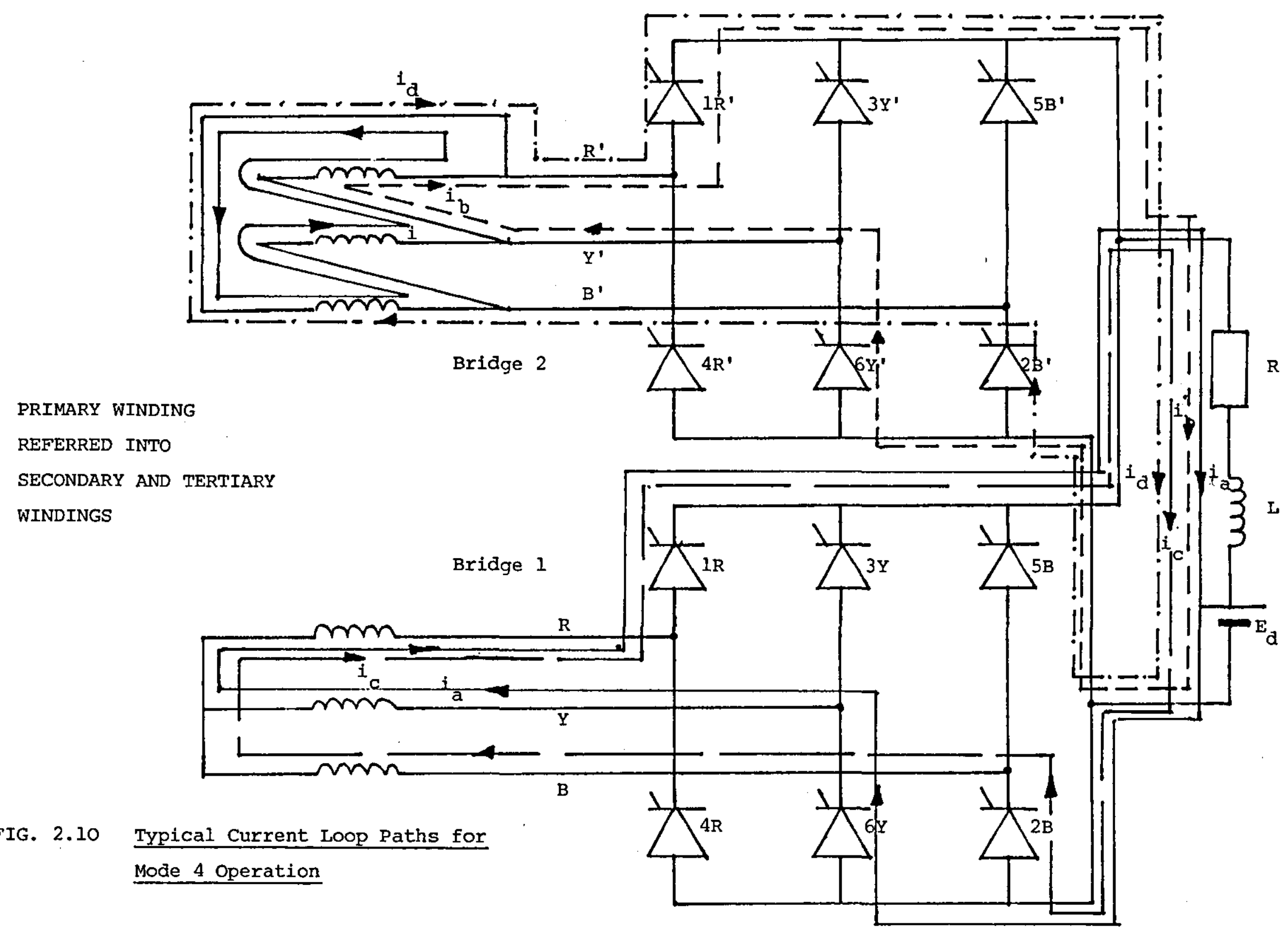




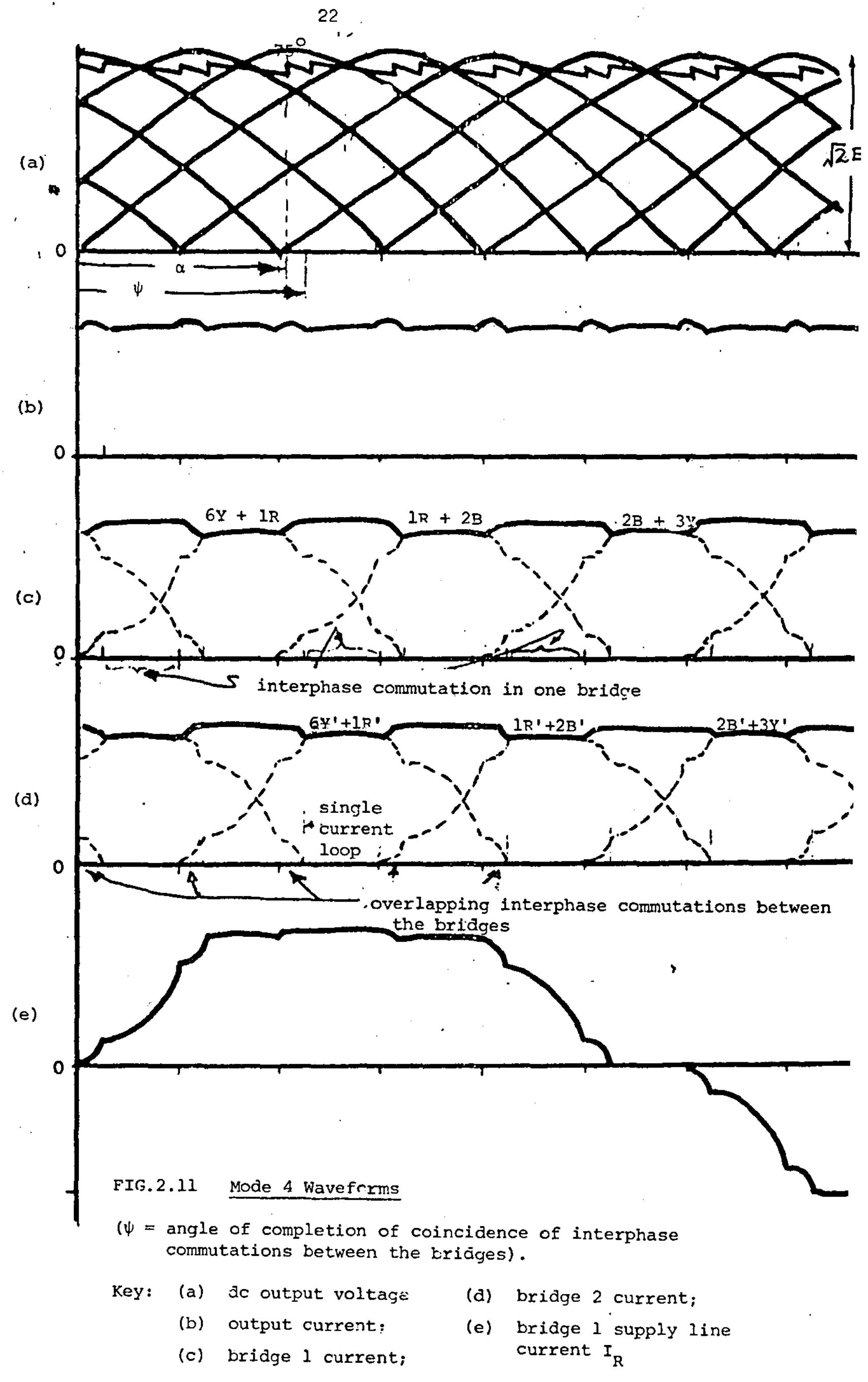


which is equal to the bridge interphase commutation angle $\mu$ less $30^{\circ}$. The sequence is repeated for the whole cycle, with each thyristor conducting for between $150^{\circ}$ and $180^{\circ}$.

\subsection{Mode 5: Continuous Load Current, Continuous Bridge Currents,}

Complete Coincidence of Bridge Interphase Commutations with Overlap Interference in Each Bridge.

A further reduction of firing delay angle to a fourth critical value $\alpha_{C 4}$ can bring a further change of operating mode. Then the bridge interphase commutation angles will be $60^{\circ}$, both bridges being permanently in a state of interphase commutation with three thyristors always conducting.

A further reduction of $\alpha$ (or increase of load current) produces a tendency for a rise in commutation overlap angle to $>60^{\circ}$, with a fourth thyristor gated in the bridge, this latter necessarily being associated with the same phase as that thyristor carrying the outgoing commutation current ( $Y$ in Fig. 2.12). As long as this outgoing current $\left(i_{a}\right)$ is flowing the newly gated thyristor (3Y) cannot conduct since its anode will be negative with respect to its cathode. This gives a period of interphase commutation overlap interference until the outgoing thyristor current $\left(i_{a}\right)$ falls to zero, and the (still gated) new thyristor (3Y) commences to conduct ( $i_{\mathrm{e}}$ ) giving the start of a fresh interphase commutation. Thus, all intexphase commutation angles are constrained to be $60^{\circ}$ permanently in Mode 5. Commutation overlap interference in single bridges is well known (Ref. 6). Fig. 2.3 again gives the conduction pattern and Fig. 2.13 the projected waveforms. It can be seen, for example, that the thyristor $3 y$ current rise commences 


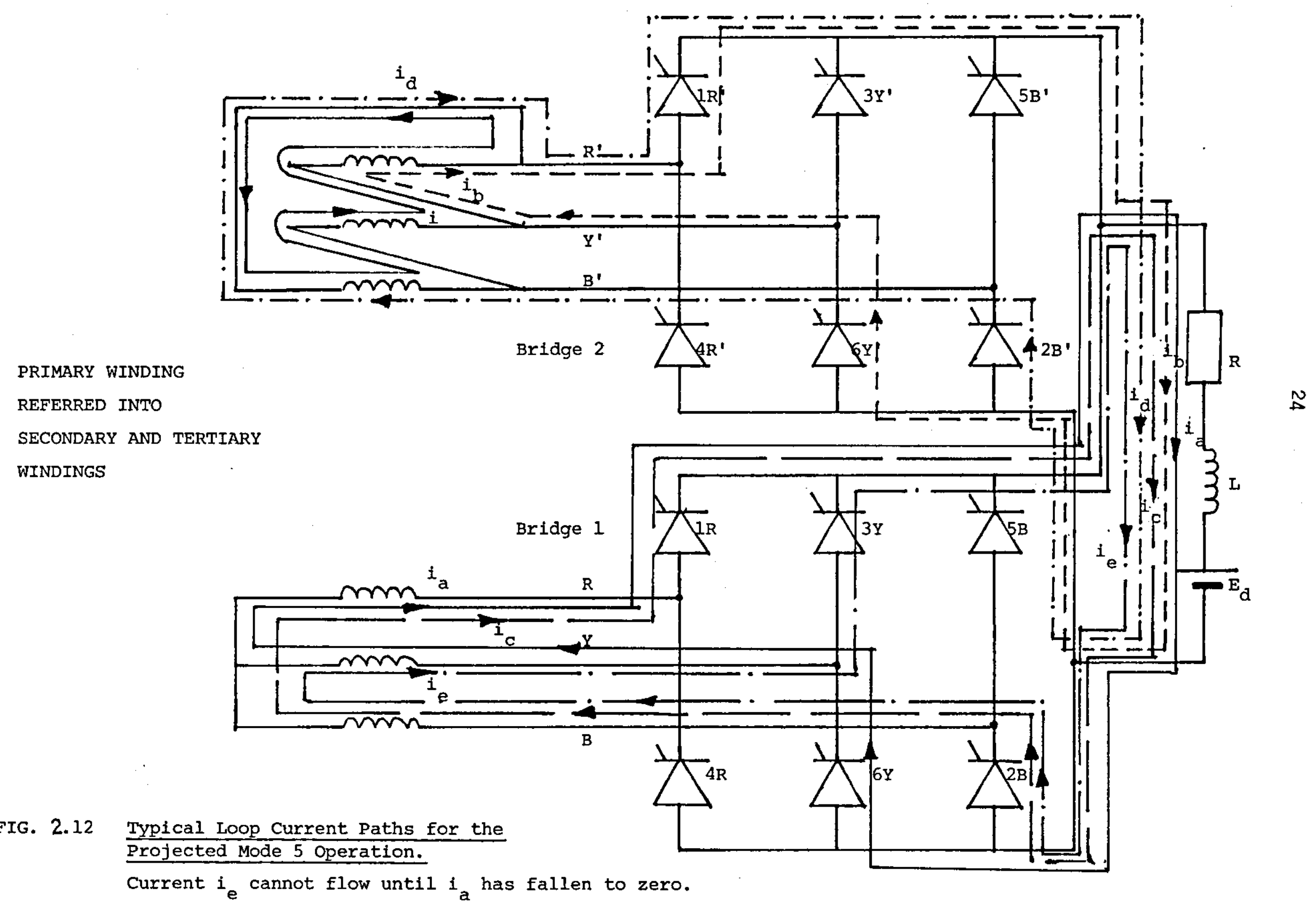


(a)

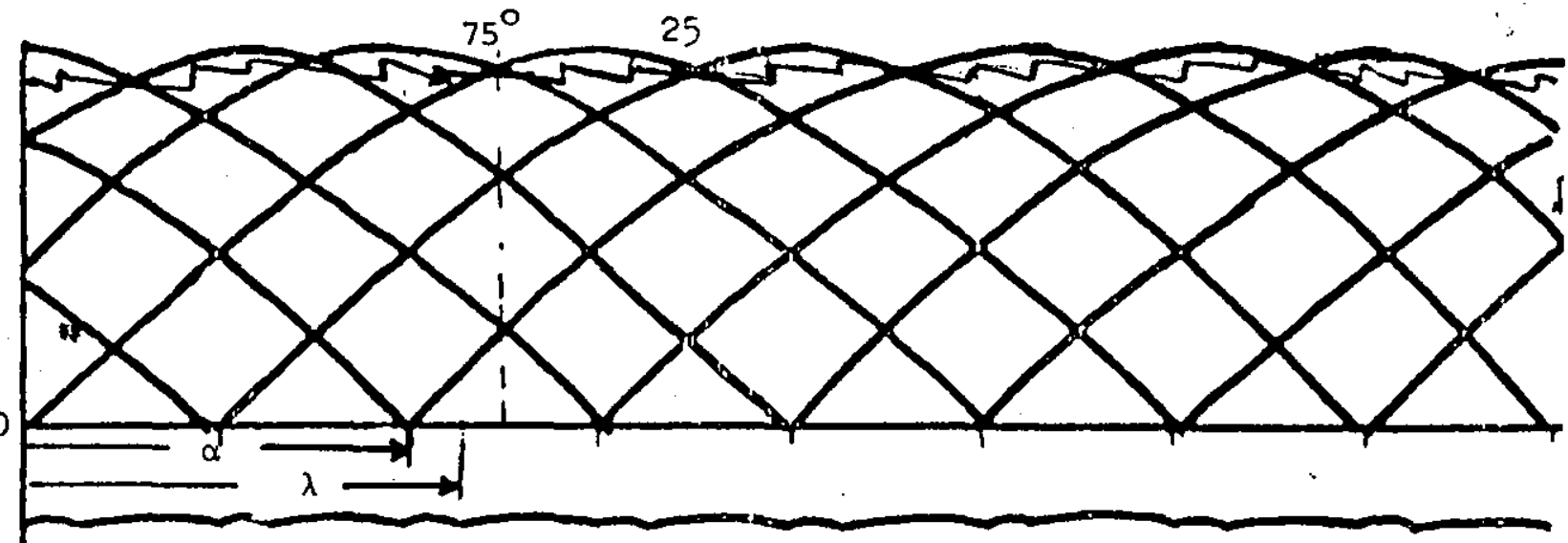

(b)

(c)

(a)

(e)

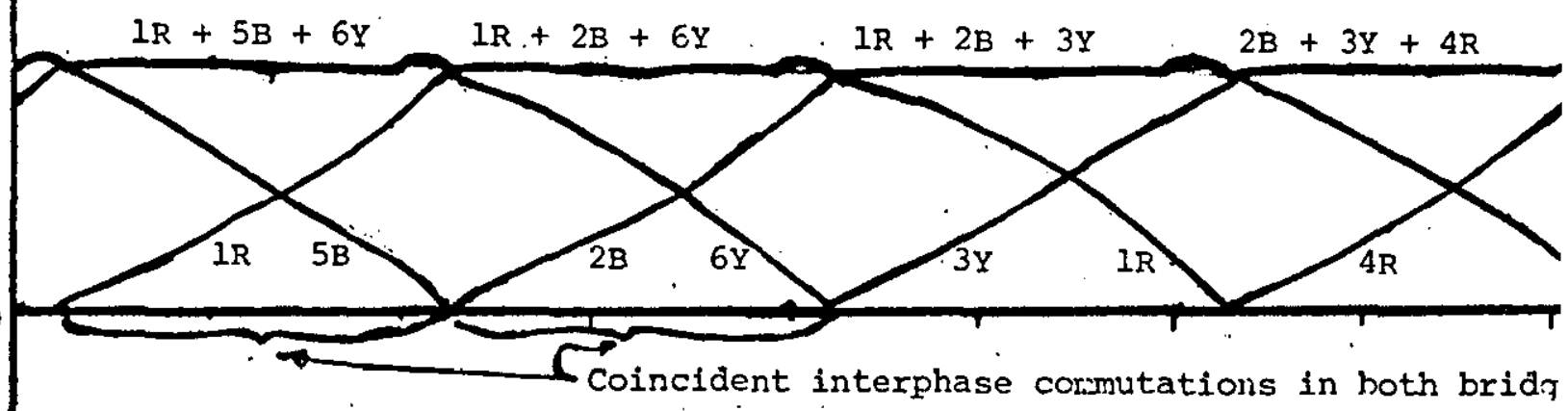

a)
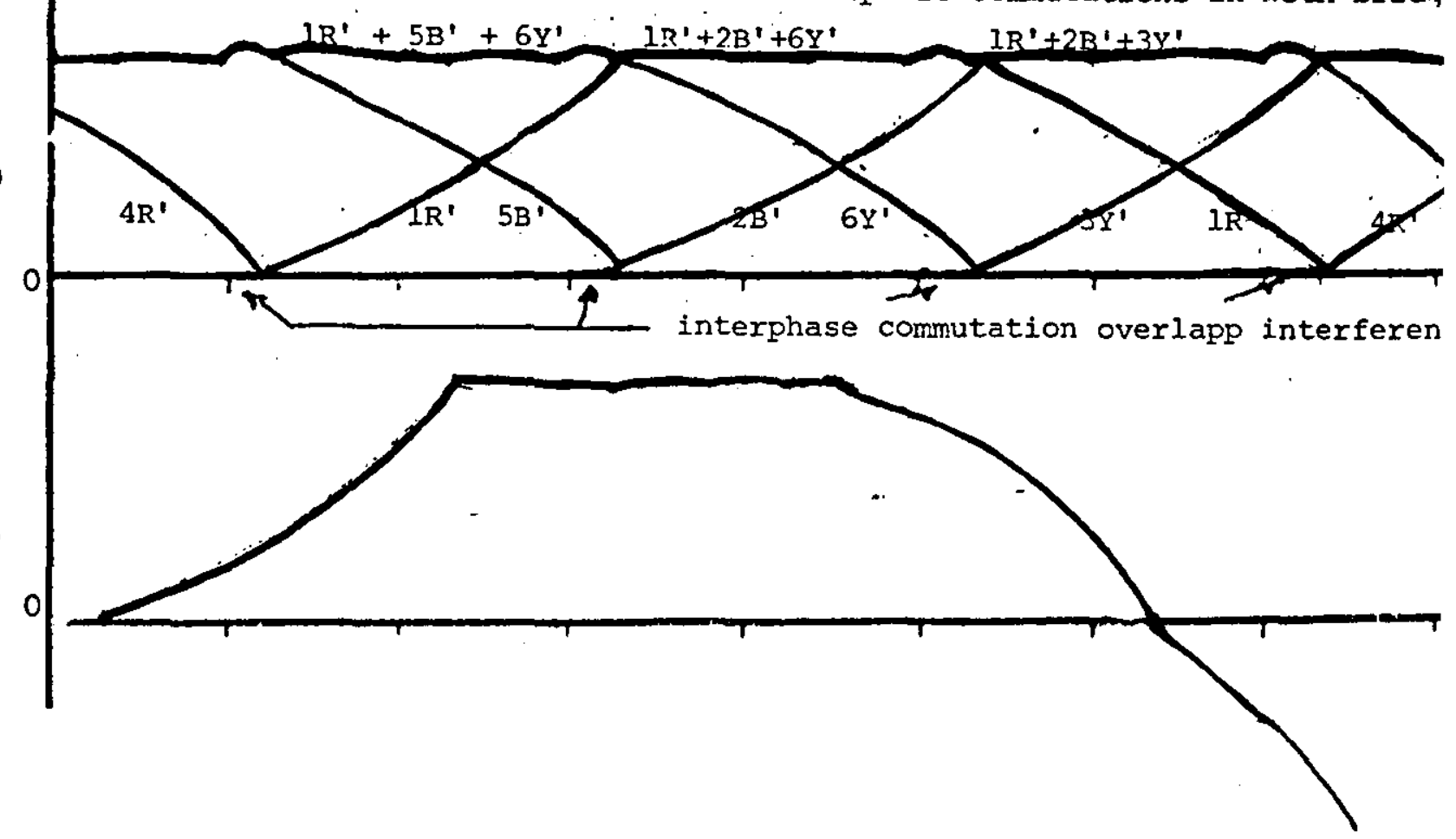

FIG. 2.13 Projected Mode 5 Waveforms

$(\lambda=$ angle of completion of coincidence of interphase commutations between the bridges always equal to $60^{\circ}$

Key: (a) dc output voltage

(d) bridge 2 current

(b) load current

(e) bridge 1 supply line

(c) bridae 1 current current I 
immediately after $6 \mathrm{Y}$ current has ceased to flow. Each thyristor conducts for $180^{\circ}$.

Interphase commutation angles approaching $60^{\circ}$ only occur under abnormally high overload conditions which could not be met on the laboratory test equipment. Nor is Mode 5 usual in industrial electrochemical practice, and so will not be considered further.

\subsection{Summary of the Operating Modes.}

The operating modes with interbridge and interphase commutations and their overlapping intervals are summarised in Table 2.1. 


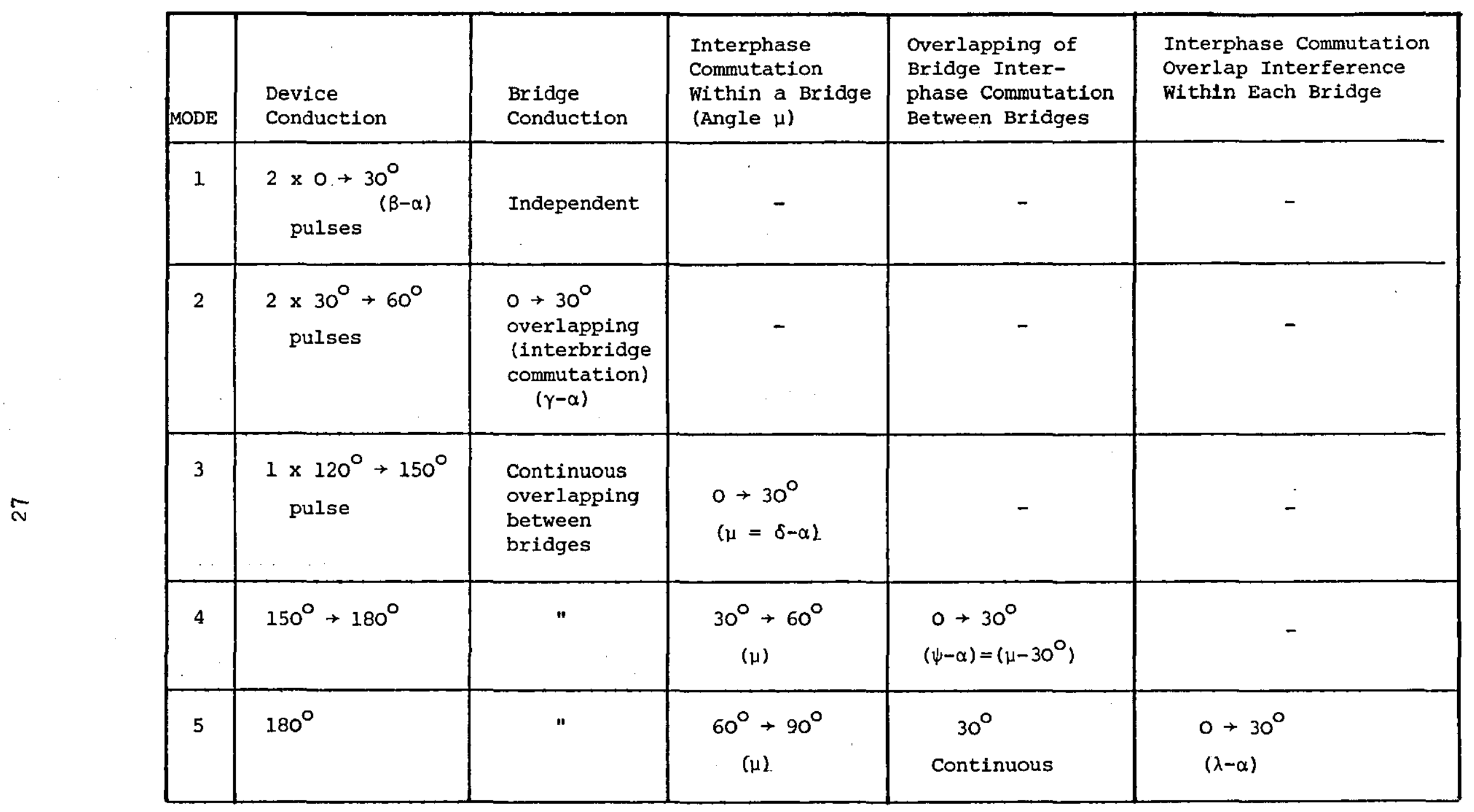

TABLE 2.1 SHOWING COMMUTATION ANGLES AND INTERACTION BETWEEN BRIDGES. 
CHAPTER 3

\subsection{Introduction}

The use of a digital computex to analyse rectifier circuits has resulted in various digital models of the circuit being presented. Both topological and tensor techniques are used by several authors. A typical one is known as the model-subroutine approach (ref: 7-11). This entails assigning a separate program subroutine to each of the sets of differential equations associated with the different circuit topologies (ref: 12). These solutions have, however, been confined to rectifier circuits where only a few sets of equations are involved. When many different permutations of differential equations are involved, the model-subroutine approach is cumbersome and wasteful of computer time. For example, for the 3-phase bridge, up to 25 sets of equations are required for a full analysis (ref: 7). Therefore a superior technique is required for which the work of Kron (ref: 15, 16) has proved to be useful. This employs tensor-analysis (ref: 12, 13), to define mesh currents as system state variables, and generates automatically the relevant differential equations as the device conduction patterns change. However, a variation of this approach has been developed by Kettleborough (ref; 14) that pertains to the handling of the change in device conduction patterns. This elegant technique will now be explained in relation to the 12-pulse parallel bridge system.

\section{3,2 Assumptions}

The assumptions made in the analysis are:

(i) Both the thyristor bridges are supplied by balanced, sinusoidal voltages with corresponding phase voltages, displaced by $30^{\circ}$. 
(ii) Each thyristor bridge has firing delay angle symetry.

(iii) Transformer impedances are perfectly balanced between phases and between the two secondary windings.

Finite values of load inductance, resistance, back e.m.f. and ac side resistance and inductance are allowed for, together with thyristor on-state impedance.

\subsection{Method}

The comprehensive technique described here involves tensor analysis, with the mesh currents defined as the system state variables. The connection tensors derlved from the transformer-thyristor circuits, are used directly to assemble the relevant mesh state variable equations, which are dependent on the thyristor conduction patterns.

The analysis involves an algorithm which,

(a). assembles automatically, for any thyristor conduction pattern, the closed path or mesh contour equations in state variable form (i.e. it sets up the mesh equations by transformation methods).

(bL solves the equations using a numerical integration method.

(cI. determines the individual branch currents and voltages from the mesh values.

Assembly of the mesh contour equations requires three reference frames:

(al the primitive or branch reference frame

(b) the intermediate reference frame

(c) the mesh reference frame

\subsection{The Transformer-Thyristor Unit}

Before describing the three reference frames it is necessary to derive the voltage equations for the transformer unit. The derivation 
of the complete equivalent representation of the transformer which includes the mutual inductances both between windings of the same phase and between phases has been presented in ref. 14. This develops the relationship between the inductances (self and mutual) with the physical quantities of the transformer, i.e. the inductance is expressed in terms of the number of winding turns and the reluctance of the core. This is not necessary here as the appropriate parameters for the transformer are obtained directly by measurement.

In general, the voltage equations derived for any windings of the 3-winding transformer can be written as follows, e.g. for winding 1 ,

$$
v_{1}=r_{1}{ }^{1}+L_{11} \frac{d i^{1}}{d t}+\sum_{n=2}^{9} L_{1 n} \frac{d i^{n}}{d t}
$$

where $\dddot{v}_{1}$ is the voltage across the terminals of the winding; $r_{1}$ is the winding resistance; $L_{11}$ is the self inductance of the winding and included in it is the leakage inductance $\ell_{11}$ and $I_{1 n}$ is the mutual inductance between windings. Corresponding equations may similarly be dexived for the other eight transformer windings.

As mentioned in the previous chapter, the unit used in the laboratory for the system comprises three separate single-phase 3-winding transformers. The arrangement is given in Fig. 3.1. The voltage equations for windings 1,4 and 7 as given generally by equation 3.1 , are:

$$
\begin{aligned}
& v_{1}=r_{1} i^{1}+L_{11} \frac{d i^{1}}{d t}+L_{14} \frac{d i^{4}}{d t}+L_{17} \frac{d i^{7}}{d t} \\
& v_{4}=r_{4} i^{4}+L_{44} \frac{d i^{4}}{d t}+L_{14} \frac{d i^{1}}{d t}+L_{47} \frac{d i^{7}}{d t} \\
& v_{7}=r_{7} i^{7}+L_{77} \frac{d i^{7}}{d t}+L_{17} \frac{d i^{1}}{d t}+L_{47} \frac{d i^{4}}{d t}
\end{aligned}
$$

The voltage equation for the other windings may be similarly represented. 


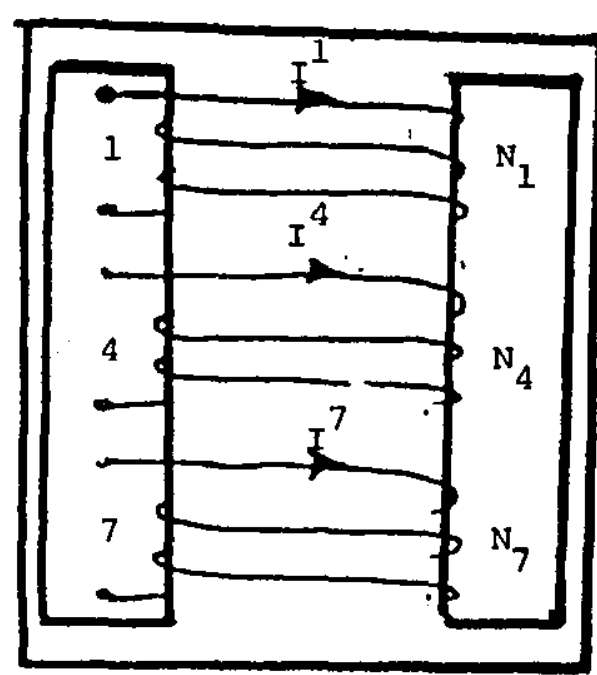

Phase A

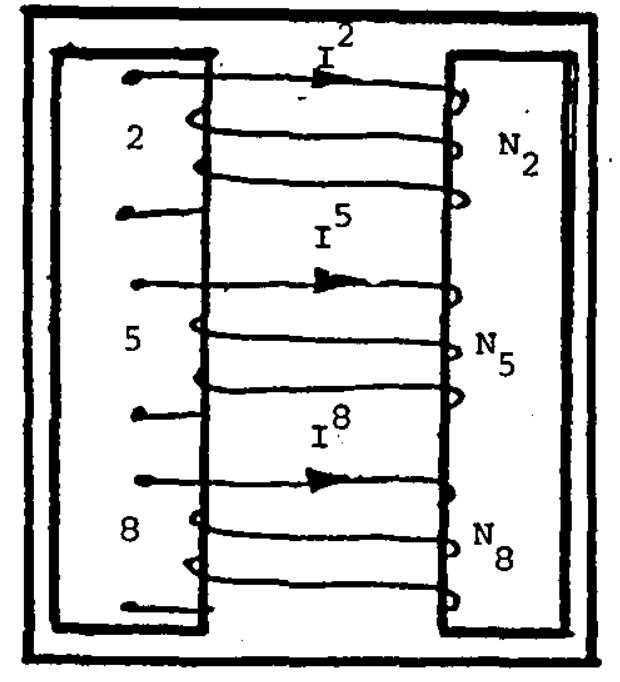

Phase B

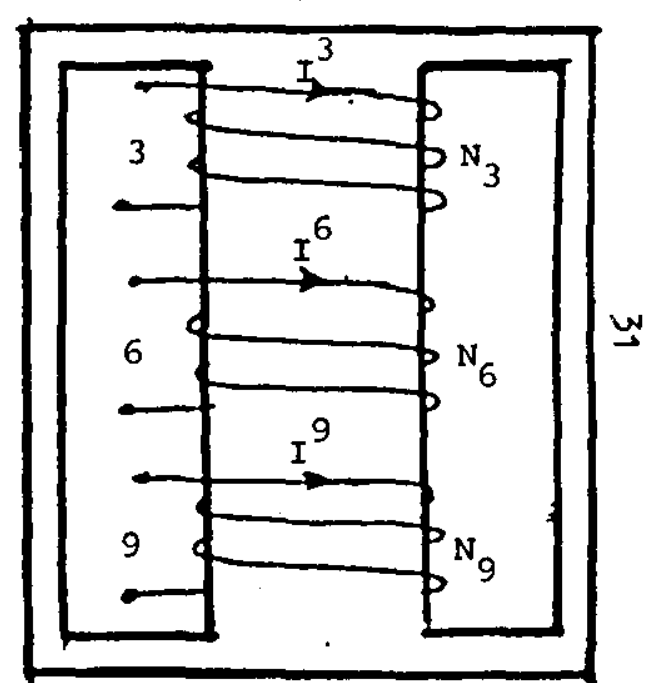

Phase C

FIG. 3.1 Schematic Dlagram of the Three winding Transformer 
From these equations, the mutual inductances between the phases are absent since separate single-phase transformers were used. However, these mutual inductances are included in the mathematical model for completeness, so that the technique is completely general. The measured values of the resistance and the self and mutual inductances of the three winding transformer are given in Chapter 6 .

In the analysis, the thyristors are assumed to have infinite impedance when non-conducting and a finite impedance with a threshold voltage when conducting. The latter is obtained from manufacturer's data.

\subsection{Modelling the System.}

\subsubsection{General.}

In this section, a complete mathematical model will be developed for the 12-pulse parallel bridge system using a tensor approach. The concept of tensor used in this modelling lies basically in the property of the tensor to relate its components in one reference frame to the components in another reference frame. Tensors are particularly useful when transforming from one reference frame to another because they represent relationships and characteristics which remain invariant in the transformation. (ref: 17). In particular, here, two significant tensors will be derived, one is fixed, $\mathrm{e}_{i}^{b}$, that is, there is no change in its components throughout the operation and the other is dynamic, $c .{ }_{m}^{i}$. The former relates the intermediate reference frame to the primitive reference frame. The latter, which is due to the application of Kron's approach, relates the mesh reference frame to 
the intermediate reference frame. The approach is known as Kron's "equations of constraint" (ref: 13, 15, 16) which provide a method that gives rise to the dynamic connecting tensors, $c_{m}^{i}$. This will be explained later.

The following subsections describe the three reference frames and the derivation of the two connecting tensors $c c_{i}^{b}$ and $c_{\cdot m}^{i}$. Happ's tensor notation is adopted (ref: 17), all impedances are operational and, unless otherwise stated, the equations are in matrix form. Also to note here that, although, in this work, the absence of the interbridge reactor is the main concern, it is included for completeness, so that the model can be used universally.

\subsubsection{The Primitive Reference Frame}

From the point of view of tensor analysis, any system to be analysed is first visualised in terms of a physically existing live network which consists of a series of branches such as those in Fig. 3.2 to Fig.3.5. This type of network is called a primitive network (ref: 15, 16), since it represents the building blocks of a more complex interconnected network.

The primitive network will serve as a "reference network". It is a particularly convenient reference network, since it is a conceptually easy network to visualise and to formulate, and its equations in terms of the $\mathrm{Z}$ or the $\mathrm{Y}$ matrix are generally known; furthermore the variables in the primitive network are usually of particular interest, since they constitute the solution that is generally desired (ref: 17).

The primitive reference frame for the parallel bridge rectifier system is given in Fig. 3.6. But before deriving its primitive 

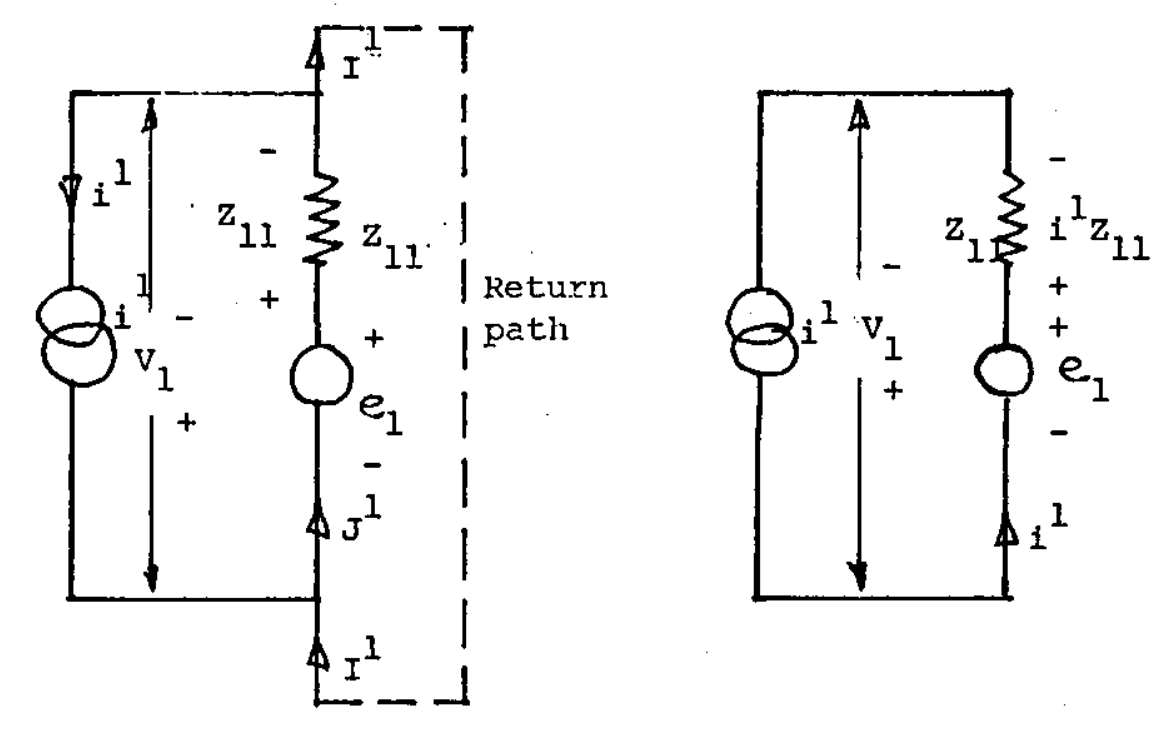

FIG. 3.2
FIG. 3.3

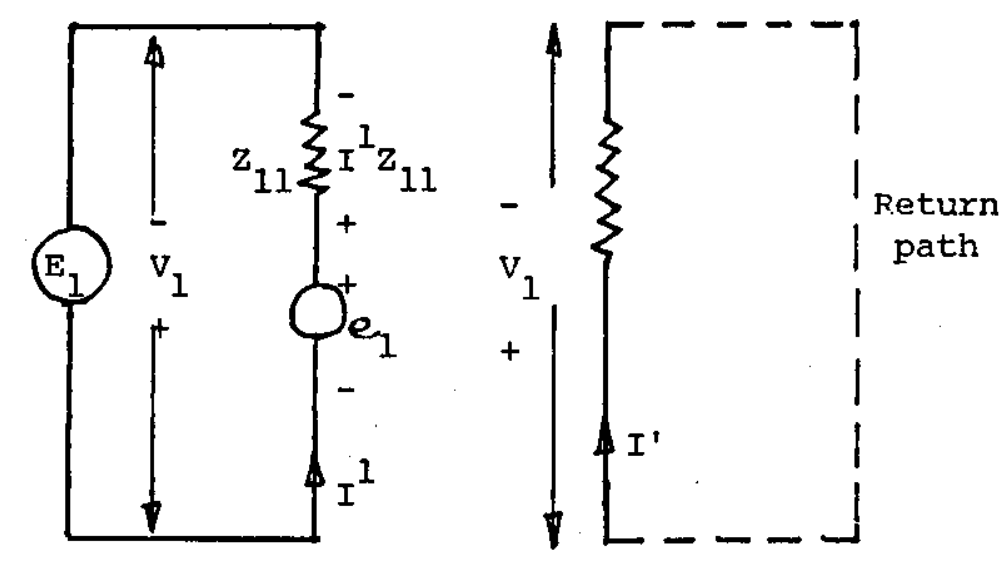

$\underset{\perp}{W}$

A Single Branch of the Primitive Network

FIG. 3.4

FIG. 3.5 
matrix equation, it is useful to get an insight into the generalised single branch network as depicted in fig. 3.2. As introduced by Happ, this network is expressed by the following equations,

$$
v_{1}+e_{1}=z_{11}\left(I^{1}+i^{1}\right)
$$

and

$$
J^{1}=I^{1}+i^{1}
$$

or more generally,

$$
v_{b}+e_{b}=z_{b b}\left(I^{b}+i^{b}\right)
$$

and

$$
J^{b}=I^{b}+i^{b}
$$

If the circuit of Fig. 3.2 is excited by a current generator $1^{1}$ connected across its extremities, $J^{1}$ will be entirely composed of $1^{1}$ as illustrated in Fig. 3.3. If a voltage source is connected across the extremities of the branch and no current sources are present, $1^{1}$ will be zero and $\mathrm{J}^{1}$ will be entirely composed of $\mathrm{I}^{1}$ as illustrated in Fig. 3.4. Similar reasoning may be imposed on the branch network of Fig. 3.2 with the situation where there is no current source or voltage source connected across its extremities and the active voltage source $v_{1}$ is absent. Fig. 3.5 illustrates this situation. Thus, the generalised equation representing this circuit is,

$$
v_{b}=z_{b b} I^{b}
$$

With this insight the primitive reference frame voltage equation can be derived and it is based on the primitive networks of Fig. 3.6. On the transformer side, the equation is given by;

$$
\mathrm{E}_{\mathrm{b}}=\mathrm{z}_{\mathrm{bb}} \mathrm{I}^{\mathrm{b}}
$$

for the primary winding, where each branch is connected at its extremities by the supply line voltage, $E_{b}$. For the secondaries, the tertiary and 


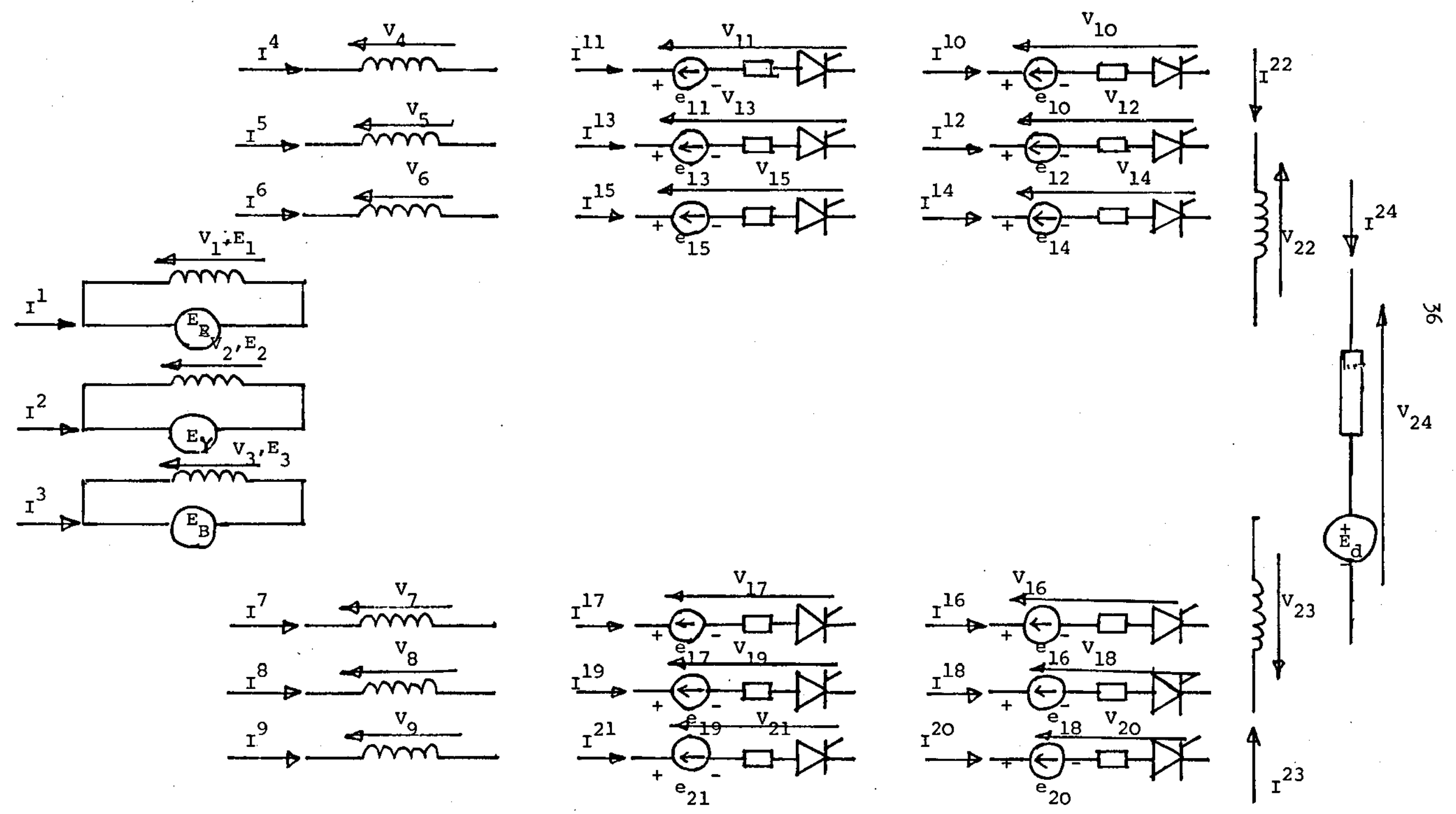

FIG. 3.6 The Primitive Reference Frame 
the star windings, the equation representing the networks is as given by eqn. 3.9 .

The primitive network of the thyristor circuit when the devices are conducting indicates a threshold voltage, say, $e_{11}$ in branch 11 which is analogous to the active voltage, $e_{1}$ of Fig. 3.2, but in the opposite sense. Thus the equation for branch 11 is given by,

$$
v_{11}-e_{11}=z_{1111} I^{11}
$$

or more generally,

$$
v_{b}-e_{b}=z_{b b} I^{b}
$$

Also, for the interbridge reactor primitive network, the generalised equation is that given by eqn. 3.9 , and for the load with the presence of the back e.m.f. $E_{d}$,

$$
\mathrm{v}_{\mathrm{b}}-\mathrm{E}_{\mathrm{a}}=\mathrm{z}_{\mathrm{bb}} \mathrm{I}^{\mathrm{b}}
$$

The matrix elements constituting equations 3.9 to 3.13 are given in Fig. 3.7a, b, and c. As can be seen, the main diagonal terms are resistances and operational inductances between the windings of each phase. Formulation of the impedance matrix is simple, as the transformer, thyristors, interbridge reactor and load are all represented by separate sub-matrices symmetrical about the main diagonal. This enables detailed information on the parameters of the constituent parts of the system to be obtained separately.

\subsubsection{The Intermediate Reference Frame}

Fig. 3.9 shows the complete winding connections of the transformer, and the permanent connections between thyristors and the connections between the interphase reactor and the load. Applying Kirchhof's current law to the nodes the corresponding currents are related as shown by the figure. For example, consider the node connecting 


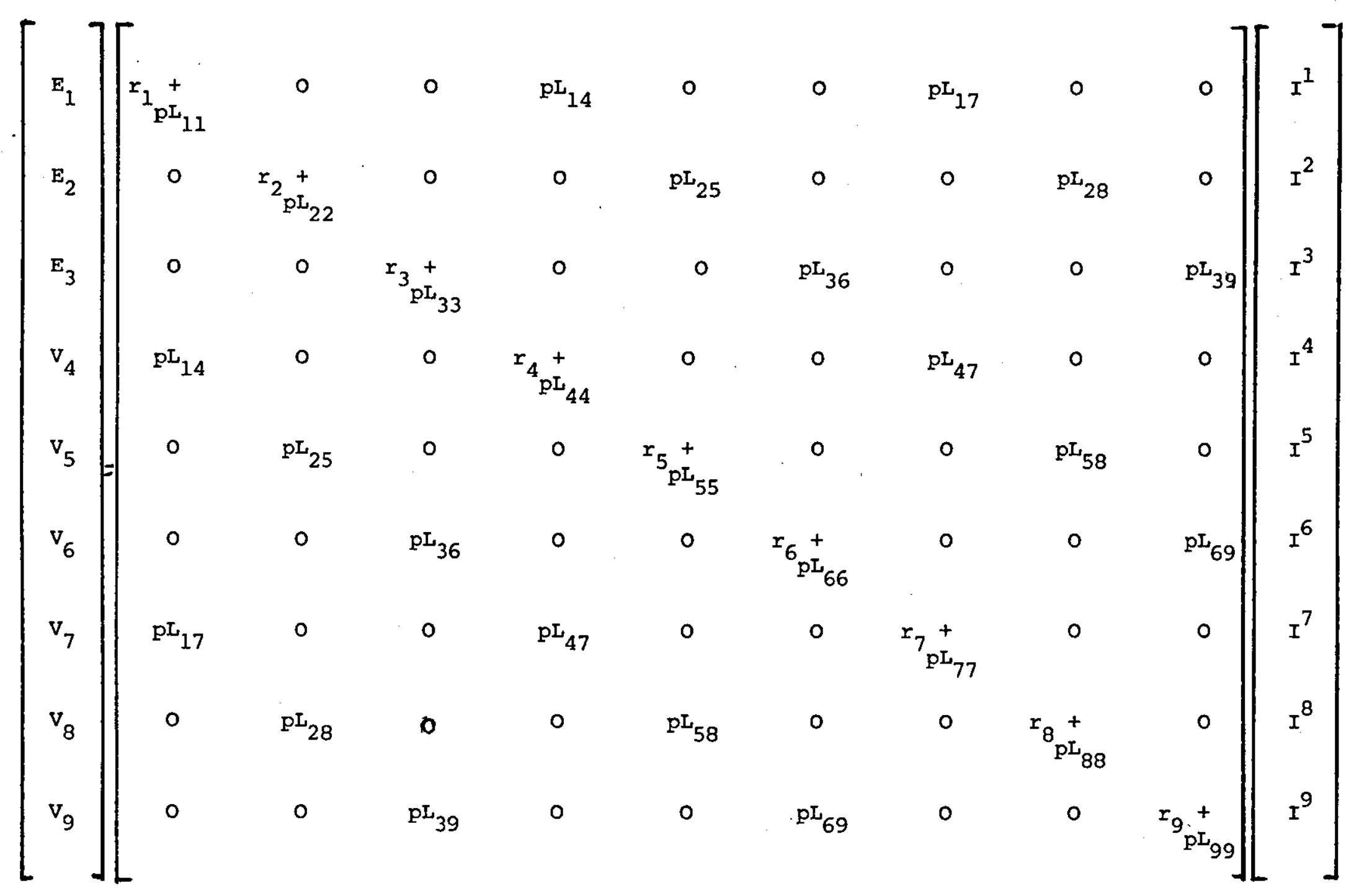

FIG. 3.7a PRIMITIVE REFERENCE FRAME TRANSFORMER VOLTAGE EQUATION 


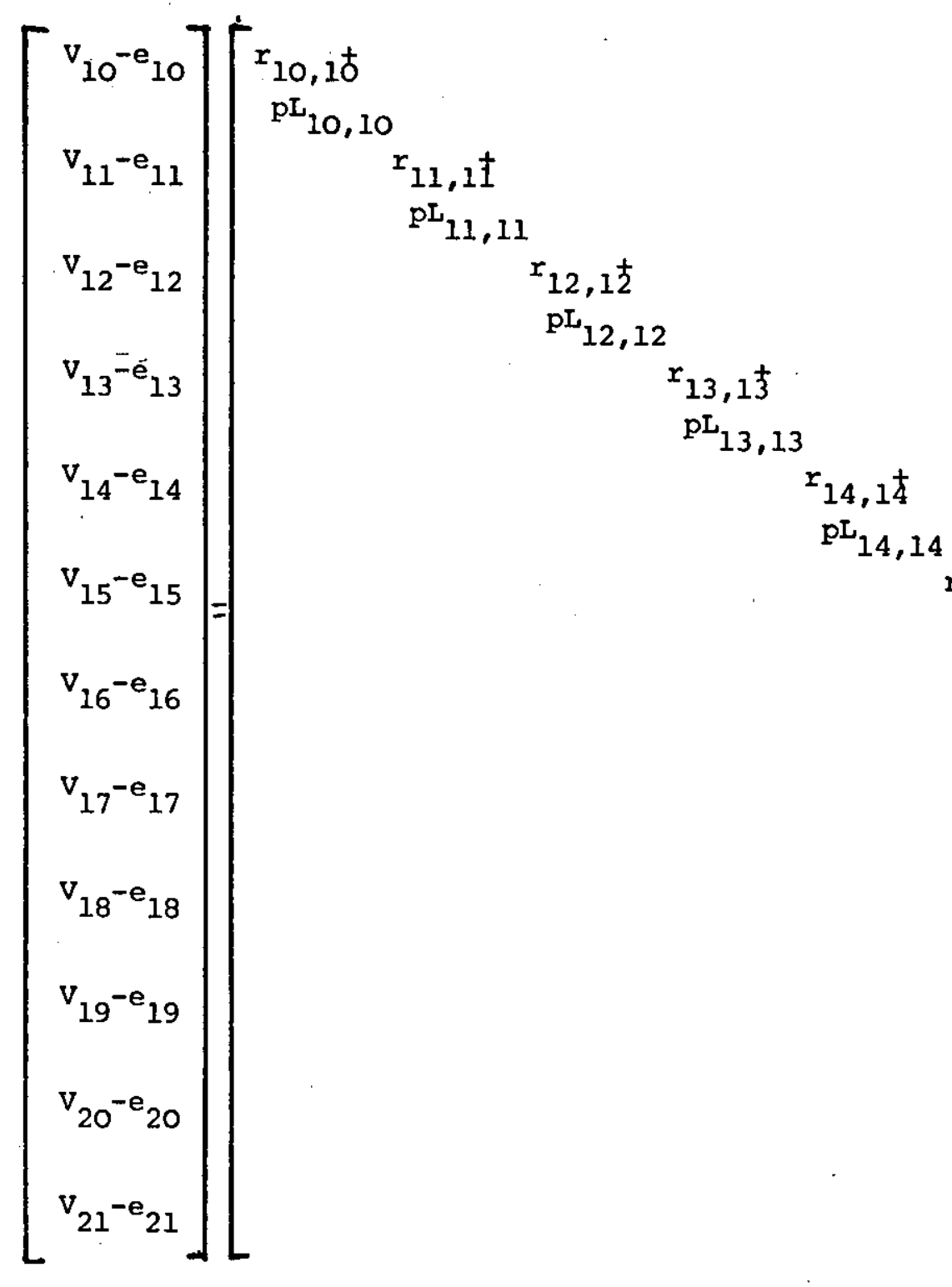

$r_{15,15}$
$\mathrm{pL}_{15,15}$

$r_{16,16}$

$\mathrm{pL}_{16,16}$

$r_{17,17}$

${ }^{\mathrm{pL}} 17,17$

$x_{18,18}$

$\mathrm{pL}_{18,18}$

$r_{19,19}$

$\mathrm{pL}_{19,19}$

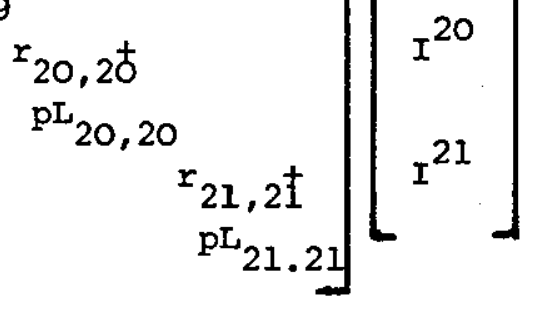

FIG. $3.7 \mathrm{~b}$ PRIMITIVE REFERENCE FRAME THYRISTORS VOLTAGE EQUATION 


$$
\left[\begin{array}{l}
\mathrm{v}_{22} \\
\mathrm{v}_{23} \\
\mathrm{v}_{24}-\mathrm{E}_{\mathrm{d}}
\end{array}\right]=\left[\begin{array}{ccc}
\mathrm{r}_{22,2 \frac{1}{2}} & \mathrm{pL}_{22,23} & 0 \\
\mathrm{pL}_{22,22} & & \\
& & \\
\mathrm{pL}_{22,23} & \begin{array}{c}
\mathrm{r}_{23,2 \frac{1}{3}} \\
\mathrm{pI}_{23,23}
\end{array} & 0 \\
0 & 0 & \mathrm{r}_{24,2 \frac{14}{2}} \\
& & \mathrm{pL}_{24.24}
\end{array}\right]\left[\begin{array}{l}
\mathrm{I}^{22} \\
\mathrm{I}^{23} \\
\mathrm{I}^{24}
\end{array}\right]
$$

FIG. 3.7c PRIMITIVE REFERENCE FRAME IPT AND LOAD VOLTAGE EQUATION 


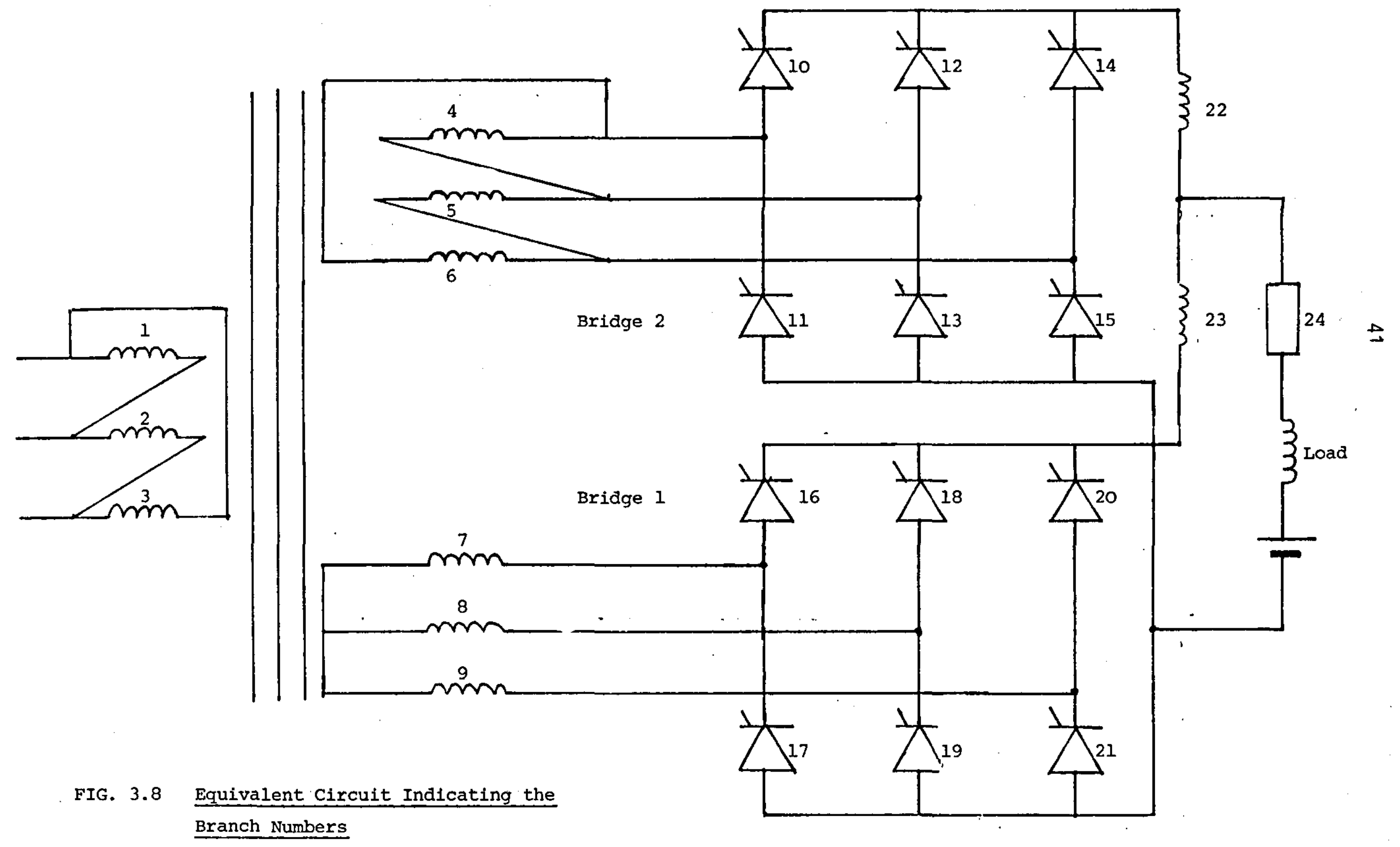




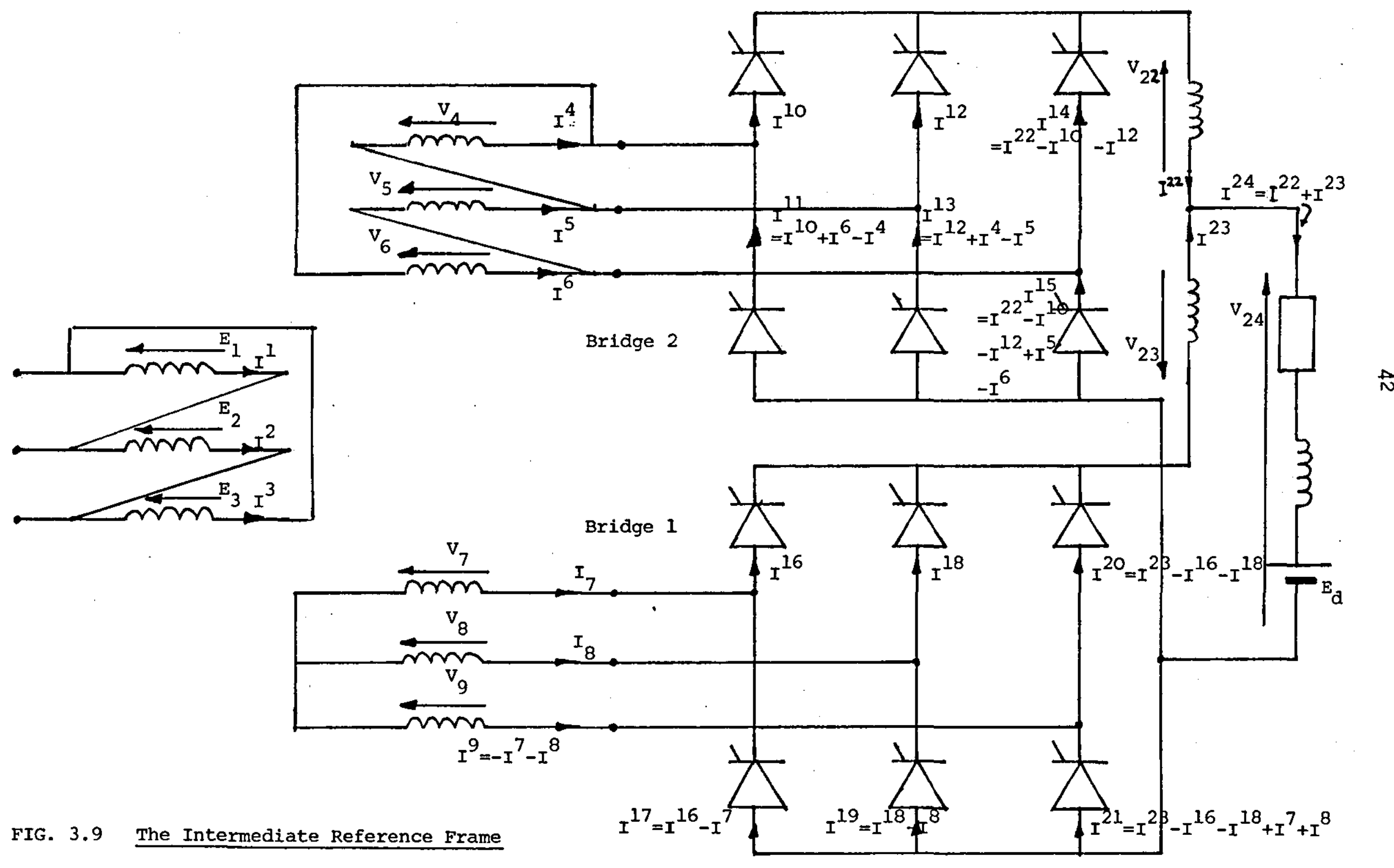


branches 4, 6, 10 and 11 (see Fig.3.8). Branch 11 has its current expressed as the other three, i.e. $I^{11}=I^{10}+I^{6}-I^{4}$. With the relationship of the currents shown in Fig. 3.9, a connection transformation matrix, $\mathrm{C}_{\mathrm{s}}^{\mathrm{b}}$ is established. It relates the primitive reference frame with the connected network as,

$$
I^{b}=c \cdot I_{s}^{b}
$$

This connecting tensor, $\mathrm{C}_{\mathrm{s}}^{\mathrm{b}}$ is shown in Fig. 3.10 .

Further simplification can be made with the connected network of Fig. 3.9 whereby the primary impedances are reflected to the two secondaries. In this situation, $I^{S}$ has to be rearranged such that a referred current transformation matrix, $c_{i}^{S}$ is obtained, where,

$$
I^{s}=C_{i}^{s} I^{i}
$$

Now, $I^{S}$ is derived in terms of $I^{i}$ by establishing the loop mmf equations for each phase, relating to the magnetic circuit of Fig. 3.1 . For this transformer type the coupling between windings of different phases is absent; as such the mmf terms due to the other two phases will not appear. in the equations below. However, it will appear in eqn. 3.15 as zero element in the matrix,.$_{i}^{s}$ (see Fig. 3.11). Thus the mmf equations in phase $A$,

$$
I^{l} N_{1}+I^{4} N_{4}+I^{7} N_{7}=0
$$

in phase $B$,

$$
I^{2} \mathrm{~N}_{2}+\mathrm{I}^{5} \mathrm{~N}_{5}+\mathrm{I}^{8} \mathrm{~N}_{8}=0
$$

and in phase $C$,

$$
I^{3} \mathrm{~N}_{3}+I^{6} \mathrm{~N}_{6}+I^{9} \mathrm{~N}_{9}=0
$$

As the windings in each phase are identical,

$$
\begin{aligned}
N_{1} & =N_{2}=N_{3} \\
N_{4} & =N_{5}=N_{6} \\
N_{7} & =N_{8}=N_{9} \\
\text { also } 1^{9} & =-I^{7}-I^{8}
\end{aligned}
$$




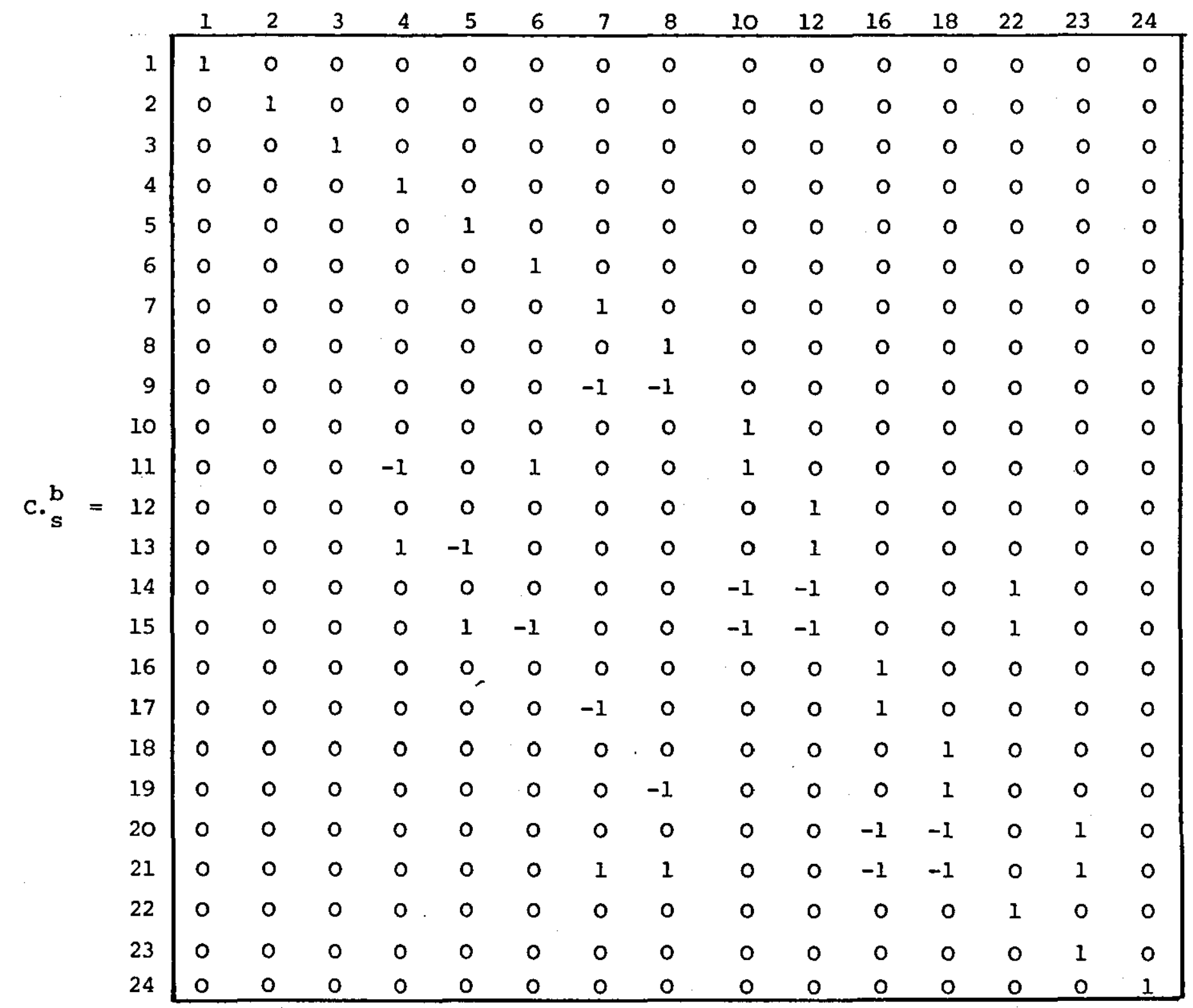




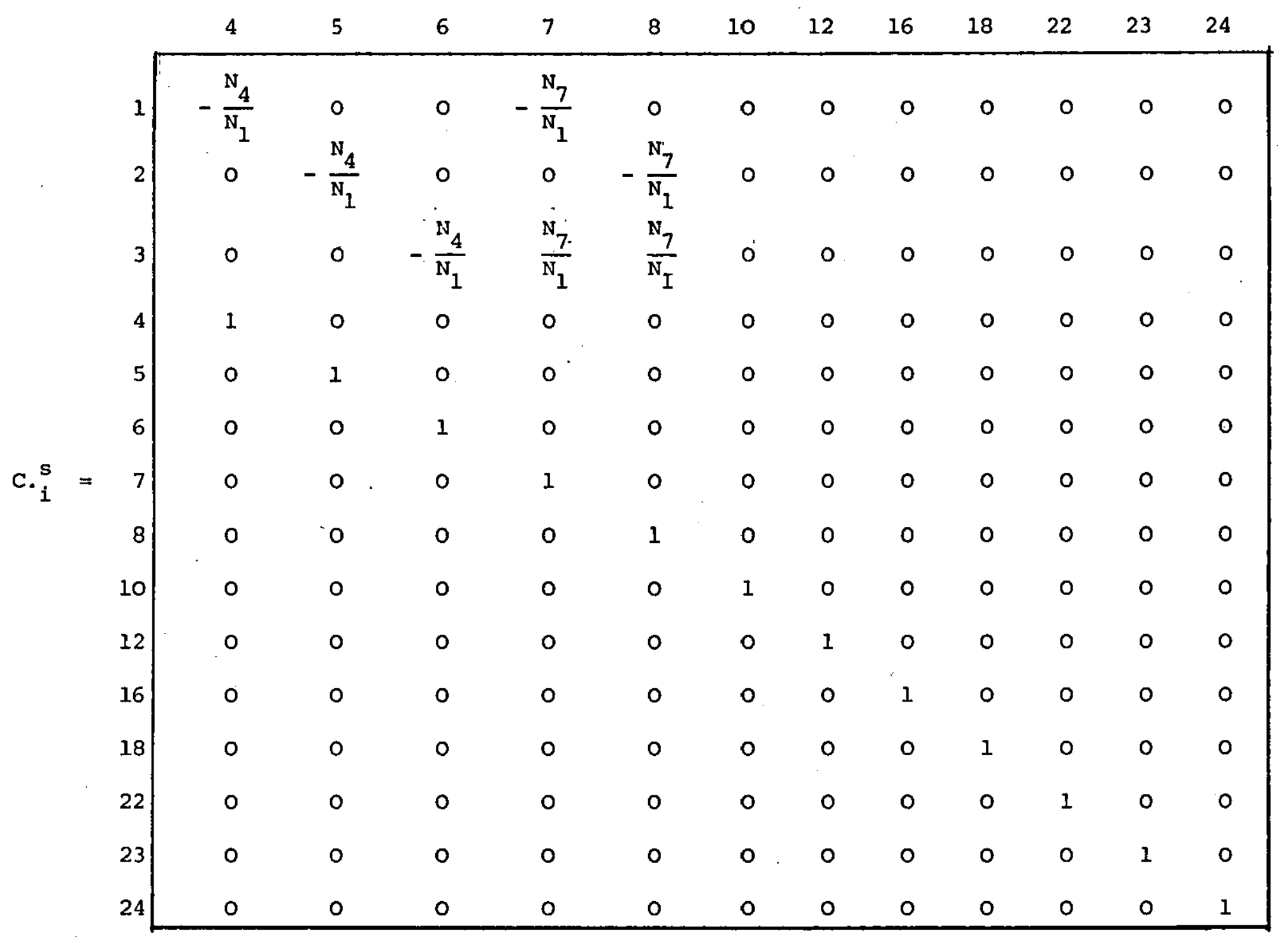

FIG. 3.11 REFERRED CURRENT TRANSFOFMATION MATRIX 
Rearranging and solving for $I^{1}, I^{2}$, and $I^{3}$,

$$
\begin{aligned}
& I^{2}=-I^{4} \frac{N_{4}}{N_{1}}-I^{7} \frac{N_{7}}{N_{1}} \\
& I^{2}=-I^{5} \frac{N_{4}}{N_{1}}-I^{8} \frac{N_{7}}{N_{1}} \\
& I^{3}=-I^{6} \frac{N_{4}}{N_{1}}+I^{7} \frac{N_{7}}{N_{1}}+I^{8} \frac{N_{7}}{N_{1}}
\end{aligned}
$$

This is true for a delta connected primary. As for the star winding, see Ref. 1.4. For the rest of $I^{\mathrm{S}}$, it is simply equal to $I^{1}$. Thus, the referred current transformation matrix is assembled as shown in Fig. 3.11 .

If equation 3.15 is premultiplied by $c . \frac{b}{s}$ then, and from eqn. 3.14 ,

$$
I^{b}=c \cdot{ }_{s}^{b} c \cdot{ }_{i}^{s} I^{i}
$$

From equation 3.23, the multiplication of $\mathrm{c}_{\mathrm{s}}^{\mathrm{b}}$ and $\mathrm{c}_{i}{ }_{i}^{\mathrm{s}}$ gives a new matrix that relates the primitive reference frame to the referred interconnected reference frame. This matrix is known as $c_{.}^{b}$ and is shown in Fig. 3.12. i.e.

$$
c \cdot{ }_{1}^{b}=c \cdot b \cdot s \cdot s
$$

The equation relating the referred interconnected branch voltages and currents are:

$$
E_{i}+v_{i}=z_{i i} I^{i}
$$

Assuming power invariance between the two reference frames, the following relationships apply:

$$
\begin{aligned}
& I^{b}=c \cdot \cdot_{i}^{b} I^{i} \\
& v_{i}=c_{i}^{\cdot b} v_{b} \\
& E_{i}=c_{i}^{\cdot b} E_{b} \\
& z_{i i}=c_{i}^{\cdot b} z_{b b} c \cdot{ }_{i}^{b}
\end{aligned}
$$




\begin{tabular}{|c|c|c|c|c|c|c|c|c|c|c|c|}
\hline 4 & 5 & 6 & 7 & 8 & 10 & 12 & 16 & 18 & 22 & 23 & 24 \\
\hline$-\frac{\mathrm{N}_{4}}{\mathrm{~N}_{1}}$ & 0 & 0 & $-\frac{N_{7}}{N_{1}}$ & 0 & 0 & 0 & 0 & 0 & 0 & 0 & 0 \\
\hline 0 & $-\frac{N_{4}}{N_{1}}$ & 0 & 0 & $-\frac{N_{7}}{N_{1}}$ & 0 & 0 & 0 & 0 & 0 & 0 & 0 \\
\hline 0 & 0 & $-\frac{\mathrm{N}_{4}}{\mathrm{~N}_{1}}$ & $\frac{\mathrm{N}_{7}}{\mathrm{~N}_{1}}$ & $\frac{\mathrm{N}_{7}}{\mathrm{~N}_{1}}$ & 0 & 0 & 0 & 0 & 0 & 0 & 0 \\
\hline 1 & 0 & 0 & 0 & 0 & 0 & 0 & 0 & 0 & 0 & 0 & 0 \\
\hline 0 & 1 & 0 & 0 & 0 & 0 & 0 & 0 & 0 & 0 & 0 & 0 \\
\hline 0 & 0 & 1 & 0 & 0 & 0 & 0 & 0 & 0 & 0 & 0 & 0 \\
\hline 0 & 0 & 0 & 1 & 0 & 0 & 0 & 0 & 0 & 0 & 0 & 0 \\
\hline 0 & 0 & 0 & 0 & 1 & 0 & 0 & 0 & 0 & 0 & 0 & 0 \\
\hline 0 & 0 & 0 & -1 & -1 & 0 & 0 & 0 & 0 & 0 & 0 & 0 \\
\hline 10 & 0 & 0 & 0 & 0 & 1 & 0 & 0 & 0 & 0 & 0 & 0 \\
\hline-1 & 0 & 1 & 0 & 0 & 1 & 0 & 0 & 0 & 0 & 0 & 0 \\
\hline 12 & 0 & 0 & 0 & 0 & 0 & 1 & 0 & 0 & 0 & 0 & 0 \\
\hline 13 & -1 & 0 & 0 & 0 & 0 & 1 & 0 & 0 & 0 & 0 & 0 \\
\hline 14 & 0 & 0 & 0 & 0 & -1 & -1 & 0 & 0 & 1 & 0 & 0 \\
\hline 15 & 1 & -1 & 0 & 0 & -1 & -1 & 0 & 0 & 1 & 0 & 0 \\
\hline 16 & 0 & 0 & 0 & 0 & 0 & 0 & 1 & 0 & 0 & 0 & 0 \\
\hline 17 & 0 & 0 & -1 & 0 & 0 & 0 & 1 & 0 & 0 & 0 & 0 \\
\hline 18 & 0 & 0 & 0 & 0 & 0 & 0 & 0 & 1 & 0 & 0 & 0 \\
\hline 19 & 0 & 0 & 0 & -1 & 0 & 0 & 0 & 1 & 0 & 0 & $\therefore$ \\
\hline 20 & 0 & 0 & 0 & 0 & 0 & 0 & -1 & -1 & 0 & 1 & 0 \\
\hline 21 & 0 & 0 & 1 & 1 & 0 & 0 & -1 & -1 & 0 & 1 & 0 \\
\hline 22 & 0 & 0 & 0 & 0 & 0 & 0 & 0 & 0 & 1 & 0 & 0 \\
\hline 23 & 0 & 0 & 0 & 0 & 0 & 0 & 0 & 0 & 0 & 1 & 0 \\
\hline 24 & 0 & 0 & 0 & 0 & 0 & 0 & 0 & 0 & 0 & 0 & 1 \\
\hline
\end{tabular}

FIG. 3.12 TRANSFORMATION MATRIX $c_{i}^{b}$ 


\subsubsection{The Mesh Reference Frame}

In this final stage of the mathematical modelling, the change in state of any of the thyristors is catered for. It was mentioned previously that Kron's "equation of constraint" technique provides the method for this, which is valid after the topology change. This approach simply imposes certain constraints on the device current, i.e. the currents are set to zero. It follows from this that a new connecting matrix is then developed which relates the intermediate reference frame to the new mesh reference frame due to the topology change. This new connecting matrix is in fact a reduction of the matrix, $c_{i}^{b}$. This tensor or matrix reduction method can be applied systematically to produce the new state equations following any convertor topology change, (ref. 13).

A slight variation of the approach mentioned above, however, has been adopted here. This takes into consideration the mesh currents of the circuit that result from the topology change. As such the currents of the non-conducting devices are neglected altogether, i.e. without putting them to zero which eases computer implementation. The number of mesh currents needed for a particular topology change is simply obtained from the following topological relationship, (ref.17),

$$
M=B-N+S
$$

where $\mathrm{M}$ is the minimum number of independent meshes, or closed paths; $B$ is the number of branches; $N$ is the number of nodes and $S$ is the number of electrically isolated subnetworks and in this case $S=1$. A particular topological change of the parallel bridge will be cited to explain this situation.

If, in the circuit of Fig. 3.8, only devices 10, 13, 16 and 19 are conducting, according to equation 3.30, three independent mesh 
currents are needed which is illustrated by Fig. 2.6. Taking the current orientation shown by this figure, the current of the intermediate reference frame $I^{i}$ is related to the three loop currents $i_{,} i_{a}$ and $i_{b}$ by the matrix, c. ${ }_{m}^{i}$ as follows,

$$
\left[\begin{array}{l}
I^{4} \\
I^{5} \\
I^{6} \\
I^{7} \\
I^{8} \\
I^{10} \\
I^{12} \\
I^{16} \\
I^{18} \\
I^{22} \\
I^{23} \\
I^{24}
\end{array}\right]=\left[\begin{array}{lll}
I & 1 & 0 \\
1 & 0 & 0 \\
1 & 0 & 0 \\
0 & 0 & 1 \\
0 & 0 & -1 \\
0 & 1 & 0 \\
0 & 0 & 0 \\
0 & 0 & 1 \\
0 & 0 & 0 \\
0 & 1 & 0 \\
0 & 0 & 1 \\
i^{b}
\end{array}\right]
$$

The connecting matrix, $\mathrm{c}_{\mathrm{m}}^{1}$ is part of the master thyristor conduction matrix which will be derived in the following section. In general, the matrix equation relating the mesh currents and voltages for these loops and the permanent loop of the transformer tertiary winding is,

$$
\begin{aligned}
E_{m}+V_{m} & =z_{m m} I^{m} \\
& =\left(R_{m m}+p L_{m m}\right) I^{m}
\end{aligned}
$$

As shown by Kirchoff's voltage law, the vector, $v_{m}$ is zero.

In a manner similar to that of the previous section, the following relationships apply between the mesh and the intermediate reference frames: 


$$
\begin{aligned}
I^{1} & =c \cdot \cdot_{m}^{i} I^{m} \\
v_{m} & =c_{m}^{\cdot i} v_{1} \\
E_{m} & =c_{m}^{\cdot i} E_{1} \\
z_{m m} & =c_{m}^{\cdot i} z_{i 1} c \cdot_{m}^{i}
\end{aligned}
$$

\subsubsection{The Master Thyristor Conduction Matrix}

The master thyristor conduction matrix is defined in Fig. 3.13. It consists of twelve loops to cater for all practical thyristor conduction patterns. Each loop contains one thyristor in the top and one in the bottom of a bridge, and is orientated in the direction of positive thyristor current to enable thyristor current extinction to be determined by monitoring the reversal of loop currents. The orientations of the various branches in the branch/mesh transformation matrix are obtained from the linear orientated graph of the system shown in Fig. 3.14. The following orientation of the graph branches is observed:

(a) from 'start' to 'finish' in transformer winding branches,

(b) In the direction of positive thyristor currents in the thyristor branches,

(c) in the direction of positive direct current on the dc sides of the two bridges.

\subsubsection{Derivation of the Transformation Matrix C. ${ }_{-m}^{i}$}

The transformation matrix, $c_{m}^{i}$ is dynamic, since its elements vary according to the various meshes generated by the different conducting thyristors. For example, consider Mode 4 operation, where conducting thyristors are shown in Fig. 2.10. The transformation matrix $\mathrm{c}_{\mathrm{m}}^{i}$ for these mesh current loops are assembled automatically by the computer program from the master transformation matrix of Fig. 3.13. The manner that this is done will be dealt with in Chapter 4. 


\begin{tabular}{|c|rrrrrrrrrrrrr|}
\hline $\begin{array}{l}\text { loop } \rightarrow \\
\text { Branch } \\
\text { No. }\end{array}$ & a & b & $c$ & $d$ & e & f & $g$ & $h$ & $i$ & $j$ & $k$ & 1 \\
\hline 4 & 1 & 0 & 0 & -1 & 0 & 0 & 0 & 0 & 0 & 0 & 0 & 0 \\
5 & 0 & 0 & 1 & 0 & 0 & -1 & 0 & 0 & 0 & 0 & 0 & 0 \\
6 & 0 & -1 & 0 & 0 & 1 & 0 & 0 & 0 & 0 & 0 & 0 & 0 \\
7 & 0 & 0 & 0 & 0 & 0 & 0 & 1 & 1 & 0 & -1 & -1 & 0 \\
8 & 0 & 0 & 0 & 0 & 0 & 0 & -1 & 0 & 1 & 1 & 0 & -1 \\
10 & 1 & 1 & 0 & 0 & 0 & 0 & 0 & 0 & 0 & 0 & 0 & 0 \\
12 & 0 & 0 & 1 & 1 & 0 & 0 & 0 & 0 & 0 & 0 & 0 & 0 \\
16 & 0 & 0 & 0 & 0 & 0 & 0 & 1 & 1 & 0 & 0 & 0 & 0 \\
18 & 0 & 0 & 0 & 0 & 0 & 0 & 0 & 0 & 1 & 1 & 0 & 0 \\
22 & 1 & 1 & 1 & 1 & 1 & 1 & 0 & 0 & 0 & 0 & 0 & 0 \\
23 & 0 & 0 & 0 & 0 & 0 & 0 & 1 & 1 & 1 & 1 & 1 & 1 \\
24 & 1 & 1 & 1 & 1 & 1 & 1 & 1 & 1 & 1 & 1 & 1 & 1 \\
\hline
\end{tabular}

Note: Other branches are omitted here as their effect is included in the electrical connections transformation matrix.

FIG. 3.13 BRANCH/MESH TRANSFORMATION MATRIX 
w

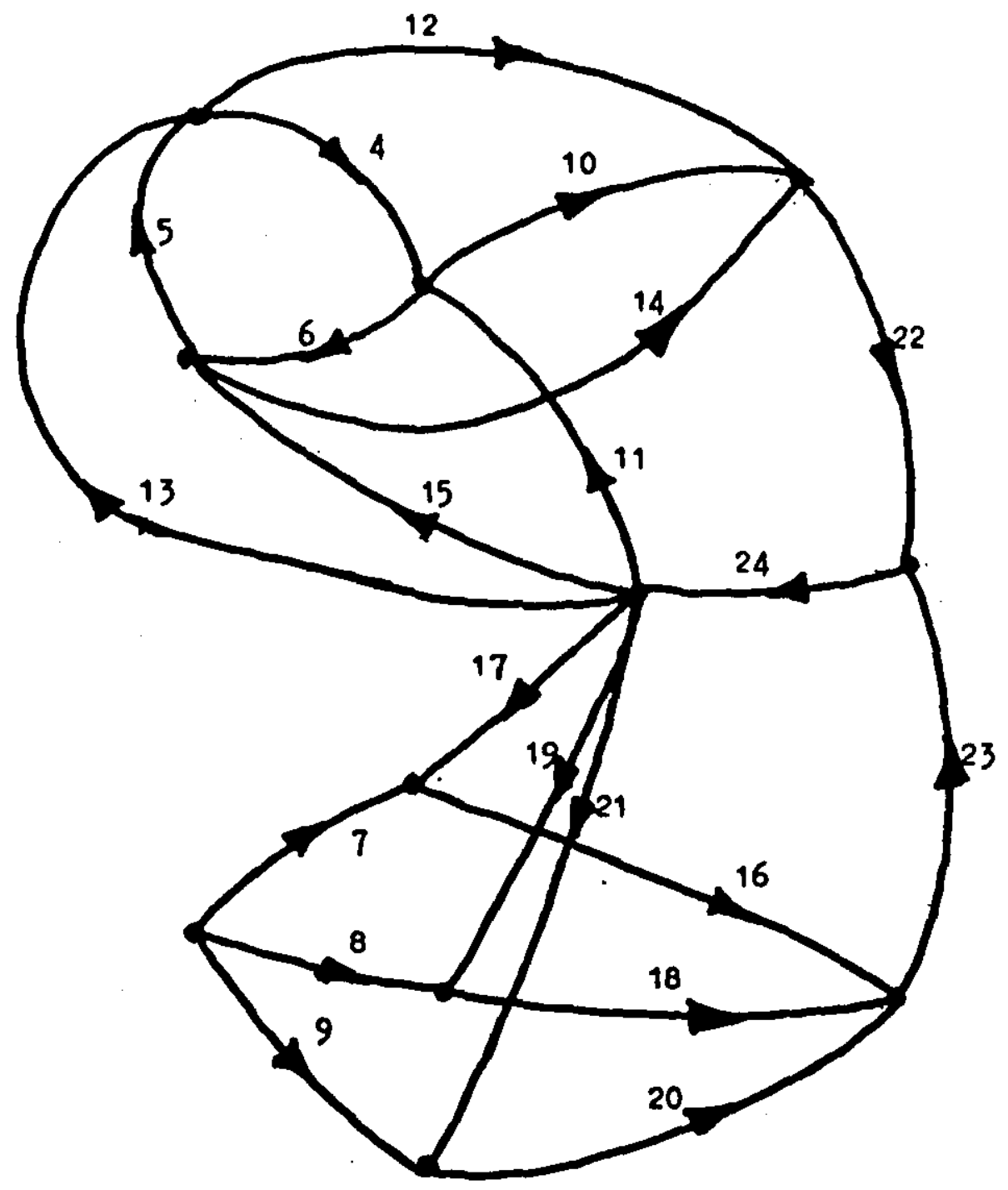

Note:

1. Branchen correspond to clrcult elements shown in $\mathrm{N1g} \cdot 3.8$

2. Primary branches are neglected since these elewents are referred into the econdary and tertlary windings of the transforwer.

F1G. 3.14 Linear orlentated Braph of the reduced system 


\subsection{Introduction}

An existing computer program developed originally for solving numerically the differential equations for a transformer-rectifiex unit (ref. 14) has been adapted and developed for this analysis. The unit considered in ref. 14 used diodes and an interbridge reactor. However, for the purpose of the present work, this computer program has incorporated the following additional features,

(a) a delta primary connection instead of star;

(b) formulation of triggering pulses for the thyristors and the introduction of firing delay to replace the diodes;

(c) the impedance of the interbridge reactor is set to zero;

(d) inclusion of the back emf in the load.circuit;

(e) inclusion of circuit elements to allow for thyristor voltage drop during conduction.

The integration step length has been chosen to give acceptable accuracy of results consistent with minimising computer run time. It has been established that the optimum step length for the RungeKutta integration procedure is equal to the shortest time constant of the system involved (ref.13). This will be dealt with further when discussing the integration technique used in the computer program. Fox an insight into the development of the computer program the sections below discuss the implementation of the mathematical model developed in Chapter 3. Firstly, the solution process is laid out Indicating the quantities needed to be calculated as well as the necessary procedures to be performed following any discontinuities or topological change. This is then followed by an illustration of 
a particular conducting situation of the thyristors as outlined in Chapter 2. Finally, the various computer subroutines and their specific functions are presented, and their uses related to the main program.

The computer program is written in FORTRAN. Lower case letters are used in the listings of Appendices III and IV rather than the normal upper case in accordance with the Honeywell MULTICS System. (ref.18) Subroutine names and variable arrays are shown in Capital letters in the following sections. The program provides the transformer winding, ac supply current and output voltage waveforms, both in numerical form and plotted, and their harmonic components.

\subsection{The Main Program}

The following series of operations describe the solution process of the computer program;

(a) Determine $z_{i i}$ (this matrix is constant, and has only to be assembled once at the beginning of a computer runl

$$
z_{i i}=c_{i}^{\cdot b} z_{b b} c \cdot \stackrel{b}{i}
$$

(b). Determine $z_{\mathrm{mm}}$ (this matrix is dynamic, and has to be reassembled when changes in network topology are caused by thyristor turn-on or turn-off).

$$
z_{m m}=C{ }_{m}^{\cdot i} z_{i i} \cdot \cdot_{m}^{i}
$$

(c) Determine $\mathrm{E}_{\mathrm{b}}$ and from it determine $\mathrm{E}_{\mathbf{i}}$

$$
E_{i}=c \cdot b E_{i}
$$

(d). Determine $\mathrm{E}_{\mathrm{m}}$

$$
E_{m}=c_{m}^{i} E_{i}
$$


(e) Equation 3.31 may be rearranged to give the state variable equation

$$
\mathrm{pI}^{\mathrm{m}}=\mathrm{L}_{\mathrm{mm}}^{-1}\left(\mathrm{E}_{\mathrm{m}}-\mathrm{R}_{\mathrm{mm}} \mathrm{I}^{\mathrm{m}}\right)
$$

Numerical integration yields the mesh current matrix $I^{m}$

(f) Determine $I^{i}$

$$
I^{i}=C \cdot \underbrace{i}_{m} I^{m}
$$

(g) Determine $I^{b}$ and $V_{b}$

$$
\begin{aligned}
& I^{b}=c \cdot \cdot_{i}^{b} I^{i} \\
& v_{b}=z_{b b} I^{b}-E_{b}
\end{aligned}
$$

The solution advances by one interval or step each time operations (c) to (g) are implemented. At the end of each step, the system is tested for any change in topology which may have occurred. If changes are detected, the solution proceeds as follows:

(1). the time between the start of the step and the first discontinuity (either a trigger pulse or loop current falling to zerol is determined; more than one discontinuity may occur within the step,

(ii) the state variable equation is integrated from the start of the step to the discontinuity,

(iii) the connection matrix, $\mathrm{c}_{\mathrm{m}}^{i}$ is re-assembled in accordance with the new system of meshes and its transpose $c_{\mathrm{m}}{ }_{\mathrm{m}}^{i}$ is formed,

(iv) the new mesh impedance matrix $z_{m m}$ is determined as outlined in operation (b) and the new state variable equation is formed,

(v) the new equation is integrated from the point of discontinuity to the end of the step, 
(vi) the system is tested for discontinuities occurring during the reduced step operation (v). If there is any change, operation (i) to (vi) are repeated. If no changes are found, the solution proceeds with operations $(c)$ to $(g)$ in the next step.

A simplified flow chart of the computer programs of Appendix III and IV are given respectively in Figs. $4.1 \mathrm{a}$ and $4.1 \mathrm{~b}$.

\subsection{Solution Process}

To illustrate the solution process, a typical device conduction pattern of the parallel convertor circuit is considered. Fig. 2.6 shows devices $I R$ and $6 Y$ conducting in bridge $I$ and devices $1 R^{\prime}$ and $6 Y^{\prime}$ conducting in bridge 2. The illustration here is made with reference to the computer program of Appendix IV.

Initially, the program determines the coefficients of the $\mathrm{z}_{\mathrm{bb}}$ matrix defined in Fig. 3.7a, $b$ and c. Subroutine CONST performs this function by determining the two 2-dimensional arrays $\mathrm{RB}$ and $\mathrm{XB}$, which are respectively the branch resistance and inductance matrices. Following this, and after obtaining the necessary input data, subroutine START determines the pair of thyristors that will conduct in each bridge. This is done by the systematic checking of all thyristors to find those which are most positively biased, and subroutine. CDUCTS provides the device forward conduction voltage drop. The elements of the connection matrix, $c_{i}^{b}$ of Fig. 3.12 and the master conduction matrix of Fig. 3.13 are located respectively in the 2-dimensional arrays CTRAN and CBRAN. Following the calling of subroutine LOAD, $c_{i}^{b}$ (CTRANT in the computer program) is assembled from CTRAN and $c ._{m}^{i}$ and $C_{m}^{. i}$, respectively $C B$ and $C B T$ in the program, are assembled from CBRAN. The latter transformations related, in this 


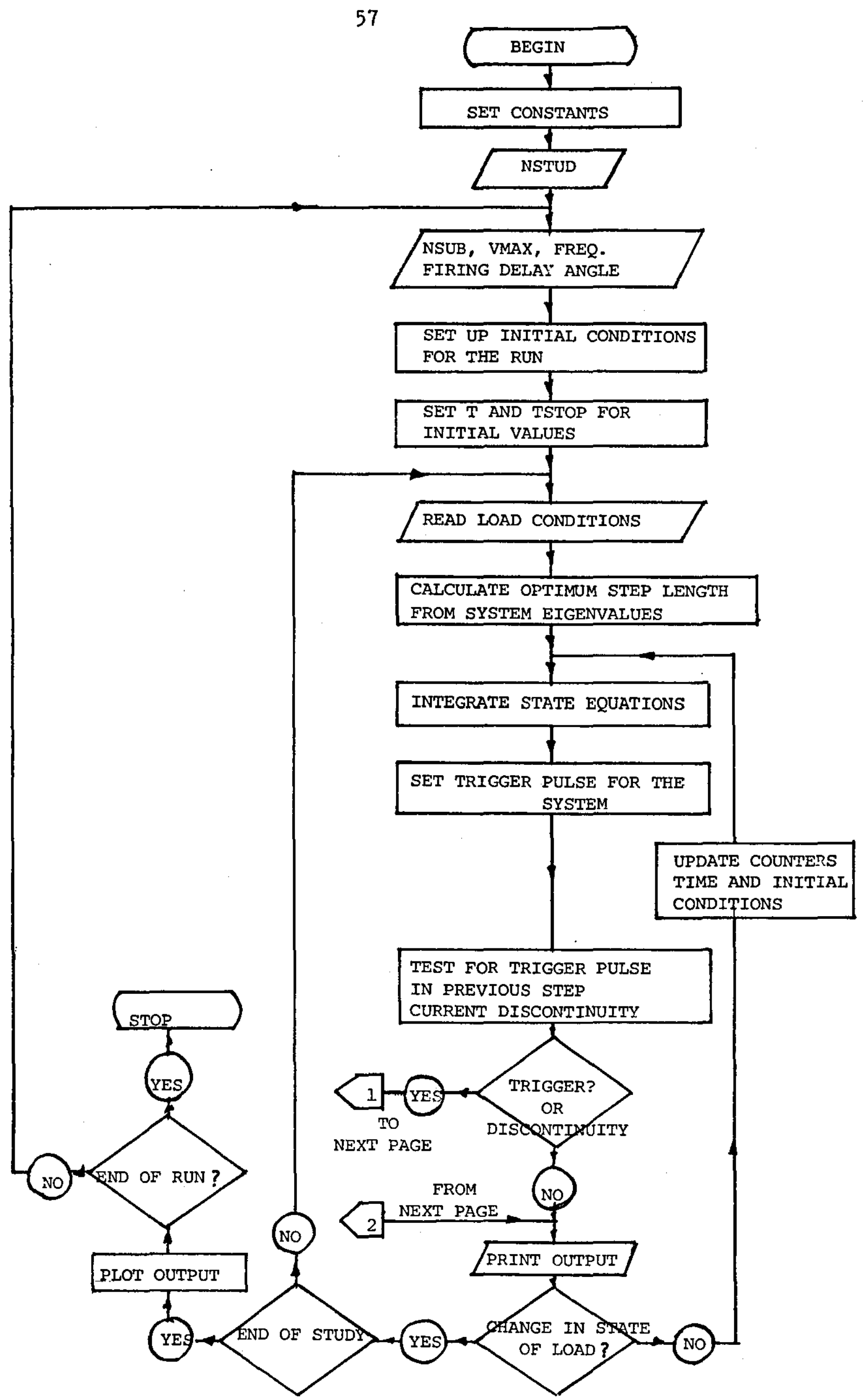

FIG. 4.1a SIMPLIFIED FLOWCHART FOR THE MAIN PROGRAM IN APPENDIX III 


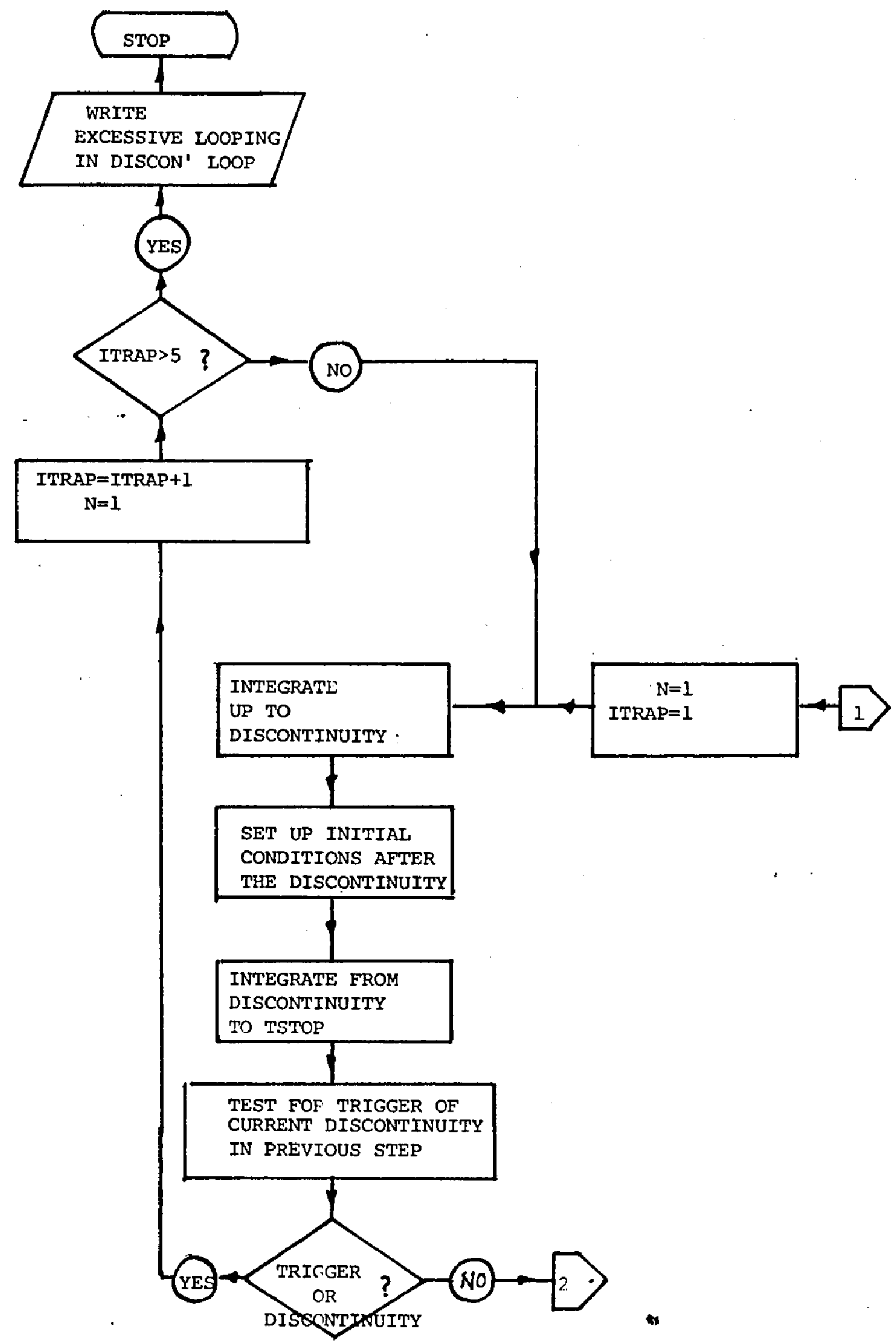

FIG. 4.la Simplified Flow-chart (Continuation from last page) 


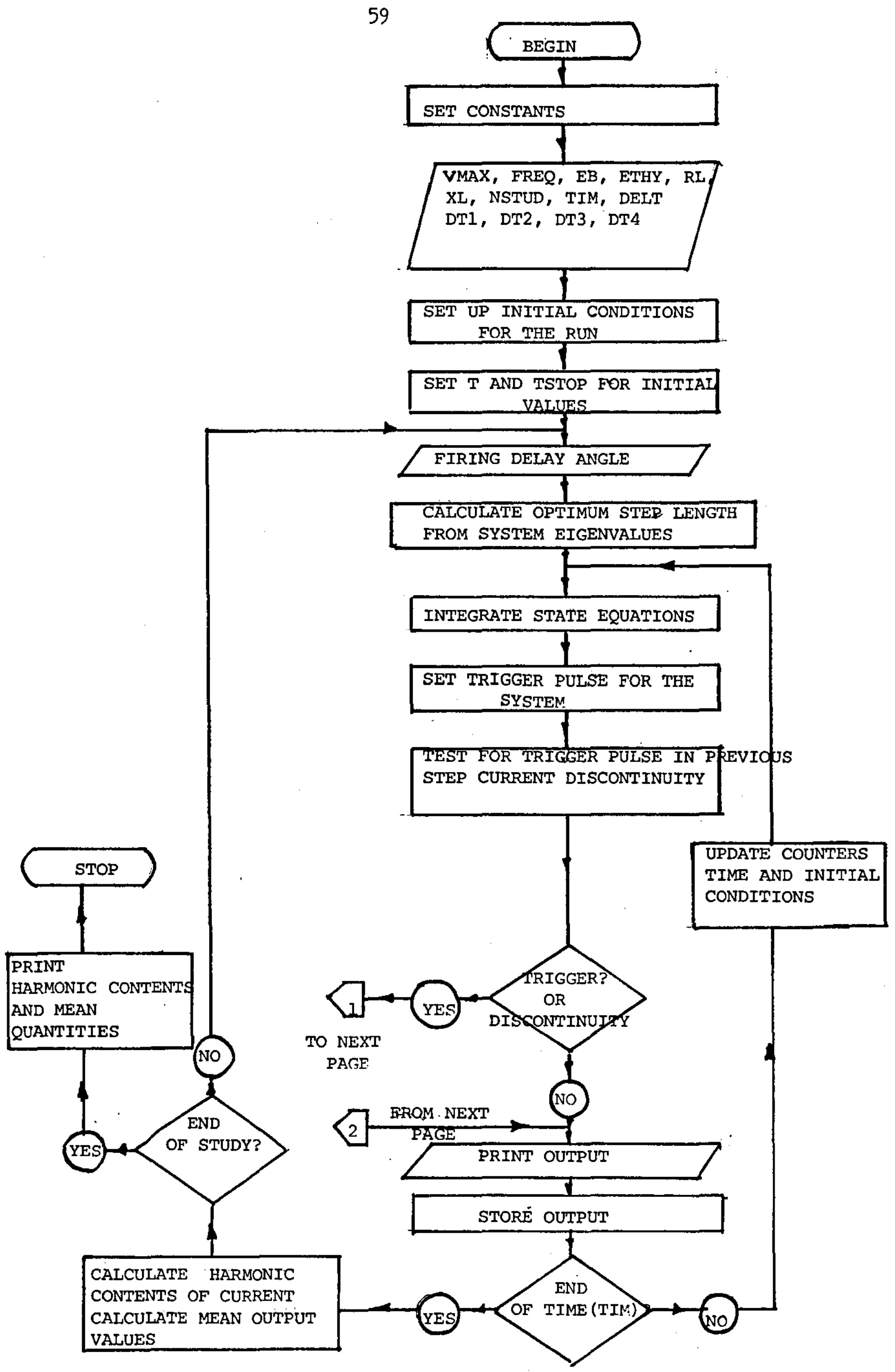

FIG. 4.1b Simplified Flowchart for the Main Program in Appendix IV 


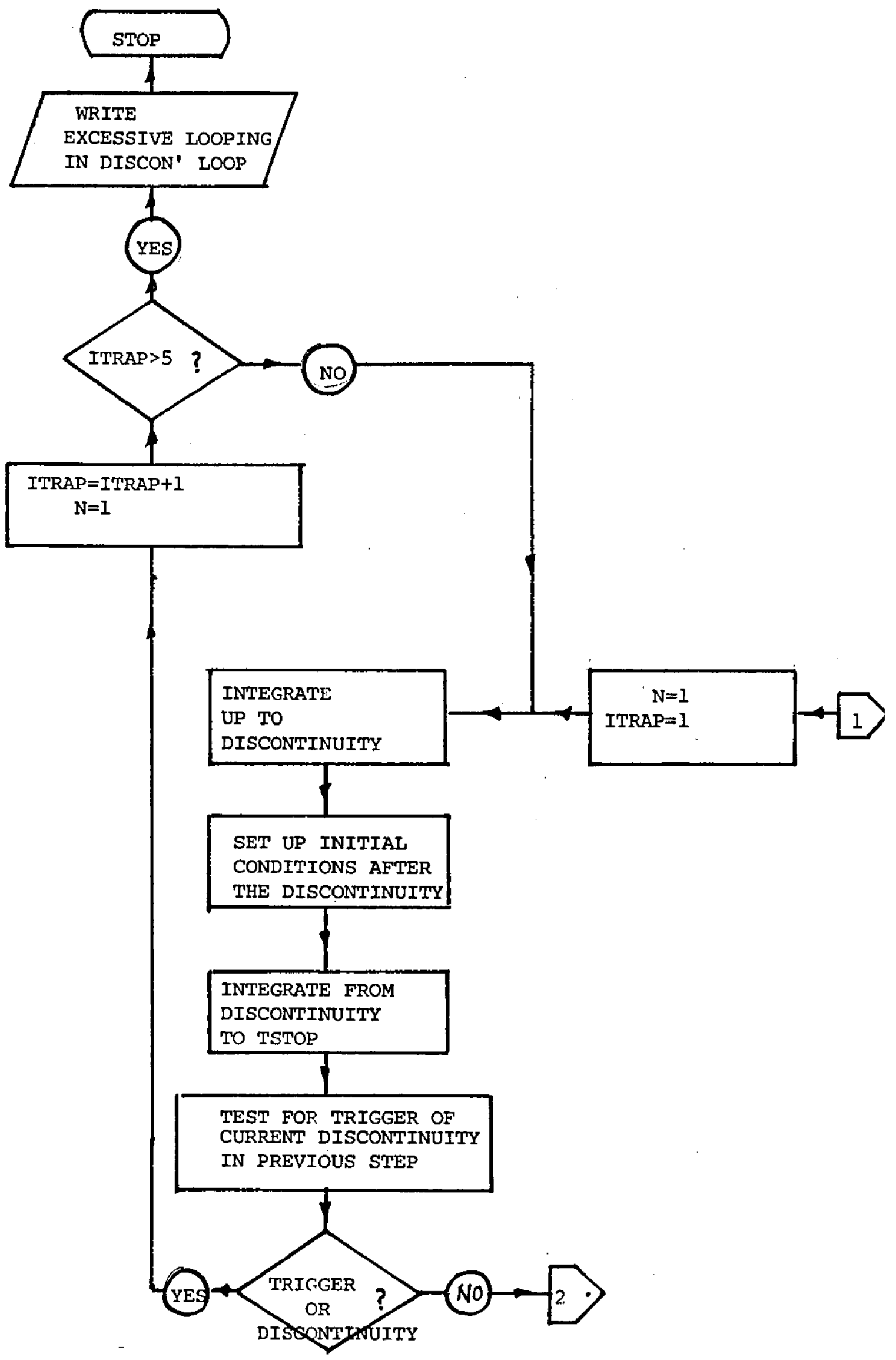

FIG. 4. 1p Simplified Flow-chart (Continuation from last page) 
case, to the meshes comprising devices $1 \mathrm{R}$ with $6 \mathrm{Y}$ and $1 \mathrm{R}$ ' with $6 \mathrm{Y}$ ' together with the fixed delta winding mesh. The elements of $\mathrm{CB}$ for this conduction pattern are shown in Fig. 4.2. Within the subroutine IOAD, the operation continues in order to implement the steps (a) and (b) of section 4.2. Also, the inverse of $L_{\mathrm{mm}}$ is obtained using subroutine MATINV.

Operations (c) to (e) of section 4.2 are implemented when subroutine $\mathrm{GN}$ is called successively as required by the integration subroutine MXRK4 and finally a solution to equation 4.1 is obtained. The values of $\mathrm{I}^{\mathrm{m}}$ are stored in the column vector $\mathrm{AN}$ in the program. Finally, operations (f) to (g) are carried out by subroutines BRANI and BRANV. In BRANI currents $I^{\dot{i}}$ and $I^{b}$ are stored in the arrays $A T$ and $A B$ respectively. In BRANV, $v_{b}$ is calculated and stored in the array VB. Next, topology changes are monitored using subroutine INTERP, whereby the program detects either a trigger pulse generated by subroutine TRIGER or a current discontinuity indicating thyristor turn-off. These processes are illustrated in the following sections.

\subsection{Trigger Pulse for the Thyristor Circuits}

The need to determine the instant of device turn-on and turn-off has been stated in the previous section. The former process is achieved by implementing the sequence of thyristor trigger pulses shown in Fig. 4.3. The implementation is achieved in the computer program such that each pulse fires a pair of thyristors to ensure that both conduct, e.g. $I R$ and $6 y$. Hence, two txigger pulses are shown in Fig. 4.3 for each device. This subroutine is called at the end of each step to check for the presence of the pulses in the operation of 
Fixed Variable

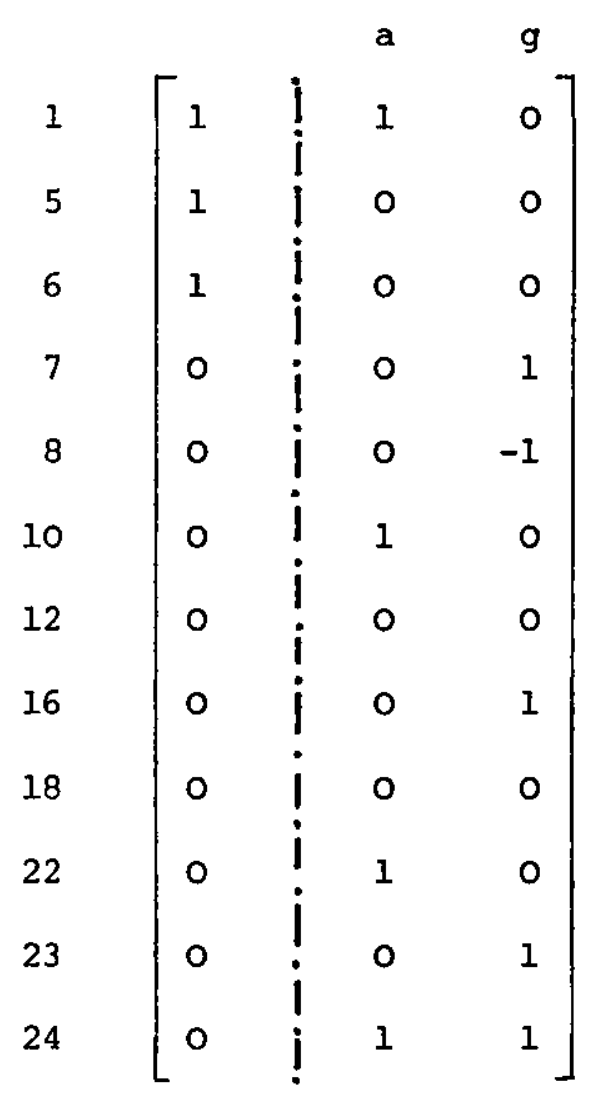

FIG. 4.2 MATRIX CB 


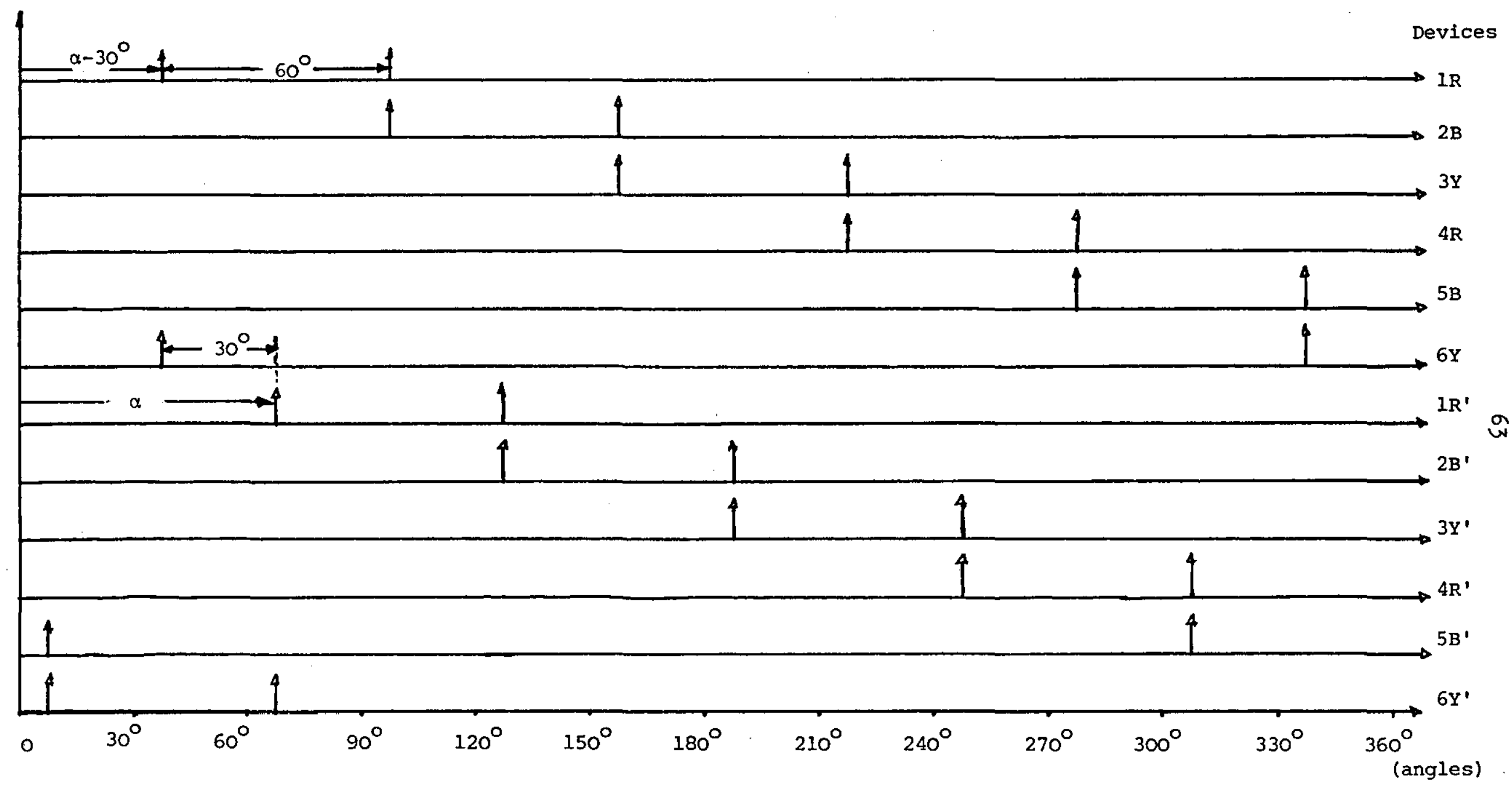

FIG. 4.3 Triggering Pulses for the Thyristors. Bridge I Operates $30^{\circ}$ in Advance of Bridge 2: 
the parallel bridge. A simplified flow chart of this subroutine is shown in Fig. 4.4 and it is called subroutine TRIGER in the computer program.

Apart from determining the presence of a trigger pulse the computer program also determines $t n$, the time of the occurrence of the pulse from the start of a step. This program is also designed to incorporate triggering unbalance that might possibly occur in practice between bridges and between the upper and lower rows of thyristors in each bridge.

\subsection{Current Discontinuity}

The mesh current is monitored to determine when a device ceases to conduct and this occurs when the current just becomes negative. Referring to Fig. 4.5 the time to the discontinuity, ta, is determined by,

$$
I_{11}+\frac{\left(I_{21}-I_{11}\right)}{t} \cdot \text { ta }=0
$$

from which

$$
t a=\frac{I_{11} t}{I_{11}-I_{21}}
$$

In the computer program, the initial currents at the start of a new run are set to a small value (10 $\mathrm{mA}$, rather than zero, to ensure that the program does not fail in the event of a current zero in the first step.

It is possible that one or more discontinuities (including the presence of a trigger pulsel are observed in a step. The times of these from the start of the step are determined as above and the shortest time is chosen for operation (i) to (vi) of section 4.2. 


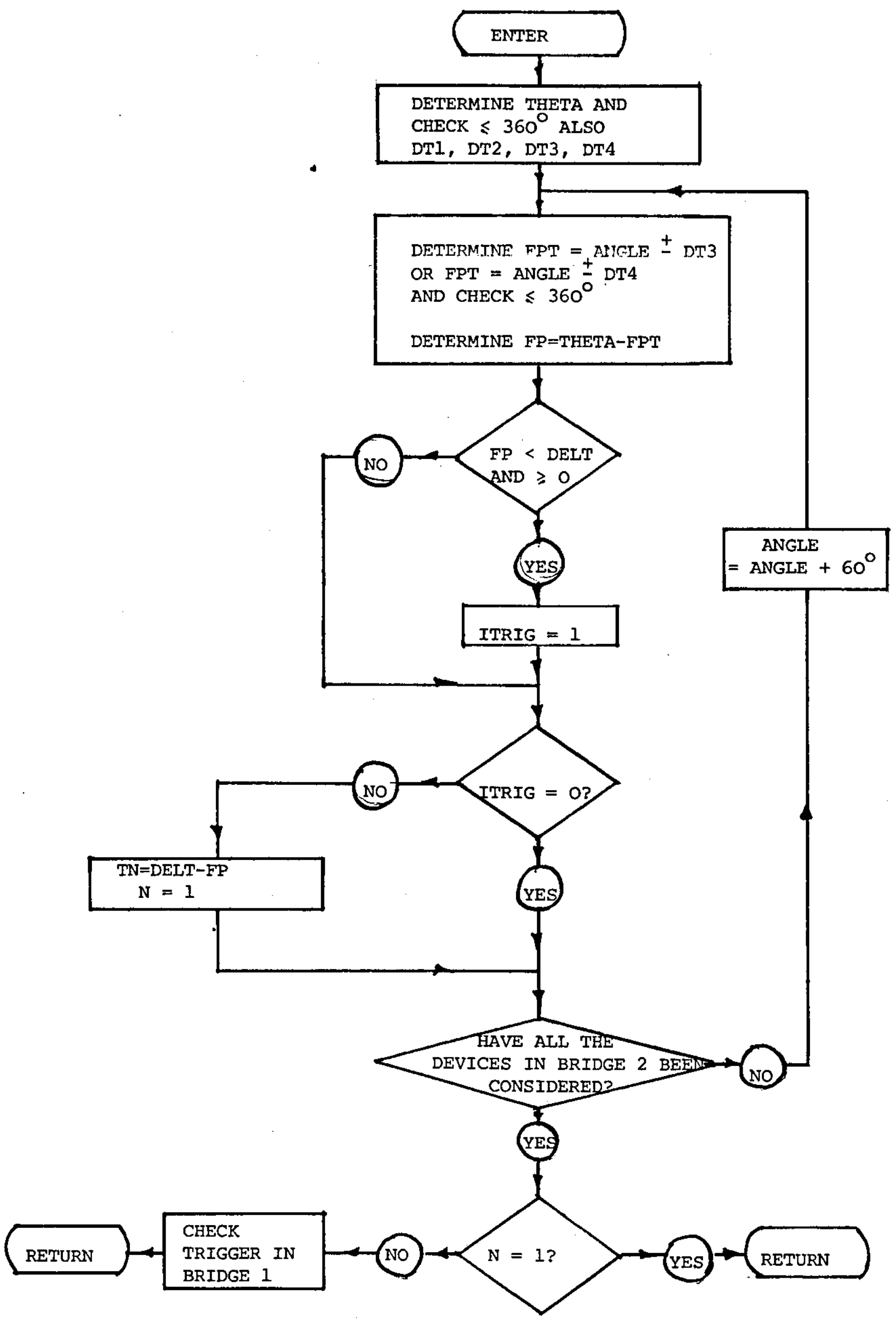

FIG. 4.4 Flowchart for Subroutine TRIGER (The Program is in Appendix IV) 


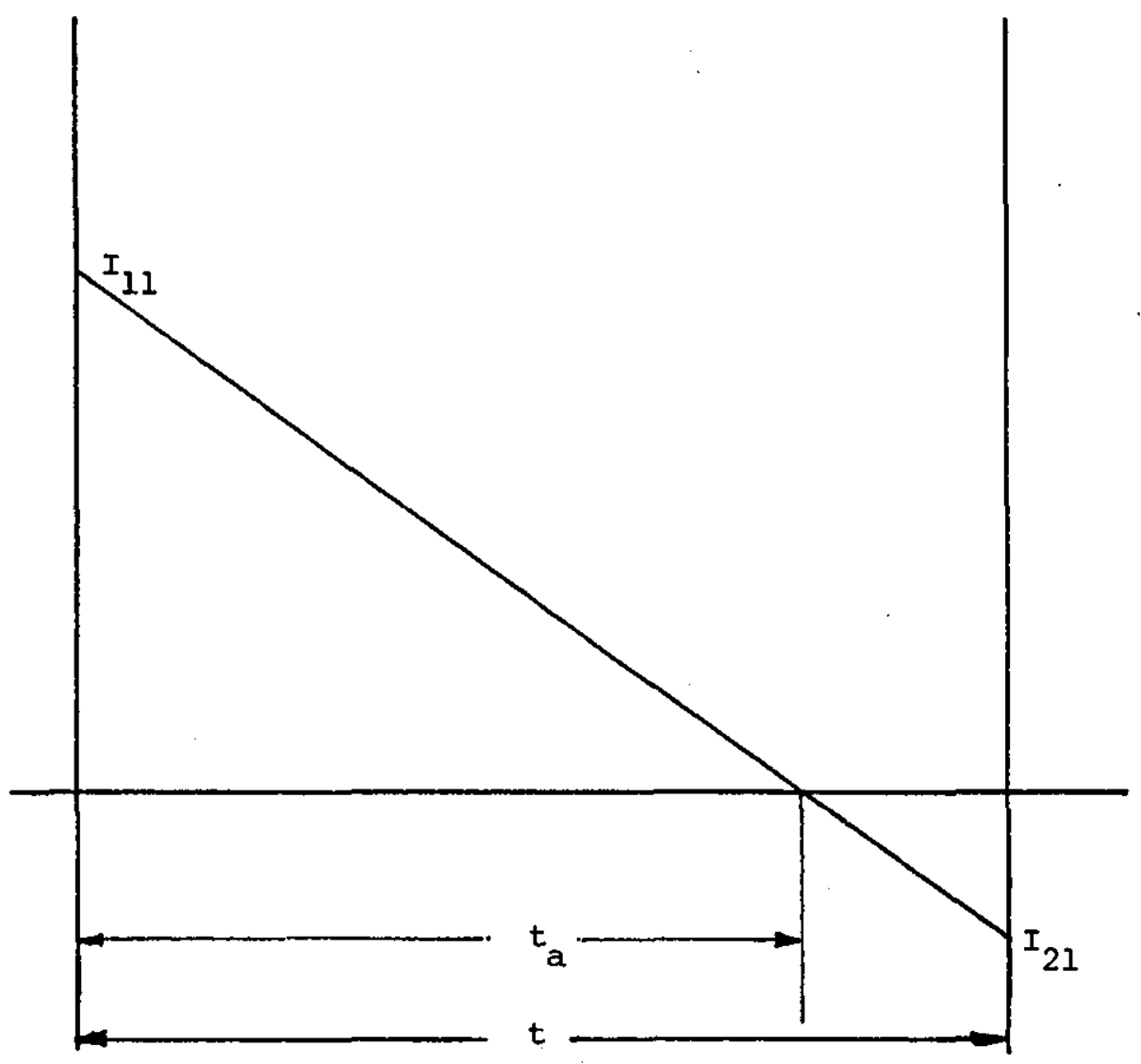

FIG. 4.5 Current Discontinuity Testing 


\subsection{Interpolation}

In the computer program the subroutine to perform the interpolation is called INTERP! The three main functions of this subroutine are;

(a) To check the forward biasing of the individual thyristors and the presence of a trigger pulse. The former is done separately for each bridge as the triggering of the thyristor pairs within the bridge are not at the same time. In the computer program, the thyristor anode-cathode voltage and the trigger pulse are denoted respectively by the arrays VD5 and ITRIG,

(b) To check if any currents, $I^{m}$ are negative. This is based on the calculation shown in section 4.5 ,

(c) Following any topology change due to either (a) or (b) above, the subroutine then makes the necessary changes to the mesh number $M$ and the loop patterns ICL.

The flowchart describing the operation performed by subroutine INTERP is illustrated in Fig. 4.6.

\subsection{Thyristor Voltage}

A thyristor has to be forward biased and a trigger pulse applied to its gate in order to conduct. This is achieved in the computer program using subroutine CDUCT5 which supplies the voltage drop for each individual thyristor. This subroutine determines the device voltages from the transformer branch voltages and the voltage drops due to device impedances during conduction. As an example, consider Fig. 4.7, which shows device $2 \mathrm{~B}^{\prime}$ as the next device to be triggered. Its voltage is determined from, 


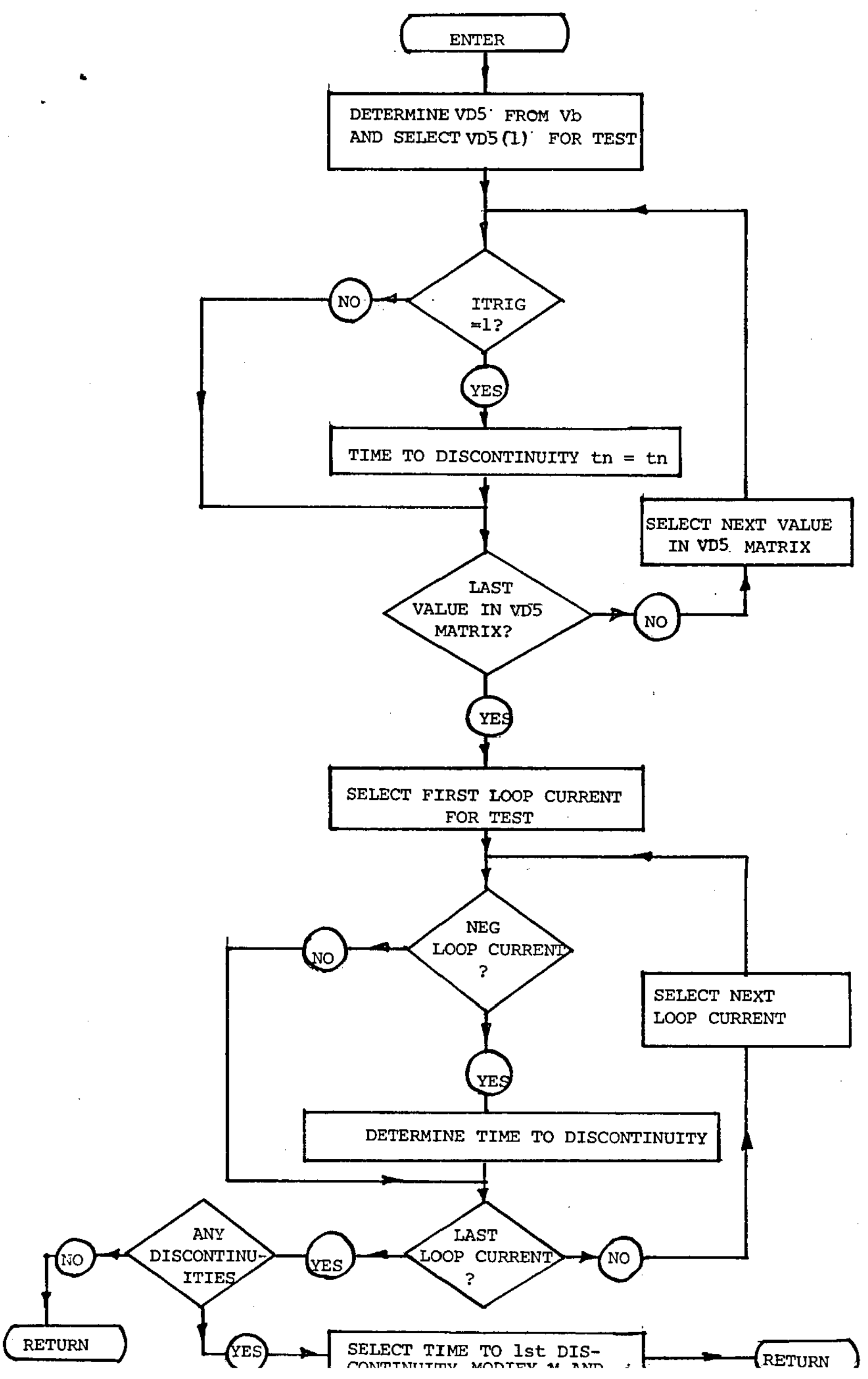




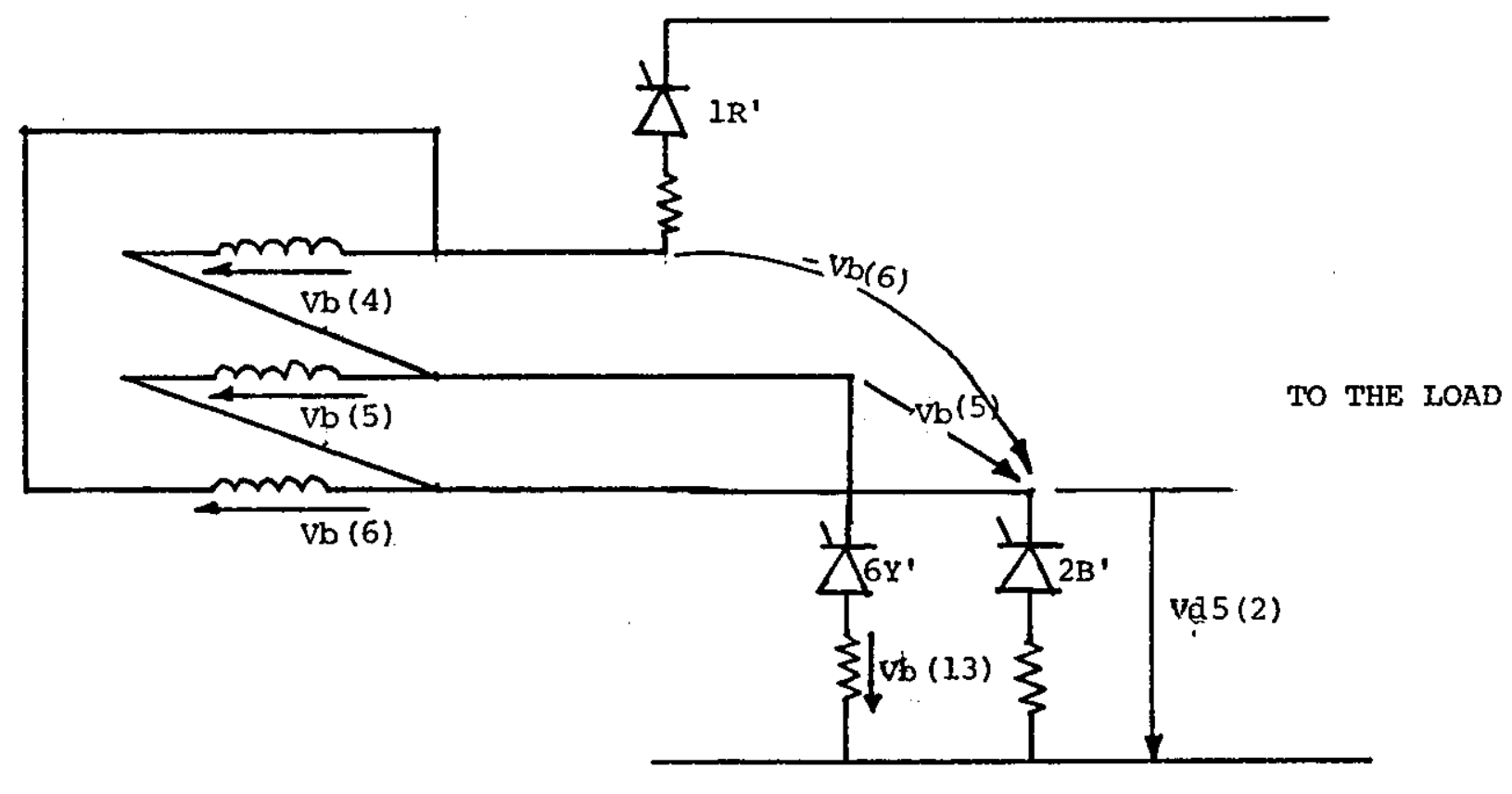

FIG. 4.7 Thyristor Voltage 


$$
\operatorname{Vas}(2)=\mathrm{Vb}(5)-\mathrm{Vb}(132
$$

similar expression defines other thyristor voltages and these

are implemented in the computer program.

The numbering in the bracket of the left-hand side of eqn. 4.2 refers to the thyristor numbers of Fig. 2.2 , where $1 R^{\prime}$ to $6 Y^{\prime}$ represent $I$ to 6 and $I R$ to $6 Y$ represent 7 to 12 , and on the righthand side refers to Fig. 3.8 .

Subroutine CDUCT is implemented in a similar fashion, but it is used for checking the thyristor voltage in the Mode 1 operation. This is because only a pair of thyristors is conducting at a time and the voltage is given by, $-\mathrm{Vb}(6)$ (see Fig.4.7) for thyristor pair $1 R^{\prime}$ and $2 B^{\prime}$ to conduct, thyristor $6 Y^{\prime}$ being switched off.

\subsection{Integration Procedure}

Techniques for numerically integrating differential equations may be classified into multi-step and single-step methods. Multistep methods, such as the various predictor/corrector formulae, are generally quicker and have greater stability than single-step methods. However, since they rely on integration ahead using open-type quadratic formulae (ref: 19) (in which the integration extends beyond the ordinates employed in the formulael, they require certain initial values to start the integration process. Clearly, such methods are precluded from thyristor circuit studies, since they would require re-starting at every system discontinuity. From the various singlestep methods available, a 4th-order Runge-Kutta routine has been chosen, due to its high accuracy. The Kutta-Merson method, which determines the truncation error at each step (at the expense of 
computer run-timel is unnecessary, since the maximum step length used by the computer program yields txuncation errors which are within error bounds.

The 4 th order Runge-Kutta equations are

$$
\begin{aligned}
G_{0} & =f\left(I_{t}, t\right) h \\
G_{1} & =f\left(I_{t}+\frac{1}{2} G_{0}, t+\frac{1}{2} h\right) h \\
G_{2} & =f\left(I_{t}+\frac{1}{2} G_{g}, t+\frac{1}{2} h\right) h \\
G_{3} & =f\left(I_{t}+G_{2}, t+h\right) h \\
I_{t+h} & =I_{t}+\frac{1}{6}\left(G_{0}+2 G_{1}+2 G_{2}+G_{3}\right)
\end{aligned}
$$

where $h$ is the integration step length; $f\left(I_{t}, t\right)$ etc. are the solutions of the mesh state variable equations for $I_{t^{\prime}} t$ etc; $I_{t}$ is the vector $I^{m}$ at time $t$, and $I_{t+h}$ is the vector $I^{m}$ at $t$ ime $(t+h)$.

A disadvantage of this integration method compared with other approaches is its relative instability. A procedure developed by williams (ref: 6) for selecting the optimum step length with respect to numerical stability and is outlined below.

\subsection{Optimum Step Length}

The optimum step length for a Runge-Kutta integration procedure is equal to the shortest system time constant. The system time constants can be obtained by determining the elgenvalues of the characteristic equation formed from the state matrix of the state variable equation. That is, from the standard state-variable equation

$$
[\dot{\mathrm{x}}]=[\mathrm{A}][\mathrm{X}]+[\mathrm{B}][\mathrm{U}] \text {, }
$$

the eigenvalues of $[\mathrm{A}]$ are found. These appear in the solution 
$e^{-\lambda_{n} t}$ terms, where $\lambda_{n}$ is the eigenvalue of the $n^{t h}$ local solution. The largest efgervalue is the reciprocal of the smallest time constant (ref: 12,13 ).

The computer program determines the shortest time constant using a NAG library subroutine, and sets the step length accordingly. This subroutine is called OPSTEP in the computer program. However, it may be removed if not required since after several runs, a step length of 0.0001 seconds is justified for the computation.

\subsection{Input Data}

Two computer program listings are presented in Appendices III andIV. These are used specifically to obtain respectively the current waveforms and their harmonic contents.

This is a convenient approach and reduces computing time from that needed when both functions are incorporated in a single program. It follows from this that two input data are needed as shown at the end of the listings. The first will be explained here and the other in Chapter 5.

In Appendix III, there are nine data lines read by the computer successively as follows:

Line 1. Three transformer winding phase resistances and the sixth number is the thyristor resistance.

Lines 2 to 4. Transformer self and mutual inductances with the last number being the thyristor path self inductance if any.

Line 5. NSTUD - number of studies to be made.

Line 6. Peak supply line voltage, frequency and fixing delay angle. Line 7. NSUB - number of sample. 
Line 8. Time to complete the run per sample (seconds)

Line 9. Load data; R, L, Ed and e thy 


\section{CHAPTER 5 HARMONIC ANALYSIS AND MEAN QUANTITIES}

\subsection{Introduction}

The current waveforms of the model are fuxther analysed for their harmonic contents using Fourier Analysis. This section briefly outlines the Fourier Analysis routine. In aiding the discussion, a simplified flow chart is given in Fig. 5.1. Also, the mean output currents and voltage of the parallel converter circuit will be discussed with respect to their computer implementation.

\subsection{Fourier Analysis}

Generally speaking, any periodic signal $f(t)$ may easily be analysed if it may be expressed in terms of sinusoidal signals. That is, the signal is said to be expressed in terms of its Fourier series as shown below;

$$
\begin{aligned}
f(t)= & \frac{a_{0}}{2}+a_{1} \cos \omega_{0} t+b_{1} \sin \omega_{0} t+a_{2} \cos 2 \omega_{0} t \\
& +b_{2} \sin 2 \omega_{0} t+\ldots \ldots \\
\text { or } f(t)= & \frac{a_{0}}{2}+\sum_{n=1}^{\infty} a_{n} \cos n \omega_{0} t+\sum_{n=1}^{\infty} b_{n} \sin n \omega_{0} t
\end{aligned}
$$

For any specific signal, $f(t)$, as shown above. Fourier Analysis can be carried out. This involves calculating the coefficient $a_{\circ}$ $a_{n}$ and $b_{n}$ for the given signal (ref: 20,21 ).

The series of equation 5.I may be written in a number of apparently different, although equivalent forms, one of which is obtained by recognising that for all values of $n$,

$$
\begin{aligned}
& a_{n} \cos n \omega_{0} t+b_{n} \sin n \omega_{0} t \\
& =c_{n} \sin \left(n \omega_{0} t+\theta_{n}\right)
\end{aligned}
$$




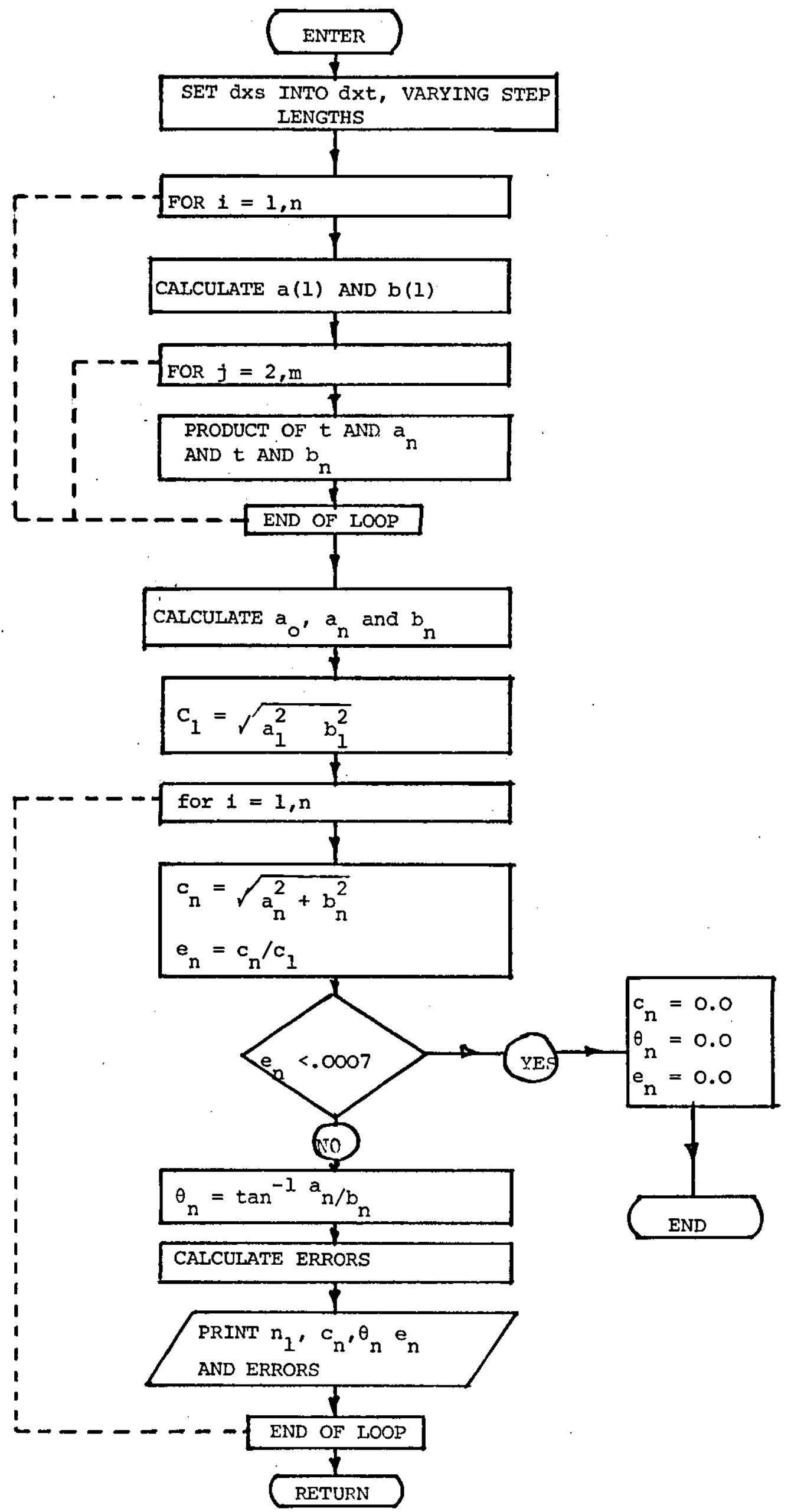

FIG. 5.1 FLOIRCHAPT FOR SUBROUTINE FOURER 


$$
\text { where, } \begin{aligned}
c_{n} & =\sqrt{a_{n}^{2}+b_{n}^{2}} \\
\sin \theta_{n} & =\frac{a_{n}}{\sqrt{a_{n}^{2}+b_{n}^{2}}} \\
\cos \theta_{n} & =\frac{b_{n}}{\sqrt{a_{n}^{2}+b_{n}^{2}}}
\end{aligned}
$$

and hence

$$
\theta_{n}=\tan ^{-1}\left(a_{n} / b_{n}\right)
$$

Combination pairs of terms in equation 5.2 gives the equivalent form for the Fourier Series, (ref: 22).

$$
f(t)=c_{0}+c_{1} \sin \left(\omega_{0} r+\theta_{1}\right)+\ldots c_{n} \sin \left(n \omega_{0} t+\theta_{n}\right)
$$

with $c_{0}$ equal to $a_{0} / 2$ and all other $c_{n}$ and $\theta_{n}$ defined by equations 5.3 to 5.6. The coefficient $c_{n}$ is the amplitude and $\theta_{n}$, the phase of the $n^{\text {th }}$ harmonic.

The Fourier Analysis of such a signal $f(t)$, is widely covered in the literature. The numerical estimation of the Fourier coefficients are obtained as follows:

(a) Estimation of a

$$
\frac{a_{0}}{2}=\frac{\sum_{i=0}^{m} f\left(t_{i}\right)}{m}
$$

(b) Estimation of $a_{n-}$

$$
a_{n}=\frac{2 \sum_{i=1}^{m} f\left(t_{i}\right) \cos n \omega_{o} t_{i}}{m}
$$

(c) Estimation of $b_{n-}$

$$
b_{n}=\frac{2 \sum_{i=1}^{m} f\left(t_{i}\right) \sin n \omega_{0} t_{i}}{m}
$$

From these numerical estimates for $a_{n}$ and $b_{n}$, the amplitude $c_{n}$ and 
phase $\theta_{n}$ of harmonics can be readily calculated using the digital computer.

\subsection{Variation of the step length in the estimation of the Fourier Coefficients.}

Up to this point, the numerical estimation of the Fourier Series has assumed a constant step length. Sometimes, the signal $f(t)$ under study, particularly the current or voltage waveforms, exhibit discontinuity. For improvement of the estimate, any discontinuities in the signal have to be determined exactly and this necessitates using new intermediate steps within the step where the discontinuity occurs.

Consider Fig. 5.2 where $y=f(t)$ is known for $m$ values. of $t$. These are designated as $\mathrm{y}_{0}, \mathrm{Y}_{1}, \mathrm{y}_{2}, \mathrm{y}_{3} \ldots \mathrm{y}_{\mathrm{m}}$. The contribution of the first interval, shown as abcd in Fig. 5.2 is approximately,

$$
\frac{1}{2} \frac{\left(y_{0}+y_{1}\right)}{\Delta t}
$$

This is the area of a trapezoid having an average amplitude of $\left(y_{0}+y_{1} l / 2\right.$ and a base equal to $\Delta t$. Similarly, the contribution of the second interval is,

$$
\frac{1}{2} \frac{\left(y_{1}+y_{2}\right)}{\Delta t}
$$

It can be seen that in forming the summation of the areas all values of $y$ are used twice. Hence, for the case of equal step lengths (i.e. $\Delta t$ is equal throughoutl, this gives rise to equation 5.8. Equations 5.9 and 5.10 are similarly solved by this approach (ref: 21 ). Suppose a discontinuity occurs in the signal $f(t)$ of Fig. 5.2 


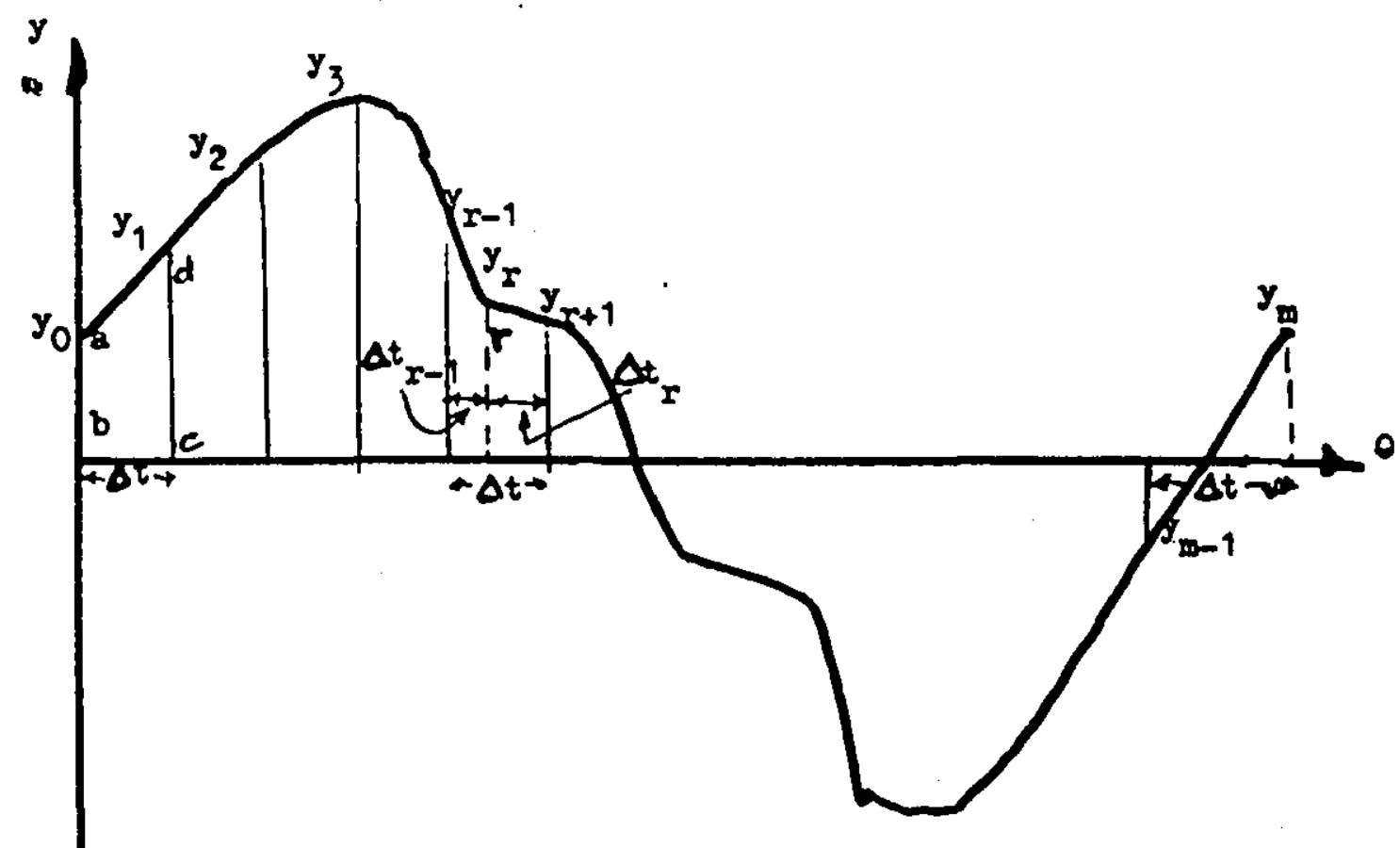

FIG. 5.2 SIGNAL $f(t)$ INDICATING THE OCCURRENCE OF DISCONTINUITY

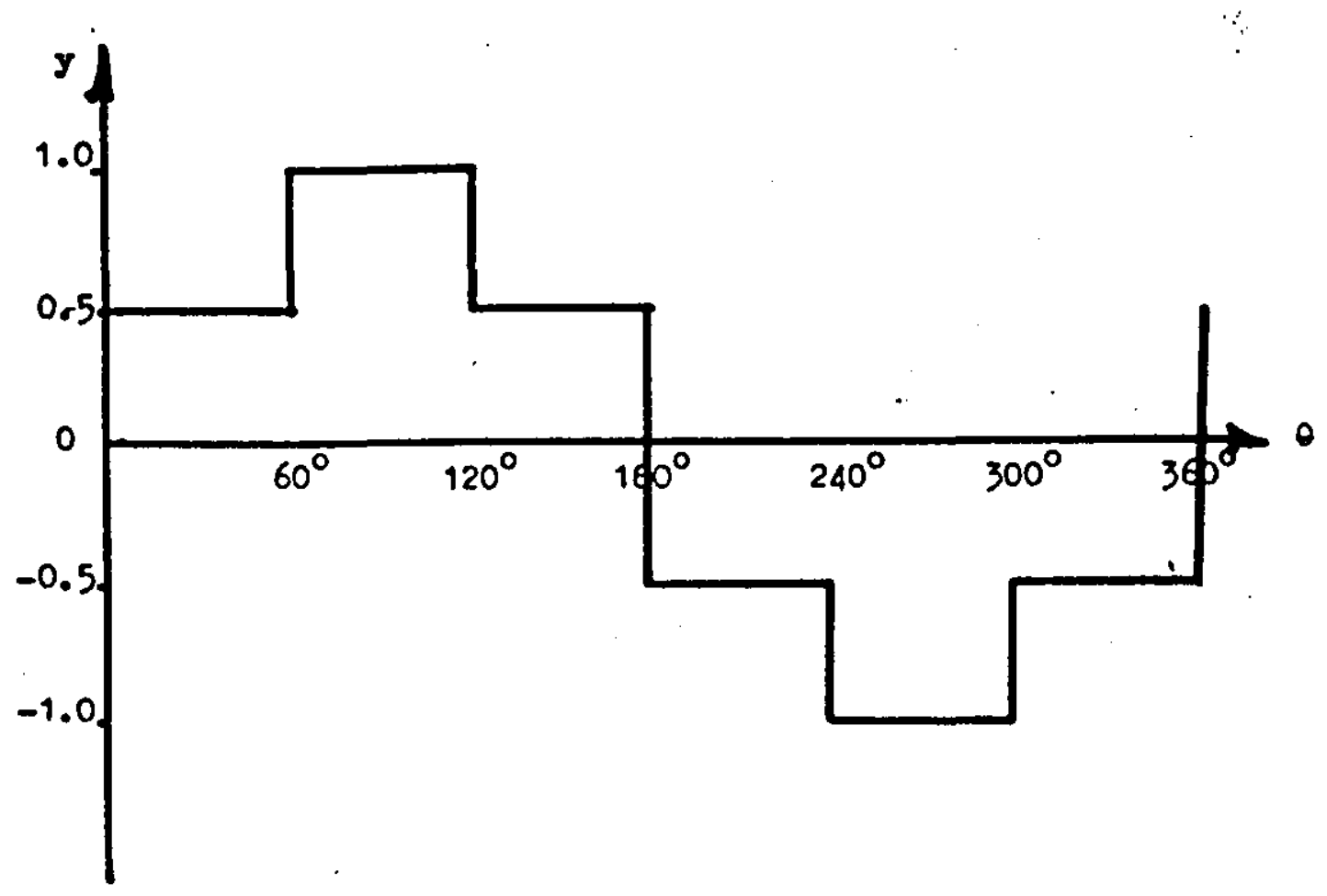

FIG. 5.3 WAVEFORM GENERATED BY THE COMPUTER FOR THE TEST 
at point $r$ within a step length $\Delta t$. This discontinuity has to be determined exactly, which eventually gives rise to two new independent step lengths, $\Delta t_{r}$ and $\Delta t_{r-1}$. With the introduction of these new step lengths, equations $5.8,5.9$ and 5.10 are modified accordingly as shown below:

(a) Estimation of a

$\frac{a_{0}}{2}=\frac{1}{m}\left[\frac{y_{0}+y_{1}}{2 \Delta t}+\frac{y_{1}+y_{2}}{2 \Delta t}+\ldots \frac{y_{r-1}+y_{r}}{2 \Delta t_{r-1}}+\frac{y_{r}+y_{r+1}}{2 \Delta t_{r}}+\ldots \frac{y_{m-1}+y_{m}}{2 \Delta t}\right]$

(b) Estimation of $\mathrm{a}_{\mathrm{n}}$

$a_{n}=\frac{1}{m}\left[\frac{y_{0} \cos n \omega_{0} t_{0}+y_{1} \cos n \omega_{0} t_{1}}{\Delta t}+\frac{y_{1} \cos n \omega_{0} t_{1}+y_{2} \cos n \omega_{0} t_{2}}{\Delta t}\right.$

$$
\begin{aligned}
& +\ldots \frac{y_{r-1} \cos n \omega_{0} t_{r-1}+y_{r} \cos n \omega_{o} t_{r}}{\Delta t_{r+1}} \ldots+\frac{y_{r} \cos n \omega_{o} t_{r}+y_{r+1} \cos n \omega_{o} t_{r+1}}{\Delta t_{r}} \\
& \left.\ldots+\frac{y_{m-1} \cos n \omega_{o} t_{m-1}+y_{m} \cos n \omega_{o} t_{m}}{\Delta t}\right]
\end{aligned}
$$

(c) Estimation of $b_{n-1}$

$b_{n}=\frac{1}{m}\left[\frac{y_{0} \sin n \omega_{0} t_{0}+y_{1} \sin n \omega_{0} t_{1}}{\Delta t}+\frac{y_{1} \sin n \omega_{0} t_{1}+y_{2} \sin n \omega_{0} t_{2}}{\Delta t}\right.$

$$
\begin{aligned}
& +\ldots \frac{y_{r-1} \sin n \omega_{0} t_{r-1}+y_{r} \sin n \omega_{o} t_{r}}{\Delta t_{r+1}} \\
& +\frac{y_{r} \sin n \omega_{0} t_{r}+t_{r+1} \sin n \omega_{0} t_{r+1}}{\Delta t_{r}} \\
& \left.\ldots+\frac{y_{m-1} \sin n \omega_{0} t_{m-1}+y_{m} \sin n \omega_{0} t_{m}}{\Delta t}\right]
\end{aligned}
$$

For the estimation of $a_{n}$ and $b_{n}$, the factor 2 which appeared in equation 5.9 and 5.10 is missing above. This factor appears in the expressions for the areas of the trapezoids of Fig. 5.2 and cancels. 


\subsection{Computer estimation of the Fourier Series for a known signal}

Equation $5.13,5.14$ and 5.15 wexe implemented in a computex program. Tests on the effectiveness of this program to estimate the Fourier Series were carried out by, firstly, generating a knowin signal as shown in Fig. 5.3. The inputted numerical values for. the waveform include the exact points of the occurrence of the discontinuities at $\theta=60^{\circ}, 120^{\circ}, 180^{\circ}$, etc. For this particular waveform at the discontinuity the step length is zero.

The results for this test are depicted in Table 5.1 using 200 sample points. The table also provides the errors for each harmonic component as compared with the values obtained by exact trigonometric calculation. The errors are reduced by increasing the sample points.

A second test involves supplying the coefficients $C_{n}$ and $\theta_{n}$ in the equation 5.7 to generate a waveform. This was then analysed to obtain the corresponding values of $c_{n}$ and $\theta_{n^{*}}$ similarly this was done with about 200 sample points. Table 5.2 provides the results.

The results of both tests are depicted in Tables 5.1 and 5.2 and these indicate the ability of the computer program to analyse any periodic signal.

\subsection{Mean dc output quantities}

The dc bridge currents and output voltage are obtained using a simple trapezoidal algorithm. A flowchart is depicted in Fig. 5.4 and this is named subroutine RMSAVE in the computer program of AppendixIV. Also, the program can evaluate the rms value of a given function. 


\begin{tabular}{|c|c|c|c|c|c|}
\hline $\begin{array}{c}\text { harmonic no: } \\
0.0 \\
1.0 \\
2.0 \\
3.0 \\
4.0 \\
5.0 \\
6.0 \\
7.0 \\
8.0 \\
9.0 \\
10.0 \\
11.0 \\
13.0 \\
13.0 \\
14.0 \\
15.0 \\
19.0 \\
17.0 \\
18.0 \\
19.0 \\
20.0\end{array}$ & $\begin{array}{c}m 01 \\
0.9548530+00 \\
0.0000000+00 \\
0.0000000+00 \\
0.0000000+00 \\
0.9905570+00 \\
0.0000000+00 \\
0.1359420+00 \\
0.0000000+00 \\
0.0000000+00 \\
0.0000000+00 \\
0.8577480-01 \\
0.0000000+00 \\
0.7269170-01 \\
0.0000000+00 \\
0.0000000+00 . \\
0.0000000+03 \\
0.5443320-01 \\
0.0000000+00 \\
0.4931950-01 \\
0.0000000+00\end{array}$ & $\begin{array}{r}2 r g \\
90.00 \\
0.00 \\
0.00 \\
0.00 \\
90.00 \\
0.00 \\
270.00 \\
0.00 \\
0.00 \\
0.00 \\
270.00 \\
0.00 \\
90.00 \\
0.00 \\
0.00 \\
0.00 \\
90.00 \\
0.00 \\
270.00 \\
0.00\end{array}$ & $\begin{array}{c}\text { D.u of fund. } \\
0.0000000 \\
1.0000000 \\
0.0000000 \\
0.0000000 \\
0.0000000 \\
0.1995668 \\
0.0000000 \\
0.1423678 \\
0.0000000 \\
0.0000000 \\
0.0000000 \\
0.0898304 \\
0.0001000 \\
0.0761287 \\
0.0000000 \\
0.0000000 \\
0.0000000 \\
0.0570069 \\
0.0000000 \\
0.0516514 \\
0.0100000\end{array}$ & $\begin{array}{r}\text { derivation } \\
1.0000000 \\
0.0000000 \\
0.0000000 \\
0.0000000 \\
0.2000000 \\
0.0000000 \\
-0.1428571 \\
0.0000000 \\
-0.0000000 \\
0.0040000 \\
-0.0909091 \\
0.0000000 \\
0.0769231 \\
0.0000000 \\
0.0000000 \\
0.0000000 \\
0.0585235 \\
0.0000000 \\
-0.0526316 \\
0.0000000\end{array}$ & $\begin{array}{l}\text { xerror } \\
0.0000 \\
0.0000 \\
0.0000 \\
0.0000 \\
0.2166 \\
0.0000 \\
0.3611 \\
0.0000 \\
0.0000 \\
0.0000 \\
1.1855 \\
0.0000 \\
1.0327 \\
0.0000 \\
0.0000 \\
0.0000 \\
3.0883 \\
0.0000 \\
1.8623 \\
0.0000\end{array}$ \\
\hline
\end{tabular}




\begin{tabular}{|c|c|c|c|c|c|}
\hline $\begin{array}{c}\text { harmonic no: } \\
0.0 \\
1.0 \\
2.0 \\
3.0 \\
4.0 \\
5.0 \\
9.0 \\
7.0 \\
9.0 \\
9.9 \\
10.0 \\
19.0 \\
12.9 \\
13.1 \\
14.0 \\
15.0 \\
16.0 \\
17.0 \\
19.9 \\
19.0 \\
20.0\end{array}$ & $\begin{array}{c}m 0 d \\
0.9549100+00 \\
J .0000000+00 \\
0.0000000+00 \\
0.0000000+00 \\
0.1908800+00 \\
0.0000000+00 \\
0.1362900+00 \\
0.0009000+00 \\
0.000000 D+00 \\
0.0000000+00 \\
0.8656990-01 \\
0.0000000+00 \\
0.7323010-01 \\
3.0000000+00 \\
0.0000000+03 \\
0.0000000+00 \\
0.5579010-01 \\
0.0000000+00 \\
0.4996990-01 \\
0.0000000+00\end{array}$ & $\begin{array}{r}\text { ara } \\
\\
90.00 \\
0.00 \\
0.00 \\
0.00 \\
90.00 \\
0.00 \\
270.00 \\
0.00 \\
0.00 \\
0.00 \\
270.00 \\
0.00 \\
90.00 \\
0.00 \\
0.00 \\
0.00 \\
90.00 \\
0.00 \\
270.00 \\
0.00\end{array}$ & $\begin{array}{c}0 . u \text { of fund. } \\
0.0000000 \\
1.0000000 \\
0.0000000 \\
0.0000000 \\
0.0000000 \\
0.1998933 \\
0.0000000 \\
0.1427254 \\
0.0000000 \\
0.0000000 \\
0.0000000 \\
0.0906576 \\
0.0000000 \\
0.0766880 \\
0.0000000 \\
0.0000000 \\
0.0000000 \\
0.0584264 \\
0.0000000 \\
0.0523085 \\
0.0000000\end{array}$ & $\begin{array}{l}\text { derivation } \\
1.0000000 \\
0.0000000 \\
0.0000000 \\
0.0000000 \\
0.1998932 \\
0.0000000 \\
0.1627255 \\
0.0000000 \\
0.0000000 \\
0.0000000 \\
0.0906578 \\
0.0000000 \\
0.0766879 \\
0.0000000 \\
0.0000000 \\
0.0000000 \\
0.0586244 \\
0.0000000 \\
0.0523086 \\
0.0000000\end{array}$ & $\begin{array}{l}\text { zerror } \\
0.0000 \\
0.0000 \\
0.0000 \\
0.0000 \\
0.0000 \\
0.0000 \\
0.0001 \\
0.0000 \\
0.0000 \\
0.0000 \\
0.0001 \\
0.0000 \\
0.0001 \\
0.0000 \\
0.0000 \\
0.0000 \\
0.0002 \\
0.0000 \\
0.0002 \\
0.0000\end{array}$ \\
\hline
\end{tabular}




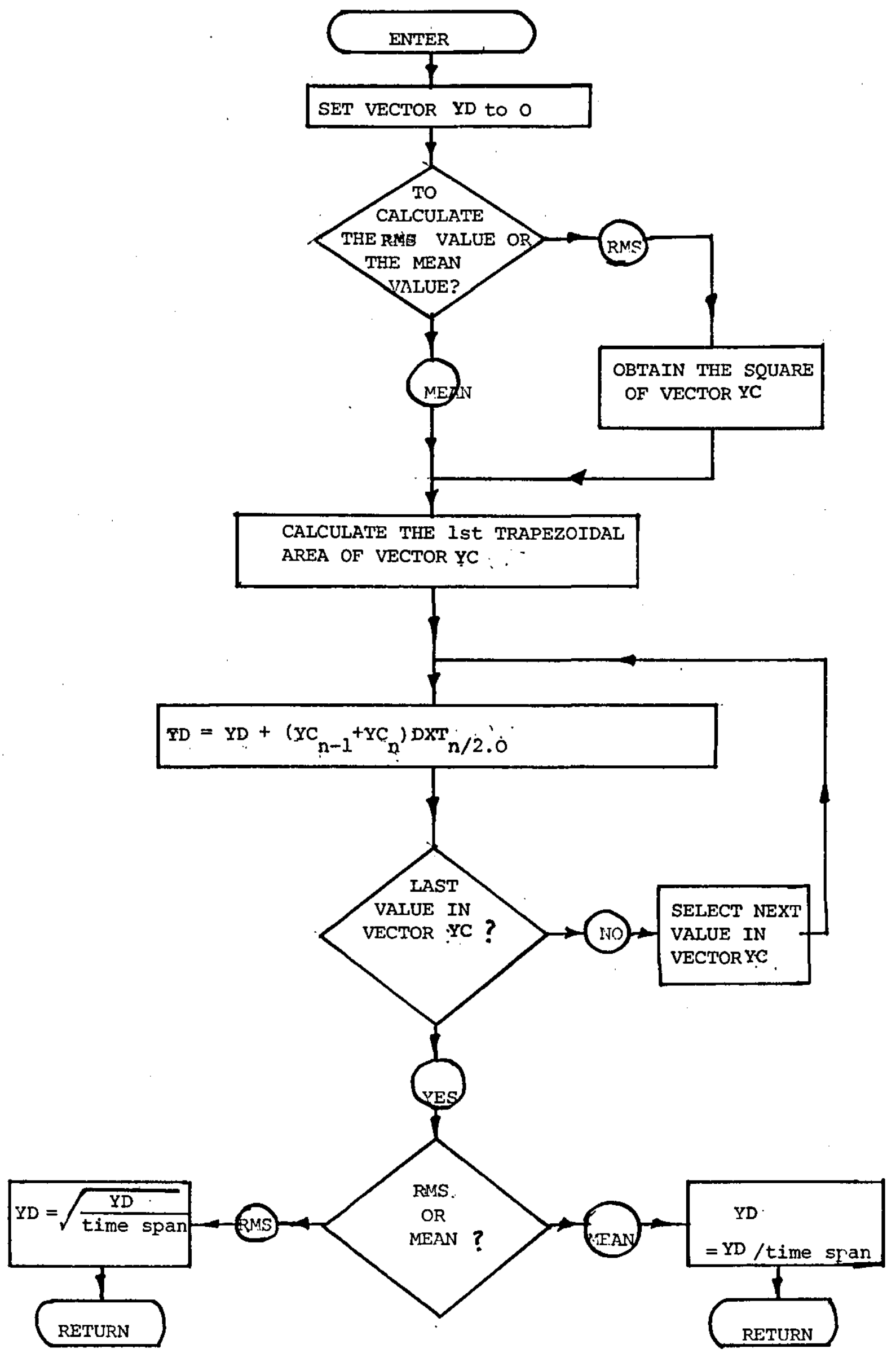

FIG. 5.4 Flow chart for the Subroutine RMSAVE 


\subsection{Input data}

A separate input data is necessary for the program and this is explained here.

The input data as printed at the end of the Appendix IV has four lines to be read by the computer. They are as follows:

Line 1. Peak supply line voltage; frequency; load back emf; thyristor voltage drop; load resistance and inductance. Line 2 NSTUD, time, step length.

Line 3 triggering variation for the thyristors in the top and bottom part of each bridge and between the bridges. The first two numbers pertain to bridge 1 while the last two pertain to bridge 2 .

Line 4 Firing delay angles; a number of firing delay angles can be set after this line depending upon the value of NSTUD (in line 2). For example, to study one case of tiring delay angle, NSTUD is set to 1. 


\subsection{Introduction}

The purpose of this chapter is to present the results which;

a) validate the computer program by comparing the computed and the experimental results and;

b) demonstrate the operational characteristics of the parallelbridge system considered.

A description of the experimental laboratory arrangement is presented to provide an insight to its features. However, its constructional details are omitted for conciseness.

It is important that the impedances and voltages are well balanced between the two secondary windings of each phase, and between the three phases. Special attention is focussed on the adjustments made to the transformers to ensure acceptable balance.

The computer program of the developed mathematical model presented in Chapter 3 can be executed when typical circuit parameters are available. These parameters comprise the transformer data, the thyristor modelling data and the load data together with operational details such as supply voltage and firing delay angle. The measurements of these data from the laboratory convertor system will be outlined. Validation of the computer program is achieved by comparison of the computed and measured current waveforms in the transformer windings and ac supply lines. The capability of predicting these waveforms is, of course, one of the objectives of the research.

Finally, the various operating characteristics plotted from the computer program are presented and discussed. Some of these, as appropriate, are verified experimentally. 


\subsection{The Laboratory Convertor System}

Photographs of the 12-pulse parallel convertor system used are shown in Fig. 6.1 with the pulse shifting transformer and its protractor in evidence. The supply transformers are shown in Fig. 6.2 with the associated extra bucking and boosting transformers and inductances needed to improve balance. The circuit connections are shown schematically in Fig. 6.3.

As stated earlier, three separate single-phase, three winding transformers are used to supply the bridge rectifiers, these having been adapted from existing two-winding transformers with split secondary windings. Not being purpose-built for the role, improvisation has been necessary to provide the required voltage and impedance balance previously mentioned. For equal and balanced supply voltage to the two bridges, additional bucking transformers are used with the starsecondary and boosting transformers with the delta secondary windings (Fig. 6.3L. Extra air-cored reactors are included in each transformer phase to give equivalent circuit inductance balance between the two secondary windings in each phase, and between the three phases. This procedure involved repeated short circuit testing and adjustment of values until satisfactory results were achieved. The threebranch equivalent circuit (per phasel for a three-winding transformer is shown in Fig. 6.4, with the final values shown in Table 6.1 for each transformer. The values are referred to the rectifier side of the transformers. The negative quantities indicated in the table depend on the actual physical arrangement of the windings on the core (ref. 23). It was found to be not feasible to balance the winding resistance further, the inductance being deemed the more important. 


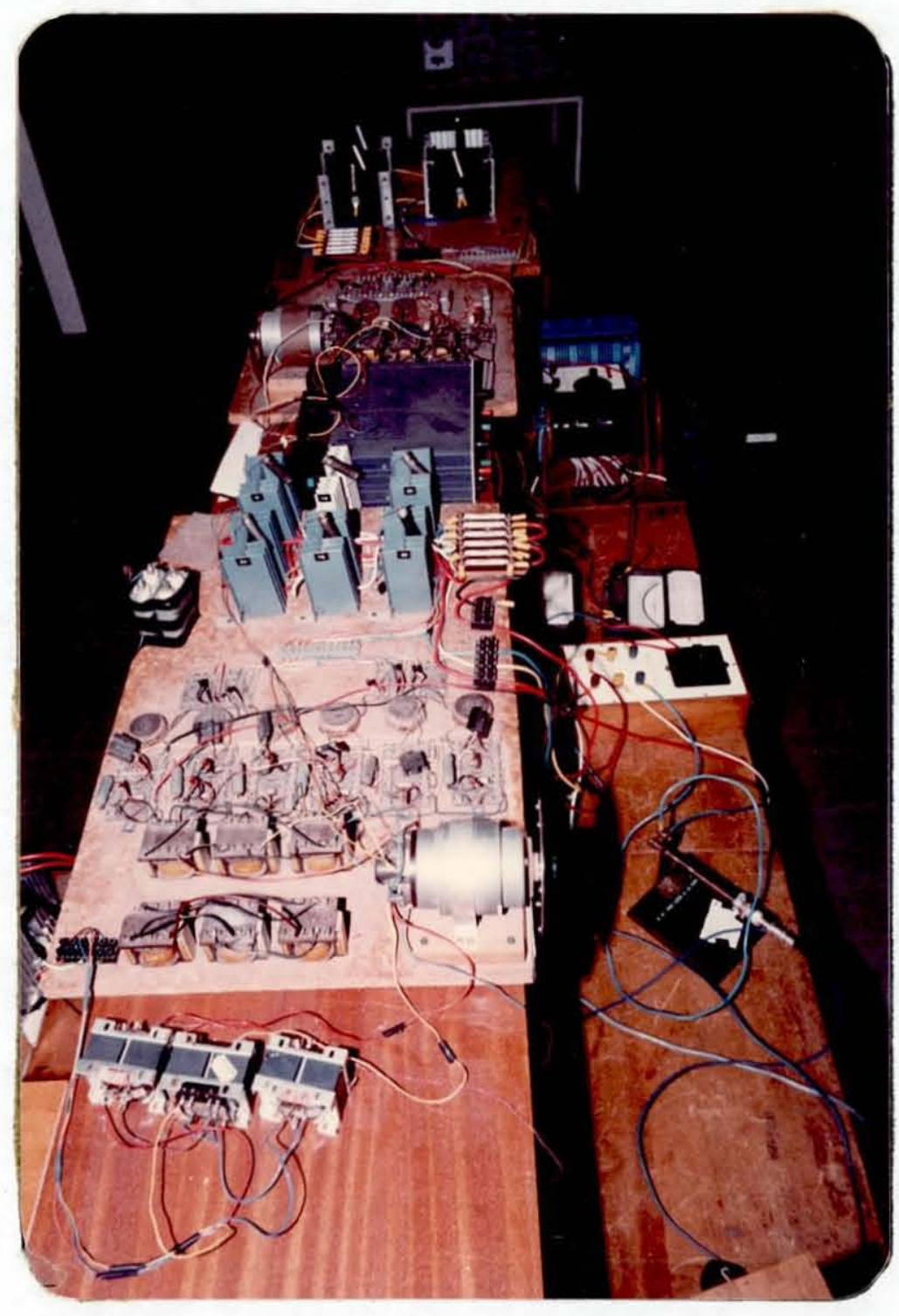

FIG. 6.1 THE EXPERIMEITAL SYSTEM OF THE 12-PULSE PARALLEL CONVERTOR 


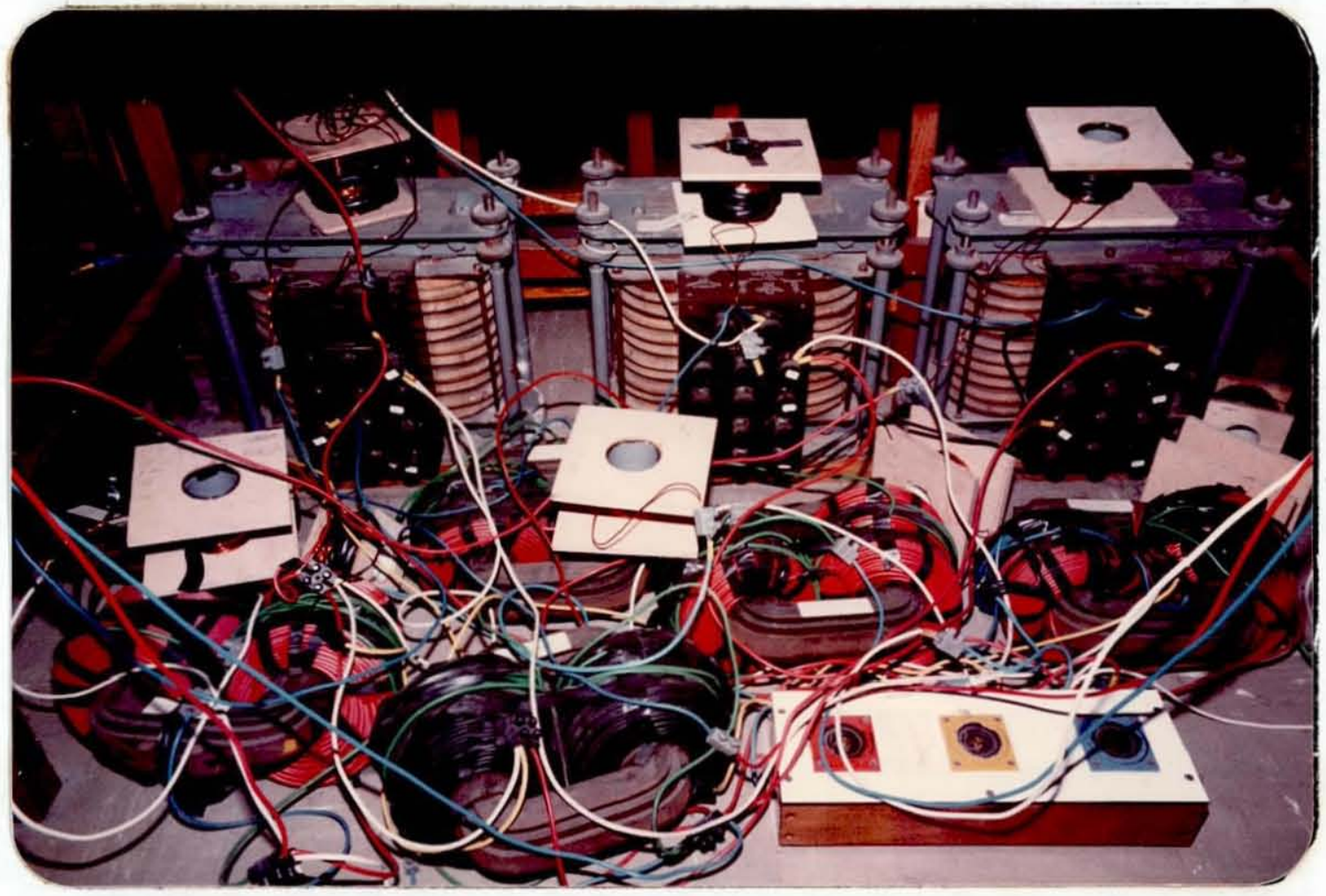

FIG. 6.2 THE THREE SINGLE PHASE THREE-WINDING SUPPIY TRANSFORMER 


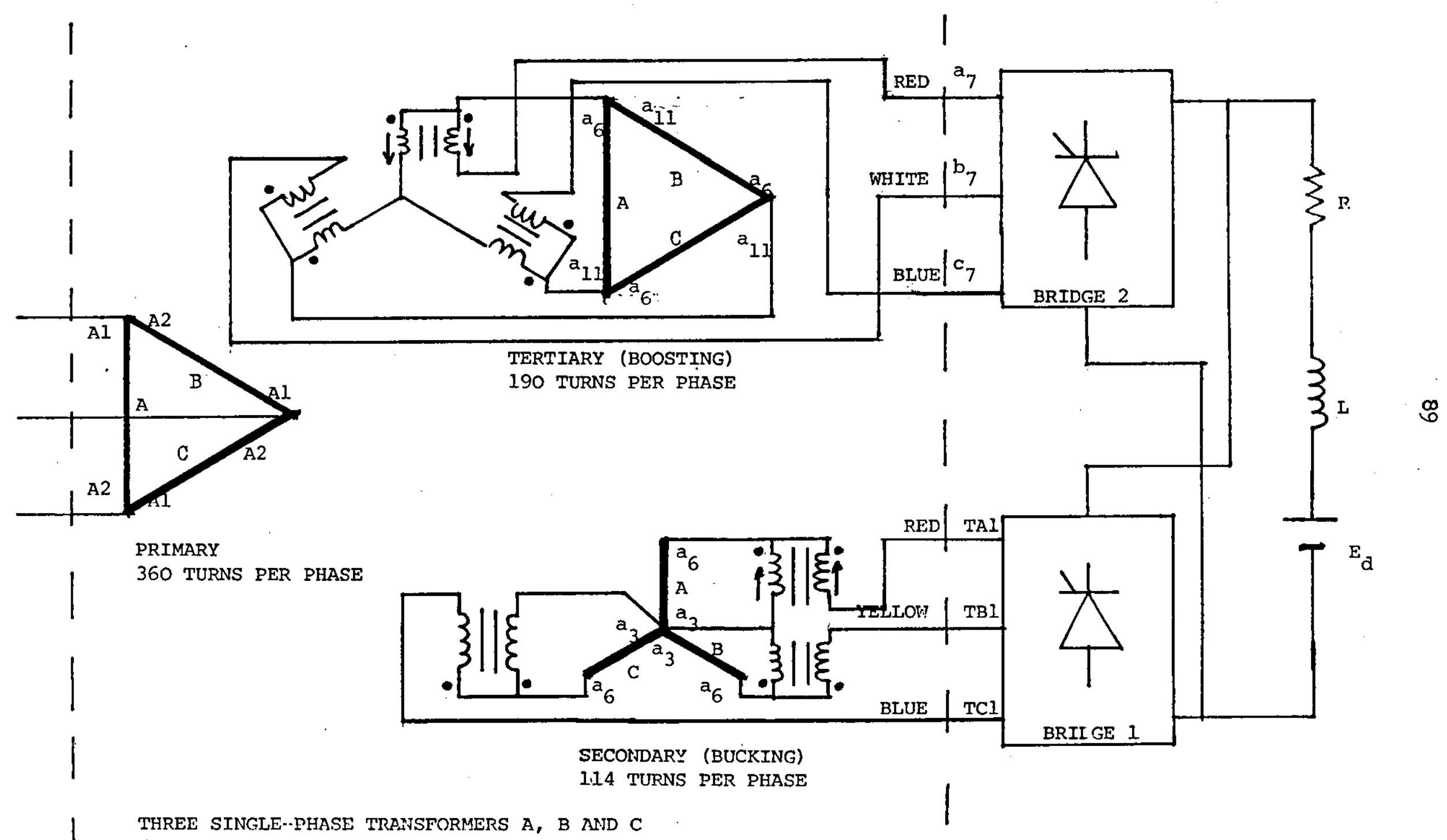

${ }^{\left(a_{6}\right.}$ terminals on the secondary and tertiary windings are separate and isolated)

FIG. 6.3 Three-Phase Connection of Single-Phase Transformers, with Additional Bucking/Boosting Transformers (Actual Connections). Bridge 1 Voltage leads Bridge 2 voltage by $30^{\circ}$ 


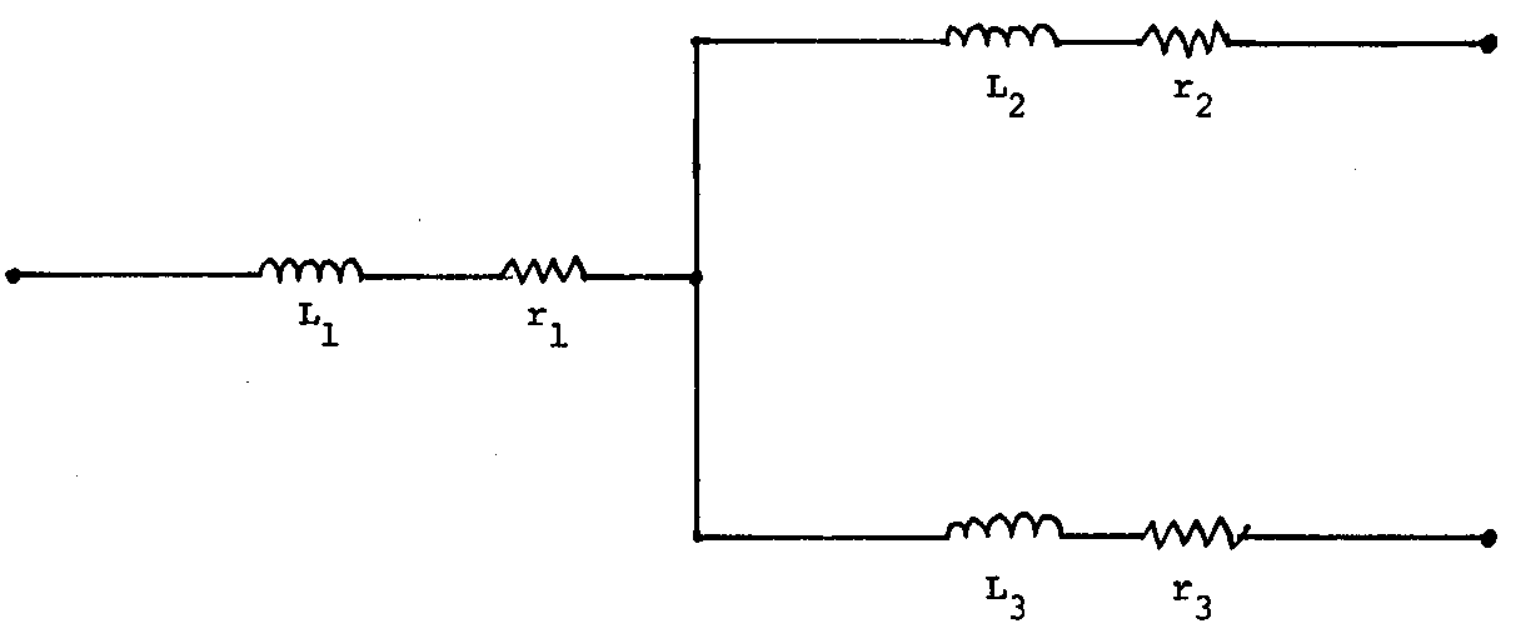

FIG. 6.4 Conventional Equivalent Circuit of a 3-winding Transformer (Drawn per phase) 


\begin{tabular}{|c|c|c|c|}
\hline $\begin{array}{l}\text { Branch } \\
\text { Parameters }\end{array}$ & Transformer A & Transformer B & Transformer C \\
\hline$r_{1}(\Omega)$ & -0.266 & -0.207 & -0.237 \\
\hline$I_{1}(\mathrm{mH})$ & -3.075 & -3.024 & -3.177 \\
\hline$r_{2}(\Omega)$ & 1.096 & 0.962 & 0.895 \\
\hline$L_{2}(\mathrm{mH})$ & 8.321 & 8.27 & 8.203 \\
\hline$r_{3}(\Omega)$ & 0.994 & 0.895 & 0.949 \\
\hline$I_{3}(\mathrm{mH})$ & 8.407 & 8.27 & 8.27 \\
\hline
\end{tabular}

Table 6.1 Measured Transformer Equivalent Parameters (Ref. Fig. 6.4)

\begin{tabular}{|c|c|c|c|c|c|}
\hline \multicolumn{3}{|c|}{ Star secondary (V) } & \multicolumn{3}{|c|}{ Delta secondary (V) } \\
\hline$R-Y$ & $Y-B$ & $B-R$ & $R-Y$ & $Y-B$ & $B-R$ \\
\hline 129.5 & 129.8 & 129.0 & 129.0 & 129.8 & 129.5 \\
\hline
\end{tabular}

Table 6.2 Open-circuit Transformer Secondary Iine Voltages. 
The open-circuit secondary voltages are given in Table 6.2 for an applied primary voltage of $240 \mathrm{~V}$, balanced to within $1 \mathrm{~V}$. An average value of $129.4 \mathrm{~V}$ has been used in the program. The effective transformation ratios for each secondary phase to primary phase as defined in Figs. 3.11 and 3.12 are:

$$
\begin{aligned}
& \frac{\mathrm{N}_{4}}{\mathrm{~N}_{1}}=0.5392 \\
& \frac{\mathrm{N}_{7}}{\mathrm{~N}_{1}}=0.3113
\end{aligned}
$$

As noted in Chapter 3 , the equivalent circuit parameters of the form given in Table 6.1 were not used in the analysis, but were replaced by the self and mutual inductances of the windings. The calculation of these values from the circuit measurements is given in Appendix I. Again, inevitably, there is small unbalance between the windings, and average values were adopted for the program. These are presented in Table 6.3.

The control circuitry for the parallel bridge convertor and the thyristor gate drive circuits are given in Appendix II. Adjustment of thyristor firing delay is made by a phase-shifting transformer (magslipl. with a protractor to indicate directly the firing delay angle, Each bridge is fitted with its own magslip, but the connection is such that one operates as a master firing angle controller for both bridges (this being Bridge 1 magslip), while the other is set to give a $30^{\circ}$ firing delay to the Bridge 2 thyristors relative to those of Bridge 1. This latter magslip may be used to trim the firing of the Bridge 2 thyristors for best balance between the bridges. The measuring equipment includes dc meters of the moving coil type and ac-dc meters of the moving iron type, connected respectively 


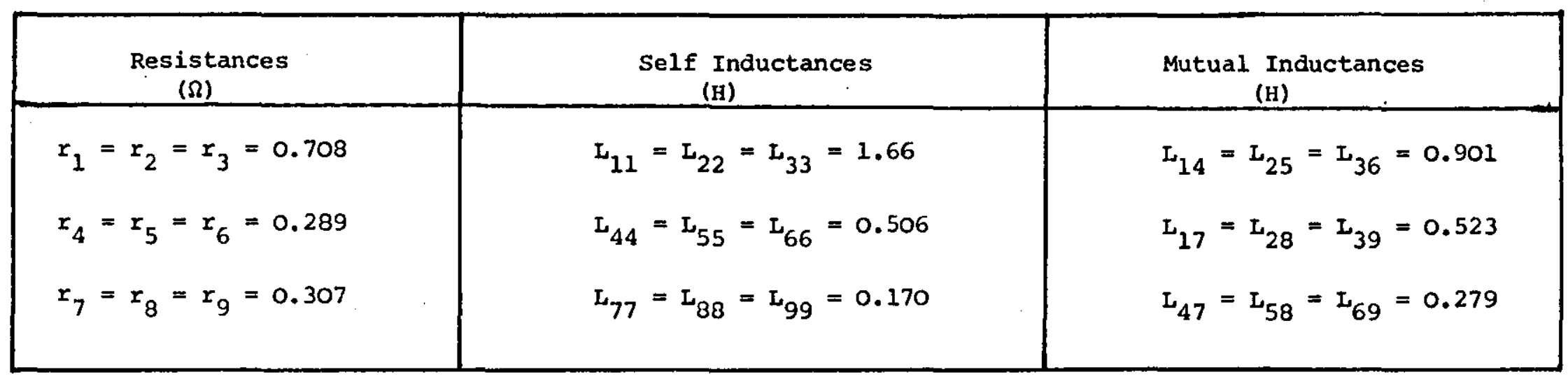

Table 6.3 Transformer Data used in the Mathematical Model

(Ref. Fig. 3.7a, b and c) 
on the dc and ac sides. To aid the measurement of the current using the oscilloscope or its harmonic contents, a coaxial shunt of $0.01 \Omega$ resistance has been used. Care has to be taken when measuring the primary side current as the oscilloscope chassis is usually earthed. A Tektronix type 555 dual beam oscilloscope has been used. The harmonic contents of the current are measured using the Brüel and Kjaer frequency analyser type 2105.

The device modelling data used in the mathematical model is as follows :

$$
\begin{aligned}
& \mathrm{e}_{\text {thy }}=0 \\
& \mathrm{~L}_{\text {thy }}=0 \\
& \mathrm{R}_{\text {thy }}=0.1 \Omega
\end{aligned}
$$

\subsection{Alternating current waveforms}

For the purpose of waveform prediction, four firing delay angles have been chosen to cover the four operating modes of which the experimental system is capable. With delay angles of $160^{\circ}, 150^{\circ}$, $120^{\circ}$ and $60^{\circ}$, which correspond to Modes $1,2,3$ and 4 respectively, both with and without load back emf present, (except in Mode 1, the delay angle for the latter is $165^{\circ}$, the following current waveforms are predicted:

(a) The two bridge supply line currents;

(b) the tertiary winding current;

(c) the supply line current;

(d) the primary phase current;

Additionally the mean dc output voltage and current values are computed. 
The ac supply voltage to the rectifier system is measured to be 339.4 peak or $240 \mathrm{~V}$ rms with a frequency of $50 \mathrm{~Hz}$. The computer program runs with a step length of 0.0001 seconds, this being chosen by trial and error to minimise the computing time and provide acceptable accuracy in the final results, as mentioned in Chapter 4.

The load side is represented by a variable resistor, an inductor and a battery connected in series. The load inductance was measured at $100 \mathrm{~Hz}$ using the digital Marconi bridge meter. For the model, the load resistance used in the computation is the dc resistance which is simply calculated from the knowledge of the dc mean output voltage, $v_{d^{\prime}}$ the dc mean output current $I_{d}$ and the battery open circuit voltage $E_{d}$ and from,

$$
R=\frac{v_{d}-E_{d}}{I_{d}}
$$

The numerical values are shown below. The following gives the load data used in the experiment and prediction of the currents:

$$
\mathrm{R}=5.1 \Omega \quad \mathrm{L}=0.0174 \mathrm{G} \quad \mathrm{E}_{\mathrm{d}}=24.0 \mathrm{~V} \text { or oV }
$$

The predicted current waveforms and the oscillograms obtained from the laboratory system are given in Fig. 6.5 to Fig. 6.20. The shapes of the bridge supply current waveforms correspond with those put forward in Chapter 2 and the individual transformer winding currents have the form expected by derivation from the bridge currents. Correlation between computed and observed current waveforms is very good. Amplitudes correspond for each operating mode within experimental error, although there are small differences in the conduction intervals. Mean dc values of the output currents, $I_{d 1}$ and $I_{d 2}$ respectively for Bridge 1 and Bridge 2 , and the output voltage $v_{d^{\prime}}$ are given with the bridge supply current waveforms. The measured and the predicted 
Frequency $=58.8 \mathrm{hz}$ and alpha $=168.0$ deg. RL $=5.10800$ ohas $L=0.017480800 \mathrm{~h}$

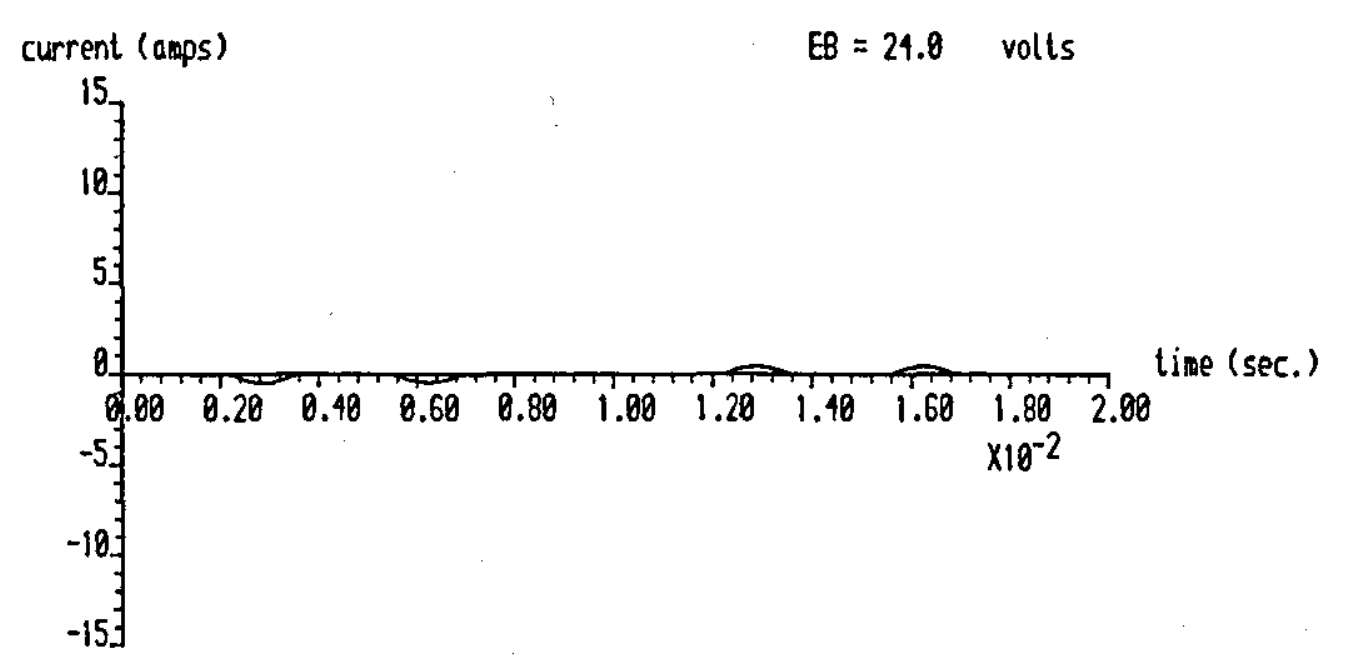

FIG. 6.5a THE ERIDGE SUPPLY LINE CURRENT

$$
\begin{aligned}
& I_{d 1}=0.1 \mathrm{~A} \quad I_{d 2}=0.1 \mathrm{~A} \quad I_{d}=0.2 \mathrm{~A} \\
& V_{d}=26 \mathrm{~V}
\end{aligned}
$$

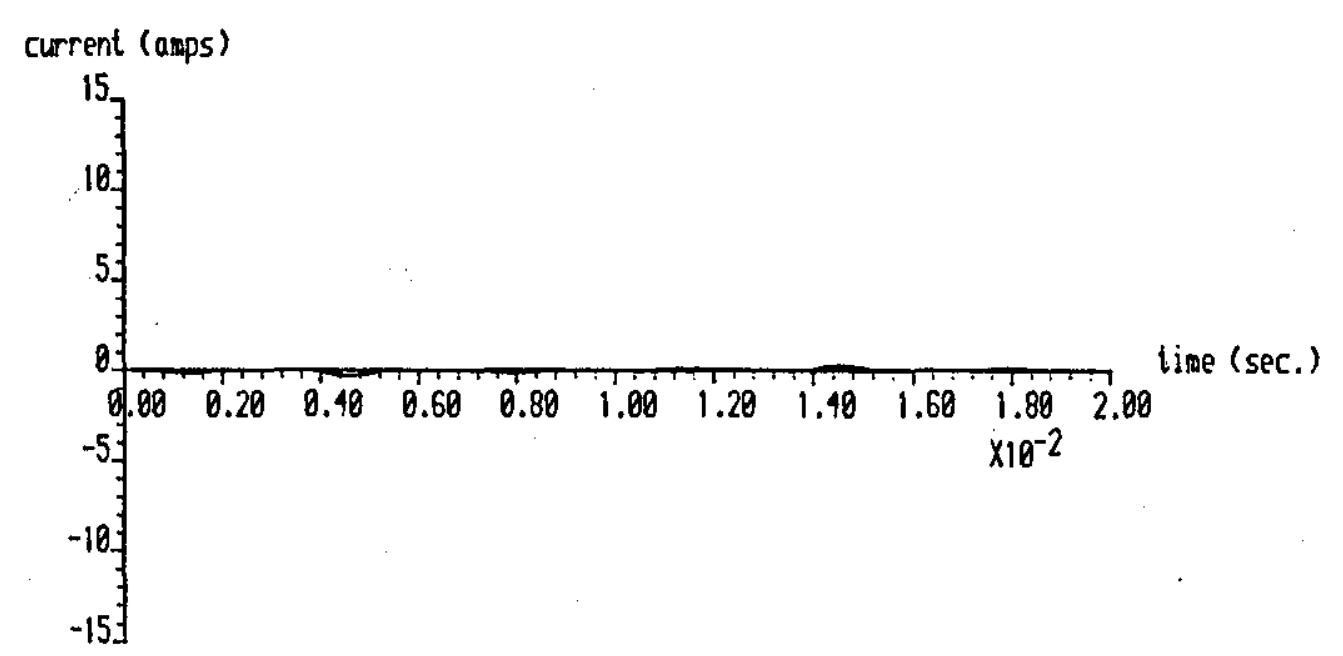

FIG. $6.5 \mathrm{~b}$ THF TERTARY WINDING CURRENT

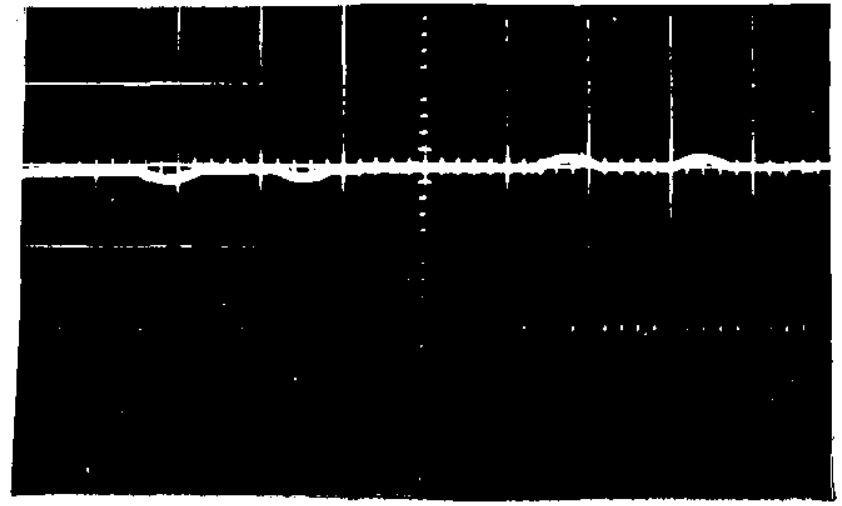

FIG. 6.6a THE BRIDGE SUPYIY CURRENT

$I_{d 1}=0.15 \mathrm{~A} \mathrm{I} I_{d 2}=0.2 \mathrm{~A} \quad I_{d}=0.4 \mathrm{~A}$ $v_{d}=28.5 \mathrm{~V}$

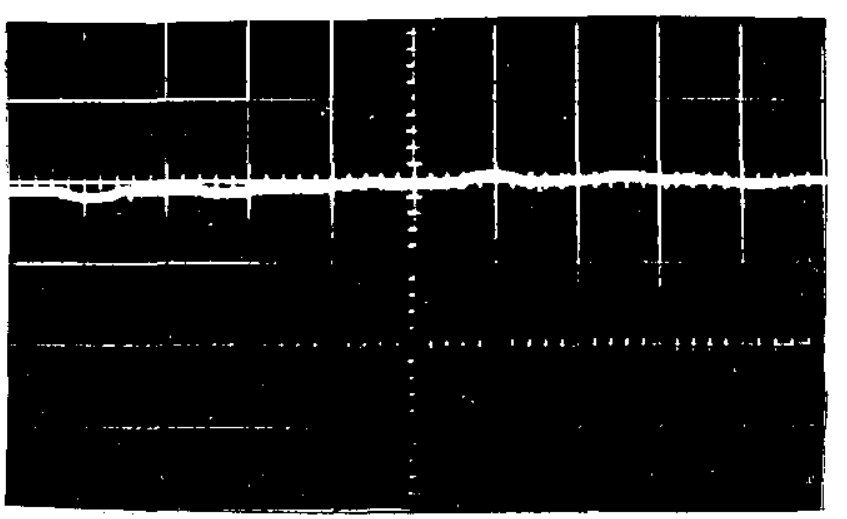

FIG. 6.6b THE TERTIARY WINDING CURRENT

THE OSCILLCGRAMS

Scale: Frorizontal - 2 ms per division Vertical - 5 A per division 
Frequency $=50.0 \mathrm{hz}$ and aipho $=160.0$ deg. $\mathrm{RL}=5.10000$ ohms $\mathrm{L}=0.017409000 \mathrm{~h}$

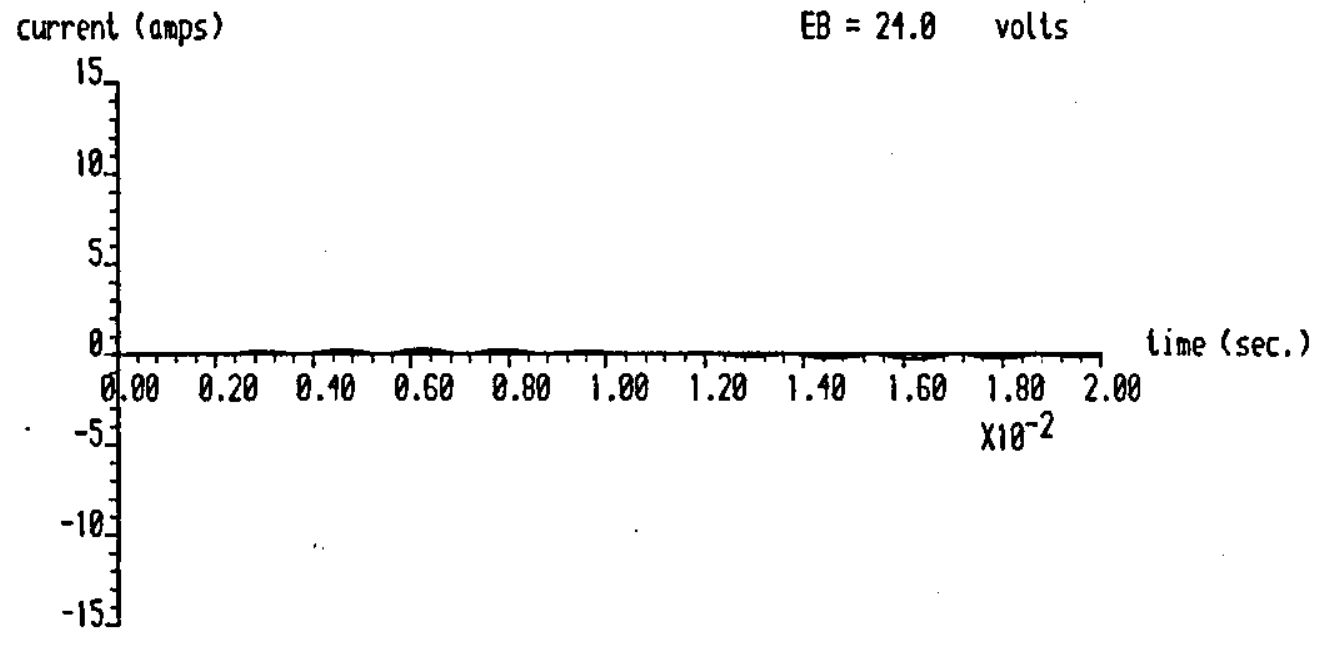

FIG. $6.5 \mathrm{c}$ THE STIPPLY LINE CURRENT

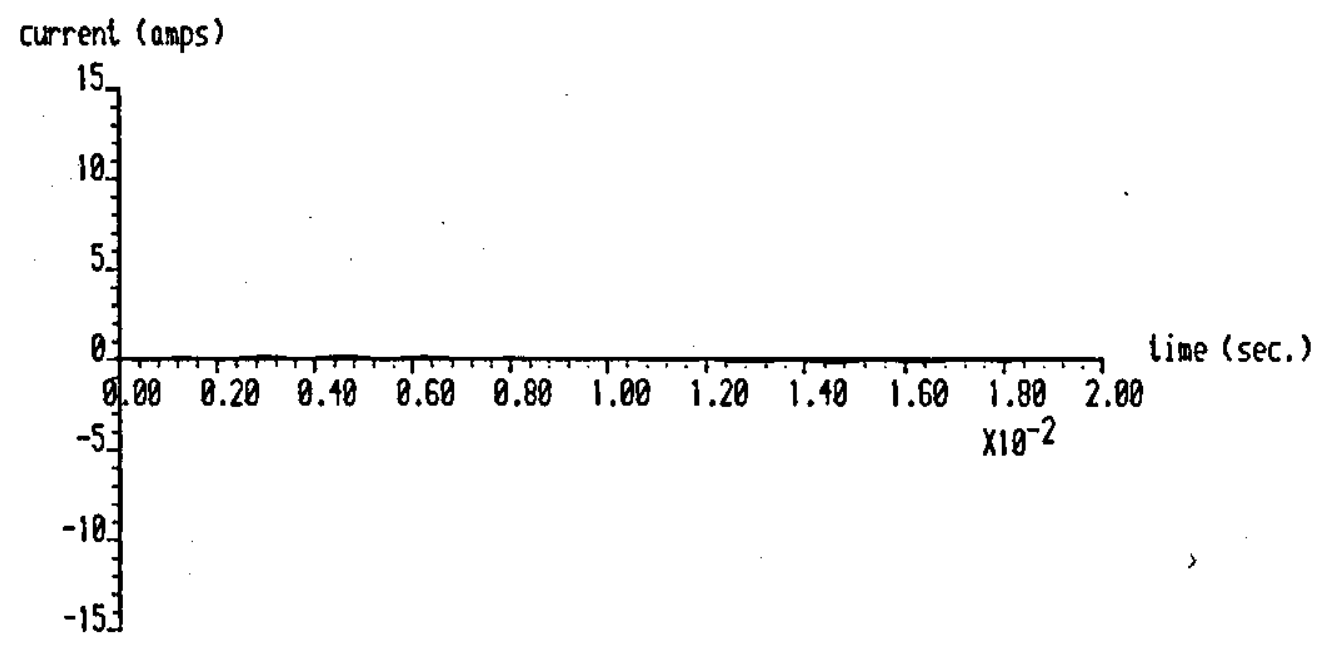

FIG. 6.5d THE PRIMARY PHASE CURRENT

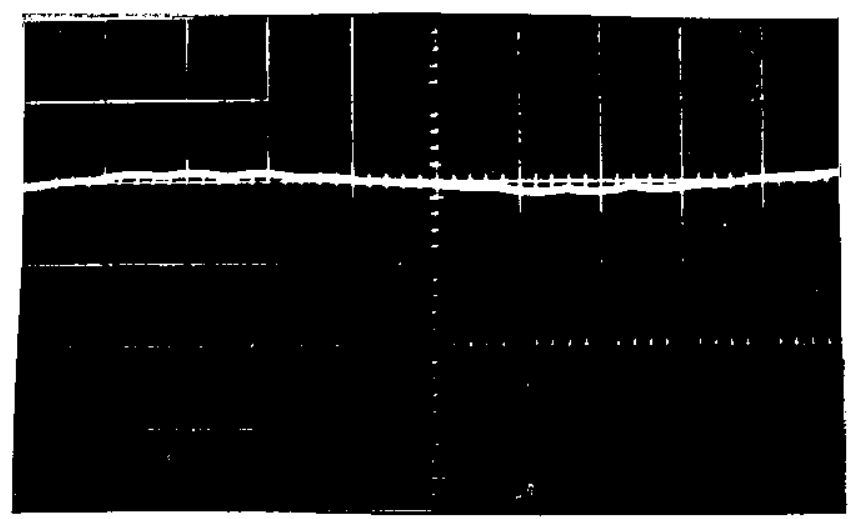

FIG. $6.6 \mathrm{C}$ THE SUPPIY LINE CUPRENT

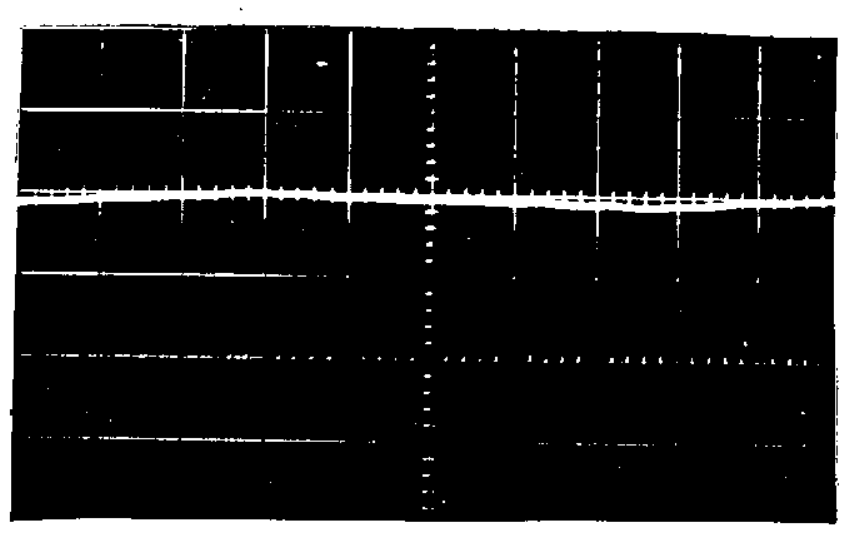

FIG. 6.6d THF PRIMARY PHASE CIYPENN

THE OSCILIOGRAMS

Scale: Horizontal - $2 \mathrm{~ms}$ per division Vertical - 5 A per division 
Frequency $=50.8 \mathrm{hz}$ and $\mathrm{alpha}=150.0 \mathrm{deg}$. RL $=5.10000$ ohms $L=0.017408000 \mathrm{~h}$

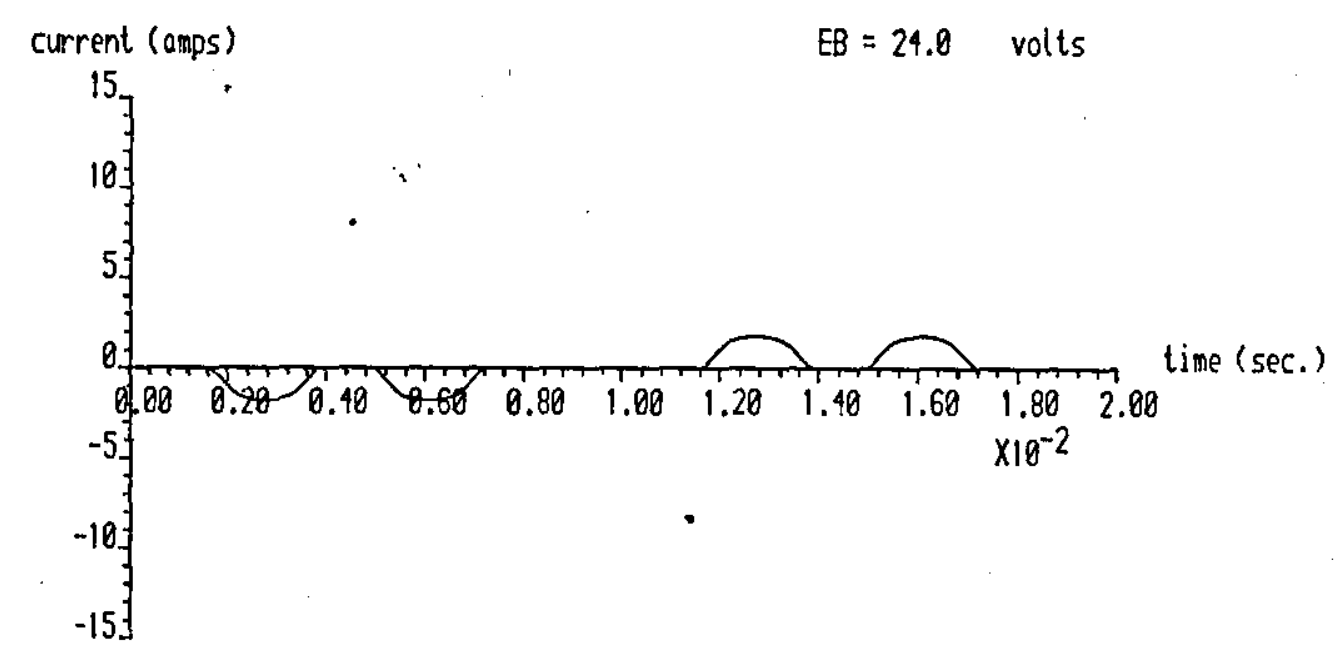

FIG. 6.7a THE BRIDGE SUPPLY LINE CURRENT

$\begin{aligned} \therefore I_{d_{1}} & =0.75 \mathrm{~A} \quad I_{d 2}=0.75 \mathrm{~A} \mathrm{I} I_{d}=1.5 \mathrm{~A} \\ V_{d} & =32.4 \mathrm{~V}\end{aligned}$
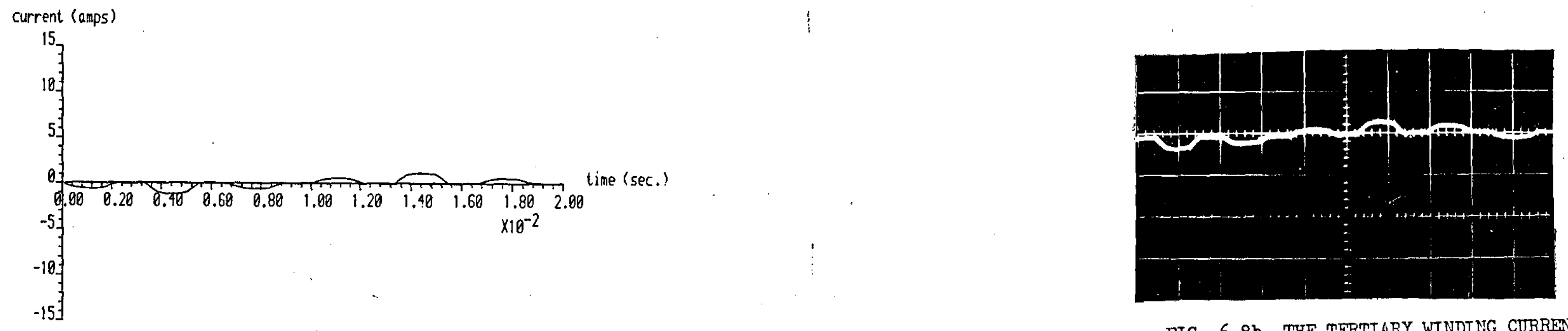

FIG. 6.7b THE TERTIARY WINDING CURRENT

FIG. 6.8b THE THRTIARY WTNDING CURRENT THF OSCILLOGRAMS

Scale: Horizontal - $2 \mathrm{~ms}$ per division Vertical - 5 A per division

$I_{d 1}=0.9 \mathrm{~A} \quad I_{d 2}=1.05 \mathrm{~A} \quad I_{d}=2.0 \mathrm{~A}$

$\mathrm{V}_{\mathrm{d}}=37.5 \mathrm{~V}$ 
Frequency $=58.0 \mathrm{hz}$ and alpha $=150.0 \mathrm{deg} . \mathrm{RL}=5.10800$ ohms $\mathrm{L}=0.017400000 \mathrm{~h}$

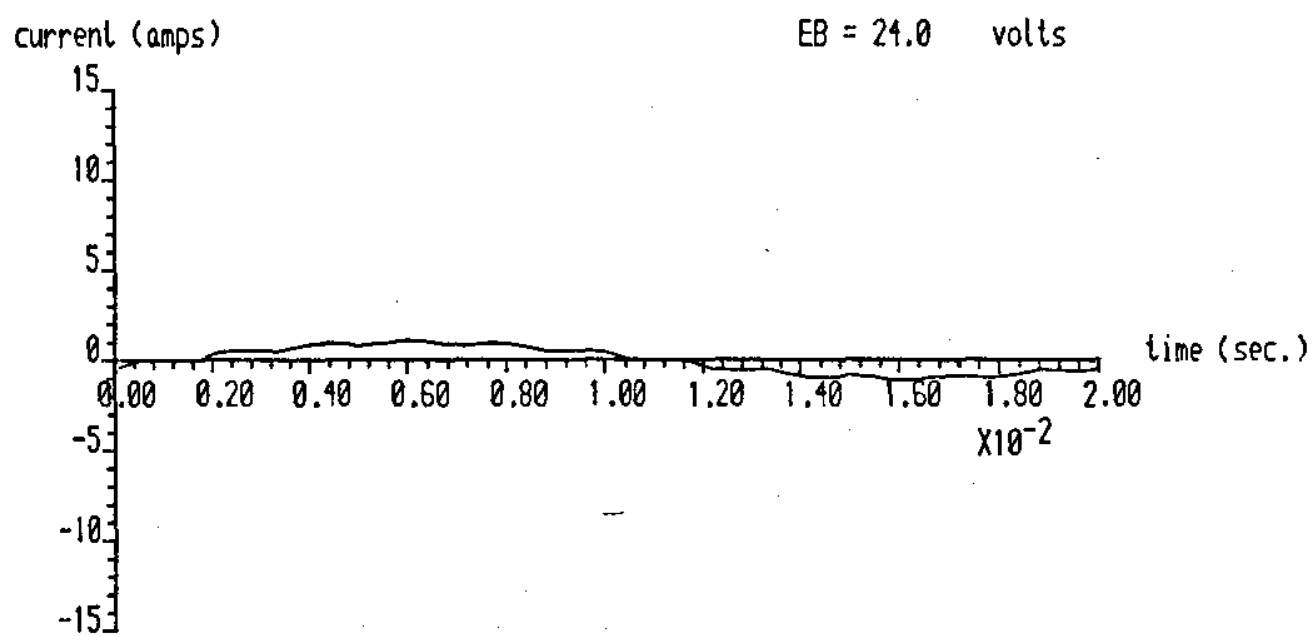

FIG. $6.7 \mathrm{c}$ THE STPPLY IINE CURRENT

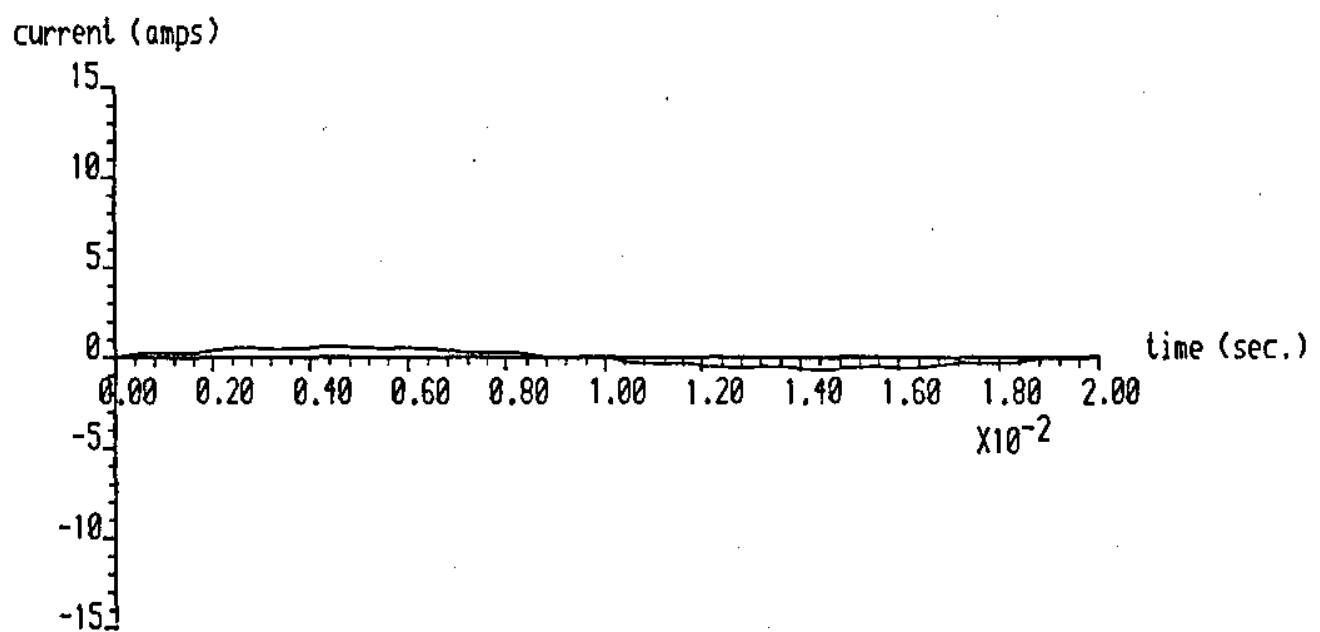

FIG. 6.7d THE PRIMARY PHASE CURRENT

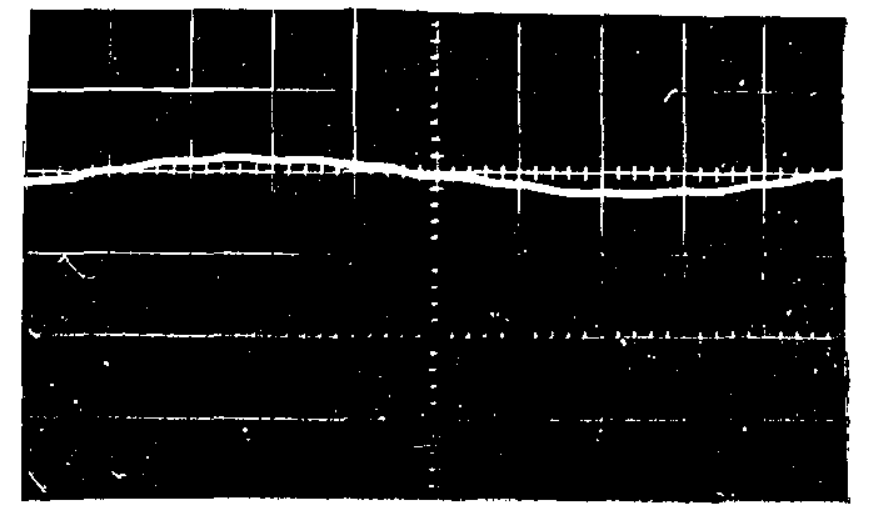

FIG. 6.8C THE SUPPLY IJPE CURRENT

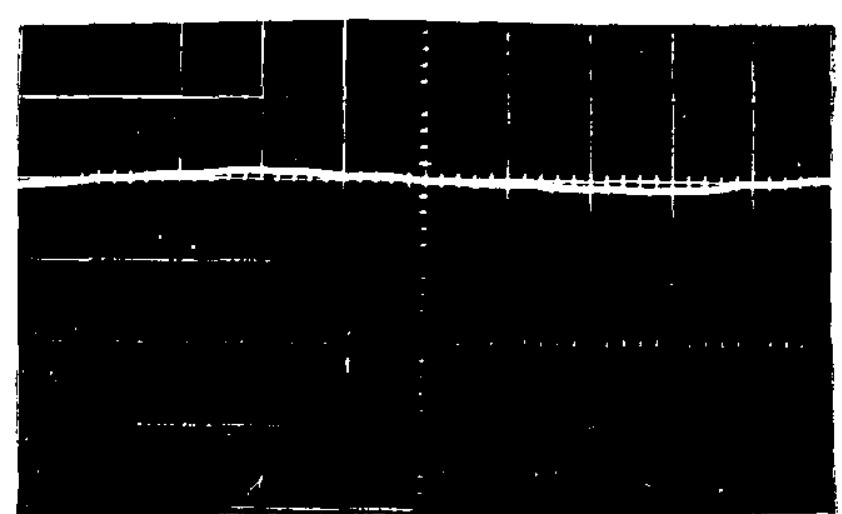

FIG. 6.8d THE PRIMLARY PHASE CITRETT THE OSCILLOGRAMS

Scale: Horizontal - $2 \mathrm{~ms}$ per division Vertical - 5 A per division 
Frequency $=50.8 \mathrm{hz}$ and alpha $=120.8$ deg. RL $=5.10000$ ohms $L=0.017400000 \mathrm{~h}$

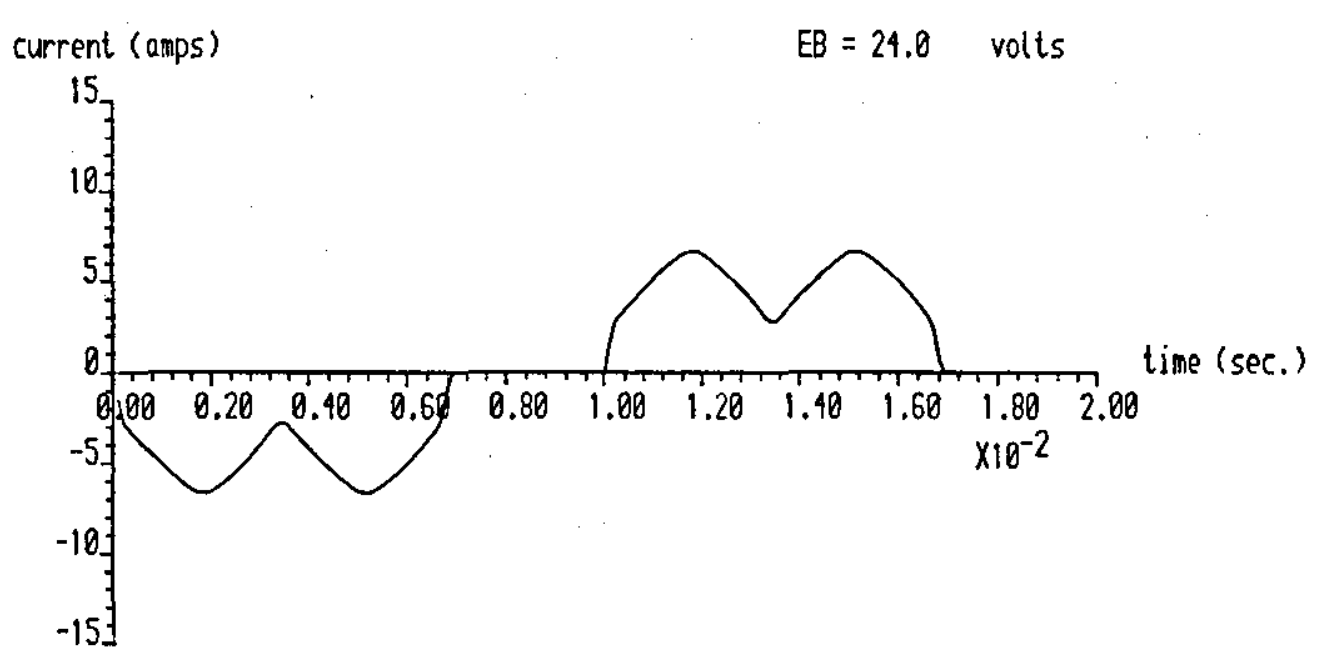

FTG. 6.9a THF, RRJDGE SUPPLY LINE CURRENTT

$I_{\mathrm{d} 1}=4.78 \mathrm{~A} \quad I_{\mathrm{d} 2}=5.46 \mathrm{~A} \quad I_{d}=10.24 \mathrm{~A}$

$v_{d}=76.8 \mathrm{~V}$

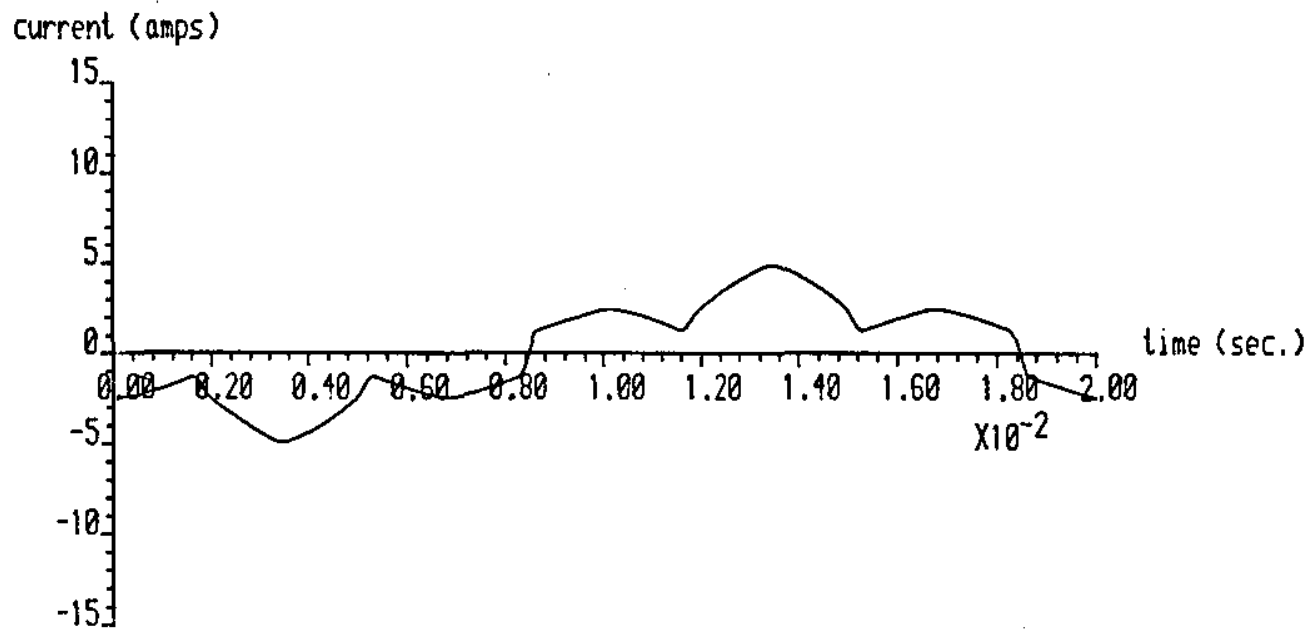

FIG. $6.9 \mathrm{~b}$ THE TEPTIARY WINDING CURRENT

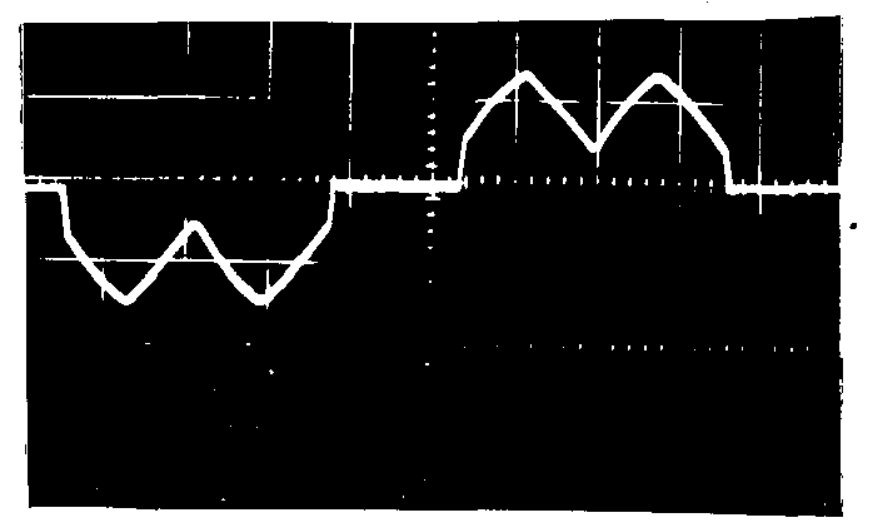

FIG. 6.10a THE RRIDGE SUFPLY IINE CURRENT $I_{\mathrm{d} 1}=5.2 \mathrm{~A} \quad I_{\mathrm{d} 2}=5.25 \mathrm{~A}=10.4 \mathrm{~A}$ $\mathrm{v}_{\mathrm{d}}=81 \mathrm{~V}$

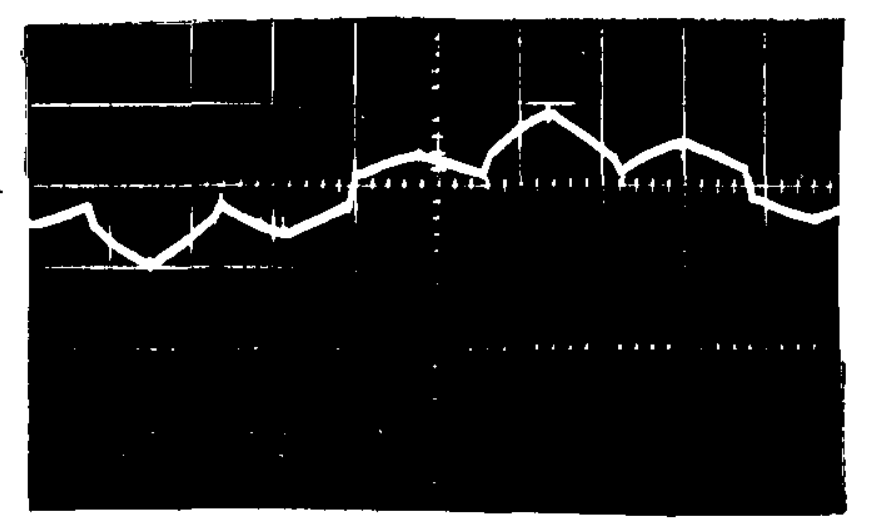

FIG. 6.10b THE TERTIARY WINDING CURRENT THE OSCILIOGKANS Scale: Horizontal - $2 \mathrm{~ms}$ per division Vertical - 5 A per division 
Frequency $=50.0 \mathrm{hz}$ and alpha $=120.0 \mathrm{deg} . \mathrm{RL}=5.10000$ ohms $\mathrm{L}=0.017400000 \mathrm{~h}$

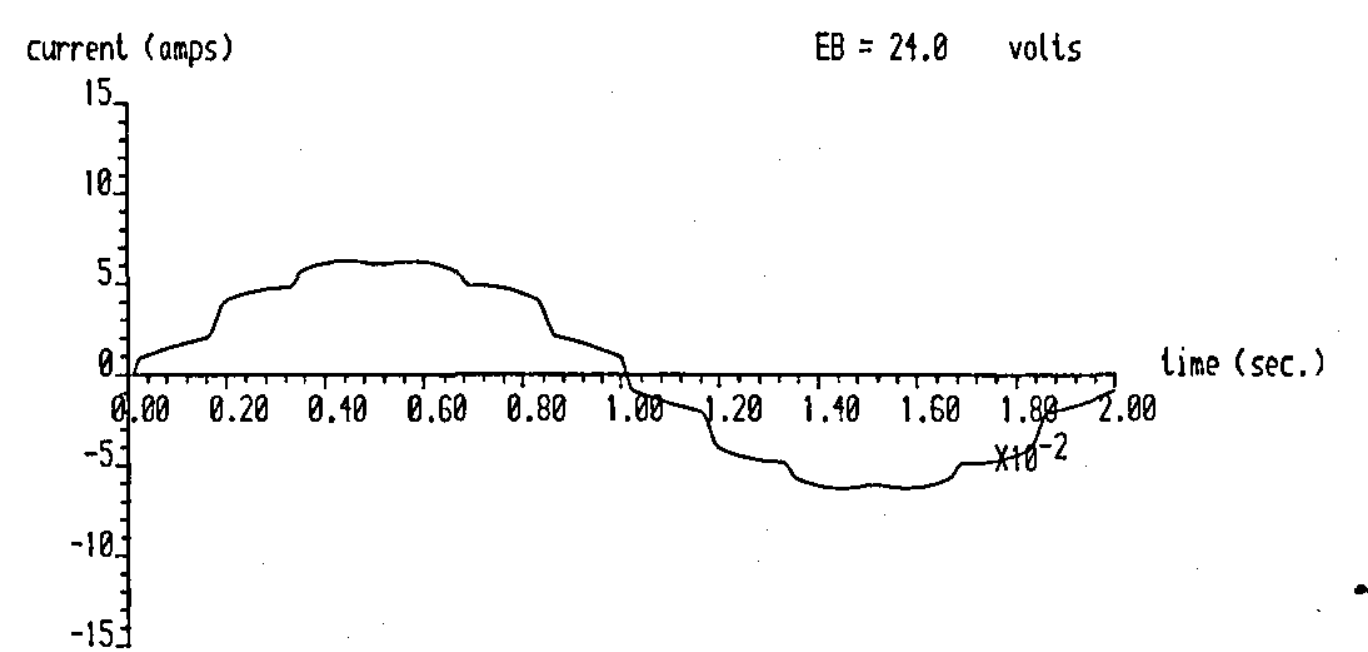

FIG. 6.9c THE SUPPLY LINE CURRENT

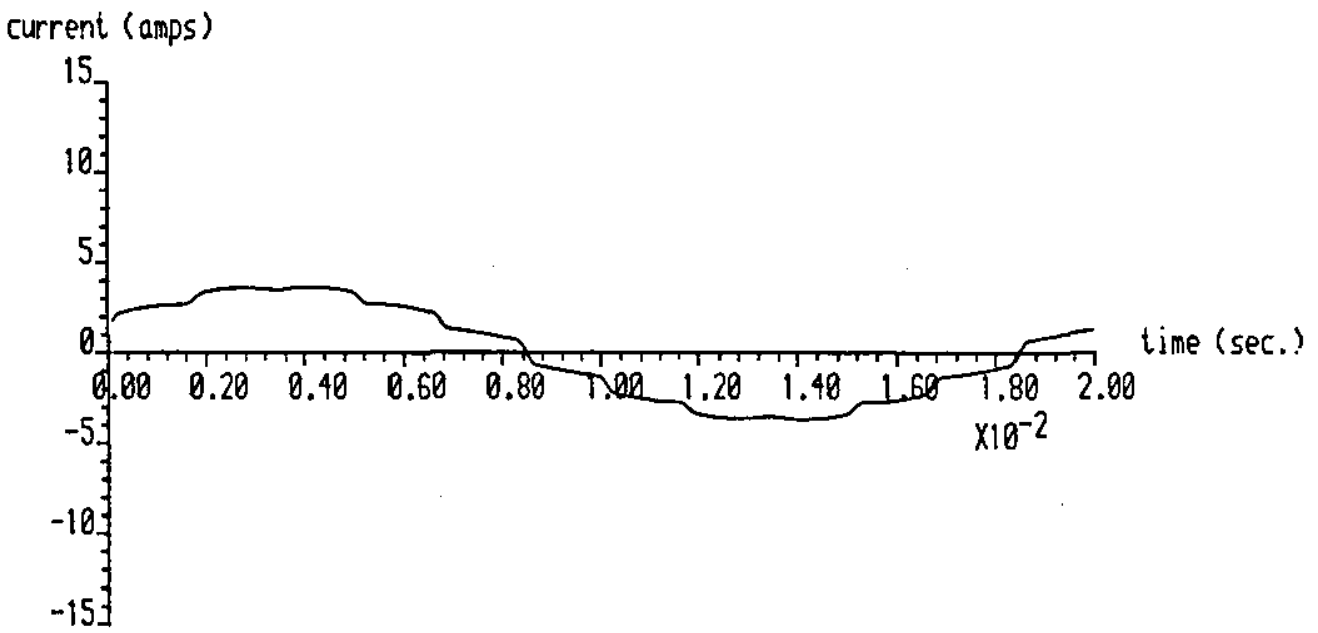

FIG. 6.9d THE PRIMARY PHASE CURRENT

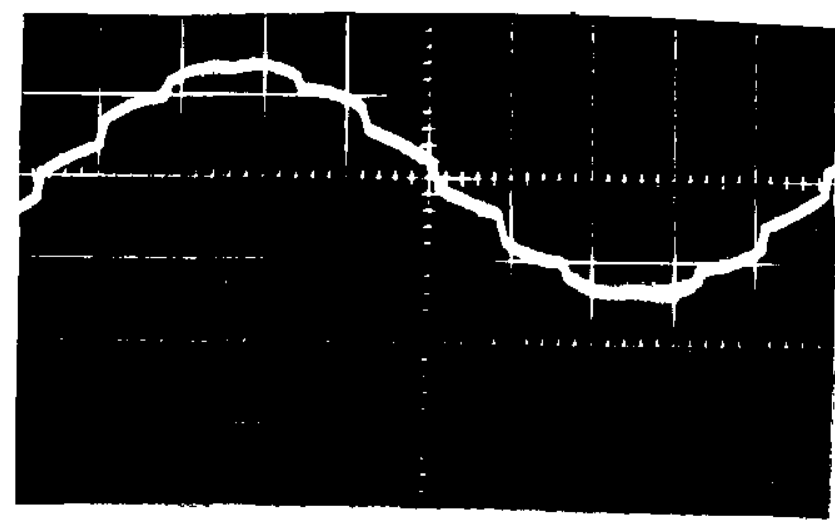

FIG. $6.10 \mathrm{C}$ THE STFPLY LTHE CURRFIT

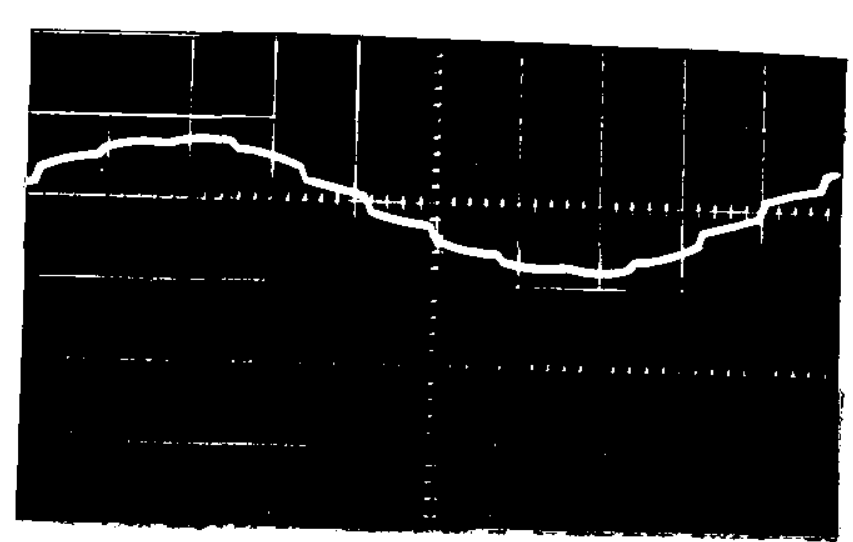

FIG. 6.10d THE PRINARY PHASE CURRENT

THE OSCILLOGRAMS

Scale: Horizontal - $2 \mathrm{~ms}$ per division Vertical - 5 A per division 
Frequency $=50.0 \mathrm{hz}$ and alpha $=60.0$ deg. RL $=5.10000$ ohms $\mathrm{L}=0.017400000 \mathrm{~h}$

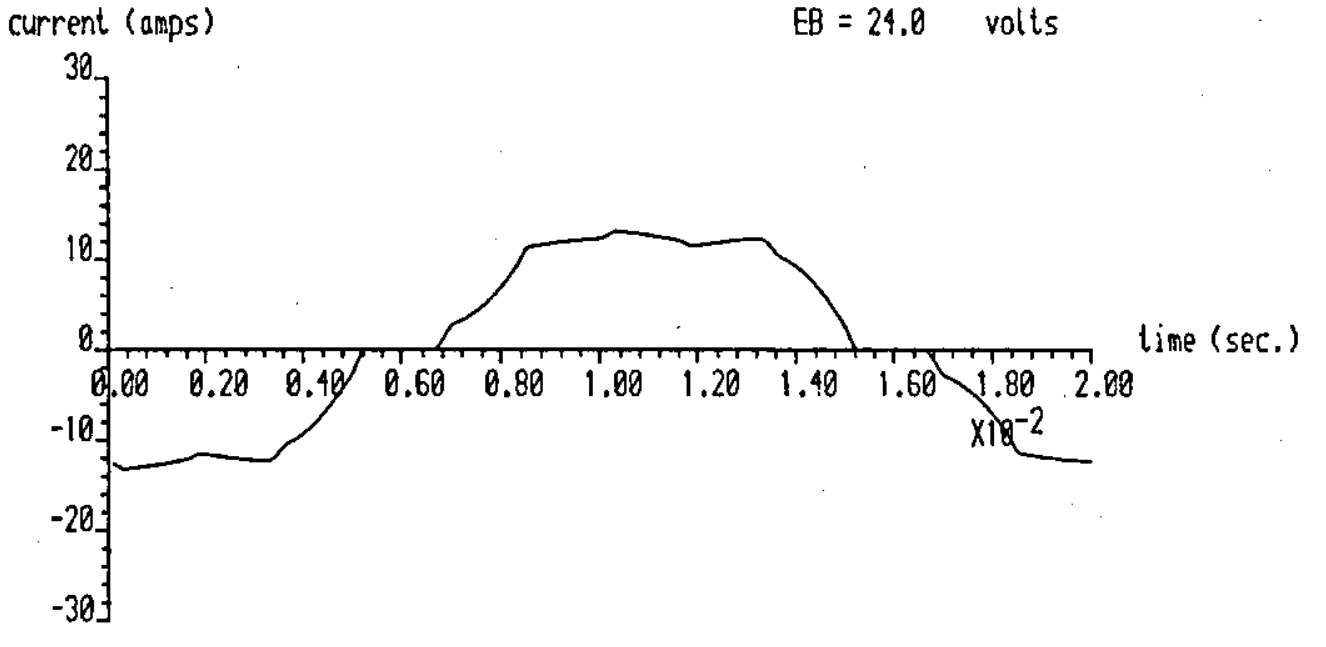

FIG. 6.11a THE BRIDGE SUPPLY LINE CURRENT

$I_{\mathrm{d} 1}=12.17 \mathrm{~A} \quad I_{\mathrm{d} 2}=12.25 \mathrm{~A} \quad I_{\mathrm{d}}=24.42 \mathrm{~A}$

$\mathrm{v}_{\mathrm{d}}=150.1 \mathrm{~V}$

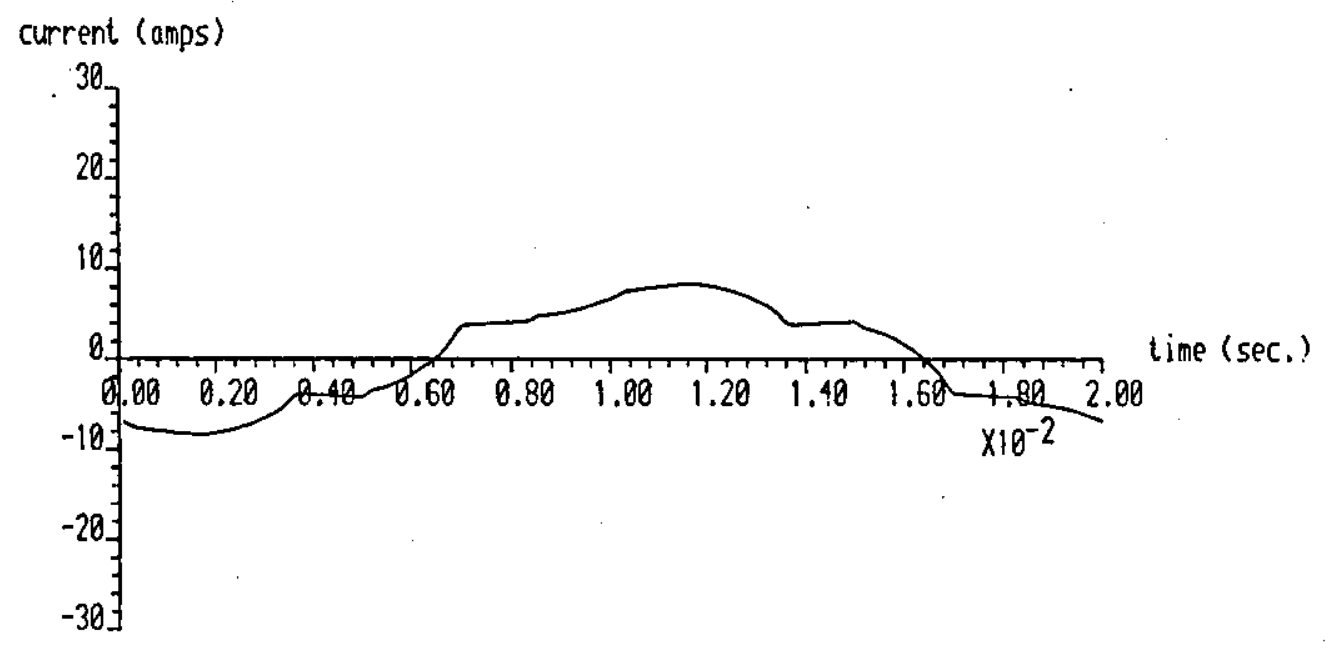

FIG. 6.11b THE TEFTIARY WINDING CURREPTT

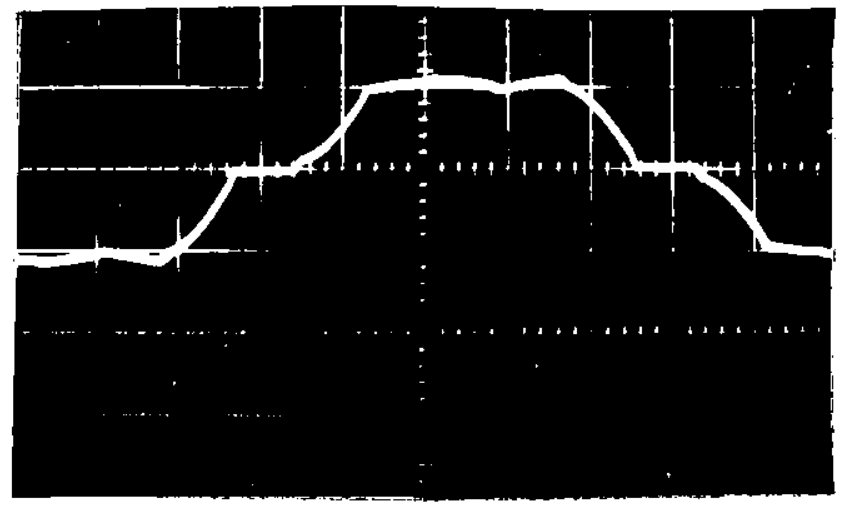

FIG. 6.12a THE RRIDGF SUPPLY LINE CURRENT $I_{d 1}=11.8 \mathrm{~A} \quad I_{d 2}=11.4 \mathrm{~A} \quad I_{d}=23.4 \mathrm{~A}$ $\mathrm{v}_{\mathrm{d}}=144.5 \mathrm{~V}$

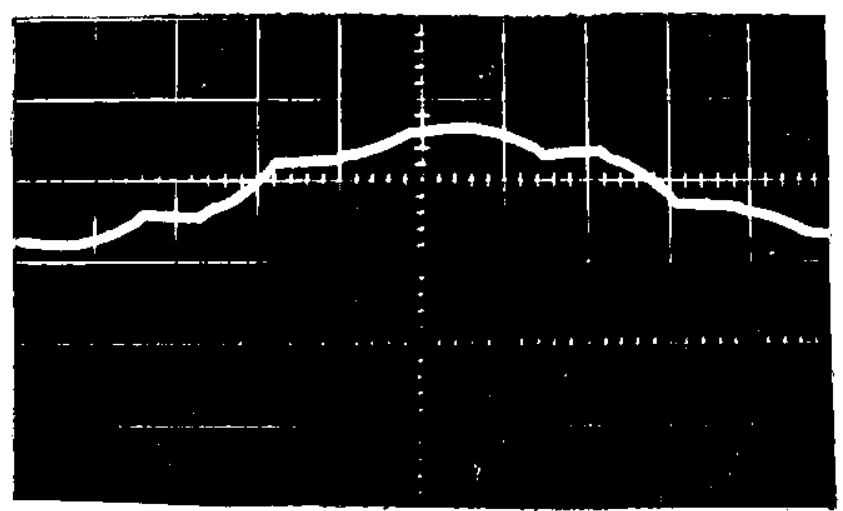

FTG. $6.12 \mathrm{~b}$ TYSE TERTIARY WINDING CURRETT THE OSCILIOGRAMS

Scale: Horizontal - $2 \mathrm{~ms}$ per division Vertical - 10 A per division 
Frequency $=50.0 \mathrm{hz}$ and alpha $=60.0$ deg. $\mathrm{RL}=5.10000$ ohms $\mathrm{L}=0.017400000 \mathrm{~h}$

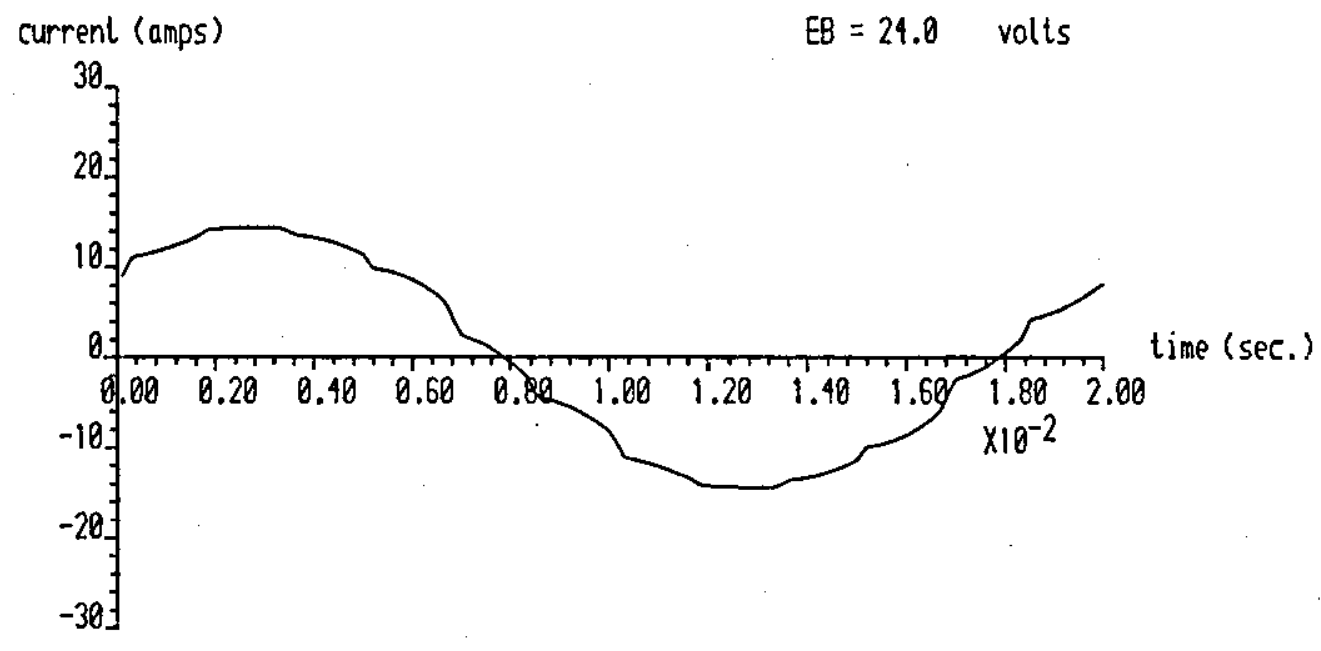

FIG. 6.11C TPE SUPFLY IINE CURRENT

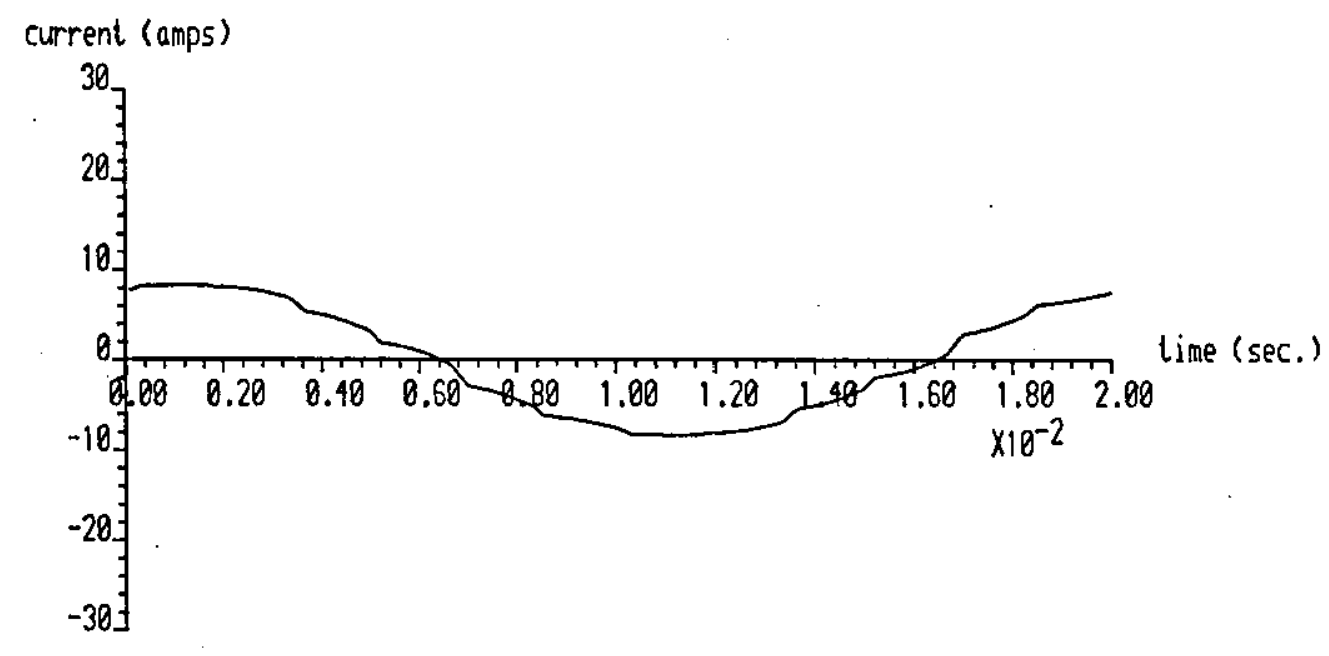

FIG. 6.11d THE PRIMARY PHASE CURRENT

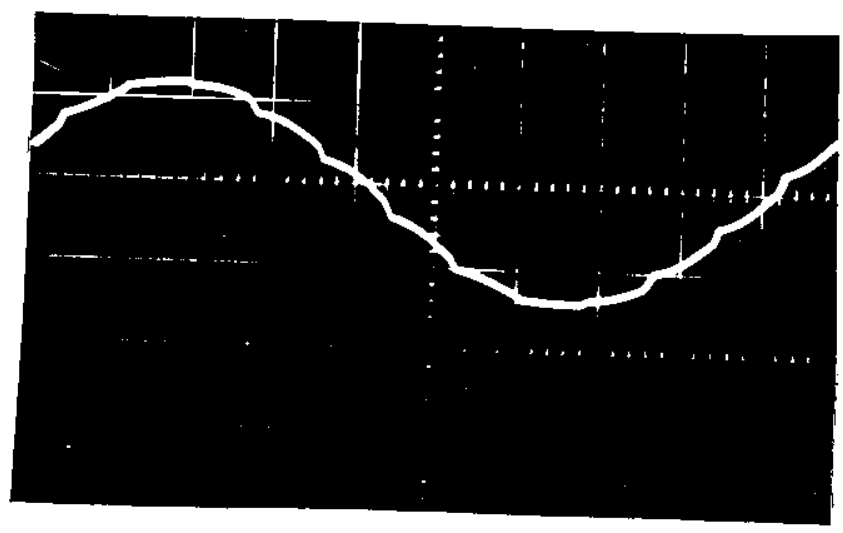

FIG. $6.12 \mathrm{C}$ THE STPPLY IINE CUYRRFNT

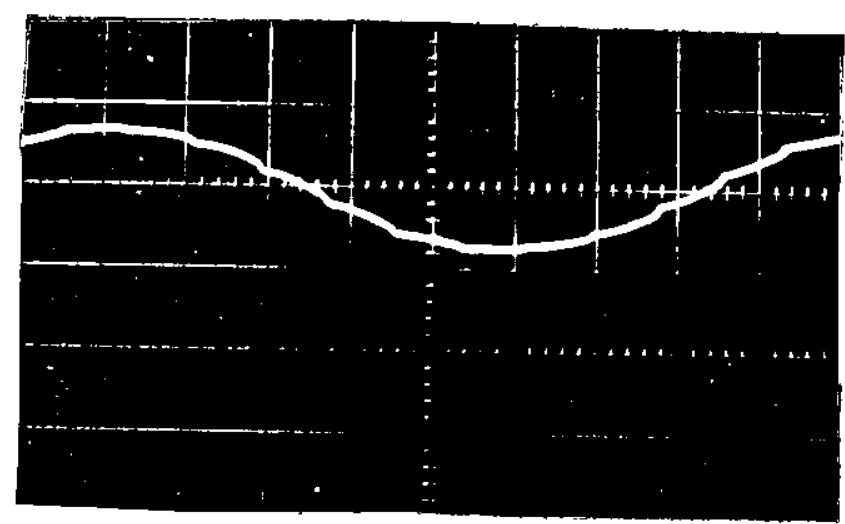

FIG: 6.12d THE FRIMARY PHASF CIRREIT THE OSCILLOGRAMS Vertical - 10 A per division 
Frequency $=50.0 \mathrm{hz}$ and alpha $=165.0 \mathrm{deg}$. RL. $=5.18000$ ohms $L=0.017400000 \mathrm{~h}$

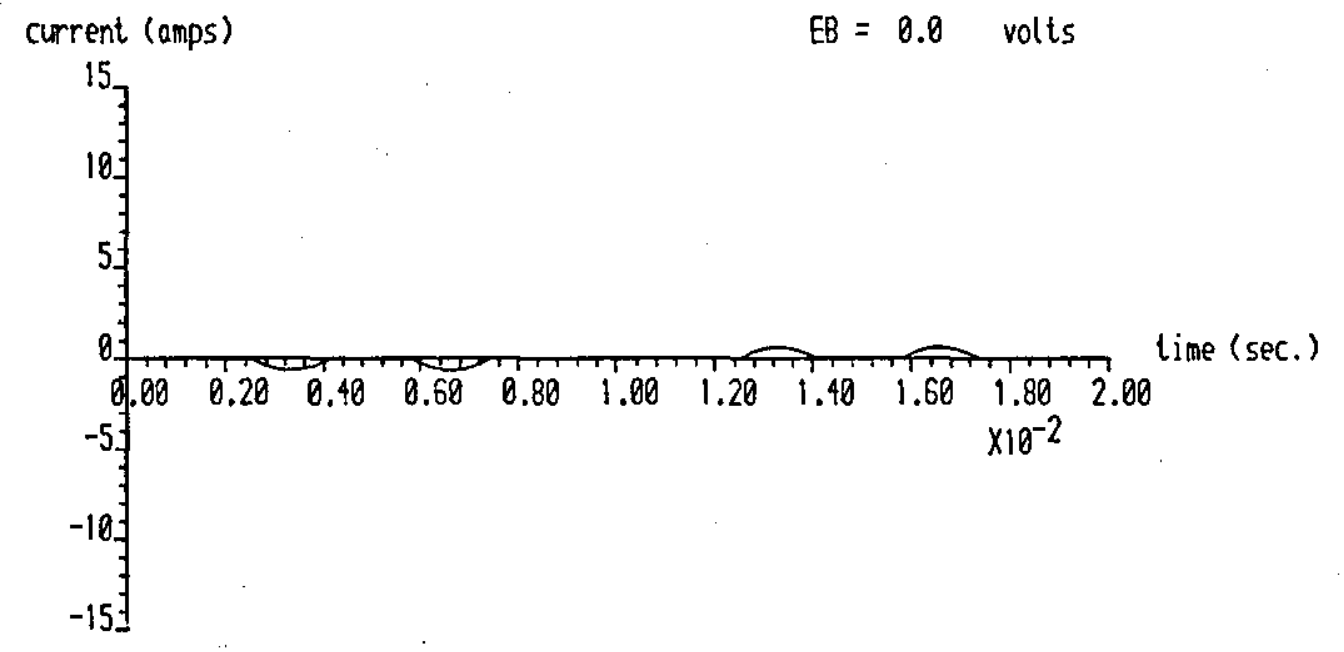

FIG. 6.13a THE BRIDGE SUPPLY LINE CURRENT

$$
I_{\mathrm{d} 1}=0.2 \mathrm{~A} \quad I_{\mathrm{d} 2}=0.2 \mathrm{~A} \quad I_{\mathrm{d}}=0.4 \mathrm{~A}
$$$$
v_{d}=1 \mathrm{~V}
$$

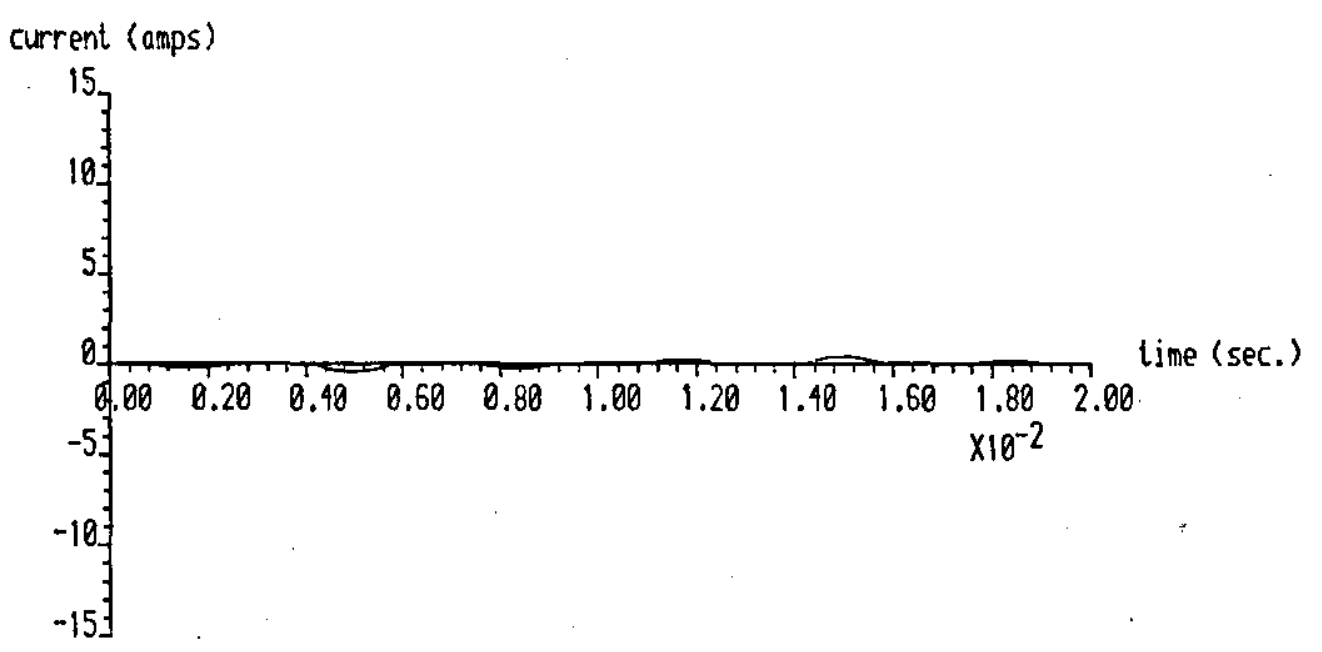

FIG. $6.13 \mathrm{~b}$ THE TERTIARY WINDING CURRENT

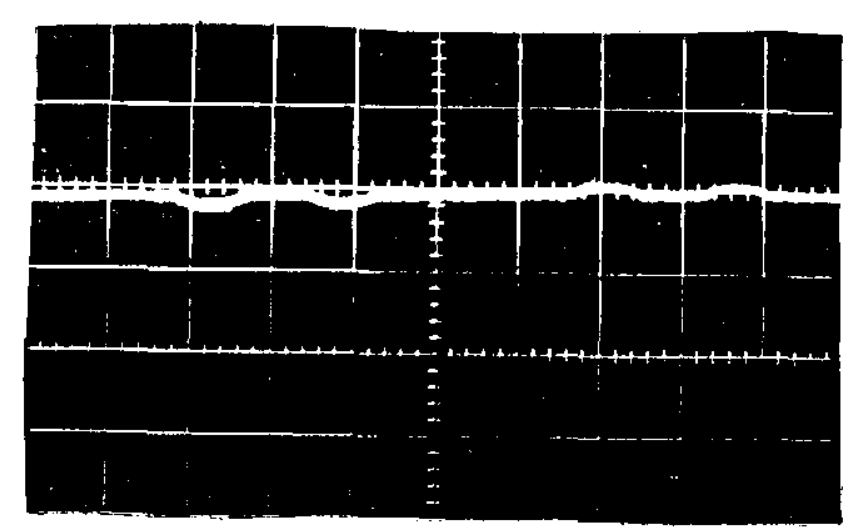

FIG. 6.14a THE BRIDGE STIPPLY IINE CURRFNT $I_{d 1}=0.4 \mathrm{~A} \quad I_{d 2}=0.42 \mathrm{~A} \quad I_{d}=0.82 \mathrm{~A}$ $\mathrm{v}_{\mathrm{d}}=4.2 \mathrm{~V}$

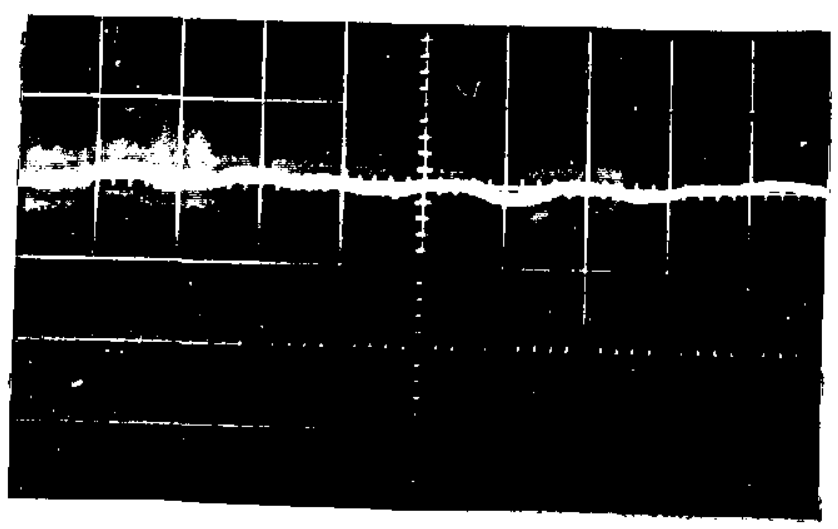

FIG. 6.14b THE TERTIARY WIMDIMG CURRENT THE OSCILLOGRAMS Scale: Horizontal - 2 ms per division Vertical - 5 A per division 
Frequency $=50.0 \mathrm{hz}$ and alpha $=165.0$ deg. RL $=5.10000$ ohms $L=0.017400000 \mathrm{~h}$

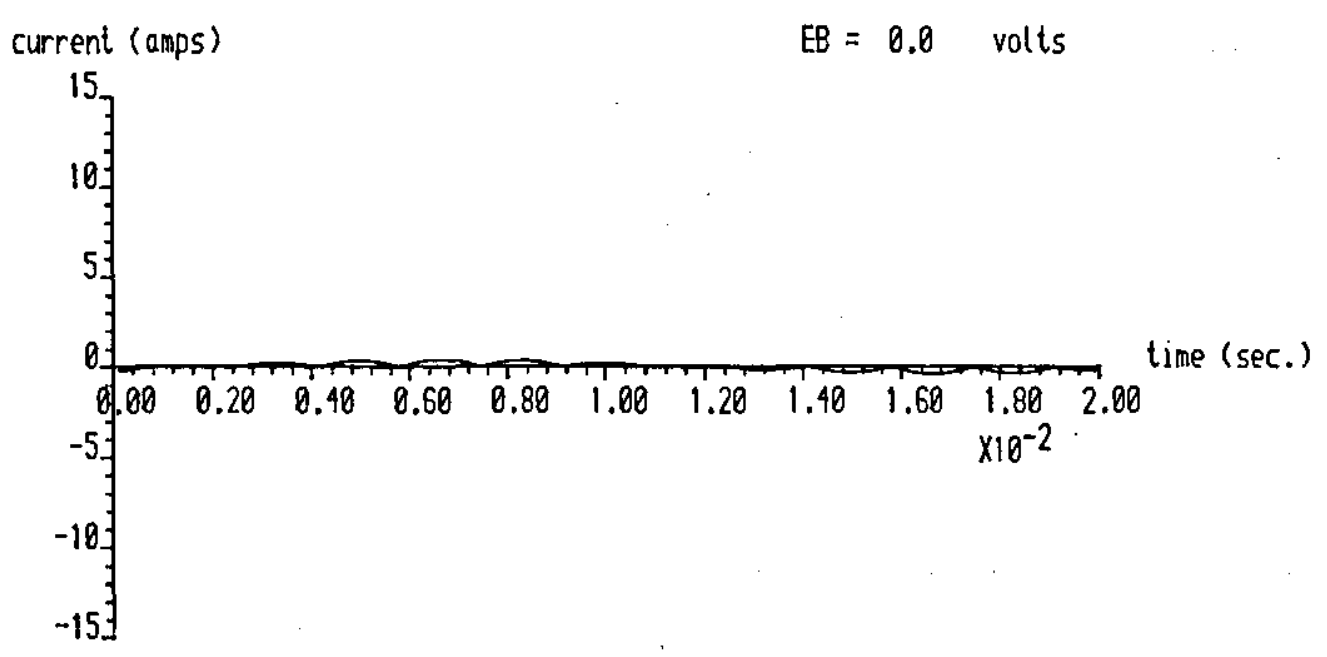

FIG. 6.13c THE SUF'PLY LINE CURRENT

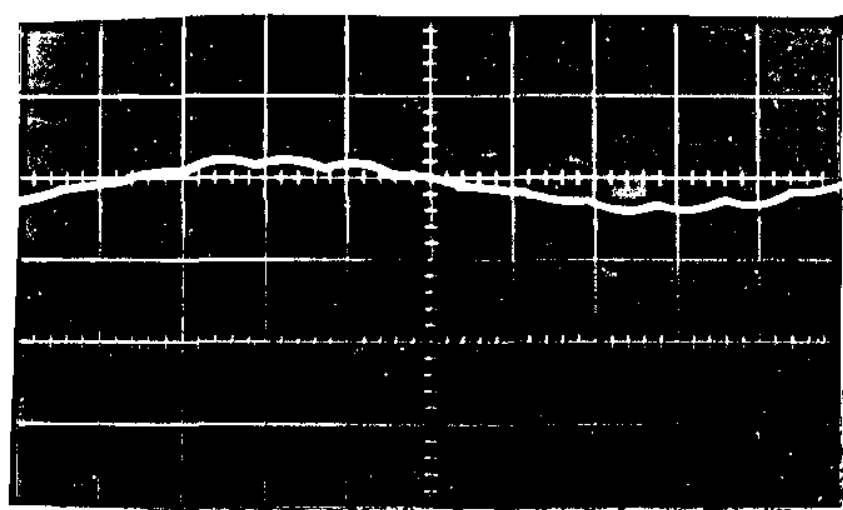

FIG. 6.14c THE SUPSLY IINE CURRENT

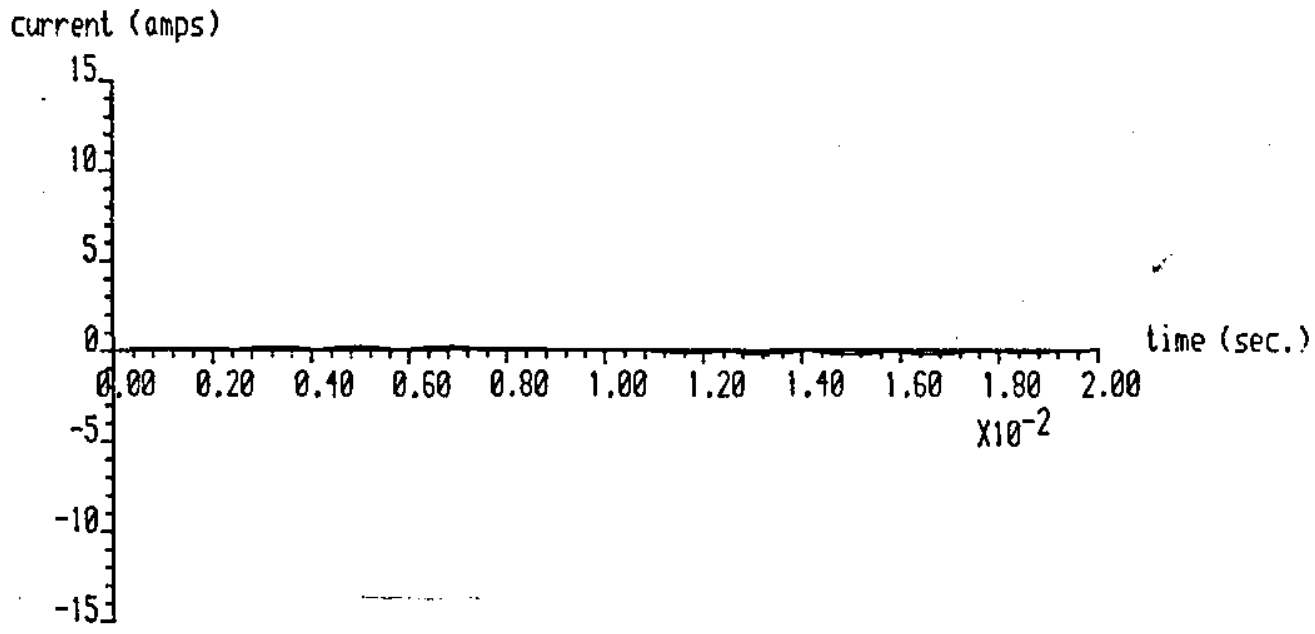

FIG. $6.13 \mathrm{~d}$ THE PRIMARY PHASE CURRENT

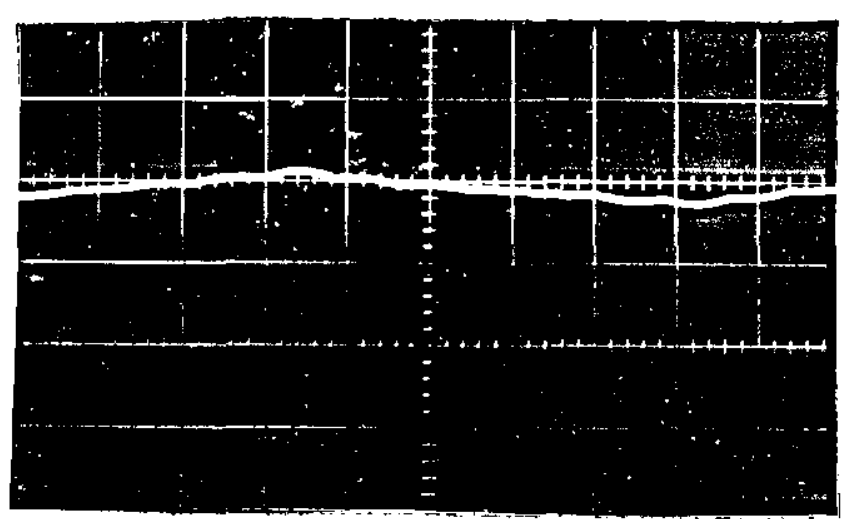

FIG. 6.14d THE PRIMARY PHASE CURPENT THE OSCILLOGRANS Scale: Horizontal - $2 \mathrm{~ms}$ per division Vertical - 5 A per division 
Frequency $=50.0 \mathrm{hz}$ and alpha $=150.0$ deg. RL $=5.10000$ ahms $\mathrm{L}=0.017400000 \mathrm{~h}$

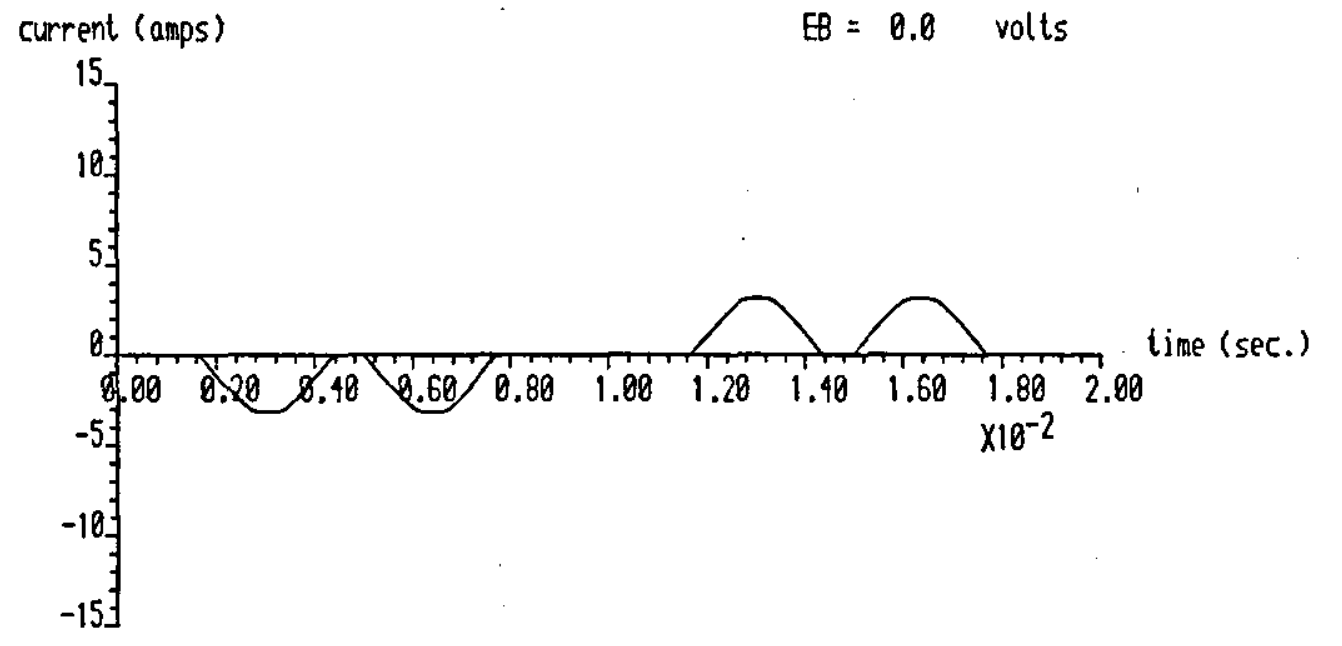

FIG. $6.15 \mathrm{a}$ THE BRIDGE SUPPLY LINE CURRENT

$$
\begin{aligned}
& I_{d 1}=1.61 \mathrm{~A} \quad I_{d 2}=1.61 \mathrm{~A} \quad I_{d}=3.22 \mathrm{~A} \\
& V_{d}=16.3 \mathrm{~V}
\end{aligned}
$$

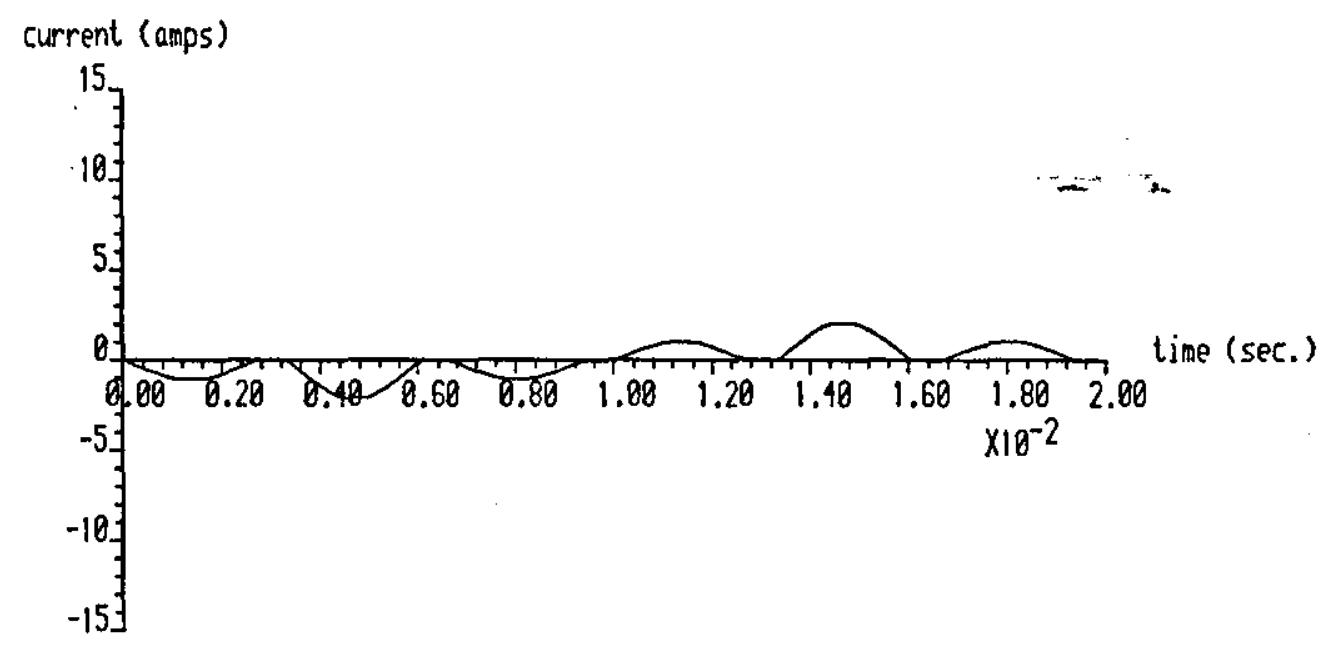

FIG. 6.15b THE TFRTIARY WINDING CURRENT

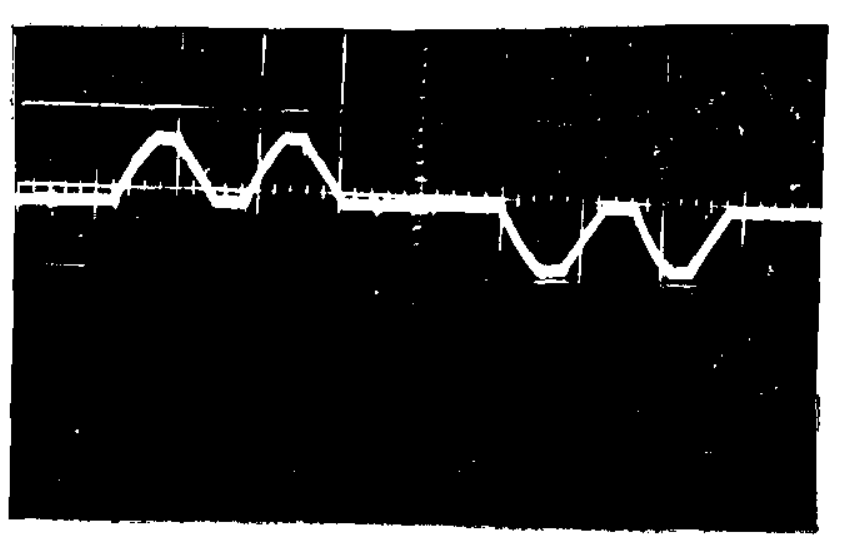

FIG. $6.16 \mathrm{a}$ THE BRIDGE SUPFLY LINE CURRENT

$I_{d 1}=1.8 \mathrm{~A} \quad I_{d 2}=2.15 \mathrm{~A} \quad I_{d}=4.0 \mathrm{~A}$ $\mathrm{v}_{\mathrm{d}}=20.05 \mathrm{~V}$

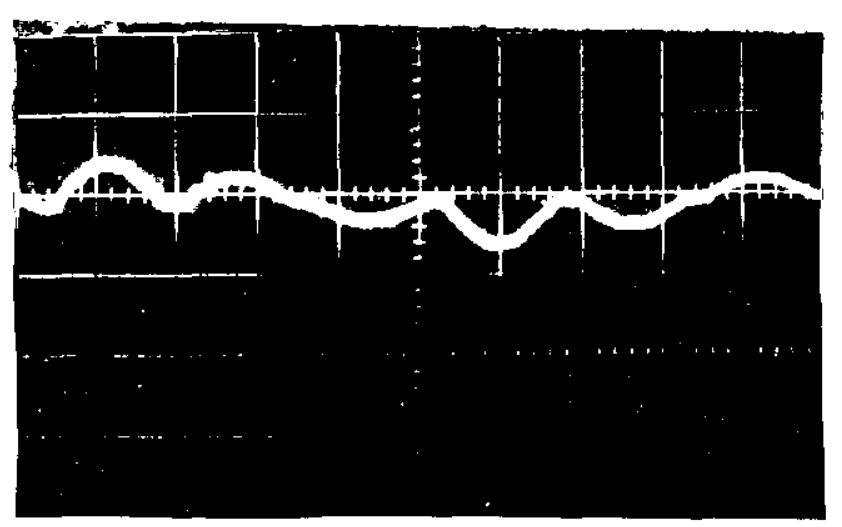

FIG. 6.16b THE TERTIARY WTNDING CURRPNT

THE OSCILOGRAMS

Scale: Horizontal - $2 \mathrm{~ms}$ per division Vertical - 5 A per division 
Frequency $=50.0 \mathrm{hz}$ and alpho $=150.0$ deg. RL $=5.10000$ ohms $L=0.017400000 \mathrm{~h}$

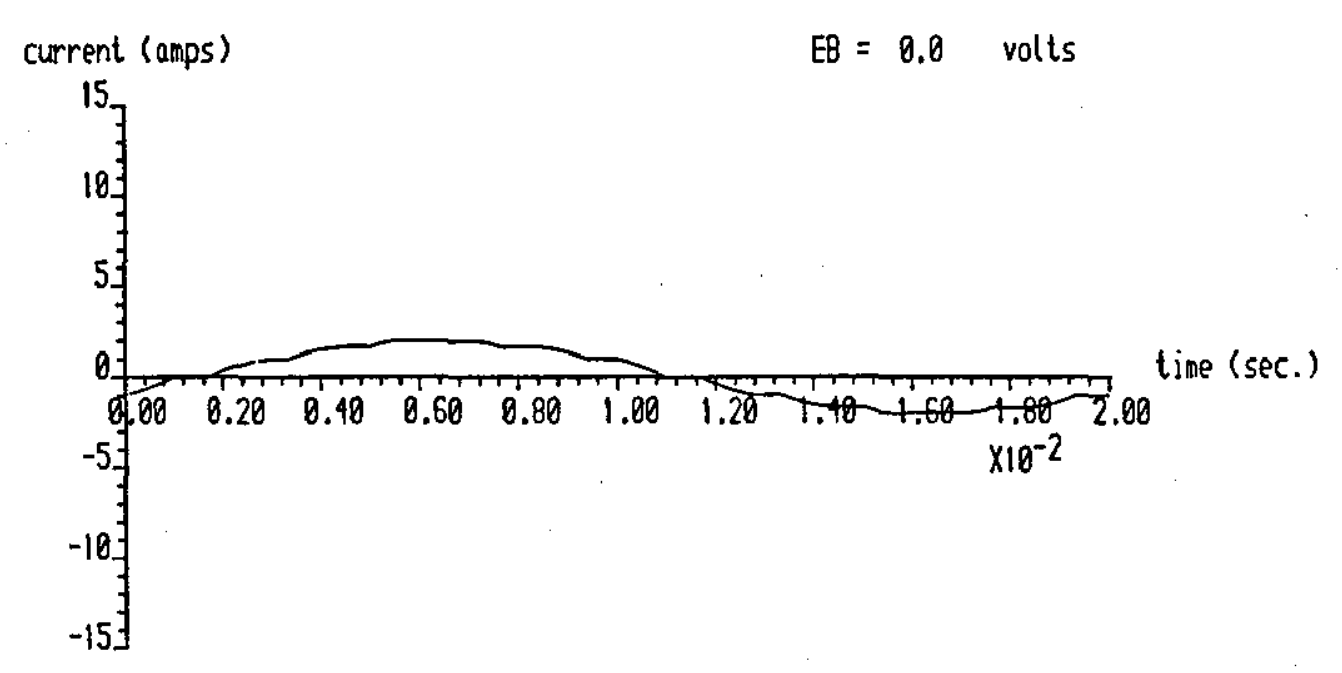

FIG. 6.15c THE SUPPLY LINE CTIRRENT

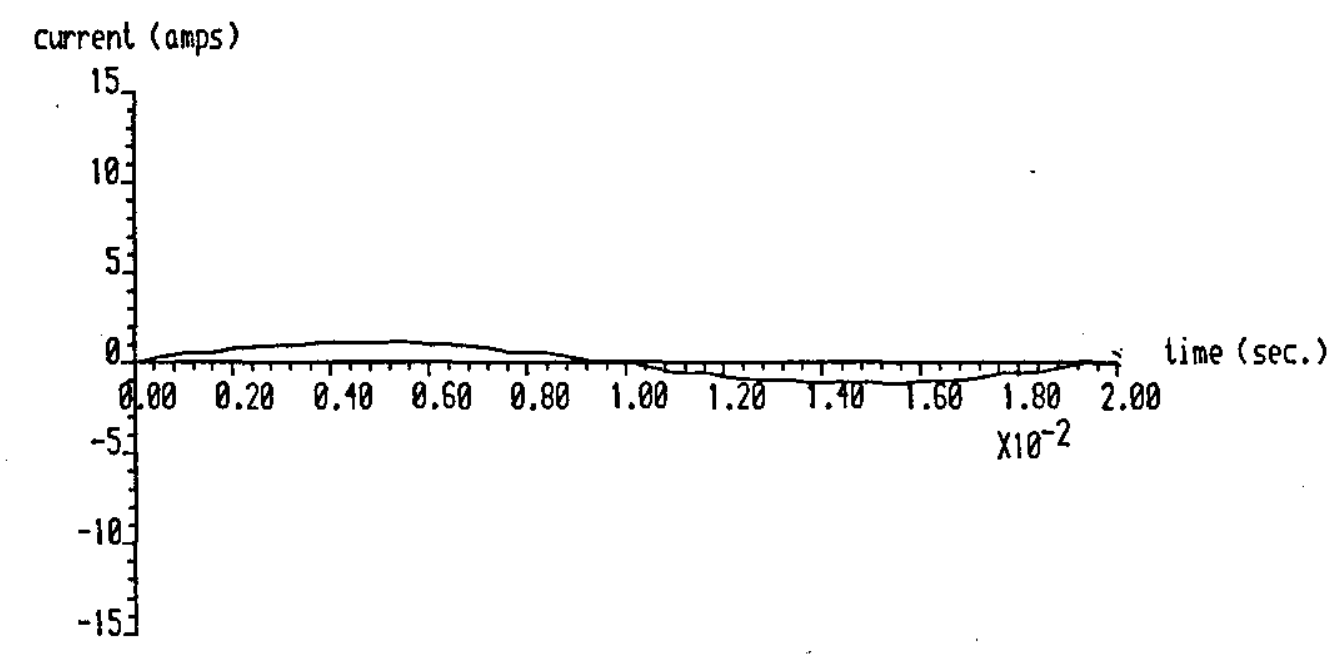

FIG. 6.15d THE PRIMARY PHASE CURRENT

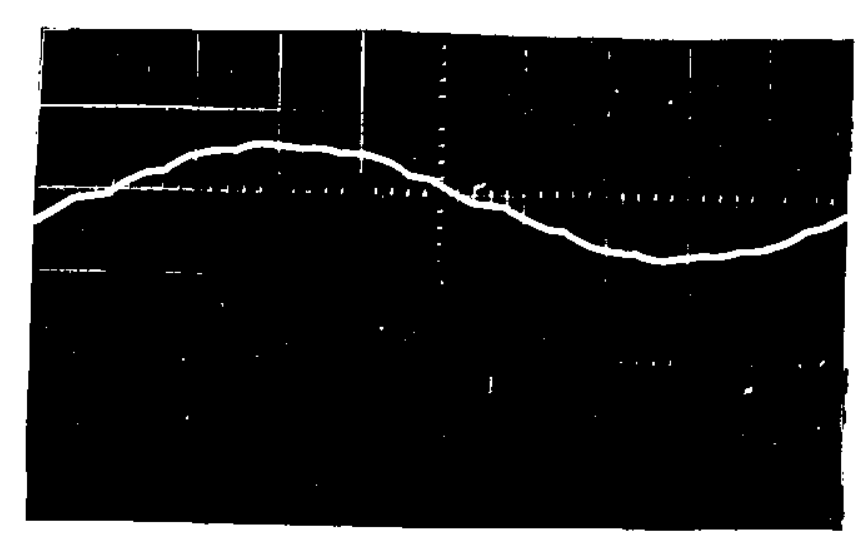

FIG. 6.16c THE SUPPLY LINE CURREMTT

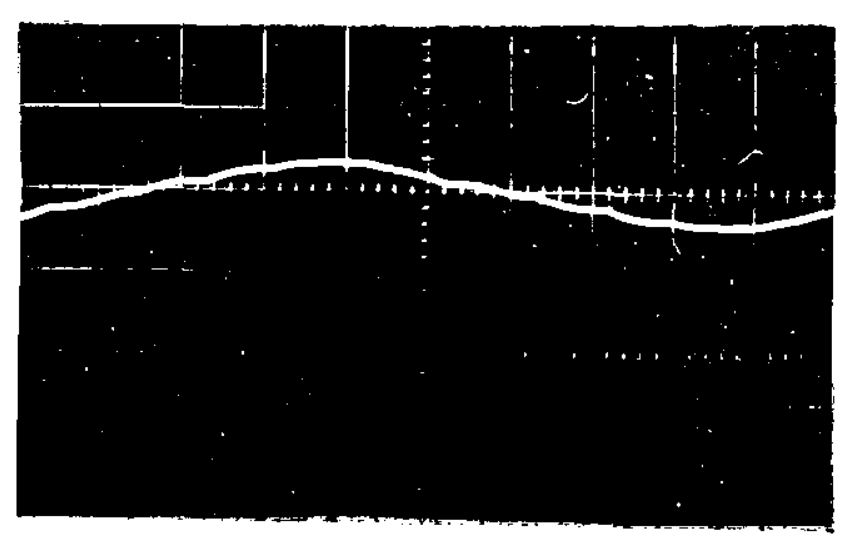

FIG. 6.16d THE PRIMARY PHASE CURRENT

THE OSCILLOGRAMS

Scale: Horizontal - 2 ms per division Vertical - 5 A per division 
Frequency $=50.0 \mathrm{hz}$ and alpha $=128.0$ deg. $\mathrm{RL}=5.10800$ ohms $\mathrm{L}=0.017408080 \mathrm{~h}$

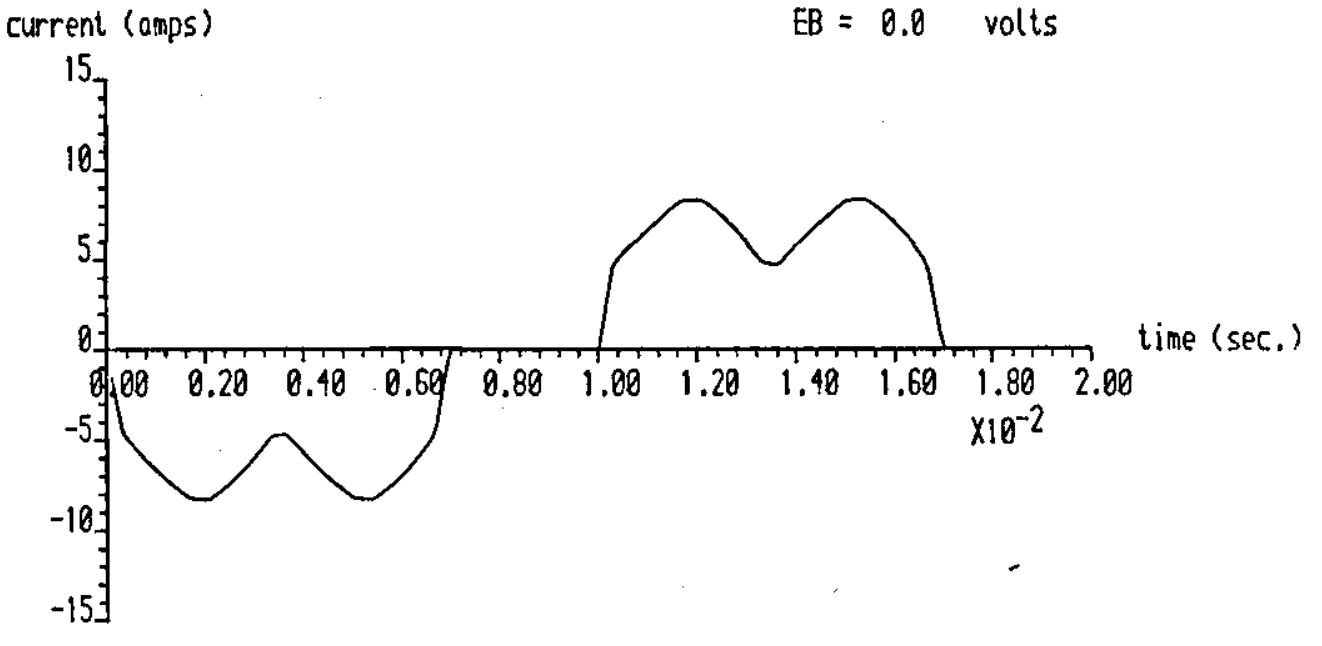

FIG. 6.17a THE BRIDGE SUPPLY LINE CURRENT

$$
\begin{aligned}
& I_{d 1}=6.68 \mathrm{~A} \quad I_{d 2}=7.6 \mathrm{~A} \quad I_{d}=14.28 \mathrm{~A} \\
& V_{d}=72.8 \mathrm{~V}
\end{aligned}
$$

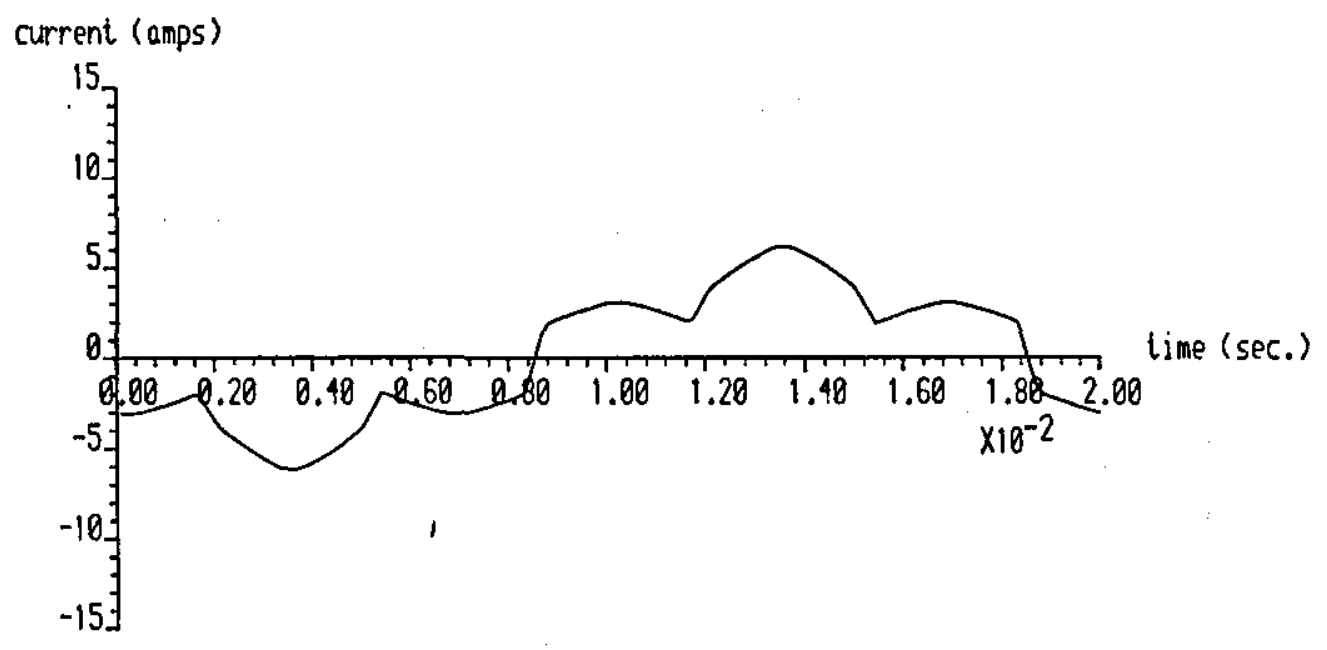

FIG. $6.17 \mathrm{~b}$ THE TERTIARY WINDING CURRENT

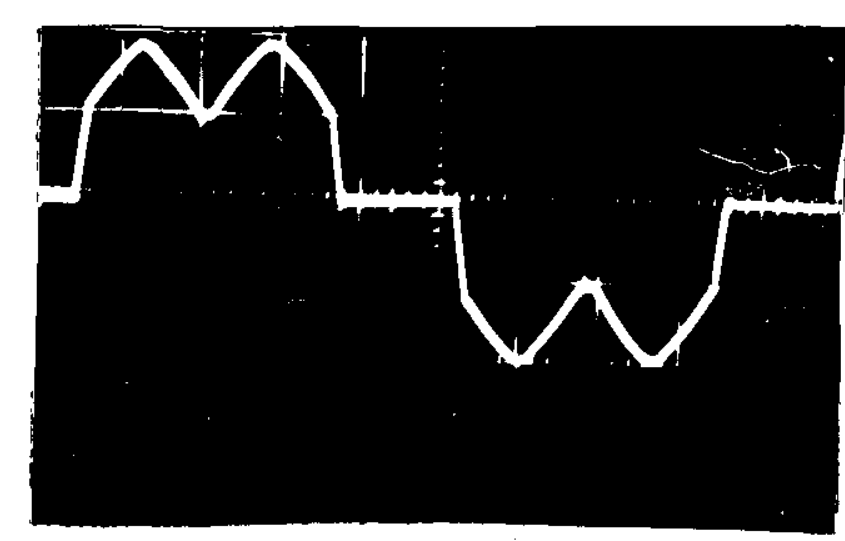

FIG. 6.18a THE BRIDGF SUPIJY LINE CURRENT' $I_{d 1}=7.15 \mathrm{~A} \quad I_{d 2}=7.2 \mathrm{~A} \quad I_{d}=14.5 \mathrm{~A}$ $\mathrm{v}_{\mathrm{d}}=74 \mathrm{~V}$

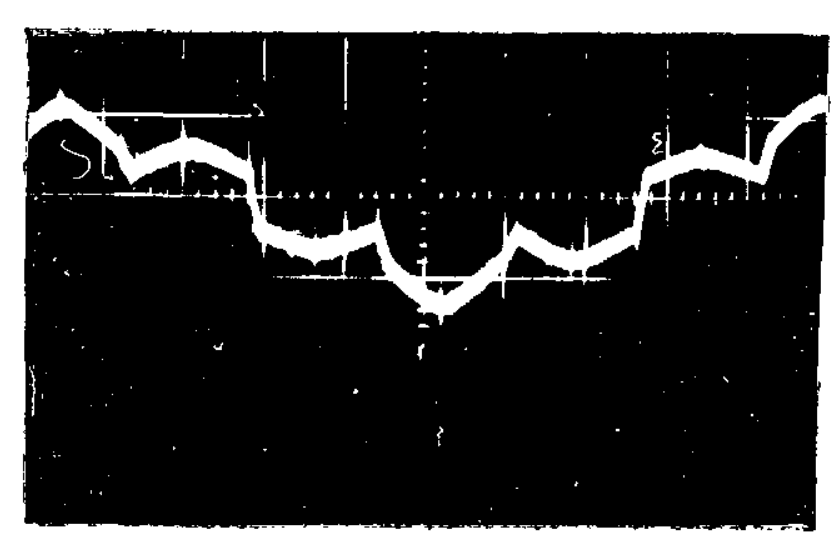

FIG. 6.18b THF TERTIARY WINDING CURRENT

THE OSCILLOGRAMS

Scale: Horizontal - $2 \mathrm{~ms}$ per division Vertical - 5 A per division 
Frequency $=50.0 \mathrm{hz}$ and alpha $=120.8 \mathrm{deg} . \mathrm{RL}=5.10000$ ohms $L=0.017400800 \mathrm{~h}$

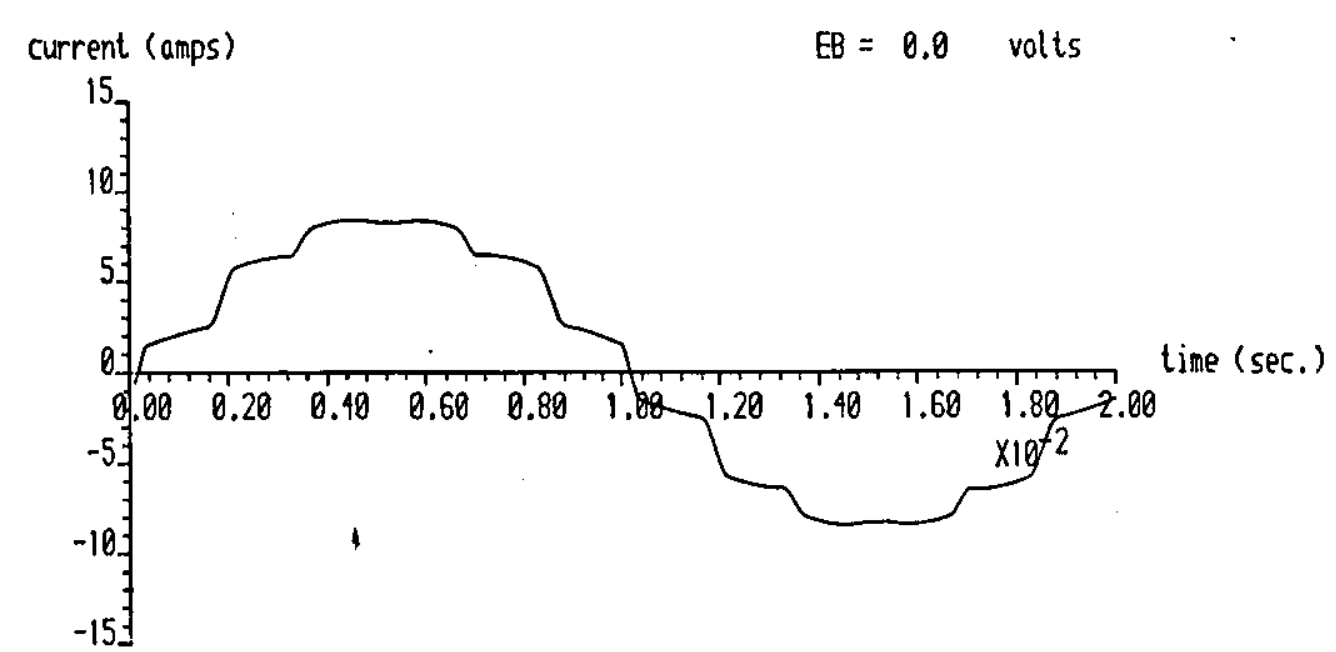

FIG. 6.17C THE SUPPLY LINE CURRENT

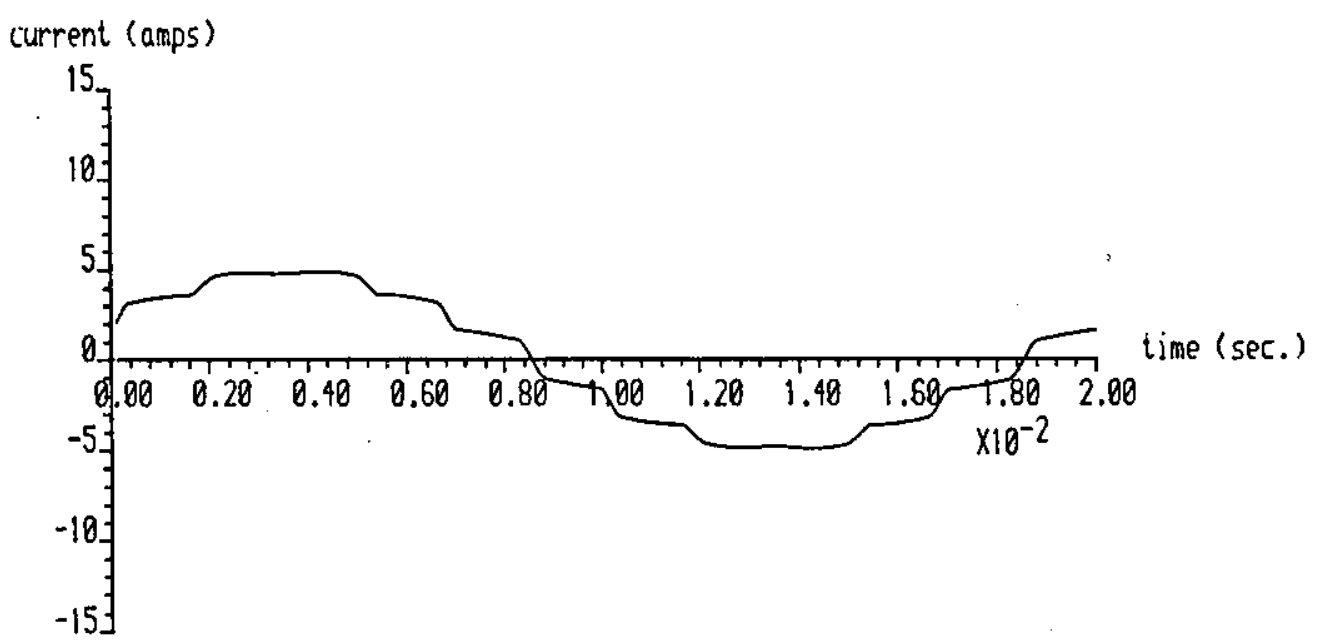

FIG. 6.17d THE PRIMARY PHASE CURRENT

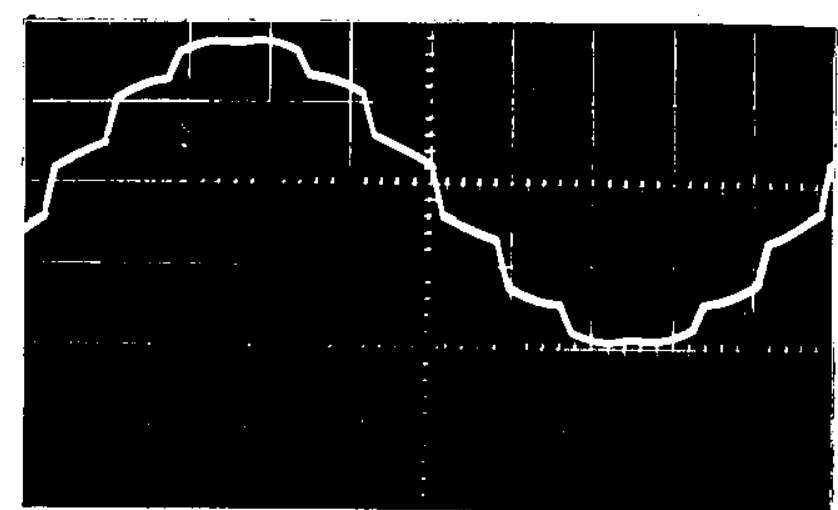

FIG. 6.18C THE SUPYLY IIME CURRENT

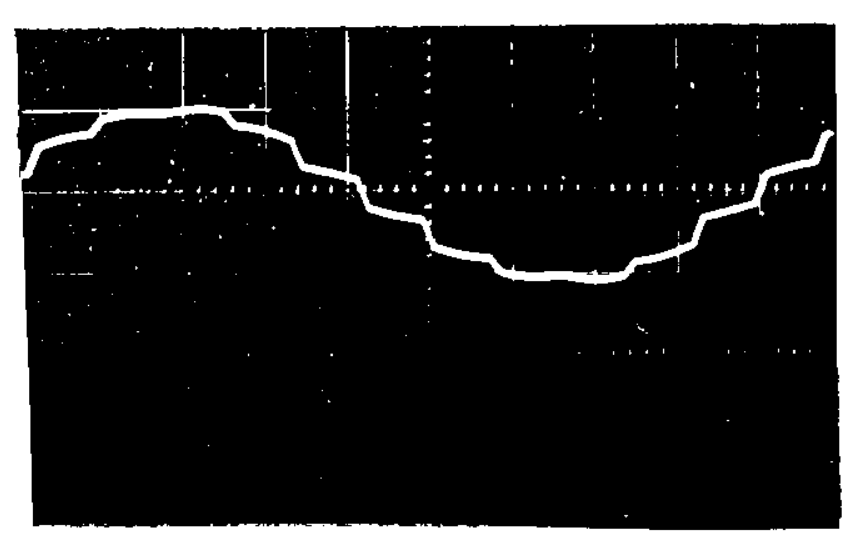

FIG. 6.18d THE PRIMARY FHASE CURRENT

THE OSCILLOGRAMS

Scale: Horizontal - 2 ms per division Vertical - 5 A per division 
Frequency $=50.0 \mathrm{hz}$ and alpha $=60.0$ deg. RL $=5.10000$ ohms $\mathrm{L}=0.017408080 \mathrm{~h}$

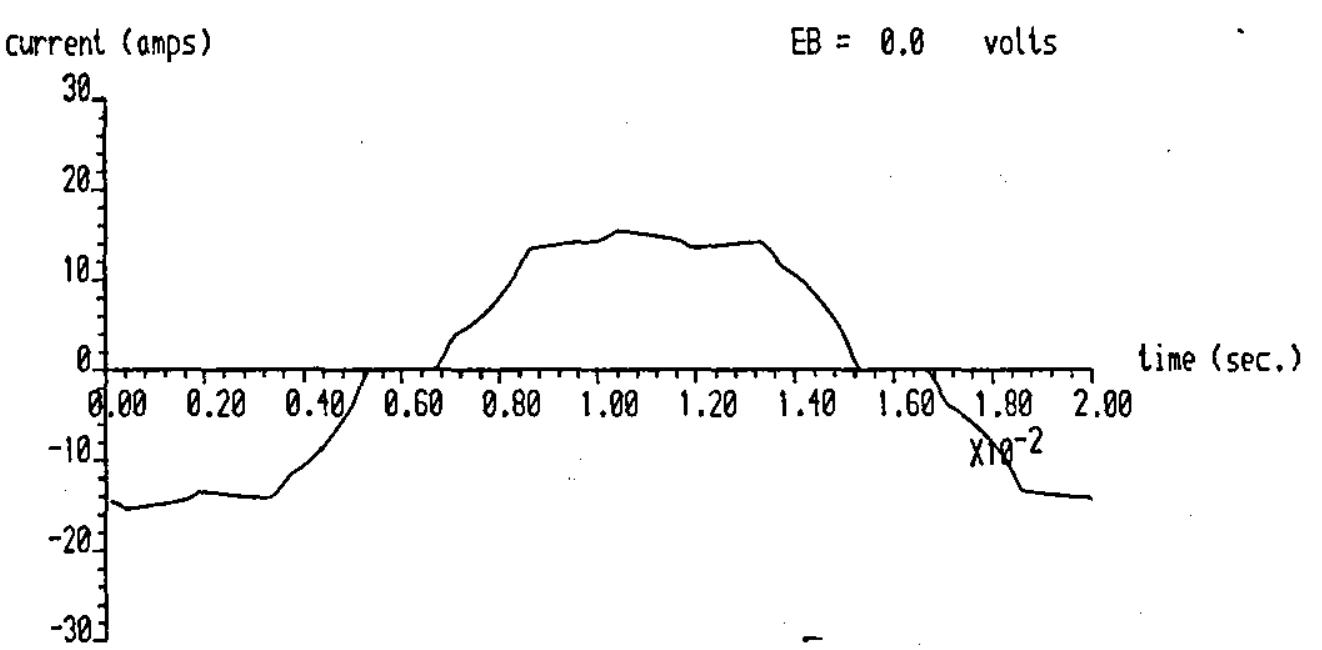

FIG. 6.19a THE BRIDGE SUIPLY IINE CURRENT

$I_{\mathrm{d} 1}=14.41 \mathrm{~A} \quad I_{\mathrm{d} 2}=14.57 \mathrm{~A} \quad I_{\mathrm{d}}=28.98 \mathrm{~A}$

$\mathrm{v}_{\mathrm{d}}=147.3 \mathrm{~V}$

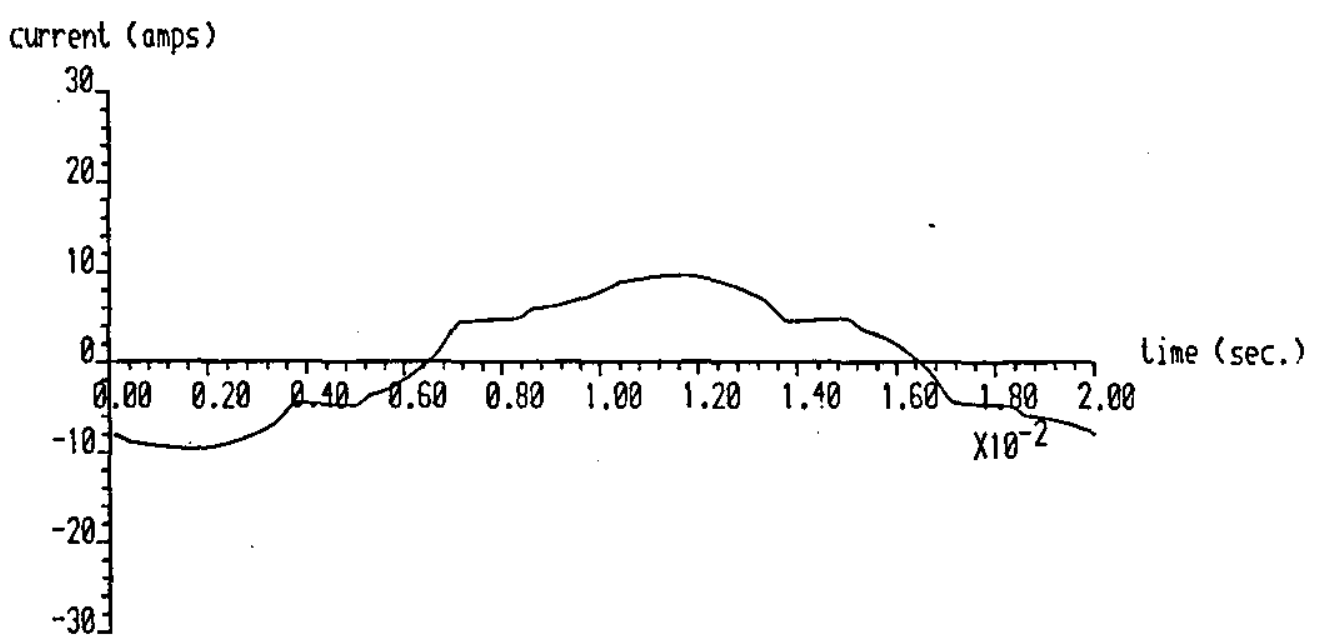

FIG: $6.19 \mathrm{~b}$ THE TERTIARY WINDING CURRENT

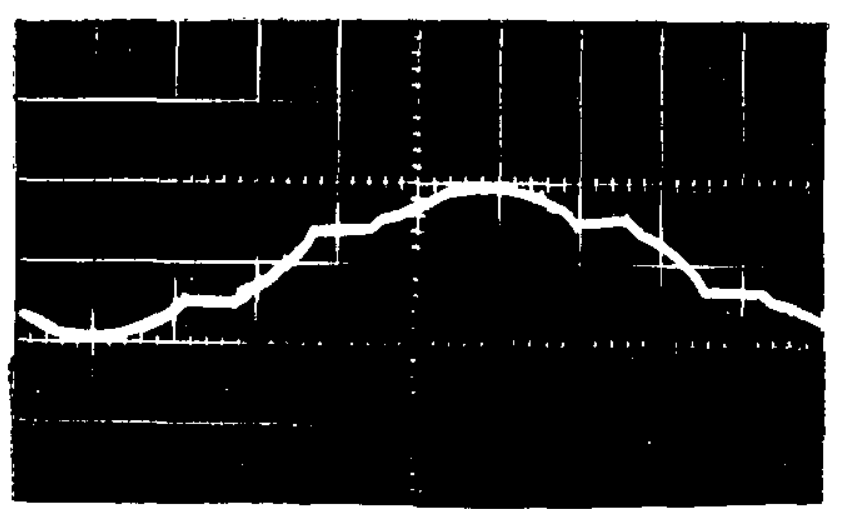

FIG. 6.20b THE TERTIARY WINDING CURRETT

THE OSCILLOGRAMS

Scale: Horizontal - 2 ms per division

Vertical - 10 A per division 
Frequency $=50.0 \mathrm{hz}$ and alpha $=60.8 \mathrm{deg}$. RL $=5.10080$ ohms $\mathrm{L}=0.017408090 \mathrm{~h}$

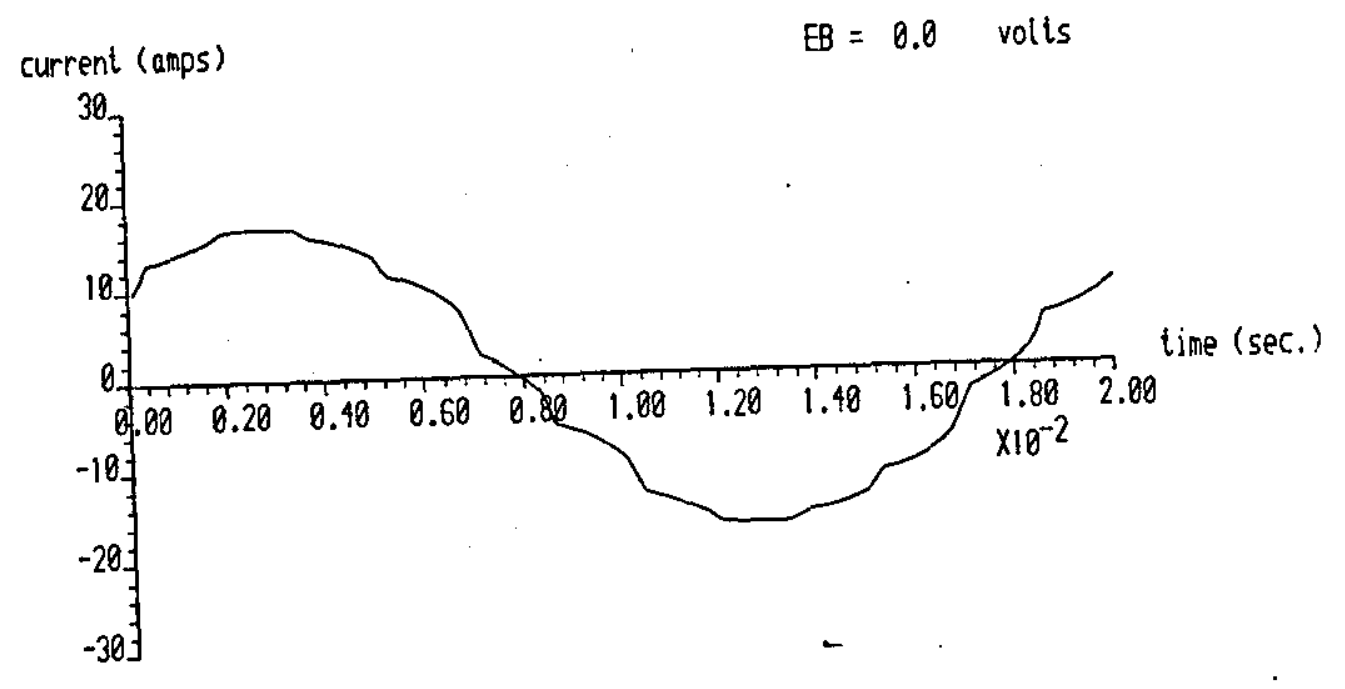

FTG. 6.19c THE STIPPTY LINE CURRENT

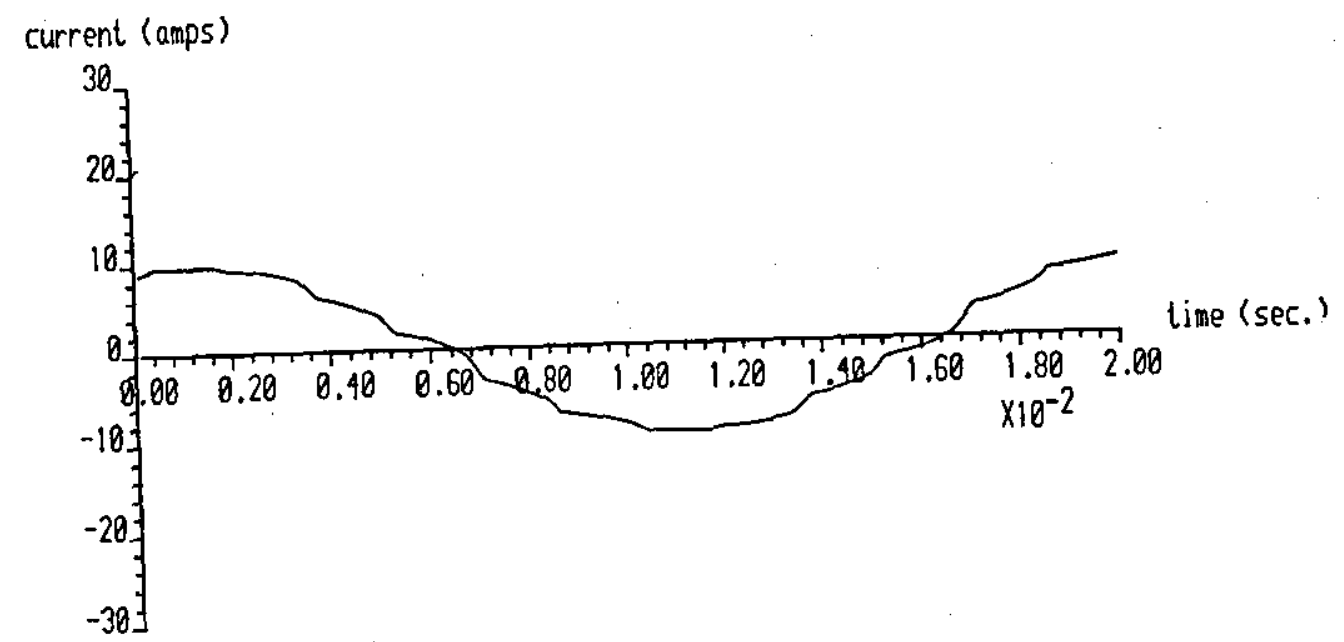

FTG. 6.19d THE PRTMARY PIIASE CTRREIT

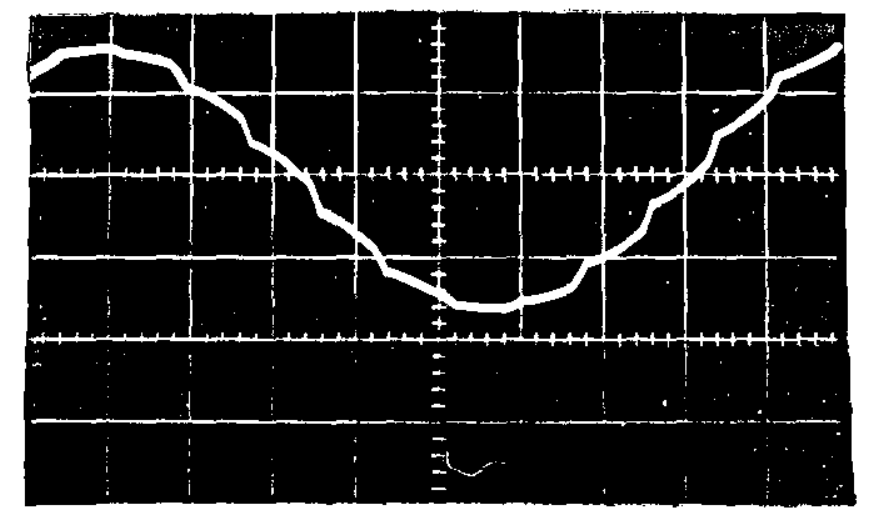

FIG. $6.20 \mathrm{c}$ THE SUPJIY IINE CUTRRENT

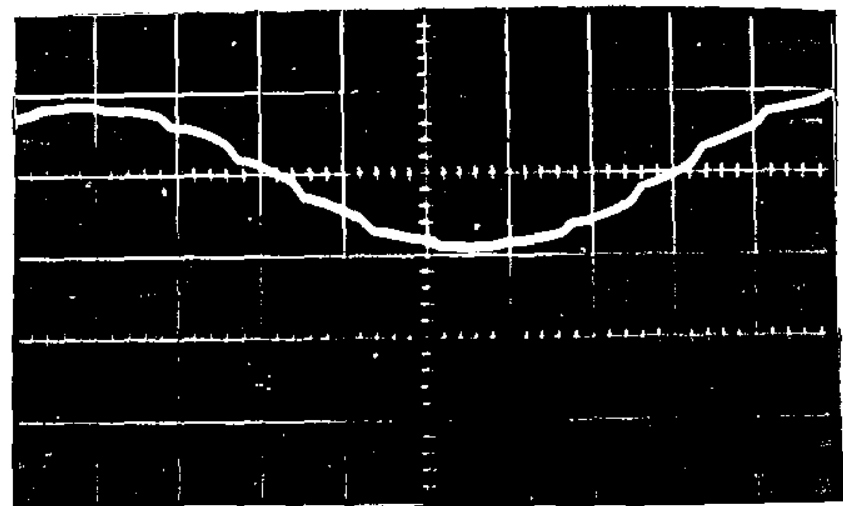

FIG. 6.20d THE FRIMARY FHASE CIRREYT THE OSCILIOGRAMS

Scale: Horizontal - 2 ms per division Vertical - 10 A per division 
values indicate acceptable agreement.

Generally, within the limits of unbalanced impedances and bridge supply voltages obtainable when setting up the transformers, the predicted and measured results correspond very well indeed. The setting of the $30^{\circ}$ phase shifting transformer is critical, and balance between the bridges is disturbed by only a very few degrees deviation from the nominal $30^{\circ}$. This shows itself in differences of conduction angle and mean values of the bridge currents. The initial setting up of this $30^{\circ}$ displacement has been performed as accurately as possible on very light load with each bxidge set up separately to operate identically for $\alpha=75^{\circ}$ on the master firing delay controllex. Nevertheless, the possibility of a small error in the $30^{\circ}$ displacement for the on-load operating conditions cannot be ruled out, especially as some small unbalance of firing between the thyristors within each bridge is inevitable.

As is to be expected, the waveforms become much closer to sinusoidal on the primary side when the two secondary winding currents are added. Naturally, this is most evident at the higher operating modes.

To verify the harmonic contents of the current waveforms, for experimental convenience no back emf is included and the firing delay angles have been set at $165^{\circ}, 150^{\circ}, 120^{\circ}$ and $60^{\circ}$ for the four modes of operation. The waveforms are demonstrated in Figs. 6.13 to 6.20. Predicted components include the fundamental and up to the 20th harmonic. The harmonics are expressed as a ratio relative to the fundamental. The measurements have been made of the supply currents to both bridges and in the red and blue phases. The harmonic 
currents in the delta tertiary windings are measured for all the three phases to gauge the effect of the small transformer winding impedance and voltage unbalance.

The results of measured harmonic contents of the current waveforms are provided in Tables $6.4,6.5,6.6$ and 6.7 . The first of these gives a comparison of the harmonics in the supply currents to the bridges, as well as the values in the two phases to each bridge which show most discrepancy. In Table 6.5 , the harmonic contents of the currents for all the three phases of the delta tertiary are shown.

The measurements give an acceptable correlation of harmonics between phases and between the two bridges except for Table 6.5 with a firing delay angle of $165^{\circ}$. Here the per unit harmonic currents with respect to the fundamental in phase $C$ are greater than those in the other phases by a factor of about 10. This is due to a small sinusoidal circulating current round the delta loop which is additive or subtractive to some degree with each of the $120^{\circ}$ - displaced fundamental current components supplying the load. Evidently, it is subtractive in phase $c$, giving a low fundamental and resulting in high per unit harmonic values. (Fundamental phase current components of $0.28 \mathrm{~A}, 0.25 \mathrm{~A}$ and $0.02 \mathrm{~A}$ respectively in the three phases are presentl. The circulating current is caused by a small phase voltage unbalance round the delta, and it is significant that it only manifests itself on very low load.

Tables 6.6 and 6.7 provide the computed harmonics and allow comparison with the experimental results in Tables 6.4 and 6.5 . For the same firing delay angles, it is evident that although correlation 


\begin{tabular}{|ll|l|l|l|l|l|l|}
\hline$\alpha$ & Phase & 5 th & 7 th & 11 th & 13 th & 17 th & 19 th \\
\hline $165^{\circ}$ & Red & 0.863 & 0.685 & 0.508 & 0.355 & 0.173 & 0.069 \\
& Blue & 0.795 & 0.614 & 0.455 & 0.318 & 0.168 & 0.082 \\
$150^{\circ}$ & Red & 0.63 & 0.46 & 0.08 & 0.031 & 0.066 & 0.046 \\
& Blue & 0.595 & 0.405 & 0.081 & 0.030 & 0.073 & 0.041 \\
$120^{\circ}$ & Red & 0.26 & 0.022 & 0.056 & 0.034 & 0.047 & 0.023 \\
& Blue & 0.24 & 0.021 & 0.06 & 0.036 & 0.037 & 0.025 \\
$60^{\circ}$ & Red & 0.102 & 0.05 & 0.02 & 0.024 & 0.02 & 0.017 \\
& Blue & 0.095 & 0.046 & 0.023 & 0.021 & 0.017 & 0.016 \\
\hline
\end{tabular}

(a)
\begin{tabular}{|ll|l|l|l|l|l|l|}
\hline$\alpha$ & on the stax side (Bridge 1) \\
\hline $165^{\circ}$ & Phase & 5 th & 7 th & 11 th & 13 th & 17 th & 19 th \\
& Blue & 0.897 & 0.795 & 0.513 & 0.308 & 0.154 & 0.072 \\
$150^{\circ}$ & Red & 0.792 & 0.625 & 0.438 & 0.30 & 0.104 & 0.05 \\
& Blue & 0.59 & 0.34 & 0.032 & 0.063 & 0.066 & 0.024 \\
$120^{\circ}$ & Red & 0.26 & 0.023 & 0.06 & 0.037 & 0.044 & 0.024 \\
- & Blue & 0.235 & 0.022 & 0.063 & 0.038 & 0.048 & 0.027 \\
$60^{\circ}$ & Red & 0.102 & 0.047 & 0.026 & 0.021 & 0.021 & 0.017 \\
& Blue & 0.09 & 0.045 & 0.02 & 0.02 & 0.017 & 0.015 \\
\hline
\end{tabular}

(b) on the delta side (Bridge 2)

TABLE 6.4 BRIDGE SUPPLY LINE CURRENT HARMONICS (EXPERIMENTAL) AS A FRACTION OF THE FUNDAMENTAL 


\begin{tabular}{|l|c|c|l|l|l|l|l|}
\hline & Phase & 5 th & 7 th & 11 th & 13 th & 17 th & 19 th \\
\hline \multirow{3}{*}{$165^{\circ}$} & A & 0.347 & 0.333 & 0.181 & 0.10 & 0.044 & 0.021 \\
& B & 0.549 & 0.431 & 0.275 & 0.204 & 0.069 & 0.029 \\
& C & 5.25 & 4.0 & 3.50 & 2.0 & 0.825 & 0.375 \\
\hline \multirow{3}{*}{$150^{\circ}$} & A & 0.60 & 0.36 & 0.028 & 0.068 & 0.06 & 0.04 \\
& B & 0.667 & 0.383 & 0.028 & 0.071 & 0.058 & 0.038 \\
& C & 0.538 & 0.292 & 0.026 & 0.054 & 0.058 & 0.028 \\
\hline \multirow{3}{*}{$120^{\circ}$} & A & 0.27 & 0.028 & 0.058 & 0.038 & 0.046 & 0.024 \\
& B & 0.29 & 0.023 & 0.058 & 0.036 & 0.046 & 0.024 \\
& C & 0.264 & 0.023 & 0.06 & 0.035 & 0.044 & 0.023 \\
\hline \multirow{2}{*}{$60^{\circ}$} & A & 0.10 & 0.052 & 0.02 & 0.018 & 0.016 & 0.015 \\
& B & 0.092 & 0.054 & 0.022 & 0.023 & 0.018 & 0.016 \\
& C & 0.091 & 0.045 & 0.02 & 0.02 & 0.018 & 0.017 \\
\hline
\end{tabular}

TABLE 6.5 DELTA TERTIARY WINDING CURRENT HARMONICS (EXPERIMENTAL) AS A FRACTION OF THE FUNDAMENTAL 


\begin{tabular}{|l|l|l|l|l|l|l|}
\hline$\alpha$ & 5 th & 7 th & 11 th & 13 th & 17 th & 19 th \\
\hline $165^{\circ}$ & 0.86 & 0.735 & 0.43 & 0.28 & 0.046 & 0.031 \\
$167^{\circ} *$ & 0.892 & 0.793 & 0.541 & 0.406 & 0.161 & 0.065 \\
$150^{\circ}$ & 0.64 & 0.38 & 0.009 & 0.056 & 0.019 & 0.014 \\
$154^{\circ} *$ & 0.696 & 0.462 & 0.06 & 0.046 & 0.073 & 0.037 \\
$120^{\circ}$ & 0.29 & 0.03 & 0.069 & 0.04 & 0.039 & 0.026 \\
$122^{\circ} *$ & 0.301 & 0.021 & 0.070 & 0.038 & 0.041 & 0.025 \\
$60^{\circ}$ & 0.085 & 0.049 & 0.036 & 0.030 & 0.006 & 0.008 \\
$58^{\circ} *$ & 0.080 & 0.047 & 0.038 & 0.030 & 0.0 & 0.007 \\
\hline
\end{tabular}

TABLE 6.6 BRIDGE SUPPLY LINE CURRENT HARMONICS (COMPUTED) AS A FRACTION OF THE FUNDAMENTAL

\begin{tabular}{|l|l|l|l|l|l|l|}
\hline$\alpha$ & 5 th & 7 th & 11 th & 13 th & 17 th & 19 th \\
\hline $165^{\circ}$ & 0.859 & 0.732 & 0.427 & 0.277 & 0.041 & 0.034 \\
$167^{\circ} *$ & 0.891 & 0.790 & 0.536 & 0.40 & 0.155 & 0.060 \\
$150^{\circ}$ & 0.637 & 0.377 & 0.0 & 0.057 & 0.017 & 0.016 \\
$154^{\circ} *$ & 0.692 & 0.456 & 0.054 & 0.051 & 0.072 & 0.036 \\
$120^{\circ}$ & 0.278 & 0.040 & 0.064 & 0.044 & 0.035 & 0.024 \\
$122^{\circ} *$ & 0.287 & 0.032 & 0.065 & 0.043 & 0.038 & 0.024 \\
$60^{\circ}$ & 0.098 & 0.048 & 0.033 & 0.031 & 0.007 & 0.0 \\
$58^{\circ} *$ & 0.087 & 0.041 & 0.037 & 0.032 & 0.007 & 0.0 \\
\hline
\end{tabular}

TABLE 6.7 DELTA TERTIARY WINDING CURRENT HARMONICS (COMPUTED) AS A FRACTION OF THE FUNDAMENTAL. 
is acceptable at the lower orders, it is not so for the higher orders except at $\alpha=120^{\circ}$, that is, Mode 3. This may be accounted for by the slight unbalance in the experimental equipment and inaccuracy in setting the firing delay angle $\alpha$. It has been mentioned that the slight unbalances have brought about the difference in the conducting period as observed from the oscillograms and the predicted waveforms. Therefore results have been re-computed with the firing delay adjusted to provide the same conduction period as obtained experimentally. These results are given in Tables 6.6 and 6.7 and marked *, and clearly show the improvement in the higher order harmonics when compared with the related results in Table 6.4. The variation of the firing delay angle required is small, being between $2^{\circ}$ and $4^{\circ}$, illustrating the sensitivity of the harmonic levels to firing delay. A point worth mentioning here is that within the device conduction interval, the commutation periods in Modes 2, 3 and 4 do not correlate perfectly with predictions. Results are given in the next section. In summary, the sources of discrepancy in the results obtained experimentally compared with those computed are:
a) slight bridge ac supply voltage unbalance;
b) small transformer phase impedances unbalance;
c) small firing delay unbalance between thyristors within each bridge and between bridges;
d) inaccuracy in setting the firing delay angle;
e) normal measurement inaccuracy inherent with the meters and CRO.

The computer program assumes perfect balance relating to items (a) to (d), average circuit values being used. 


\subsection{Operating charactexistics}

It can be accepted from the results of the last section that the computer program is valid and able to be used confidently to obtain various desirable operating characteristics. Now data relating to a 12-pulse parallel bridge system, operating with or without an interbridge reactor, can be used in the program for prediction of the performance, as required, in terms of dc output voltage and current, critical firing delay angles and commutation angles, as well as the ac side current waveforms.

Fig. 6.21 shows the experimental briage system output characteristic. This exhibits the expected fall of the dc output voltage as the load current is increased by reducing the load resistance.

Other characteristics plotted from the computer program are shown in Fig. 6.22 to Fig. 6.28. Hexe the firing delay angle is the independent varfable parameter plotted with the following dependent variables:

(a) Device conduction interval (anglel;

(b) Interbridge commutation angle;

(c) Interphase commutation angle;

(d) Interphase commutation overlap angle;

(e) The ac line current harmonics.

All the above are measured in degrees with the exception of the harmonics which are in per unit relative to the fundamental. The operating characteristics are plotted for both bridgés as noted by the legend. This is to observe if any. differences exist between the two bridges although for a balanced system it is to be expected that the results would be the same. For the purpose of some limited 


\title{
FIG. 6.21 LOAD CHARACTERISTICS
}

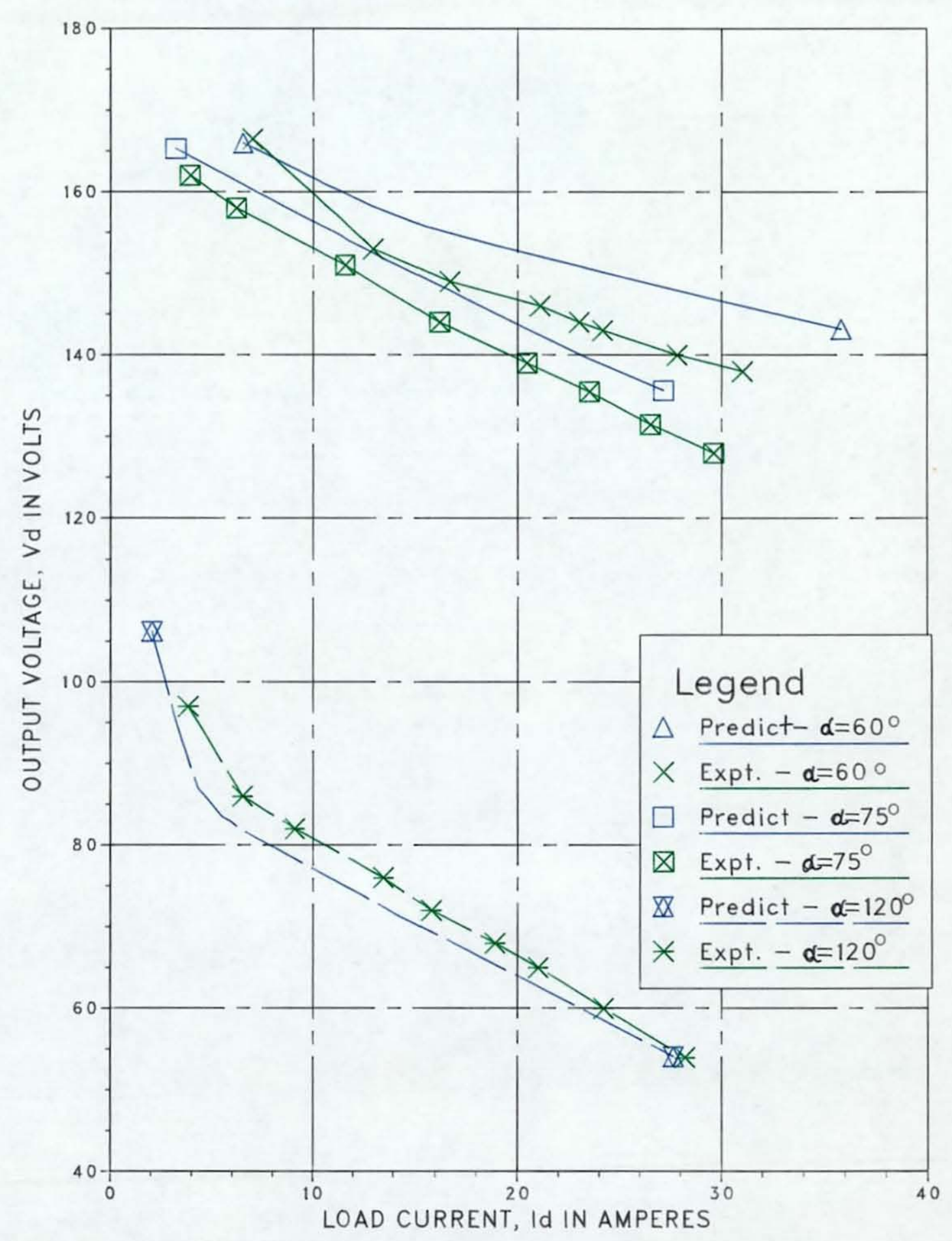

\author{
Load data \\ $\mathrm{L}=0.0174 \mathrm{HEd}=0$ Volt
}




\section{FIG. 6.22 DEVICE CONDUCTION INTERVAL CHARACTERISTICS}

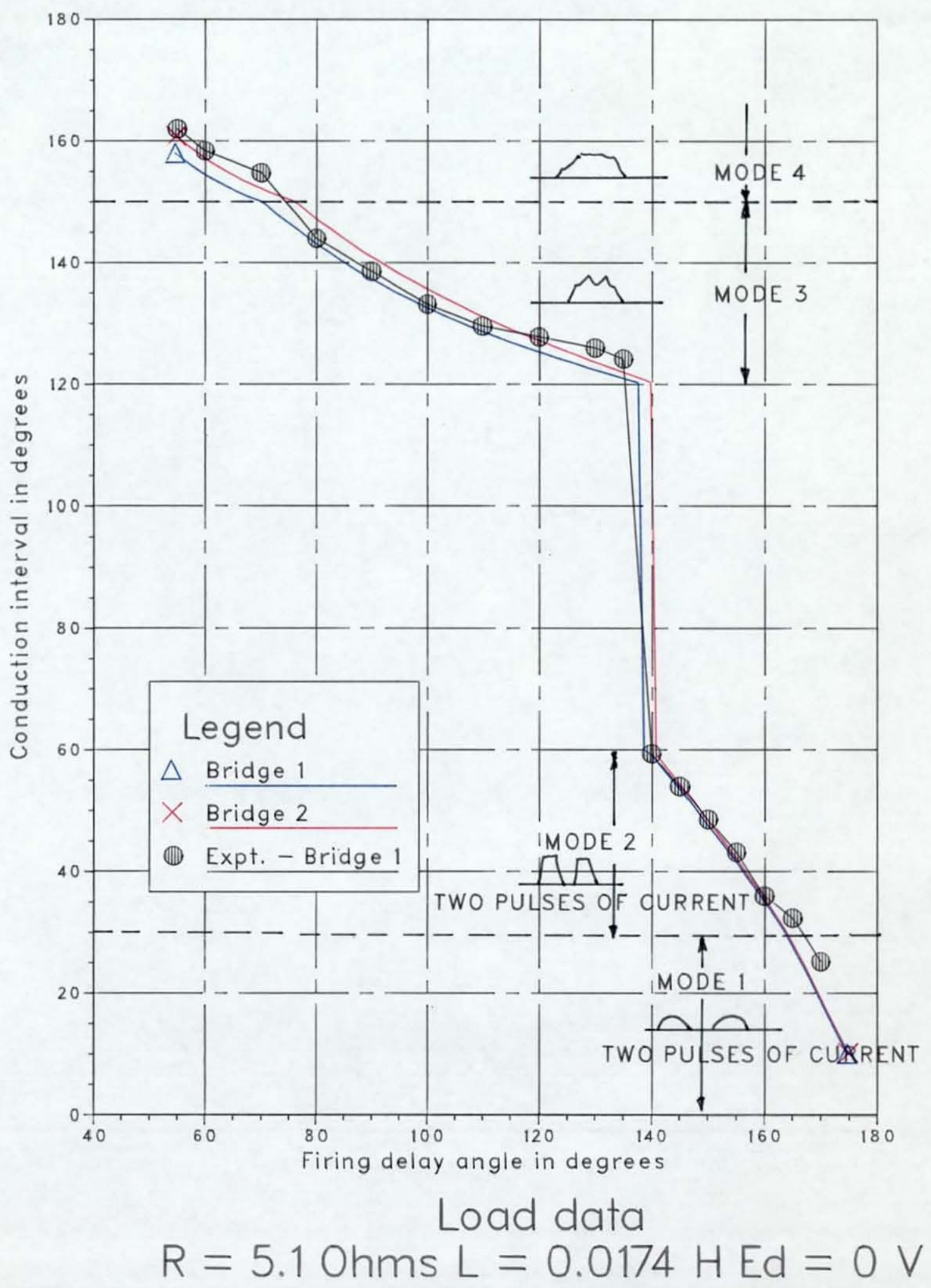


FIG. 6.23 INTERBRIDGE COMMUTATION CHARACTERISTK MODE 2

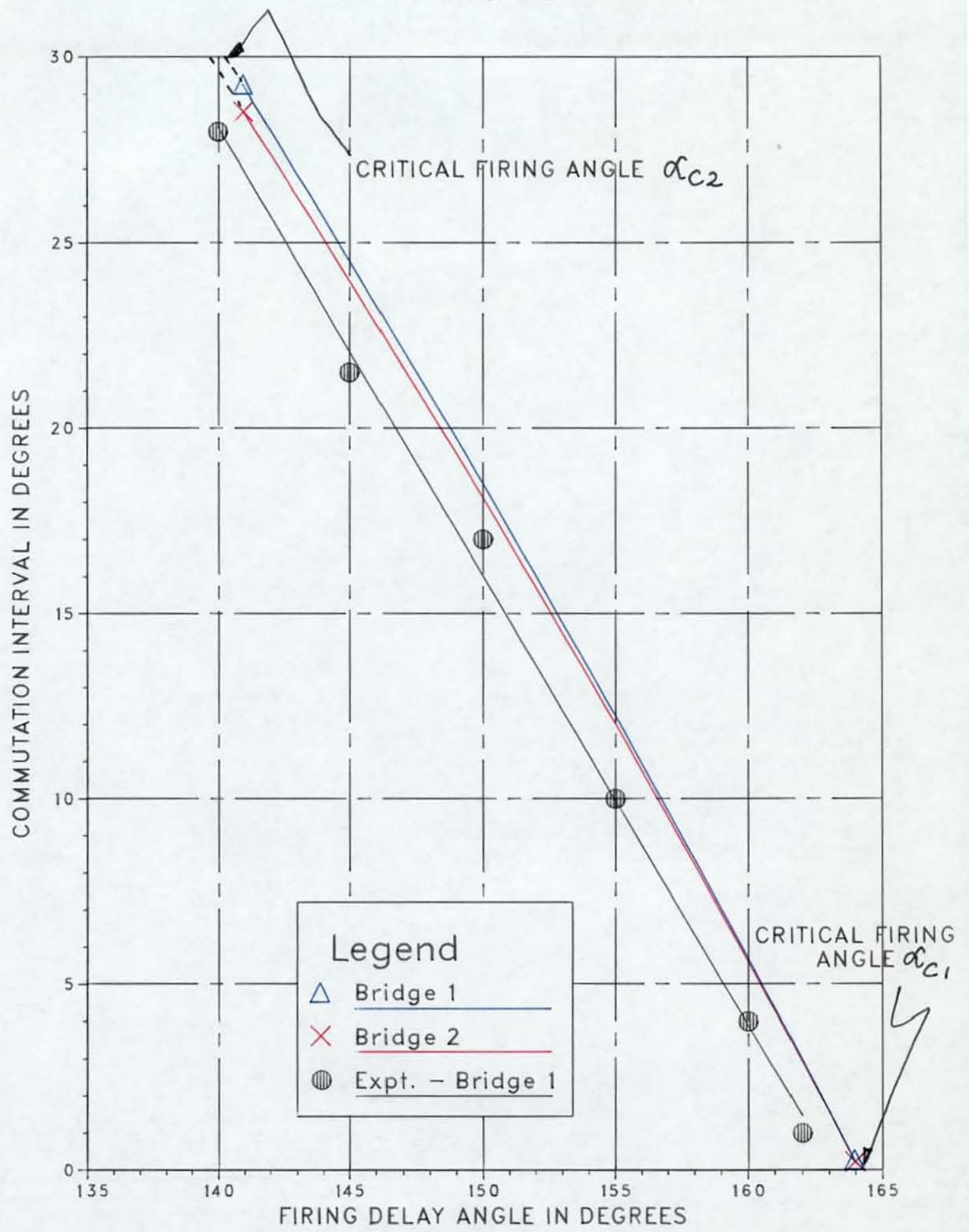

Load data

$\mathrm{R}=5.1 \mathrm{ohms} \quad \mathrm{L}=0.0174 \mathrm{H} \quad \mathrm{Ed}=0 \mathrm{~V}$ 
FIG. 6.24 INTERPHASE COMMUTATION CHARACTERISTIK MODE 3

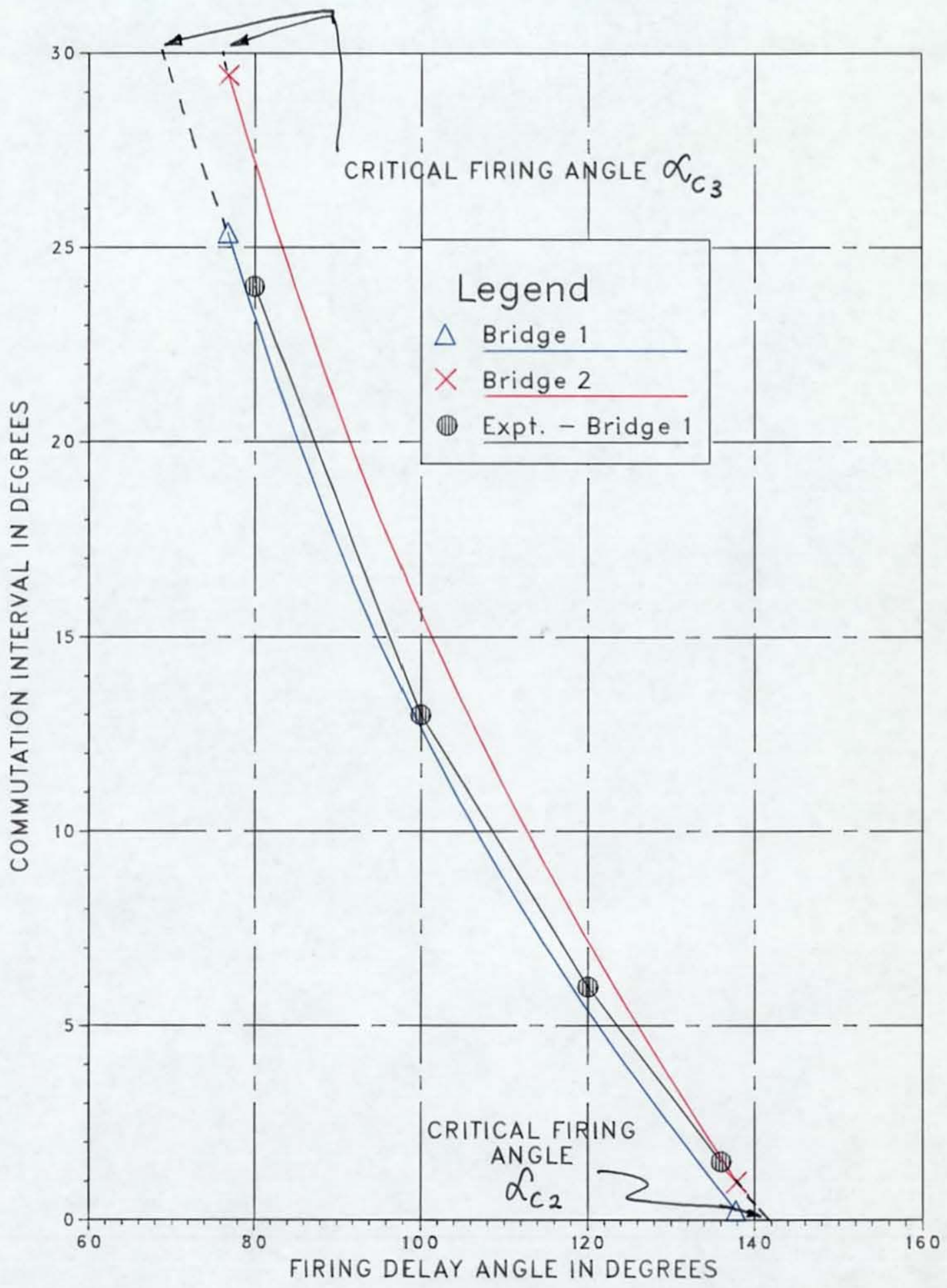

Load data

$R=5.1 \mathrm{Ohms} \mathrm{L}=0.0174 \mathrm{HEd}=0 \mathrm{~V}$ 
FIG. 6.25 OVERLAPPING INTERPHASE COMMUTATION CHARACTERISTIC:

MODE 4

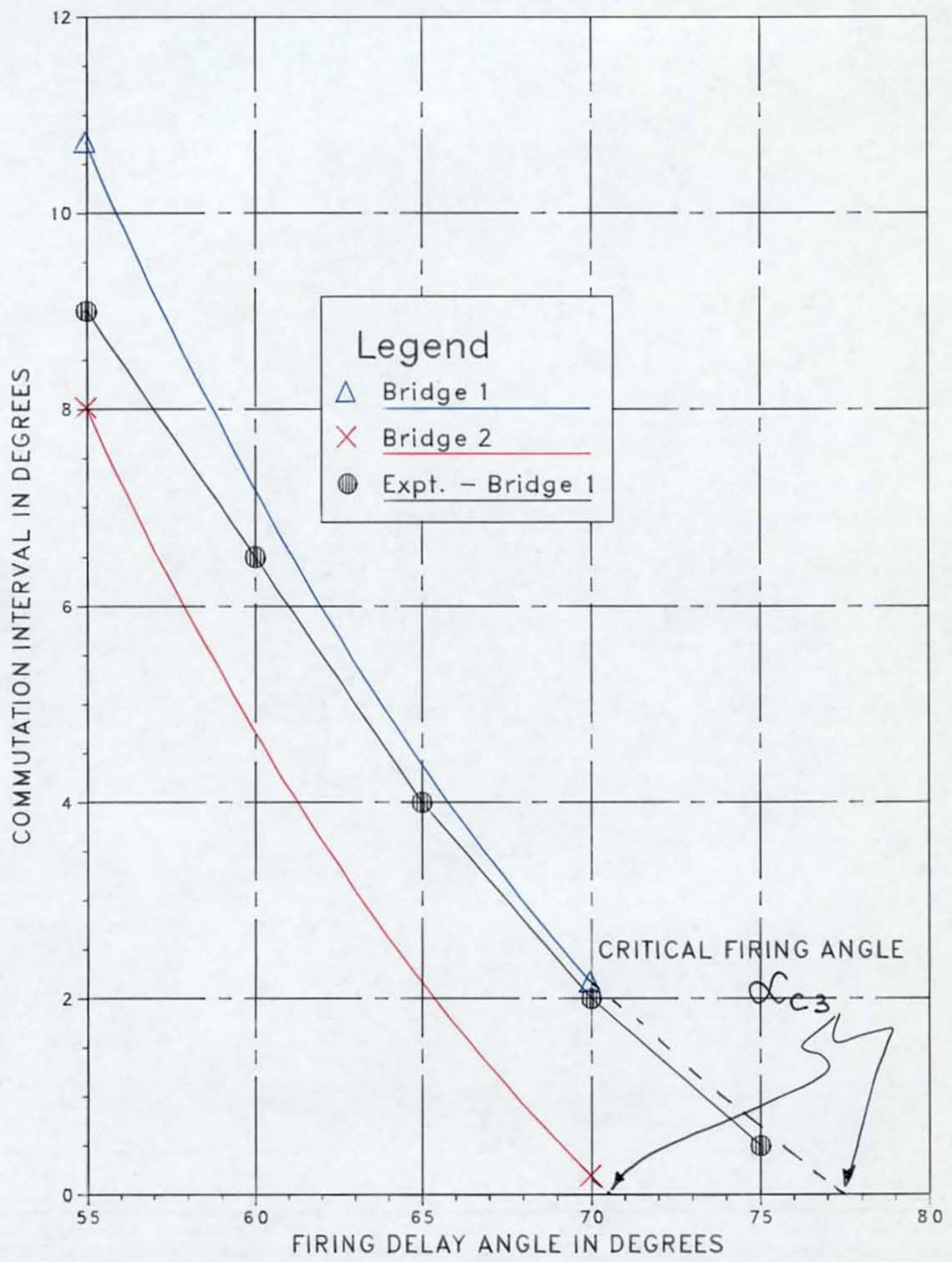

Load data

$\mathrm{R}=5.1 \mathrm{Ohms} \mathrm{L}=0.0174 \mathrm{HEd}=0 \mathrm{~V}$ 
FIG. 6.26 THE AC SUPPLY LINE CURRENT HARMONIC CHARACTERISTICS

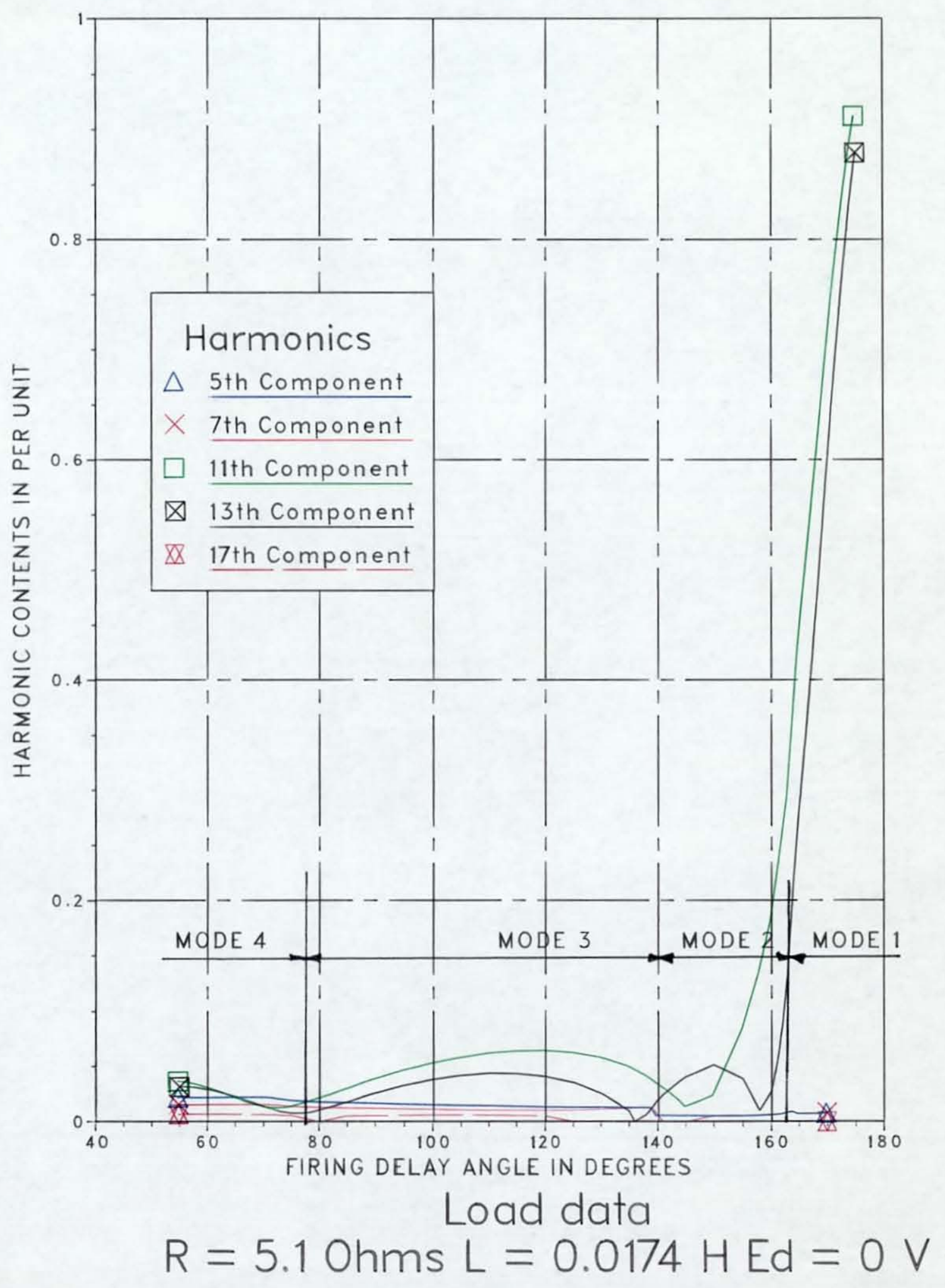


FIG. 6.27 THE AC SUPPLY LINE CURRENT CHARACTERISTIC HARMONICS

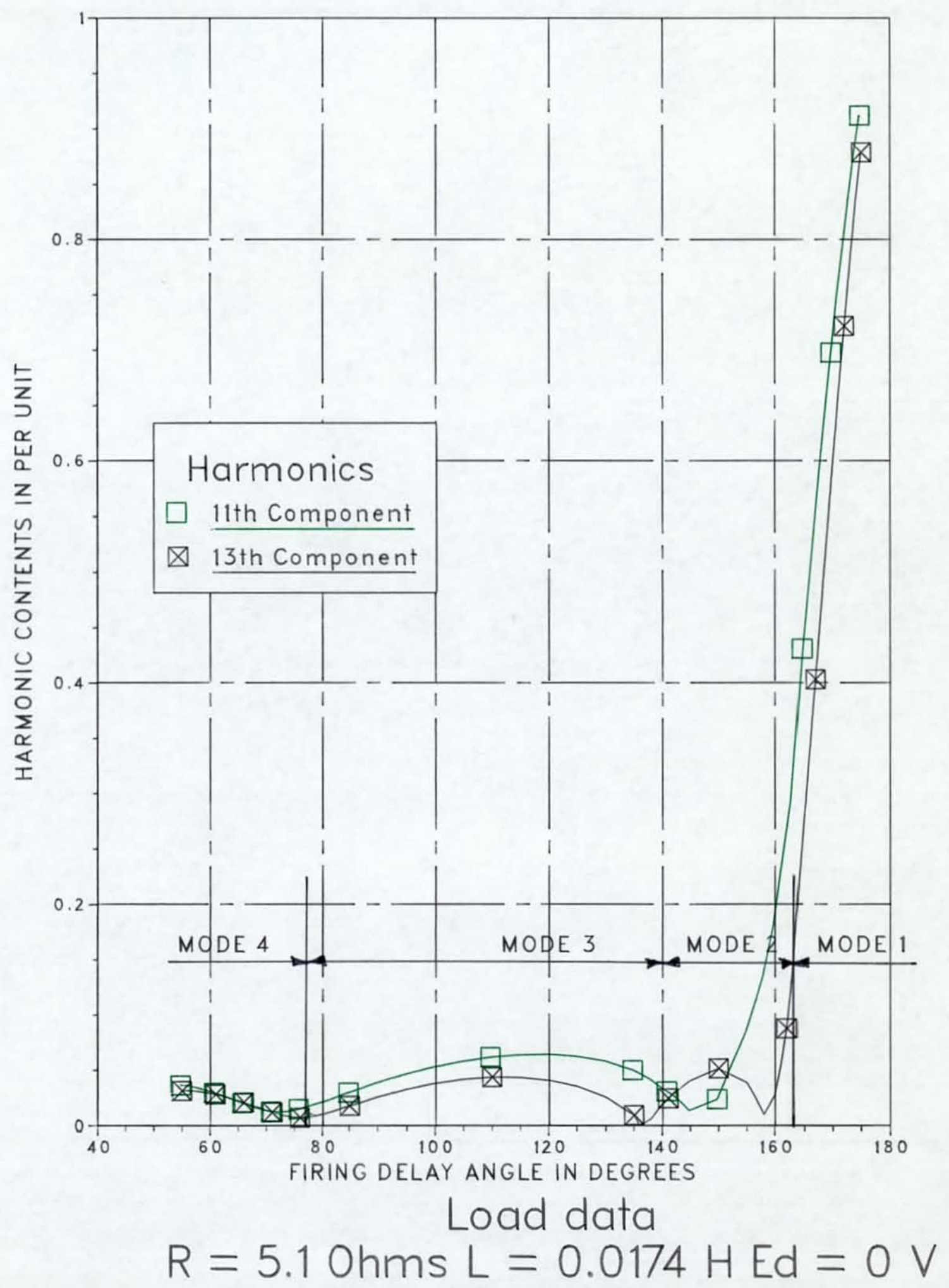


FIG. 6.26 THE AC SUPPLY LINE CURRENT UNCHARACTERISTIC HARMONICS

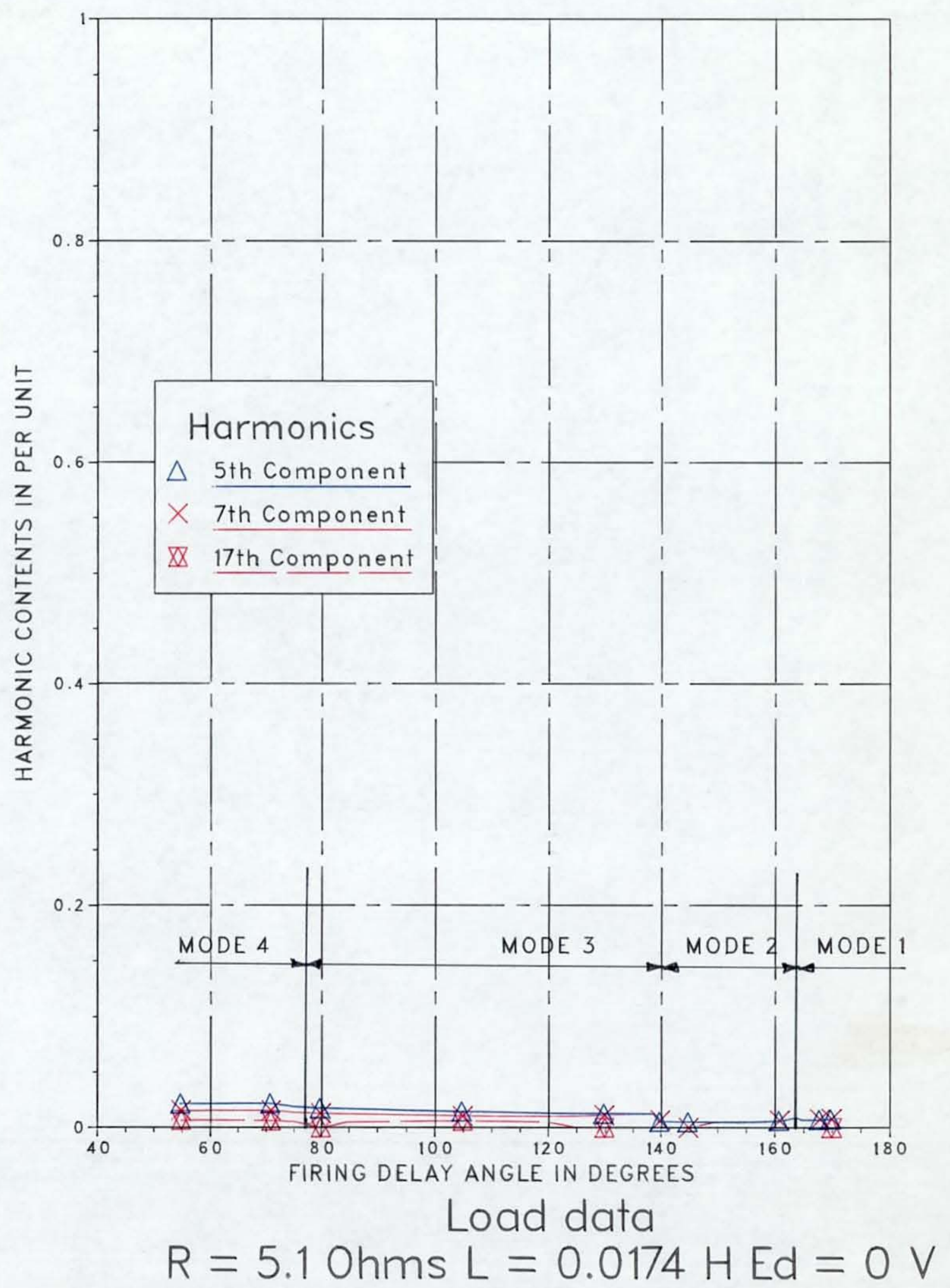


comparison with experiment, black dots are included to denote measured values.

The device conduction interval varies with the firing delay angle as shown in Fig. 6.21. It exhibits well the mode changes as the firing delay angle alters. The conduction interval is measured for a single pulse of bridge current which, for Modes 1 and 2, is below $60^{\circ}$. The jump from $60^{\circ}$ to $120^{\circ}$ results when the two $60^{\circ}$ current pulses merge. Mode 3 conduction lies between $120^{\circ}$ and $150^{\circ}$ and Mode 4 conduction between $150^{\circ}$ and $180^{\circ}$.

As shown in Chapter 2 the minimum possible operating firing delay angle measured from voltage zero is $75^{\circ}$ for an assumed sinusoidal supply voltage. Here on full load the minimum $\alpha$ reduces to $55^{\circ}$. This is brought about by the extreme distortion of the bridge supply voltages which provides positive anode-cathode bias for the thyristors over the additional range of $75^{\circ} \leqslant \alpha \leqslant 55^{\circ}$. Fig. 6.29 shows the distorted supply voltage at $\alpha=55^{\circ}$. The distortion arises from the normal switching of the devices and the voltage drops across the transformer impedance components.

The interbridge, interphase and overlapping of interphase commutation characteristics (Figs. 6.23, 6.24 and 6.25) are plotted for the range of $\alpha$ corresponding to the related mode of operation shown. The commutation angle (or overlapping as appropriate) varies from $0^{\circ}$ to $30^{\circ}$ in each case. Fig. 6.23 shows a linear relationship between the interbridge commutation and firing delay angles while that of Figs. 6.24 and 6.25 exhibit some small non-linearity for the interphase commutation characteristics. 


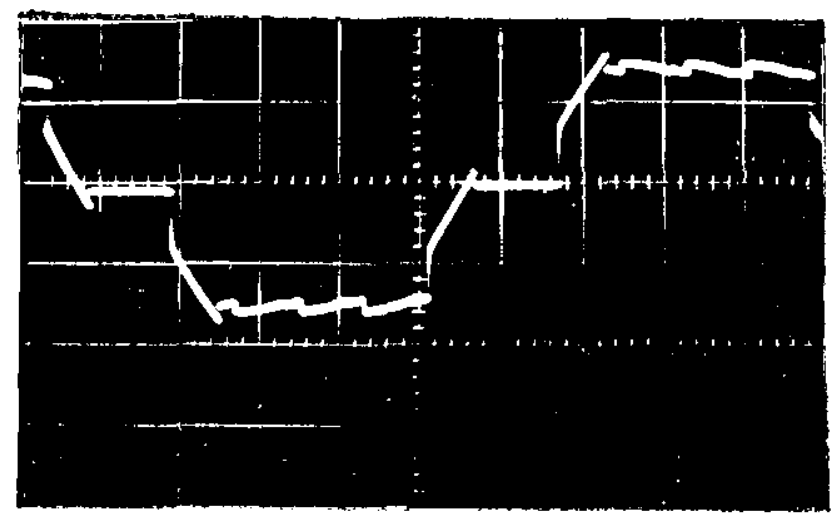

FIG. 6.29 THE DISTORTED BRIDGE SUPPLY VCITAGE AT FULI ICAD i.e $\boldsymbol{\alpha}=55^{\circ}$ 
The computed conduction and comutation angles show differences of value for the bridges, appreciably so for the interphase commutations within the bridges (Mode 3 ) and the overlapping of interphase commutations between the bridges (Mode 4). Despite balanced transformer impedances being used in the computer program, for complete equivalence the delta values should be three times the star values for the two secondary windings. Also, the mutual inductance of the primary and the delta secondary windings should be $\sqrt{3}$ times the mutual inductance of the primary and star secondary windings. In setting up the transformers it was found practicable to make these so for the inductances but not for the resistances. This accounts for the variation of characteristics between the bridges. A re-run of the computer program with the resistances so adjusted produces complete operating balance.

Differences between measured and computed values may be attributed to the reasons listed in the previous section. The harmonic contents of the total ac supply line current in Fig. 6.25 are plotted up to the 17th component. Two accompanying plots (Figs. 6.26 and 6.27) are also presented which differentiate these harmonics into the characteristic and uncharacteristic ones. For the ac supply line current waveform, the lowest characteristic harmonics are the 11th and 13th while the 5 th, 7 th and 17 th are uncharacteristic. For an ideal rectangular block waveform, the latter are zero (ref. 24 and 25). Both the 11th and 13th components exhibit a significant change as the operation moves from one mode to the next. Both fall to zero at firing delay angles which are within a few degrees of the critical values between the modes except between 
Modes 1 and 2 where bridge current conduction is discontinuous and they remain high, being on an increasing characteristic as the load current conduction interval becomes shorter. Apart from these very high levels, with continuous bridge current the peak level is about 68 of the fundamental. As might be expected, the uncharacteristic harmonics have extremely low values, even down to virtually zero conduction angle. They are present due to system and firing angle unbalance (refs. 24 and 25). 


\subsection{Summary of work done}

This thesis has described an investigation into the operation and performance of two bridge rectifiers connected in parallel, without an interbridge reactor and supplied from a three-winding transformer. In particular it has covered:

(a) the definition and description of the operating modes;

(b) the analysis of the system and its mathematical modelling, the model developed being sufficiently flexible to incorporate an interbridge reactor if required;

(c) the implementation of the model in a computer program;

(d) the development of an experimental laboratory arrangement to verify the predicted results from the model;

(e) computation of the transformer and ac supply current waveforms and their experimental verification;

(fl harmonic analysis of the current waveforms noted in (e) and experimental verification;

(g) computation of the operating characteristics of the system with experimental verification.

\subsection{Discussion}

The omission of the interbridge reactor for paralleled rectifier circuits provides advantages, especially from the economical point of view. This has particular attraction with large, high current rectifiers for electrochemical application. In such cases, it is usual to design the main transformer with the secondary windings having equal reactances which are high relative to the primary winding 
reactance to avoid high circulating components between the bridges. The reactances referred to here are those of the transformer equivalent circuit (Fig. 6.4), and normally known as the leakage reactances. A rigorous analysis of such a system has apparently not been undertaken before, the possible reasons being;

(a) the complexity;

(b) the lack of perception of the practical need.

This has now been achieved in this research.

The particular features of the analysis are:

(i) no assumptions such as infinite load inductance are made;

(ii) no separately derived equivalent circuit is used; the model is developed from first principles including all winding self and mutual inductances, and winding resistances;

(iii) the model is adaptable to parallel rectifjer systems where an IPT is included; it can be used when a three phase transformer is used or single three-winding transformers for each phase, as here;

(iv) thyristor conduction drop has been incorporated;

(v) the dc load considered includes all the typical elements of resistance, inductance and back emf.

The mathematical model has been shown to be accurate by the satisfactory correlation between the computed results and those obtained from the experimental laboratory system bearing in mind that the transformer impedance and voltage balance, and firing delay balance, is not perfect in the latter. Perfect balance of firing delay and transformer impedances between phases is assumed in the model. Far better balance can be achieved in industrial transformers, 
purpose built for the application. The model has been developed using tensor techniques.

Since the model requires transformer parameters including total and mutual inductances rather than the conventional percentage or per unft winding leakage inductances, the data requirements are somewhat unconventional in relation to industrial practice.

The need for such a detailed and fundamental approach arises due to the use of a three-winding supply transformer, the conventional equivalent circuit per phase for which has been found to be unsatisfactory for the purpose of this analysis since it does not take into account all interphase mutual coupling. Additionally it was found difficult to use in conjunction with the rectifier switching action.

The program automatically determines the operating mode and provides a print out of the ac side current waveforms, harmonic components and the conduction, interbridge, interphase and overlapping interphase commutation angles. Additionally, the mean values of the dc output voltage, individual bridge and total output currents are given. Thus a full range of operating characteristics are available. The need for a more accurate technique than has been used hitherto for the calculation of the harmonic current components in the rectifier transformer windings when an interbridge reactor is not used, has provided the incentive for this work. It is hoped that the research has made a valuable contribution towards fulfilling this.

\subsection{Suggestions for further work}

(i) The study of the 12-pulse parallel bridge system can be extended to include the effects of voltage and transformer impedance unbalance; 
and thyristor firing delay angle asymmetry. This, however, will be required only in situations where such problems are particularly severe and would involve considerable additional complication in the analysis and model.

(ii) Due to the versatility of the tensor technique, ac side filter circuits could be included in the model of the system if required. (iii) The operating characteristics may be developed into a more general normalised form, rather than be related to specific impedance values.

(iv) The developed mathematical model could be modified into a package form for use with a microcomputer. This would make it more convenient for the rectifier system designer. 


\section{REFERENCES}

1. Electricity Council Engineering Recommendation G5/3:

'Limits for Harmonics in the U.K. Supply System'.

2. SOOD, P.K.: 'Prediction of AC Line Current Harmonics for Non-Classical Thyristor Controlled Loads'. (M.Sc Thesis, 1977).

3. SEYMOUR, J.: 'Semiconductor Devices in Power Engineering',

A Symposium held at Woolwich Polytechnic, (Pitman, 1968)

4. LANGLOIS-BERTHELOT, R. : 'Transformers and Generators for Power Systems', (London Macdonald, 1960).

5. HALL J.K., KETTLEBOROUGH J.G. and RAZAK M.J. A.: 'Current Harmonics from Parallel Bridge Rectifiers', 17th UPEC, UMIST, April 1982.

6. RAMAMOORTY, M. : 'An Introduction to Thyristors and their Applications'. (Macmillan Press Limited, 1978)

7. SMITH, M.J. : 'Modelling and Analysis of Non-ideal Polyphase Diode Converters', Simulation (USA), 1971, 17, pp 237-243.

8. HAY, J.I. and HINGORANI, N.G. : 'Dynamic Simulation of Multiconverter hvdc Systems by Digital Computer', Pt.1 - Mathematical Model, IEEE Trans., 1970, PAS-89, pp.218-222 and Pt.2 - Computer Program, ibid., pp.222-228.

9. HTSUI, J.S.C. and SHEPHERD, W. : 'Method of Digital Computation of Thyristor Switching Circuits', Proc. IEE, 1971, 118, (8), pp. 993-998. 
10. HINGORANI, N.G., HAY, J.L., and CROSBIE, R.E. :

'Dynamic Simulation of hvdc Transmission Systems on Digital Computers', ibid, 1966, 113, (5), pp. 793-802.

11. O'REGAN, P.G., and DILLON, C.T.G.: 'Digital Simulation of Transients in hvdc Convertors with Harmonic Filters', ibid, 1970, 117, (2), pp. 421-430.

12. WILLIAMS, S, and SMITH, I.R. : 'Fast Digital Computation of 3-phase Thyristor Bridge Circuits', ibid, Vol.120, No.7, 1973, pp. 791-795.

13. WILLIAMS, S, and SMITH, I.R.: 'SCR Bridge Converter Computation using Tensor Methods', IEEE Trans., 1976, C -25 , pp.1 - 6 .

14. KETTLEBOROUGH, J.G., SMITH, I.R., and FANTHOME, B.A.: 'Simulation of a Transformer/Rectifier Unit for Aircraft Power-Supply Systems', IEE Proc., Vol.129, Pt.B, No.6, 1982, pp. 323-329.

15. KRON, G. : 'Tensor for Circuits', (Dover, 1959)

16. KRON, G. : 'Tensor Analysis of Networks, (Wiley, 1939).

17. HAPP, H.H. : 'Diakoptics and Networks', (Academic Press, 1971).

18. New Users' Introduction to Multics-Part 1 ;

(Honeywell, November 1979).

19. WILLIAMS, S. : 'Tensor Techniques for the simulation of Alternator System Containing Thyristors', Ph.D. Thesis, University of Birmingham, 1974.

20. KEMP, P. : 'Alternating Curxent Waveforms - Theory and Practice', Vol.I, (Chapman and Hall Ltd., 1952), A series of monographs on electrical engineering. 
21. FICH, S. and POTTER, J.L. : 'Theory of ac Circuits', (London, Macmillan and Co. Ltd., 1959).

22. VAN VALKENBURG, M.E. : 'Network Analysis' (Prentice-Hall, Inc. Ma ruzen Co.Itd., 1964).

23. FRANKLIN, A.C. : 'The J\&P Transformer Book', (Butterworths, 11th Ed., 1983)

24. MATHUR, R.M. : 'A study of non-characteristic harmonics generated by thyristor phase controlled reactors', IEE International Conf., Pub. No.25, pp. 117-120.

25. KIMBARK, E.W. : 'Direct Current Transmission', Vol.I, (Wiley-Interscience, New York, London, 1971). 


\section{APPENDIX I}

\section{MEASUREMENT AND CALCULATION OF TRANSFORMER PARAMETERS}

\section{I.1 Measurement}

The open-circuit test was employed to determine the transformer parametexs. This was carried out on the three phase basis. Fig. I.l below shows the circuit measurement and Table I.l provides the readings of the meters in the circuit. The measurement was repeated with the three-phase supply to each transformer windings.
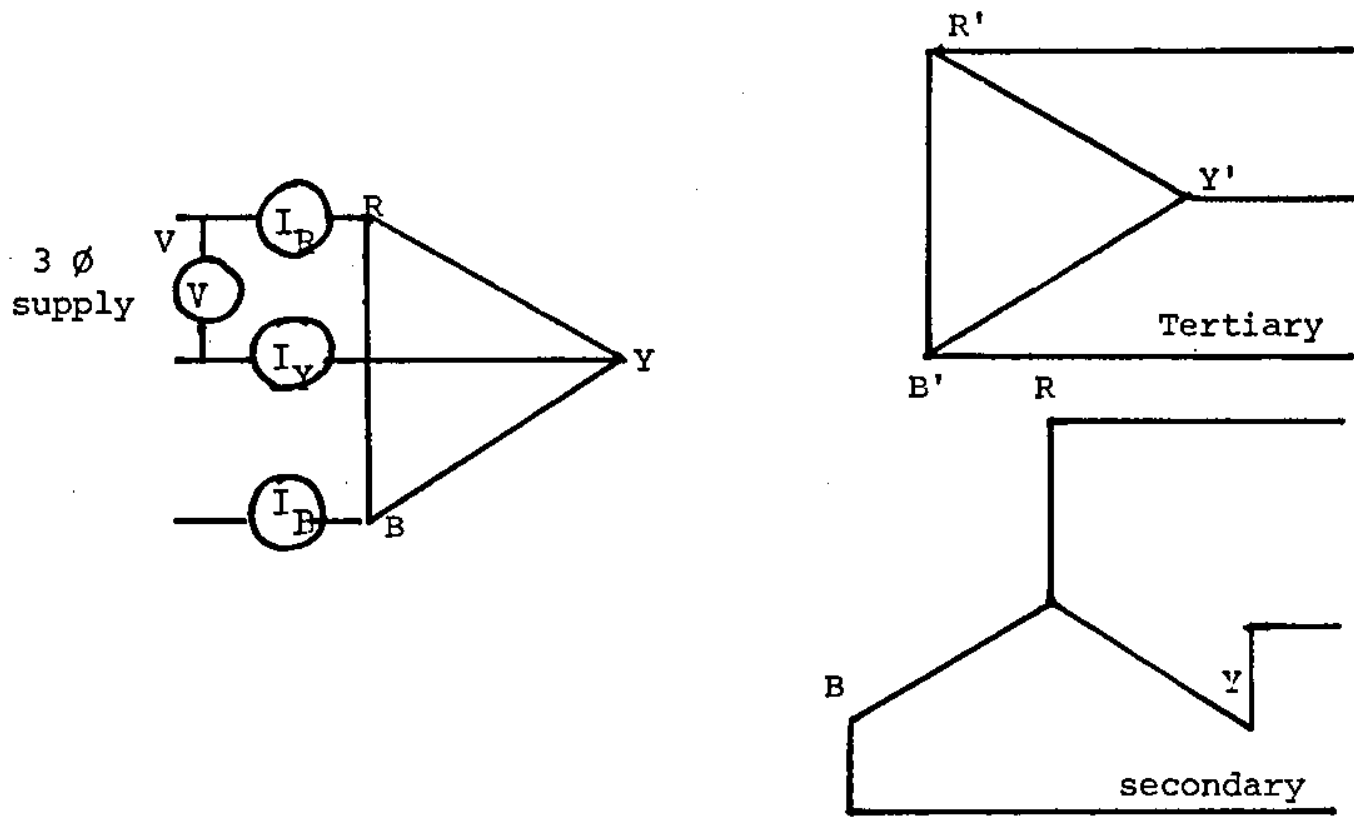

Fig. I.1 Open-Circuit Test for the 3-winding Transformer

The dc resistance of the windings was measured using a digital resistance meter and the readings of each winding resistance is produced in Table I.2. 


\begin{tabular}{|c|c|c|c|c|c|c|c|c|c|c|c|c|}
\hline & \multicolumn{3}{|c|}{$\begin{array}{l}\text { line currents of } \\
\text { the windings as } \\
\text { defined (A) }\end{array}$} & \multicolumn{3}{|c|}{$\begin{array}{l}\text { primary } \\
\text { phase voltage } \\
\text { (V) }\end{array}$} & \multicolumn{3}{|c|}{$\begin{array}{l}\text { secondary } \\
\text { phase voltage } \\
\text { (v) }\end{array}$} & \multicolumn{3}{|c|}{$\begin{array}{l}\text { delta tertiary } \\
\text { phase voltage } \\
\text { (V) }\end{array}$} \\
\hline & $I_{R}$ & $I_{Y}$ & $I_{B}$ & $\mathrm{R}$ & $Y$ & B & $\mathrm{R}$ & $\mathbf{Y}$ & B & $R$ & $Y$ & B \\
\hline a & 0.81 & 0.76 & 0.78 & 236.3 & 235.2 & 235.8 & 125.5 & 125.63 & 125.96 & 125.55 & 125.6 & 126.18 \\
\hline b & 1.37 & 1.32 & 1.39 & 228.8 & 228.6 & 227.5 & 126.0 & 125.65 & 125.79 & 119.2 & 120.27 & 119.72 \\
\hline c & 1.42 & 1.33 & 1.36 & 228.3 & 226.9 & 227.6 & 119.22 & 119.82 & 119.66 & 125.81 & 125.58 & 125.84 \\
\hline
\end{tabular}

TABLE I.1: Open circuit test readings of Fig. I.1

Note: a. 3-phase supply to the primary winding

b. 3-phase supply to the star secondary winding

c. 3-phase supply to the delta tertiary winding 


\begin{tabular}{|c|c|c|c|}
\hline Resistance of & $\mathrm{R}-\mathrm{Y}$ & $\mathrm{R}-\mathrm{B}$ & $\mathrm{Y}-\mathrm{B}$ \\
\hline The primary windings $(\Omega)$ & 0.353 & 0.471 & 0.462 \\
\hline The star secondary windings $(\Omega)$ & 0.578 & 0.488 & 0.609 \\
\hline The delta tertiary windings $(\Omega)$ & 0.144 & 0.204 & 0.178 \\
\hline
\end{tabular}

Table I.2 DC resistance of the transformer windings

\section{I.2 Calculation}

The following relationships are used to obtain the transformer parameters. These represent the windings 1,4 and 7 of Fig. 3.1.

$$
\begin{aligned}
& v_{1}=r_{11} i_{1}+L_{11} \frac{d i_{1}}{d t}+L_{14} \frac{d i_{4}}{d t}+L_{17} \frac{d i_{7}}{d t} \\
& v_{4}=L_{41} \frac{d i_{1}}{d t}+r_{44} i_{4}+L_{44} \frac{d i_{4}}{d t}+L_{47} \frac{d i_{7}}{d t} \\
& v_{7}=I_{71} \frac{d i_{1}}{d t}+L_{74} \frac{d i_{4}}{d t}+r_{77} i_{7}+L_{77} \frac{d i_{7}}{d t}
\end{aligned}
$$

These relationships become, when $i_{4}=i_{7}=0$,

$$
\begin{aligned}
& v_{1}=r_{11} i_{1}+I_{11} \frac{d i_{1}}{d t} \\
& v_{4}=I_{41} \frac{d i_{1}}{d t} \\
& v_{7}=L_{71} \frac{d i_{1}}{d t}
\end{aligned}
$$

hence for a steady state system,

$$
\begin{aligned}
& v_{1}=r_{11} I_{1}+j \omega L_{11} I_{1} \\
& v_{4}=j \omega L_{41} I_{1} \\
& v_{7}=j \omega L_{71} I_{1}
\end{aligned}
$$


similarly, when $i_{1}=i_{7}=0$, give,

$$
\begin{aligned}
& v_{1}=j \omega \mathrm{I}_{14} I_{4} \\
& v_{4}=r_{44} I_{4}+j \omega L_{44} I_{4} \\
& v_{7}=j \omega L_{74} I_{4}
\end{aligned}
$$

and, when $i_{1}=i_{4}=0$, give

$$
\begin{aligned}
& v_{1}=j \omega L_{17} I_{7} \\
& v_{4}=j \omega I_{47} I_{7} \\
& v_{7}=I_{77} I_{7}+j \omega L_{77} I_{7}
\end{aligned}
$$

From Table I.l and Table I.2 taking the average values of the currents and voltages and adding 108 to the average dc resistance of the windings (i.e. to obtain the equivalent ac winding resistance) respectively, the self and mutual inductances are calculated as given in Table 6.3 . 
APPENDIX II

\section{CIRCUIT DETAILS FOR THE LABORATORY SYSTEM}

Fig. II.1 represents the control circuit for the parallel bridge system used in the research. The two phase shifting transformers (Magslips) are clearly shown indicating their respective functions. The output of these transformers are also shown connected to a $3 / 6$ phase transformer for connection to the pulse circuits. Fig. II.2 shows the pulse circuit for one of the thyristors. 
FTG. II.1 .RECTIPIER FIRTNG CONTROL SCHPMATIC AND THE POLSE CIRCUIT

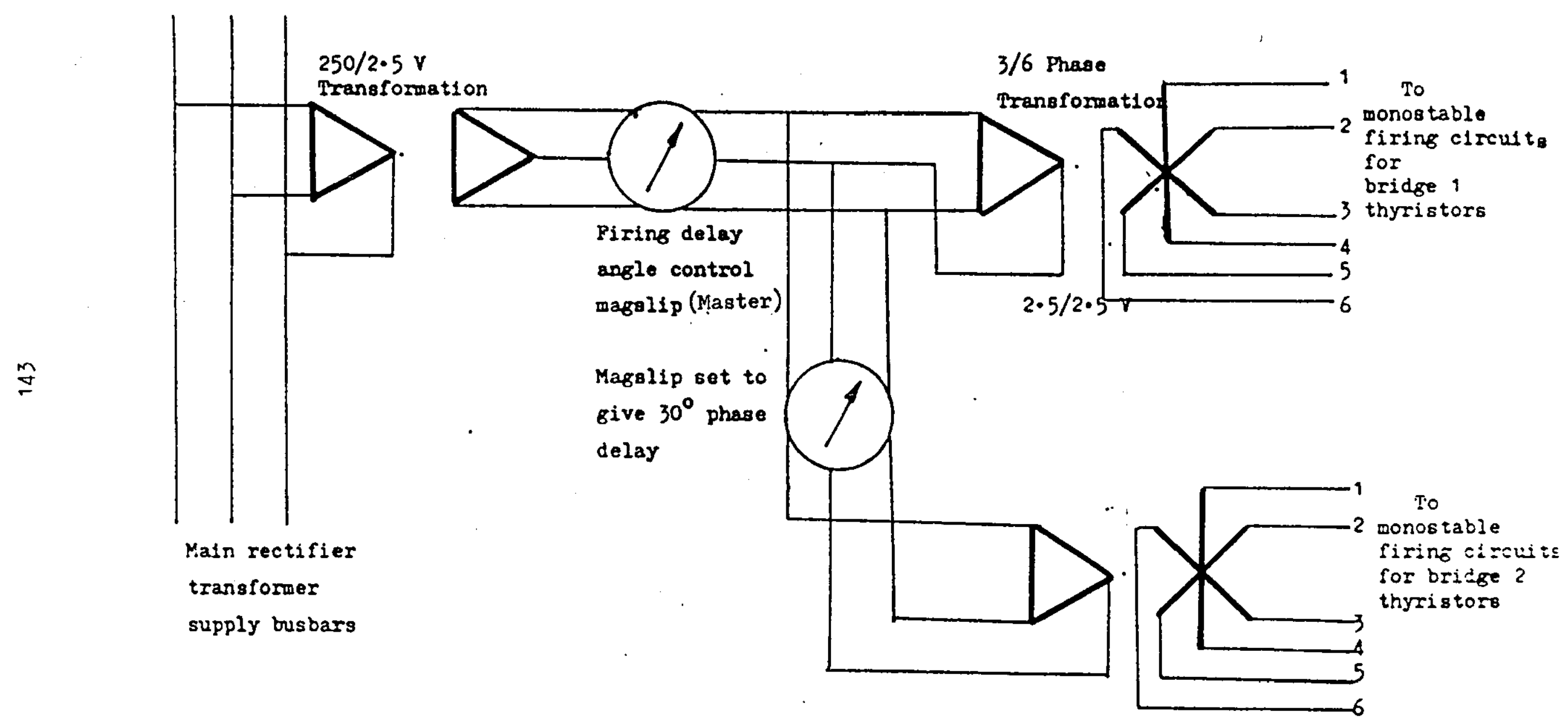


FIG. II.2 TULSE CIRCOIT FOR ONE THYRISTOR

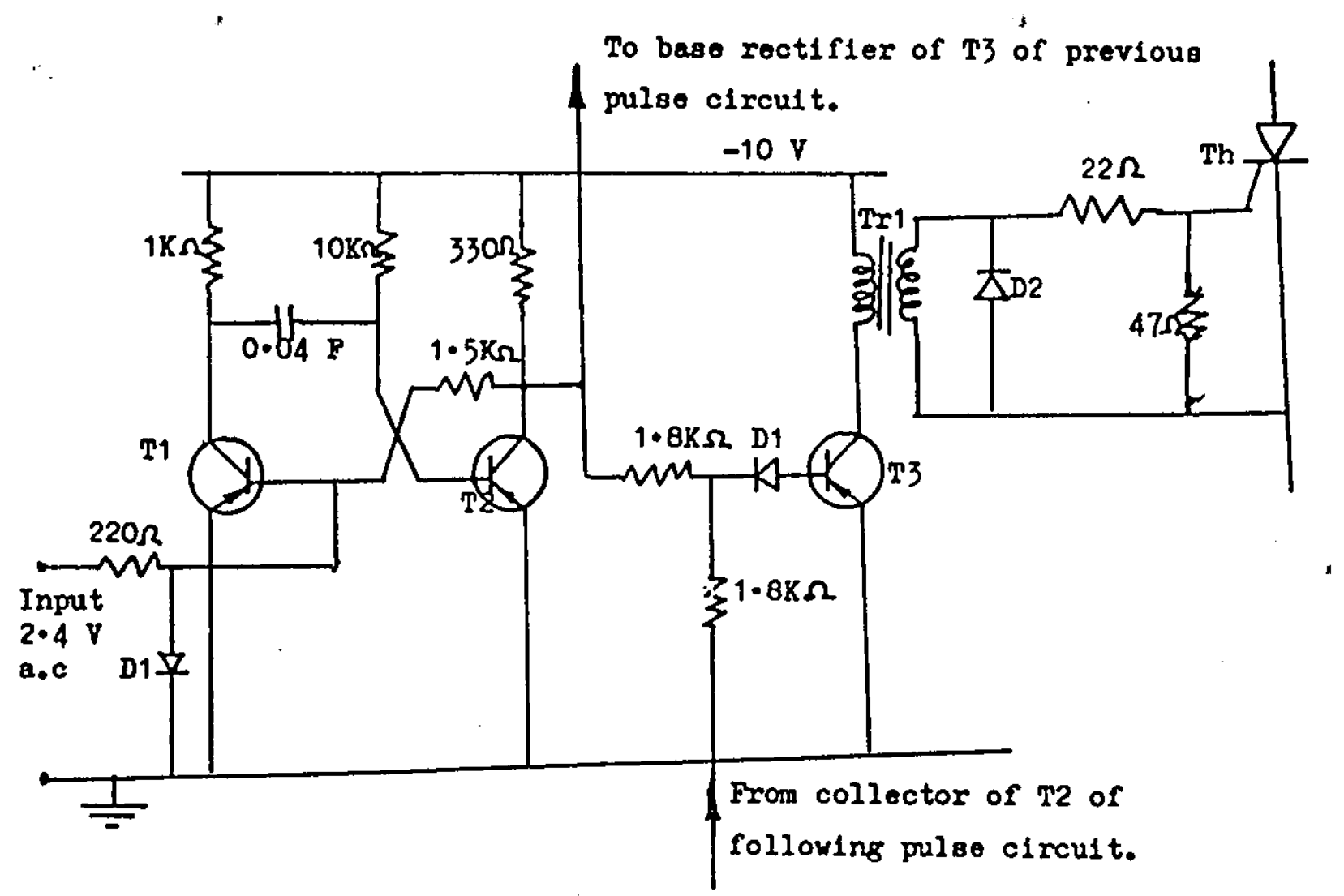

T1 and T2 - Transigtor, Mulard, type GFiT 113 or GET 114

T3 - Transistor, Mulard, type GET 115 or GET 114

Tr1 - Output transformer, Telcon - type 3 A core wound with 1000 turn primary and secondary of $\cdot 004^{\prime \prime}$ strip wound with 600 turns.

D1

- Silicon diode, Mulard, type GEX 23

D2

- 'Texas' diode 


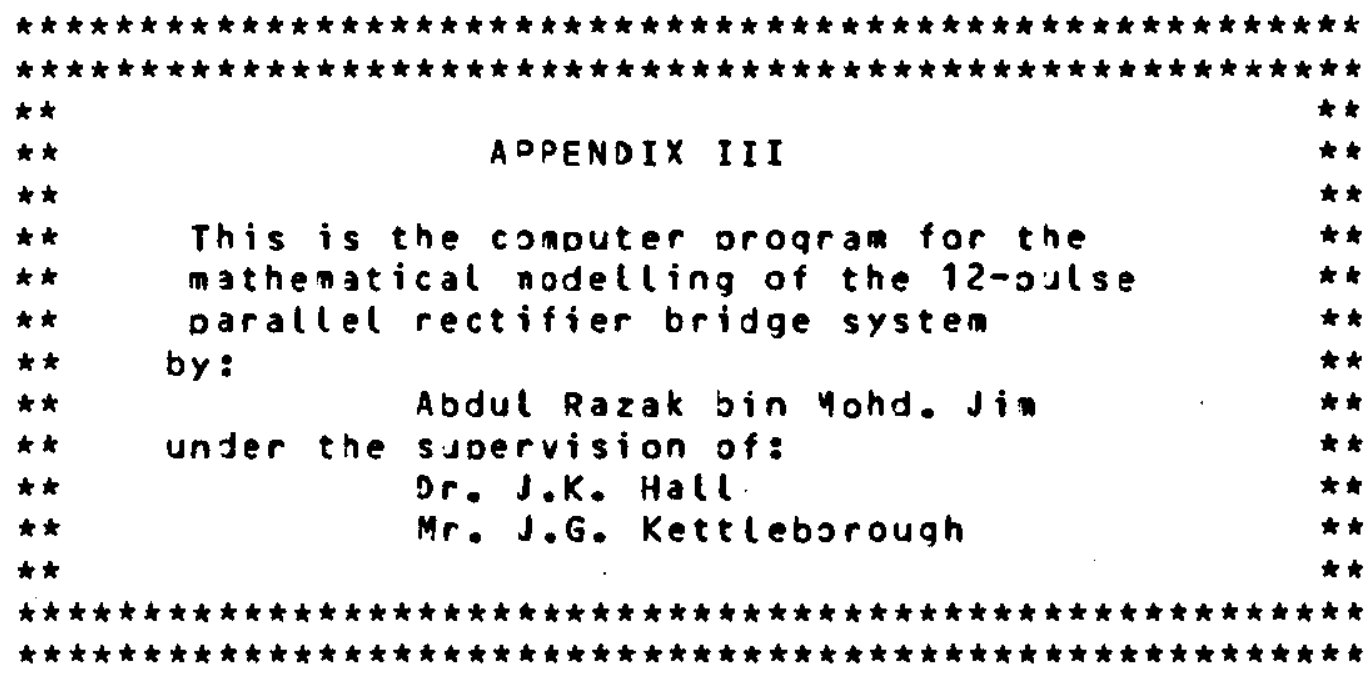

THE MAIN PROGRAM

inolicit real $* 8(a-h, a-w, z)$

Jimension ano(12),an(12), ab(24),vo(24), icl(12),

1vd(12), vdo(12) , rb (24.24), itria(13)

dimension cbran $(1.2,12), v(24)$

real*3 intvoxb(24.24)

real y1(500),y $2(500) \cdot y 3(500) \cdot y 4(500) \cdot y 5(500) \cdot y 6(500) \cdot x(500)$

reat $y 7(500) \cdot y 8(500)$

common/blk438/ikeij

common/blk2/cbran

common/blk3/ xoerb

common/blk156/dildt

common/blk521/vx

common/blk11/ab

common/blk12/vsevdo

common/blk892/axear

common /olk200/icl

common/blk201/vl

common/blk33/itria

commor/blk16/delt

common/blk24/tn

common/blk2s/eb,ethy

ooen( unit $=10$, file $={ }^{\circ} d a t a-16^{\circ}$, form $={ }^{\circ}$ fornatt $\left.=d^{\circ}\right)$

$i j=1$

$i k=1$

de $t t=.1 d-03$

set 2 ob natrix by sujroutine const and obtain the required inout data:

nstud- number of studies

nsub -number of subject

nol when equal 1 the waveforms are olotted when equal 0 no plot

tim - time in seconds

the firing delay anale is obtained from subroutine params

call consts

read (10,*) nstudenol

do 103 icnt $1=1$, nstud

call jarans 
read (10,*) nsub

inum $=1$

$t=1.0 x-09$

ts too $=t+d e t t$

c

c

234 continue

The Darallel bridge system is initiated by subroutine start

call start (an,ano,icl,mom2,t)

do 105 icnt $2=1$ onsub

read $(10, t)$ tim

num $=t i m / f e l t$

call load $(m)$

$c \operatorname{cose}(u n i t=10)$

call jostediterodeltom)

call label 2

to 107 icnt $3=1$, nus

The integration beains here

also the trigger oulses of the thyristor are set

checking of the oresense of a trigger oulse or mesh

current just joing nagative oy suroutine intero

calt wxrk4 (tetstopeaneano,meiter)

call triger(itrigetstoo)

iv $=0$

call interp(anคano,iel,tbodelt,menz,n,iv)

if $(n .29 .1)$ goto 202

int $v=d e t t-t b$

it $r a p=1$

$t s=t$

$t i=t+t b$

if (tn. (e.tb) goto 204

$t n=t n-t b$

$i v=1$

call nxrk4(tsotioan,ano,n2,iter)

call initcn(icl,ancano,mom2)

call orani(anemz)

call oranve(vbomz)

call enduct ( $v$ oovd)

do $104 \quad i=1.12$

$104 v d 0(i)=v d(i)$

call nxrk4(ti,tstoopanoano,meiter)

call intero(aneanjeicletbointvemenz,neiv)

if (n.:a.1)goto 202

int $v=i n t v-t b$

itrao $=i t r a p+1$

$t n=0.3 d+00$

$i v=0$

$t s=t i$

$t i=t i+t b$

if (itrao.te.5)90 to 204

stop

202

catl orint(tstopoabeitrig) 


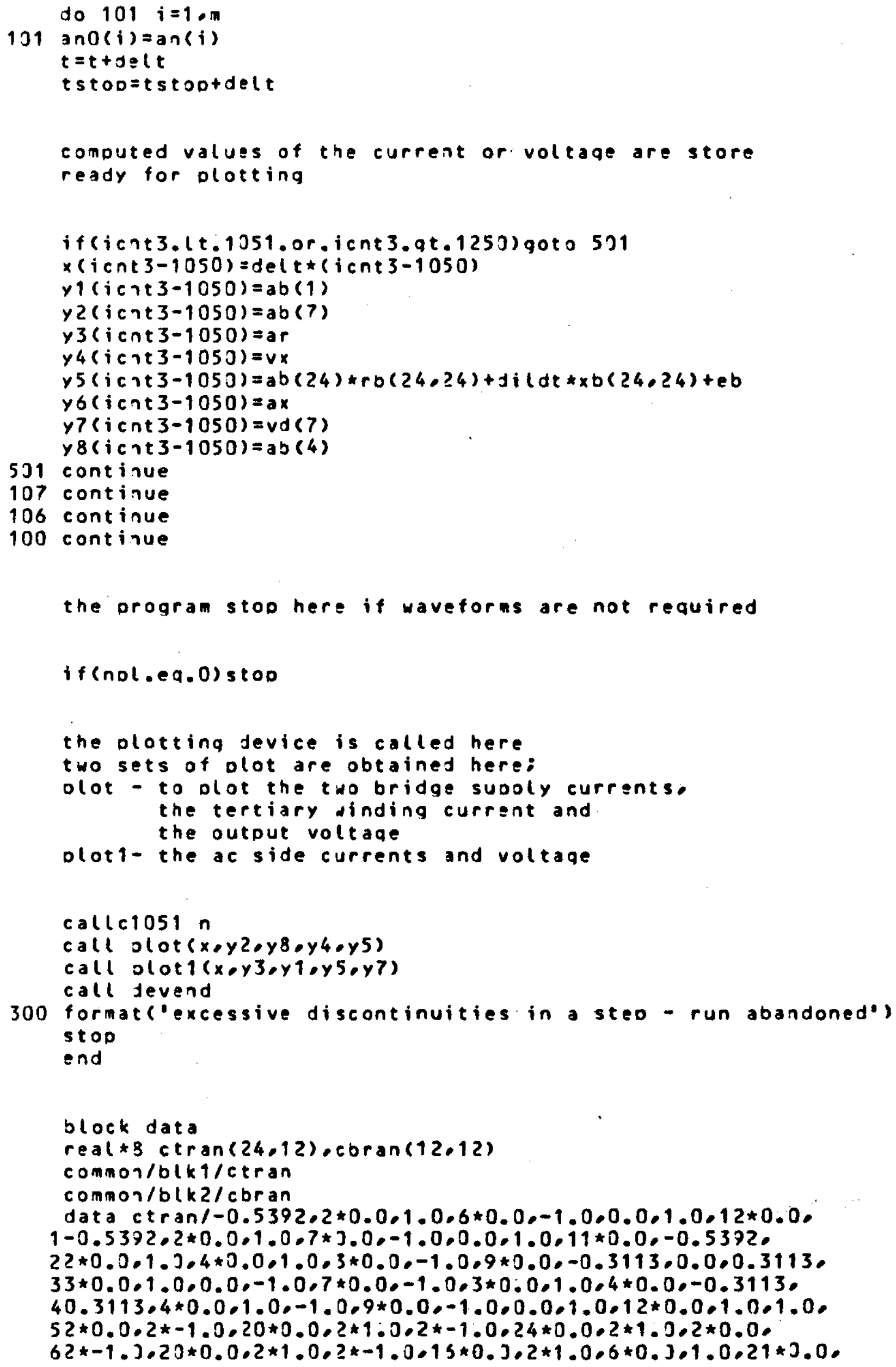


$72 * 1.0 .0 .0 .1 .0 .24 * 0.0 .1 .01$

data cbran $/ 1.0 .4 * 0.0 .1 .0 .3 * 0.0 .1 .0,0.0 .1 .0 .2 * 0.0 .-1.0 .2 * 0.0$,

$11.0 .3 * 0.0 .1 .0 .0 .0 .1 .0 .0 .0 .1 .0 .4 * 3.0 .1 .0 .2 * 0.0 .1 .0 .0 .0 .1 .0 .-1.0$.

$25 * 0.0 \cdot 1.3 .2 * 0.0 \cdot 1.0 .3 .0 \cdot 1.0 .2 * 0.3 \cdot 1.0 .6 * 0.0 \cdot 1 \cdot 0 \cdot 0.0 \cdot 1.0 .0 .0$.

$3-1.0 .7 * 0.0,1.0,0.0 \cdot 1.0,3 * 0.0,1.0,-1.0,2 * 0.0,1.0,2 * 0.0,2 * 1.0$.

$43 * 0.0 .1 .0 .3 * 0.0 .1 .0 .2 * 0.0 .2 * 1.0 .4 * 0.0 .1 .0 .3 * 0.0,1.0 .0 .0 .2 * 1.0$.

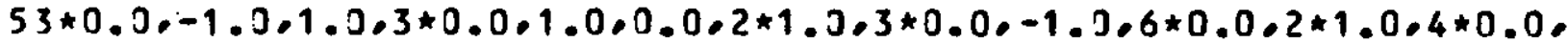
$6-1.0 .5 * 0.0 .2 * 1.01$

end

c

subrostine consts

inolicit real $\star 8(a-h, 0-2)$

dimension $x b: 24,24), r b(24,24), r(5), x(16)$

common/blk3/xborb

read $(10, t)(r(i), i=1,3)$

read $(10, *)(x(i), i=1,16)$

to $203 i=1,6$

c

write(5.500) ier (i)

600 format $\left(10 \times 0^{\circ} p^{\circ}, i 1 \mu^{\circ}=\right.$, 12.5$)$

200 continue

do $201 i=1.16$

$c$

write $(5.601)$ i.x(i)

601 format $\left(10 x 0^{\circ} x^{\circ}, i 20^{\circ}=\bullet .12 .5\right)$

201 continue

to $103 i=1.24$

do $103 j=1.24$

$r b(j, i)=0.0$

$100 \times b(j-i)=0.0$

do $11 \quad i=1,3$

to $10 j=1,3$

10

$x b(i, j)=x(7)$

$x b(i, i)=x(1)$

$r b(i, i)=r(1)$

12

do $12 j=4.6$

$\times b(j, j)=x(8)$

$\mathbf{i} 1=\mathbf{i}+3$

$x D(i, i 1)=x(2)$

do $13 j=7.9$

$13 \times 0(i, j)=x(9)$

$i 1=i+5$

$x b(i-i 1)=x(3)$

11

continue

c

c

15

do $14 i=4,6$

do $15 j=1.3$

$x b(i, j)=x(s)$

$i 1=i-3$

$x b(i, i 1)=x(2)$

15

do $16 j=4,6$

$\times b(j \cdot j)=x(10)$

$x b(i, i)=x(4)$

$r b(i, i)=r(2)$

do $17 j=7.9$

$17 \times b(j \cdot j)=x(11)$

$i 1=i+3$

$x b(i-i 1)=x(6)$

14 continue

c 
c

do $19 j=1.3$

$19 \quad \times b(i, j)=x(9)$

$i 1=i-5$

$x b(i, i 1)=x(3)$

do $20 j=4.6$

$20 \times b(i, j)=x(11)$

$i 1=i-3$

$x b(i, i 1)=x(6)$

do $21 j=7.9$

$21 \quad x b(i, j)=x(12)$

$x b(i, i)=x(5)$

$r o(i, i)=r(3)$

18 continue

c

c

do $22 i=10.21$

$r b(i, i)=r(6)$

$x b(i, i)=x(16)$

22 contive

c

c

$r b(23.23)=r(4)$

$r b(22.22)=r(5)$

$x b(23.23)=x(13)$

$x b(22.22)=x(14)$

$x b(23,22)=x(15)$

$x b(22.23)=x(15)$

return

end

c

c

$c$

c

subrostine cnduct (vbovd)

inolicit real $\star 8(a-h \circ 2-z)$

dimension vb(24), vd(12) , icl(12)

common/blk200/icl

$v d(1)=v b(4)$

$v d(2)=-v b(6)$

$v d(3)=v b(5)$

$v d(4)=-v b(4)$

$v d(5)=v b(5)$

$v d(6)=-v \partial(5)$

$v d(7)=v b(7)-v b(8)$

$v d(8)=v b(7)-v b(9)$

$v d(9)=v b(8)-v b(9)$

$\operatorname{vd}(10)=v o(s)-v b(7)$.

$\operatorname{va}(11)=v b(9)-v b(7)$

$v d(12)=v b(9)-v b(8)$

c

c

do $1 J i=1.6$

$i_{0}=i+1$

$i q=i+2$

if $(i 0 . e a .7)$ i $0=1$

if $(i a \cdot e q .7) i q=1$

if (ia.eq. 8$) i q=2$

if $(i c(b) . e q \cdot 1$. and. $i c(s i 0) \cdot e q .1) \vee d(i a)=0.6$ 
10 continue

to $20 \quad i=7.12$

$i p=i+1$

$i q=\mathbf{i}+2$

if (io.ea.13)io=7

if (ia.eq.13)ia=7

if $\left(i a_{1} e a .14\right)$ i $a=8$

if $(i c((i) . e q .1$. and.icl(io).eq.1) vd(iq) $=0.5$

20 continue

return

end

subrostine cdscts(vbevds)

inolicit real $\star 8(a-h, j-2)$

timension vo(24),vd5(12)

$\operatorname{vas}(1)=-v b(6)$

$v J 5(2)=v o(5)$

$\operatorname{vd} 5(3)=-v 0(4)$

$v d s(4)=v b(6)$

$v d 5(5)=-v b(5)$

$v J 5(6)=v j(4)$

$v d 5(7)=v j(7)-v b(9)$

$v a 5(8)=v b(8)-v b(9)$

$v d 5(9)=v o(8)-v b(7)$

$v d 5(13)=v j(9)-v b(7)$

$\operatorname{vas}(11)=v o(9)-v b(8)$

$v d 5(12)=v b(7)-v b(8)$

return

end

c

$c$

c

c

subroutine initcn(icloanoano,mom2)

inolicit reat $\star s(a-h, 0-z)$

dimension icl(12), an(12), ano(12), cbran(12.12)

$1 \cdot \operatorname{rt}(12,12), x t(12,12) \cdot \operatorname{rn}(12,12), x)(12,12), y n(12,12)$,

$2 \mathrm{cb}(12,12), \operatorname{cbt}(12,12), j d(6), \operatorname{adum}(12) \cdot x \operatorname{lo}(12 \cdot 12)$,

3rnt $(12,12)$, yt $(12,12)$

commollblk2l cbran

comnos/blk4/yt,rtext

common/blks) xnerneyn

common/blk 7$)$ cb,cbt

common/blk8/ is

common/blk28/intv

common/blk13/int 1 ,int 2

common/blk29/intdenogond

common/olk30/nt jomt $j \cdot l t j \circ n s j \circ m s j \cdot l s j$

$\rightarrow 10=0.0$

$n \circ q=0$

$n=m 2-1$

$n 1=m-1$

to $113 \quad i=1.12$

110 adum $(i)=0.01$

do $101 i=1, m$

do $101 j=1, n$

$r n(j \cdot i)=0.0$ 


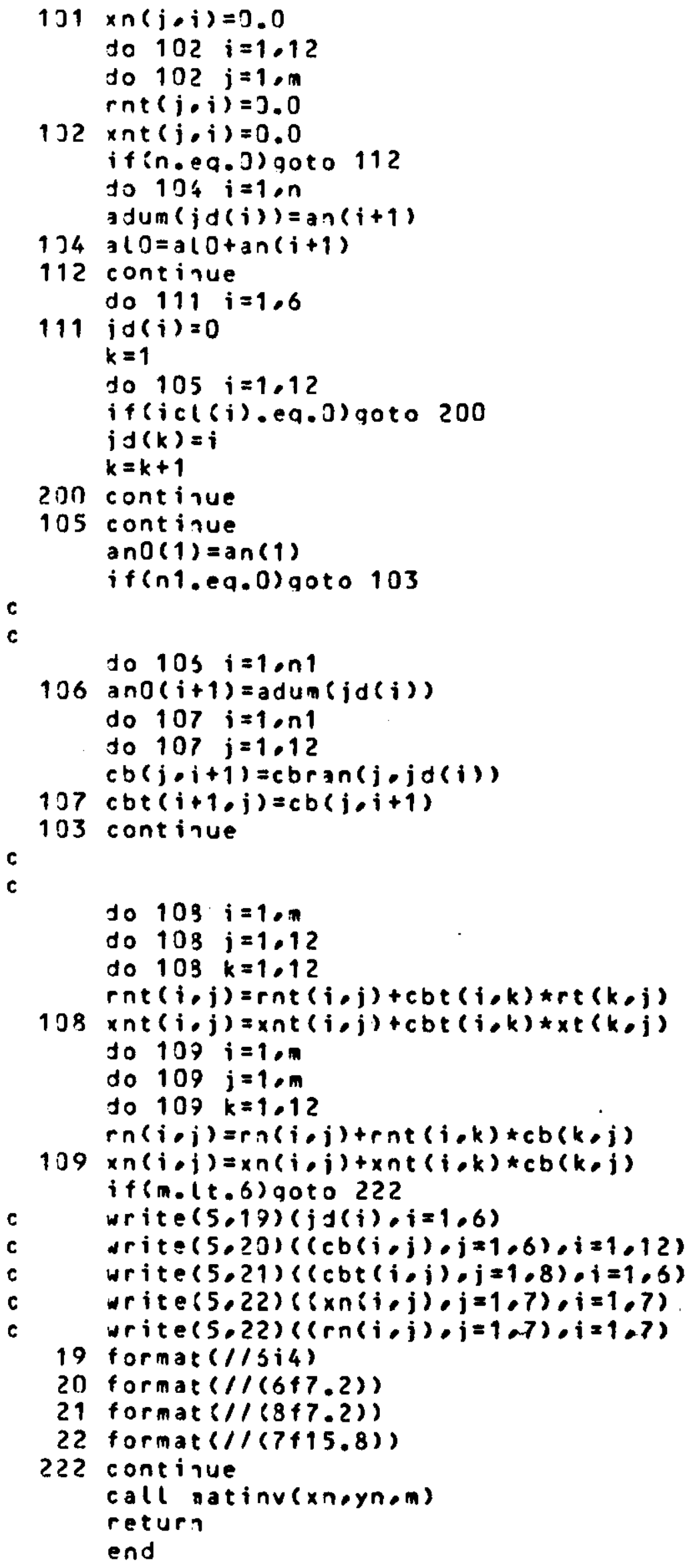


subroutine branvivb, $n)$

imolicit real $\star 8(a-h, 0-z)$

timension vb(24),ab(24), rb $(24,24), \times b(24,24)$,

1 a (24).94t(12).94(12).94b(24).ctran(24.12).ctrant (12.24),

$1 c b(12,12) \cdot \operatorname{cbt}(12,12) \cdot e(24), v i(12), v a(12)$

common/blk1/ctran

commor/blkg/ctrant

common/blk156/dildt

common/blk3/xborb

common/blk $7 / c b, c b t$

common/blk10/ 94

common/blk11/ab

common/blk21/e

common/blk22/vm

$e(4)=-0.5392 d+00 * e(1)$

$e(5)=-0.5392 d+00 * e(2)$

$=(6)=-0.5392 d+00 * e(3)$

$e(7)=-0.3113 d+00 * e(1)$

$e(8)=-0.3113 d+00 * e(2)$

$e(9)=-0.3113 d+00 * e(3)$

do $103 j=1.24$

$v o(i)=.0 j+00$

$q 4 b(i)=.0 d+00$

$100,(i)=.0 d+00$

to $101, i=1: 24$

do $101 j=1.24$

$101 a(i)=a(i)+r b(i, j) \star a b(j)$

do $102 \quad i=1.12$

$v m(i)=0.0$

$v i(i)=.0 d+00$

$102 q 4 t(i)=.0 d+00$

do $103 i=1.12$

Jo $103 \mathrm{j}=1 \mathrm{om}$

$13394 t(i)=q 4 t(i)+c b(i, j) * q 4(i)$

do $104 i=1.24$

do $104 j=1.12$

$134 \quad 74 b(i)=94 b(i)+c t r a n(i \cdot j) * 94 t(j)$

$d i t d t=94 b(24)$

do $105 \quad i=1.24$

to $105 j=1.12$

$106 v b(i)=v b(i)+x b(i, j) * 74 b(j)$

do $107 \quad i=1.24$

$107 v b(i)=-v o(i)-a(i)+e(i)$

do $105 \quad i=1.12$

do $105 \mathrm{j}=1.24$

$135 v i(i)=v i(i)+c t r a n t(i, j) \star v b(j)$

do $103 \quad i=1, \mathrm{~m}$

do $103 j=1,12$

$138 v n(i)=v m(i)+c b t(i, j) \notin v i(j)$

return

end

$c$

c

subroutine params

imolicit real $\star 8(a-q, 0-2)$

common/blk6/aloha,omeqa,vmaxeangle

common/blk15/alooacoh

common/blk26/haloohfrea 


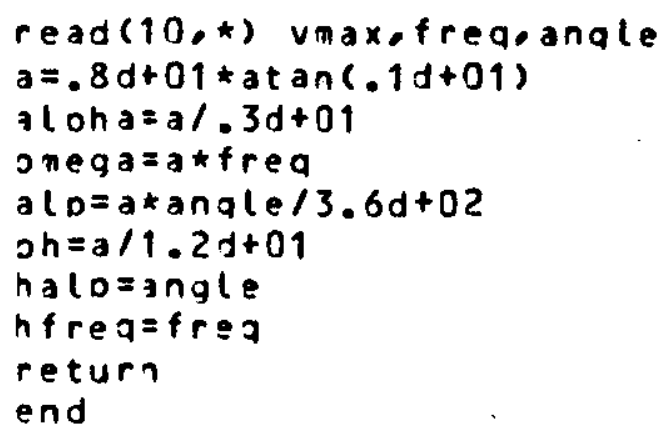




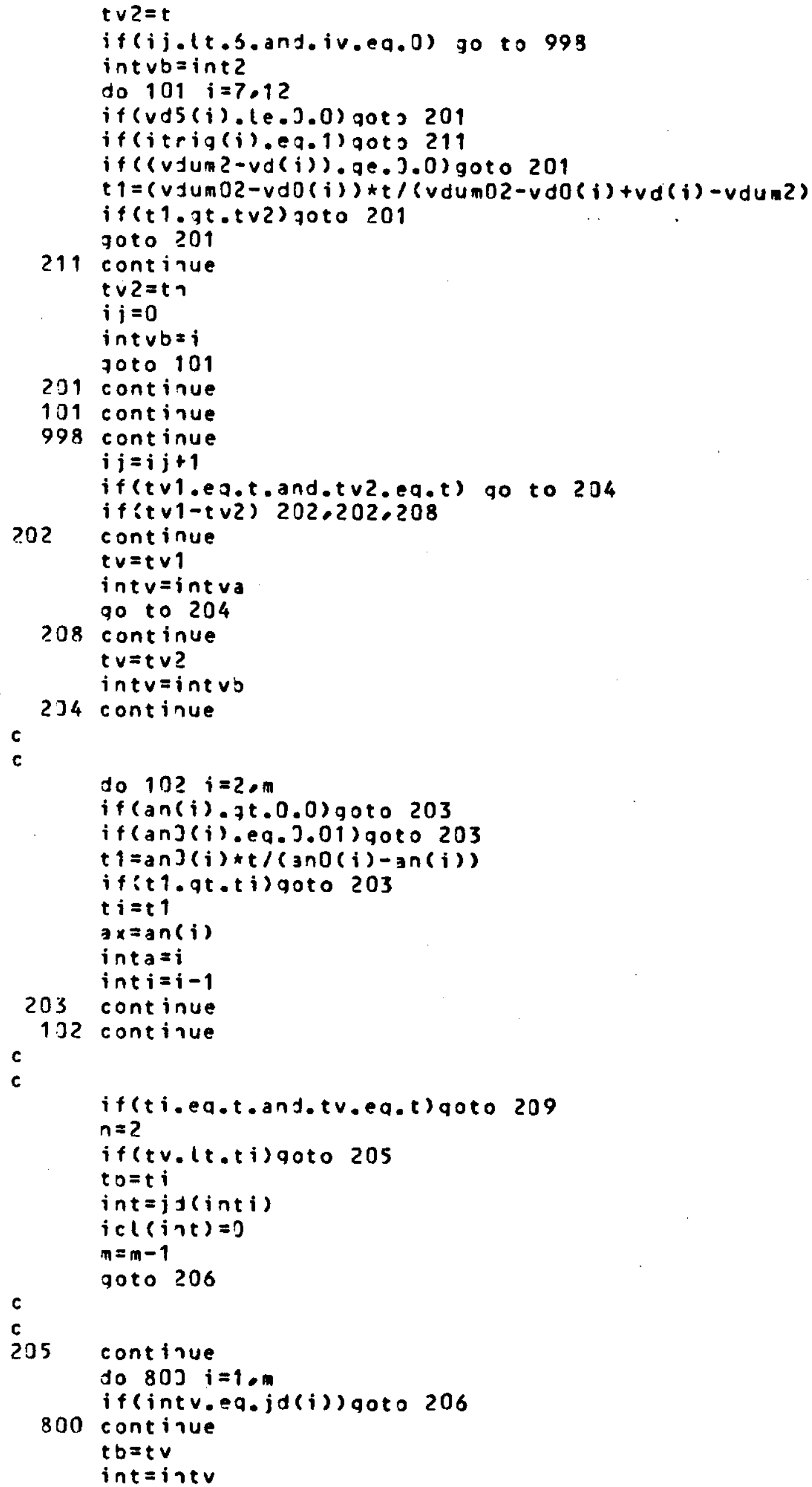




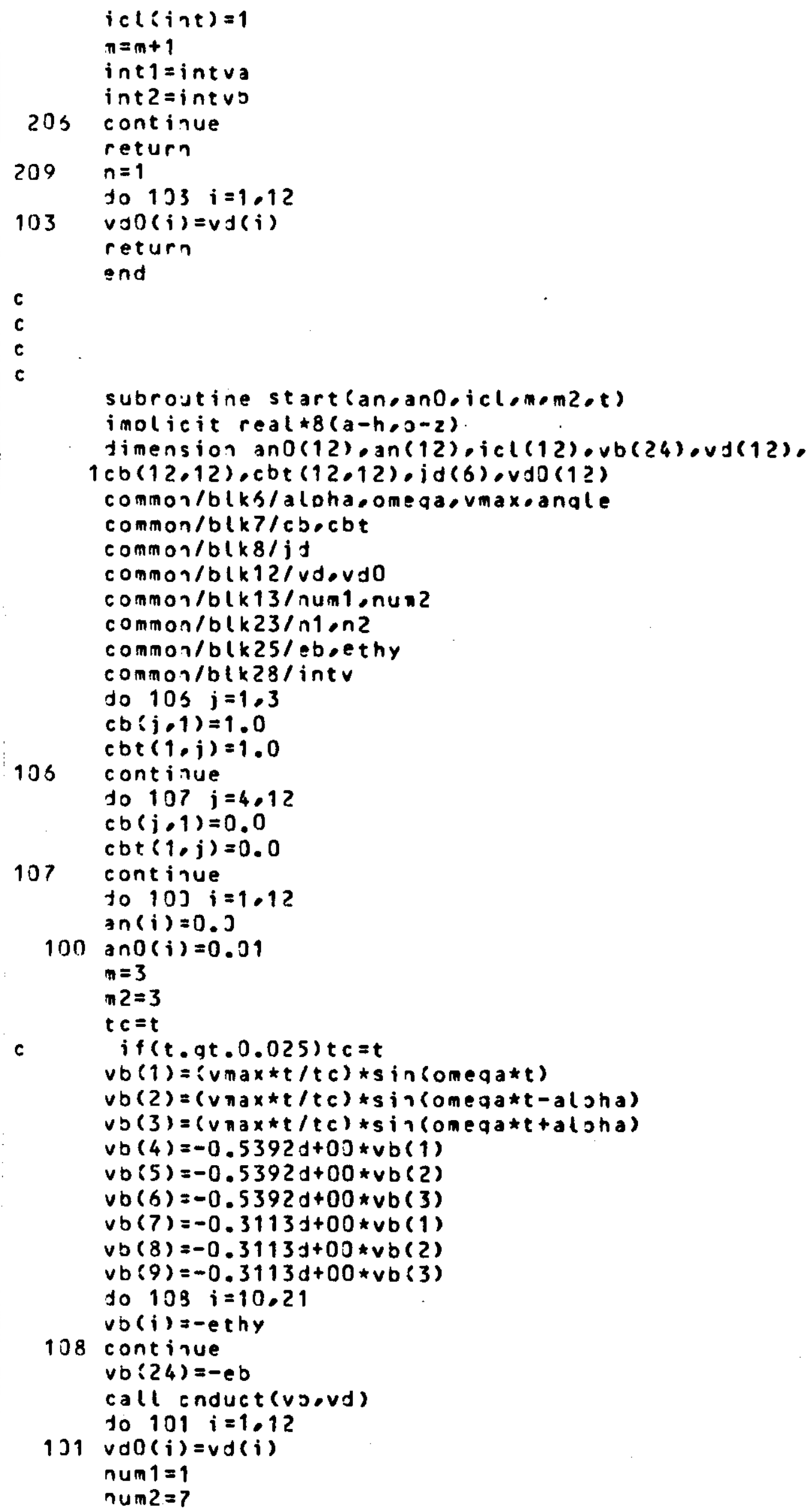




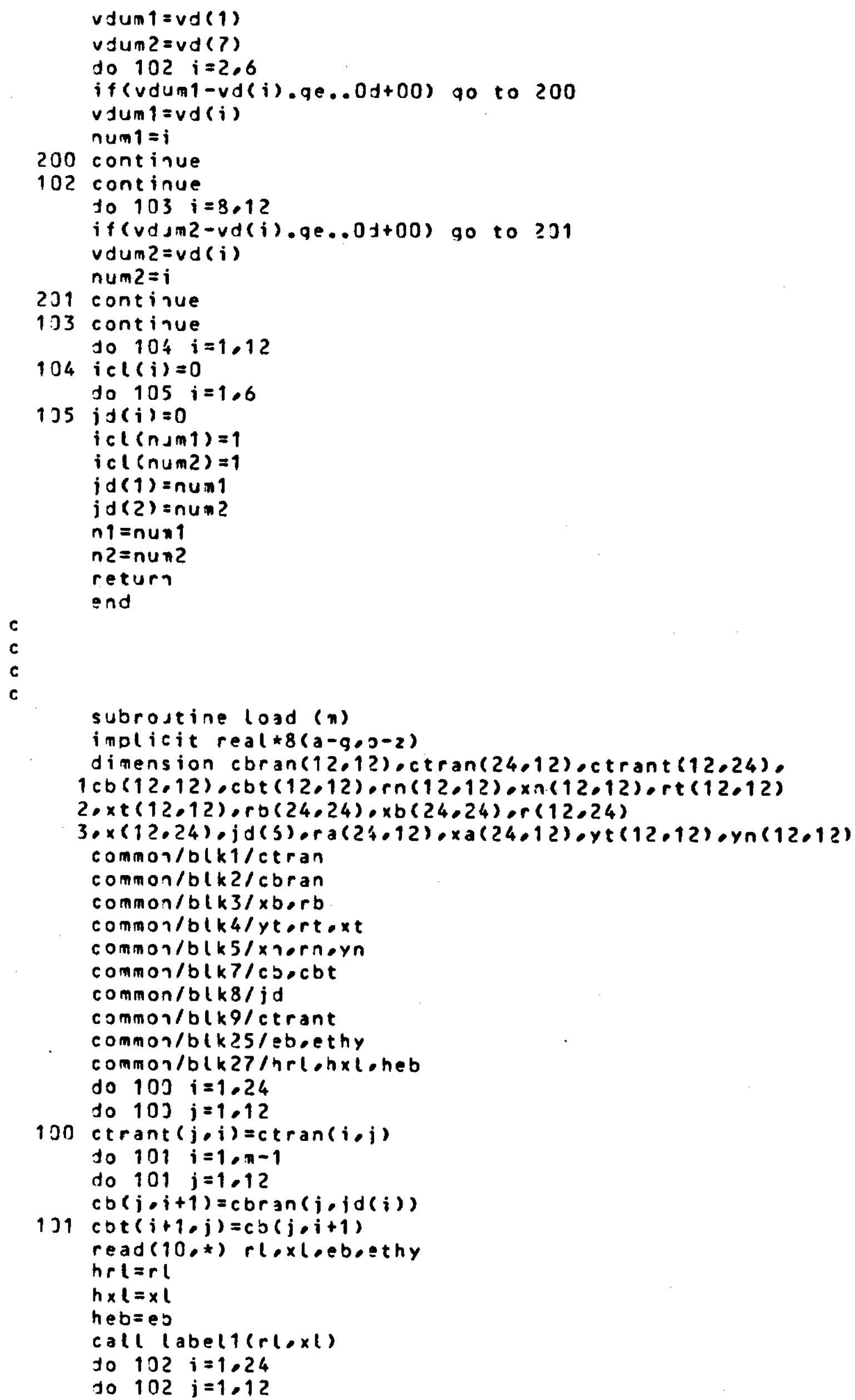




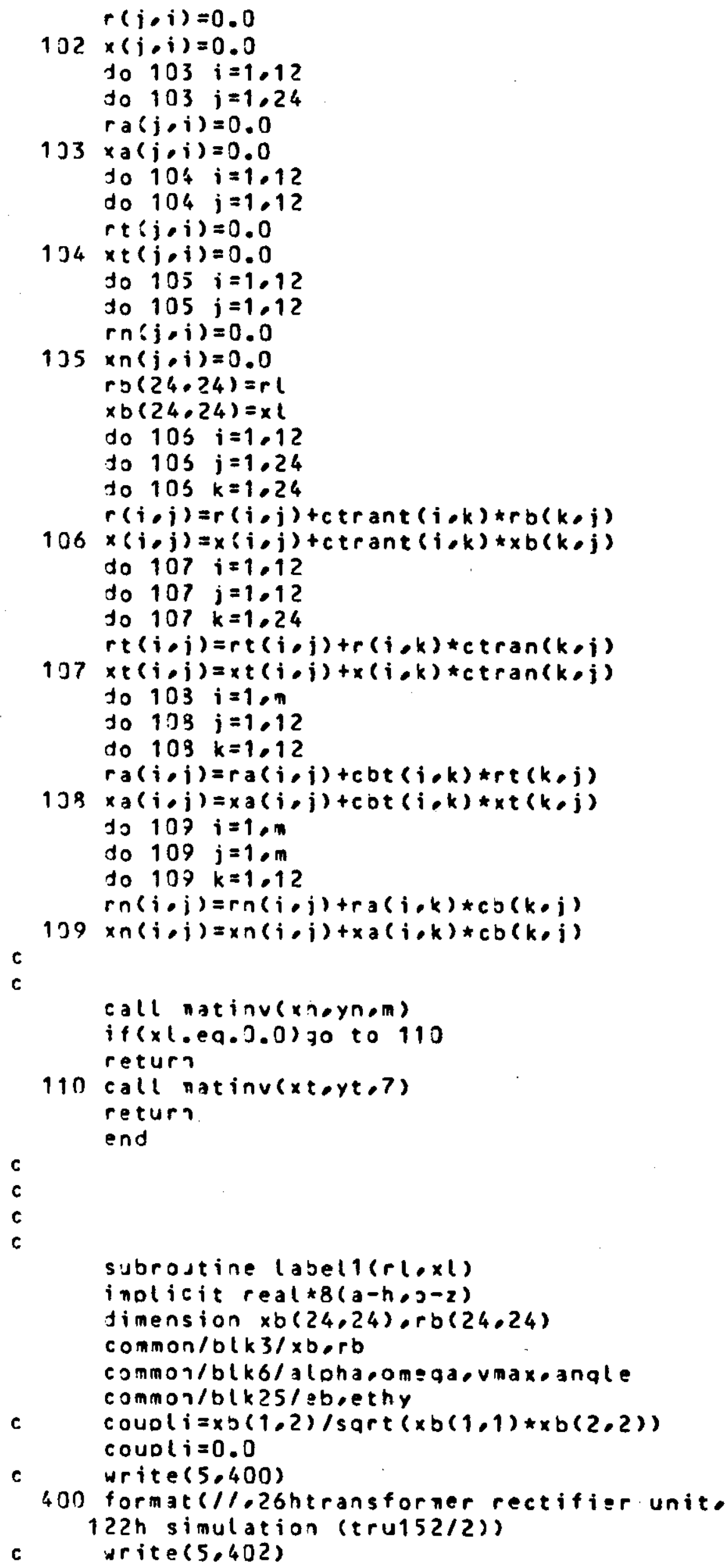




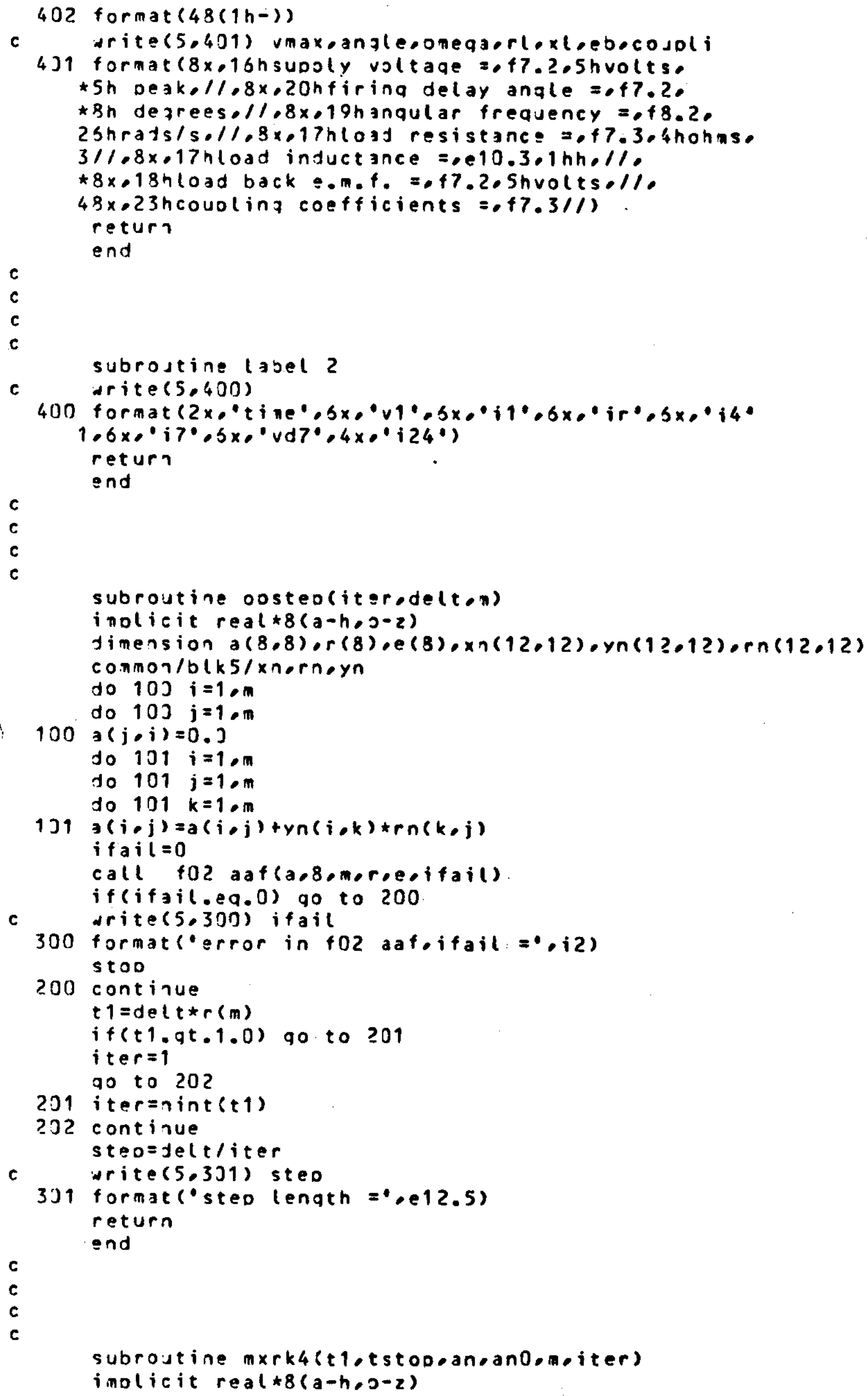




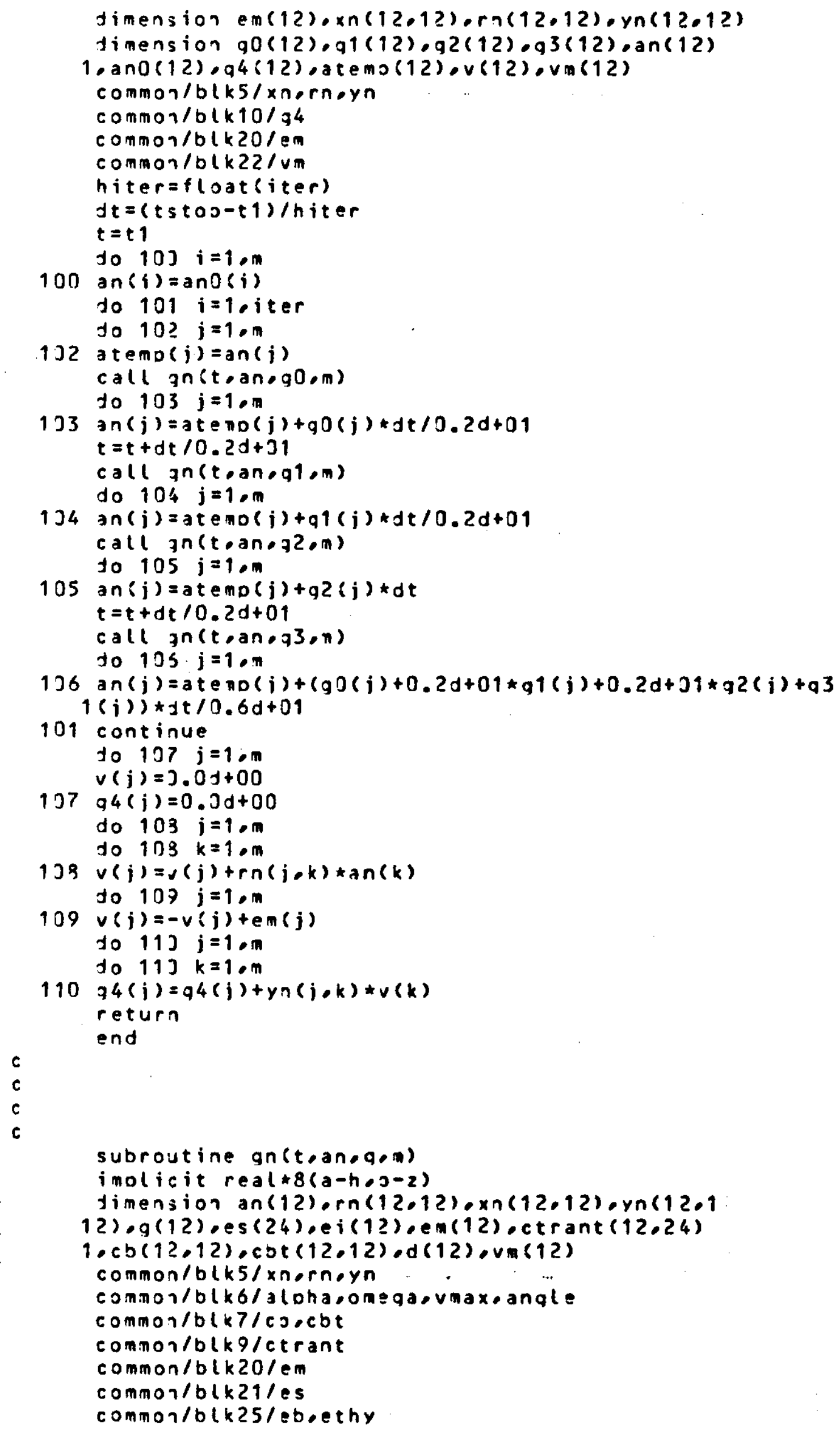




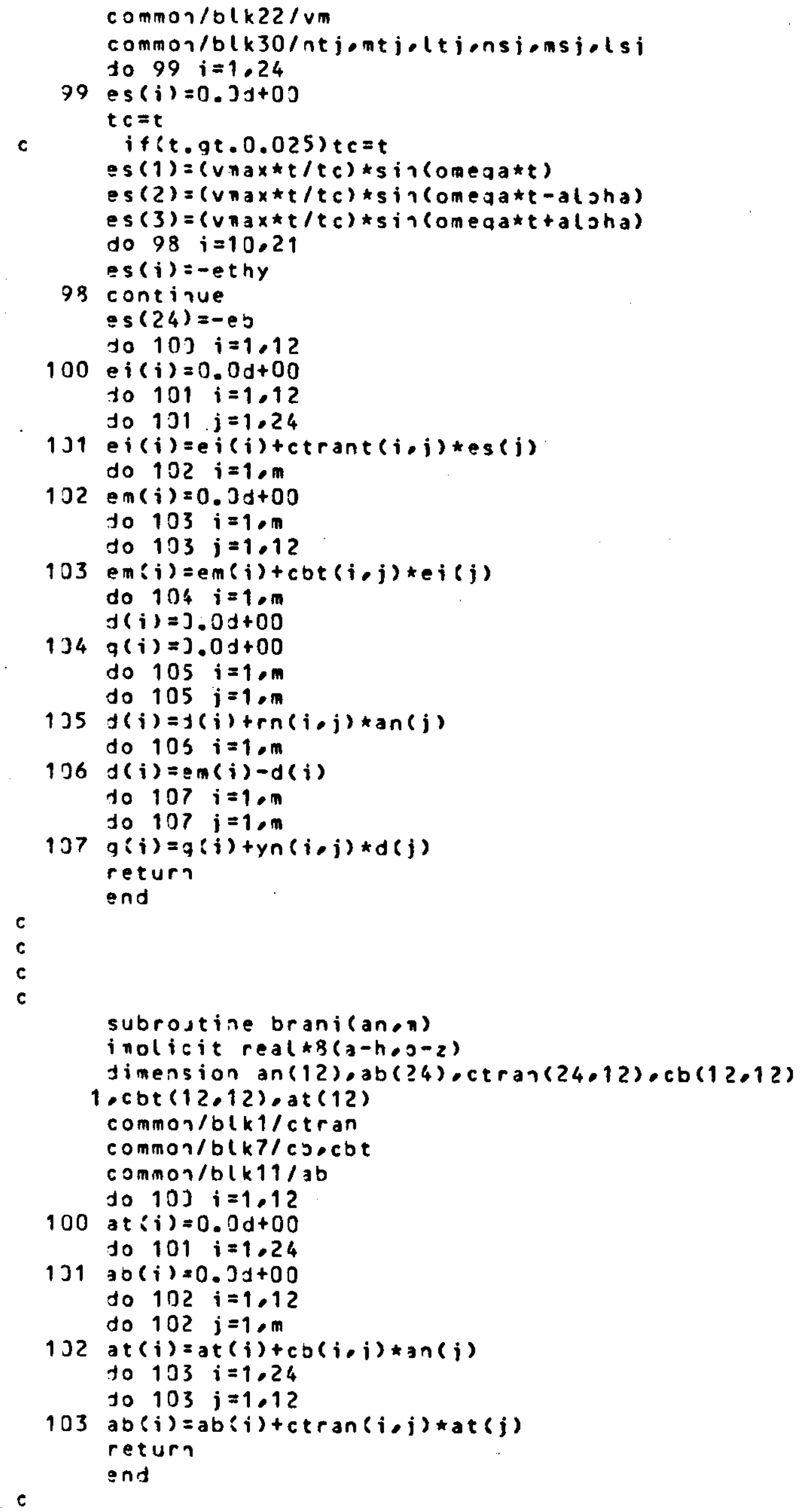


subrostine orint (tstoopaboitrig)

inoli=it real $* 3(a-h, 3-z)$

Jimension icl(12),vb(24), ab(24), itrig(13), ithy(12)

dimension $r b(24,24), \times b(24,24)$

common/blk3/ xborb

commor/blk156/ditdt

common /blk20j/icl

commor/blk521/vx

common/blk892/axear

commor/olk201/vb

common/blk433/ikeij

common/blk13/i1,i2

common/blk25/eboethy

commor/blk28/intv

do $401 i=1,12$

$i(h y(i)=0$

$i_{0}=i+1$

if (io.ea. 7$)$ io $=1$

if (io.ea.13)io=?

401 continue

iffict(i).eq.1.or.ic( (ip).eq.1)ithy(i)=1

c

c

if(icl(7).ea.1.or.icl(8).ea.1) go to 500

if (icl( 9$)$.eq.1.or.icl(10).eq.1) ao to 501

if(icl(11).ea.1.or.icl(12).ej.1) joto 502

30 to 503

$532 v x=v b(7)-v b(9)$

90 to 503

$501 v x=v b: 7)-v b(8)$

To to 503

$500 v x=-0.6$

533 continue

$a x=a b(4)-a b(5)$

$a r=a b(1)-a b(3)$

$c c$

$$
\text { write }(5,400) \text { ts }
$$

400 format $(+3.5,2(1 x, f 10.7), 5(1 x+f 9,3) \cdot 2(1 x+i 2), 1 x+12(1 x+i 1) \cdot 1 x \cdot 14(1$ li 11

return

end 


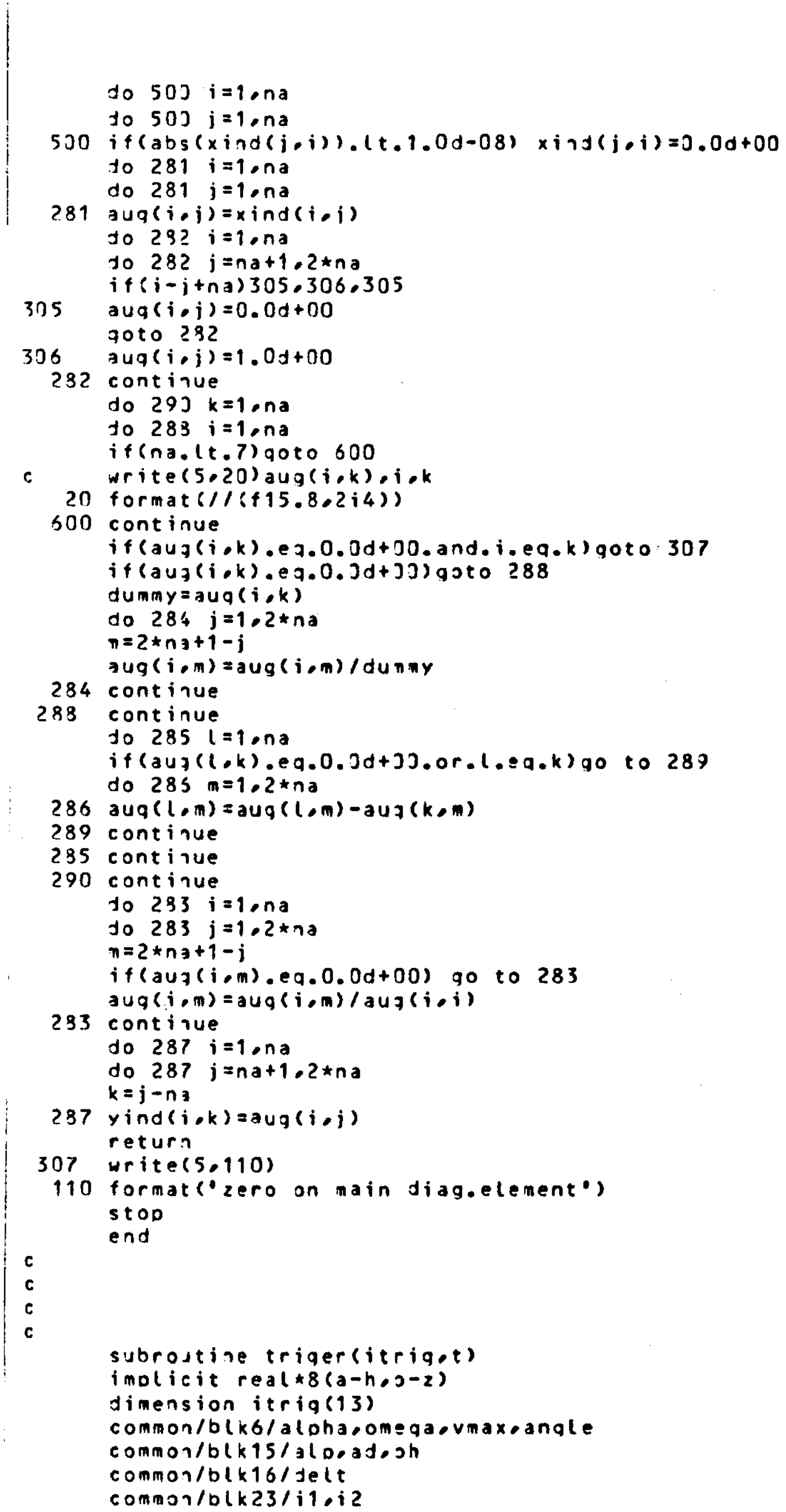




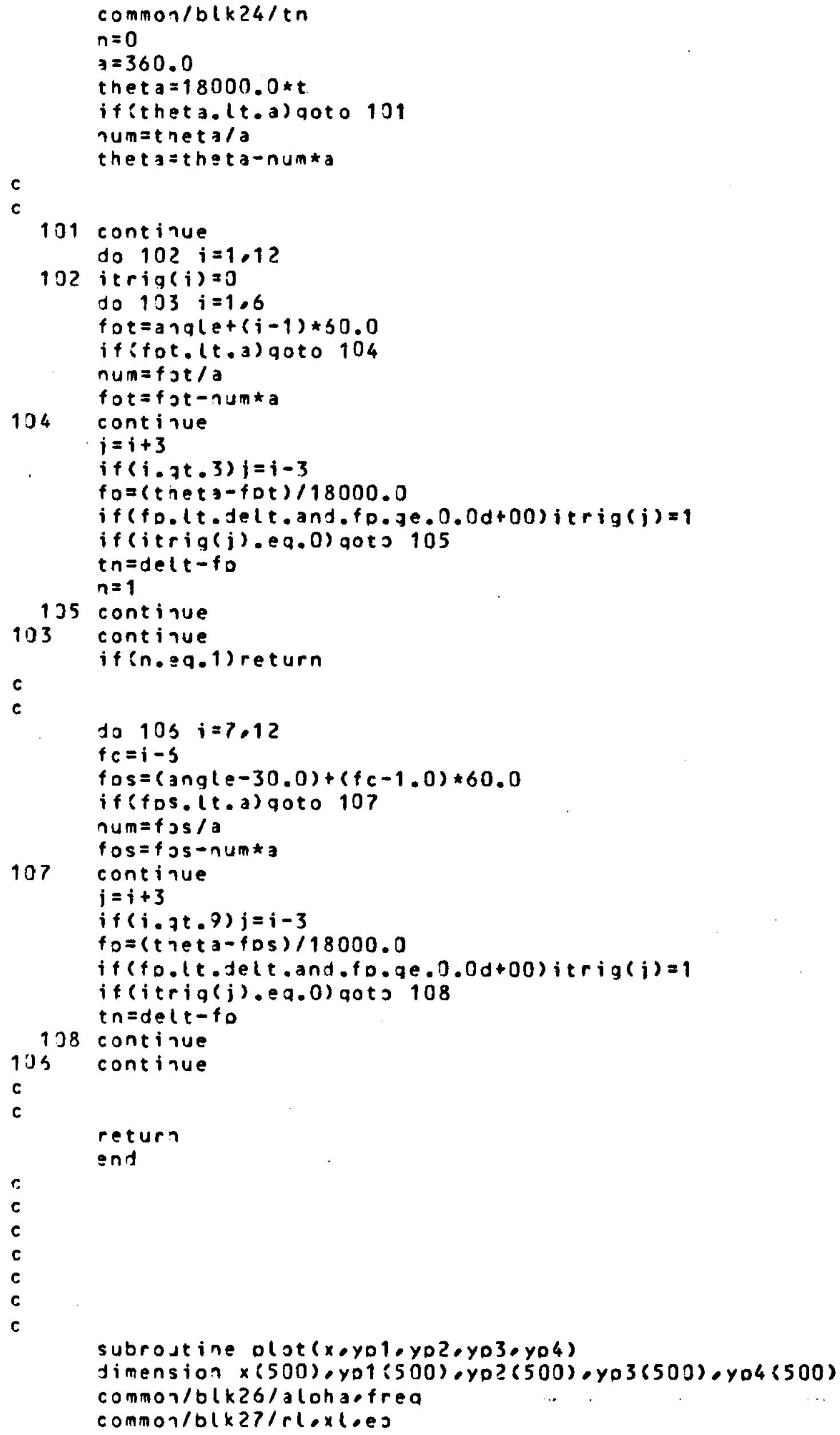


$s 1=15.0$

$y \max 1=y 01(1)$

$y \max 2=y 02(1)$

do $1 i=2,200$

if (ymaxi. (t. yo1 i i) ) ymax1=yo1(i)

if $(y m a \times 2 \cdot(t \cdot y o 2(i))$ yna $2=y 02(i)$

1 continue

$y \max =y \max 2$

$c$

if $(y m a x 1 . q t \cdot y n a \times 2)$ ymax $=y m a \times 1$

c

C

c

C

c

if (abs $(y n a x) \cdot a t .10 .0$. and abs $(y \max ) \cdot(e .30 .0) \mathrm{s} 1=30.0$

if $(\operatorname{abs}(y \operatorname{nax}) \cdot j t .30 .0$. and.abs $(y \max ) \cdot(e .60 .0) s 1=60.0$

if $(a b s(y n a x) . q t .60 .0$ and abs $(y \max ) \cdot(e .120 .0) s 1=120.0$

if (abs $(y \max ) .9 t .120 .0$. and.abs $(y \max ) \cdot(e .300 .0) s 1=300.0$

if $(a b s(y \max ) .9 t .300 .0$. and.abs $(y \max ) \cdot(e .600 .0) s 1=600.0$ $s 2=s 1+s 1 * 2: 0 / 3.0$

$s 3=s 1+s 1 / 3.0$

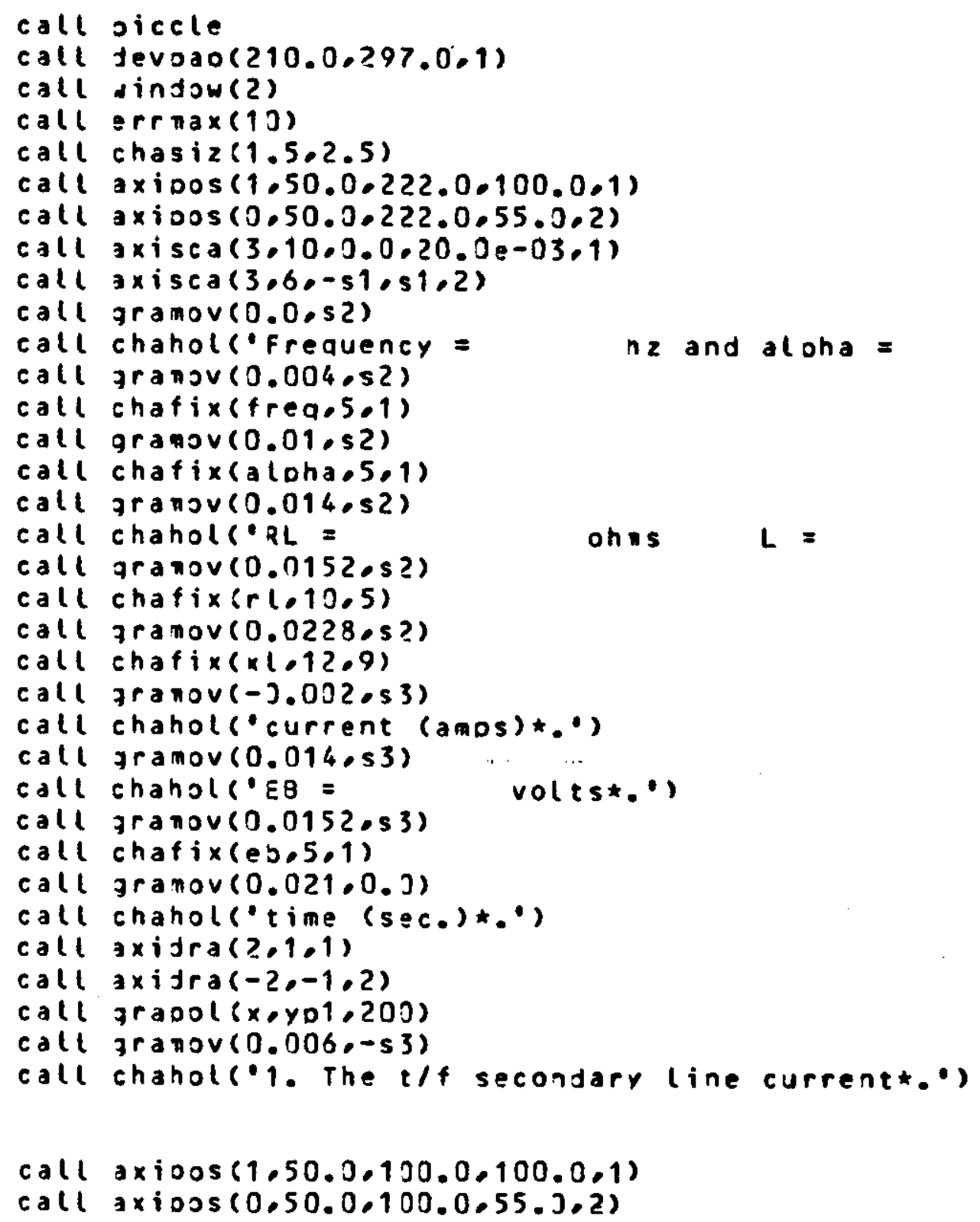

call axioos $(1,50.0,100.0,100.0 .1)$

call axioos $(0.50 .0 .100 .0 .55 .3 .2)$ 
$\operatorname{call} 3 \times \operatorname{sisca}(3,10,0.0 \cdot 20.0 \mathrm{e}-03,1)$

call $3 \times$ isca $(3,6,-51,51,2)$

call gramov $(-0.002 .53)$

call chahol("current (amos)*..')

call zranov $(0.021 .0 .3)$

call chahol $\left({ }^{\circ}\right.$ time $\left.\left(s=c_{0}\right) *_{0}{ }^{\circ}\right)$

call $a x i j r a(2,1,1)$

call axidras $(-2,-1,2)$

call zraool $(x . y 02,20))$

call zranov $(0.006,-53)$

call chahol('?. The $t / f$ tertiary winding current*. $\left.{ }^{\circ}\right)$

c

c

c

call axioos $(1.50 .0 .100 .0 .100 .0 .1)$

call $3 \times i 00 s(0.50 .0 .100 .0 .63 .0 .2)$

call $3 \times$ isca $(3,10,0.0 \cdot 20.0 e-03.1)$

call $3 x$ isca $(3,8,-200,0,200,0,2)$

call aramov $(-0.002,225.0)$

call chahol ("voltage (volts)*.")

call granov $(0.021 .0 .3)$

call chahol( time (sec.)*.*)

call axidra(2,1,1)

call axjJra( $(-2,-1,2)$

call graool $(x \cdot y 03,20)$

call Jranov $(0.006,-200.0)$

call chahol('3. thyristor voltage - $\left.1 r^{*} .^{\circ}\right)$

calt $3 \times$ ioos $(1.50 .0 .15 .0,100.3 .1)$

call $3 \times i \cos (1,50.0,15.0 .34 .0 .2)$

call axisca $(3,10,0.0,20.0 \mathrm{e}-03,1)$

call $3 \times$ isca $(3,4,0.0 .200 .0 .2) \ldots$

call jranov $(-0.002 .225 .0)$

call chahol("voltage (volts)*.")

call jranov $(0.021 .0 .3)$

call chahol( time (sec.)*..")

call axidra $(2,1,1)$

call axidra( $(-2,-1,2)$

call graool $(x \cdot y 04.20))$

call zranov(0.006.-75.0)

call chahol $6^{\circ} 4$. load voltage*. $\left.{ }^{\circ}\right)$

retur?

end

subrostine olot $1(x, y 01, y 02, y 03, y 04)$

Jimension $x(500)$,yol $(500)$,yol $(50)$, yo $3(50)$, yo $4(500)$ common/blk26/alohapfrea common/blk27/rloxloeb

$s 1=15.0$

$y \max 1=y p 1(1)$

yna $\operatorname{ma}=y 02(1)$

do $1 i=2,200$

if $(y \max 1 .(t . y 01(i))$ ynax $1=y 01(i)$

if $(y \pi a \times 2,(t, y 02(i))$ ynaxz $=y p 2(i)$

1 continue 
$y \max =y \max 2$

if $(y m a x 1 . g t, y n a \times 2)$ ym $3 x=y n a \times 1$

c

if $(a b s(y \pi a x)$. at 10.0 . and abs $(y \max ) \cdot(t .30 .0) s 1=30.0$

$i f(a b s(y \max ) . q t .30 .0$. and.abs $(y \max ) .(t .120 .0) s 1=120.0$

if $(\operatorname{abs}(y \max )$. at. 120.). and.abs $(y \max ) \cdot(t .300 .0) s 1=300.0$

if $\left(a b s\left(y \pi x_{x}\right)\right.$. Jt. 300 . ). and.abs $\left.(y \max ) .(t .60) .0\right)$ s $1=600.0$

$s 2=s 1+s 1 * 2.0 / 3.0$

$s 3=s 1+s 1 / 6.0$

c

c

call oicele

call Jevoap(250.0.297.0.1)

call dindow (2)

call ermax (10)

call chasiz (1.5.2.5)

call $3 \times$ ioos $(1.50 .0 .222 .0 .100 .0 .1)$

call axioos $(0.50 .0 .222 .0 .55 .3 .2)$

call axisca(3.10.0.0.20.0e-03.1)

call axisca $(3,6,-51,51,2)$

call Jramov $(0.0 .52)$

call chahol ("Frequency =

call Jranov $(0.004 .52)$

call chafix(freq.5,1)

call 7ramov $(0.01 .52)$

call chafix(aloha.5.1)

call zranov $(0.014 .52)$

call chahol ${ }^{\circ}$ RL $=$

call gramov(0.0152.s2)

call chafix(rl, 10,5$)$

$c a l l$ Jranov $(0.0228 .5 ?)$

call chafix $(x l, 12,9)$

call jranov $(-0.002 .53)$

call chahol('current (amps) $\star_{0}^{*}$ )

call aranov $(0.014 .53)$

call chahol C EB =

call zranov(0.0152.53)

call chafix $(e b, 5,1)$

call gramov $(0.021 .0 .0)$

call chahol('time (sec.)*..')

call $\rightarrow x i j r a(2,1,1)$

call $3 x i j r a(-2,-1,2)$

call zraool $(x, y 01,203)$

call Jranov $(0.006,-53)$

call chahol("1. The suody line current*."')

c

c

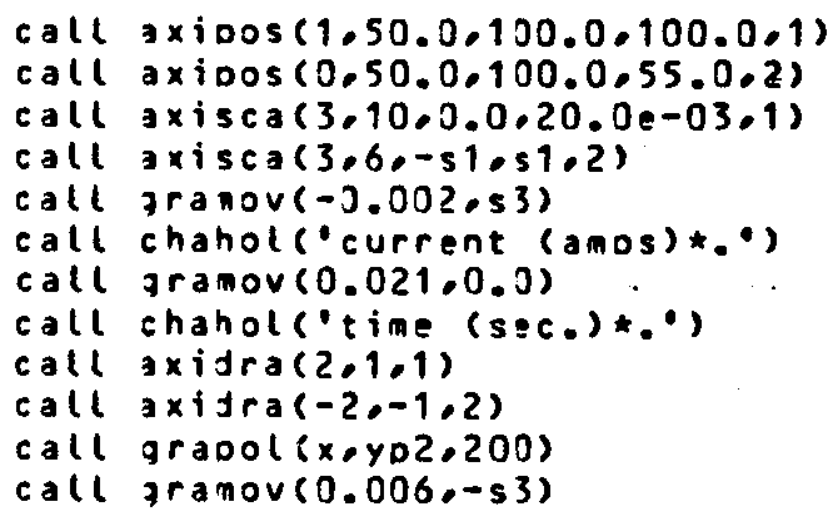




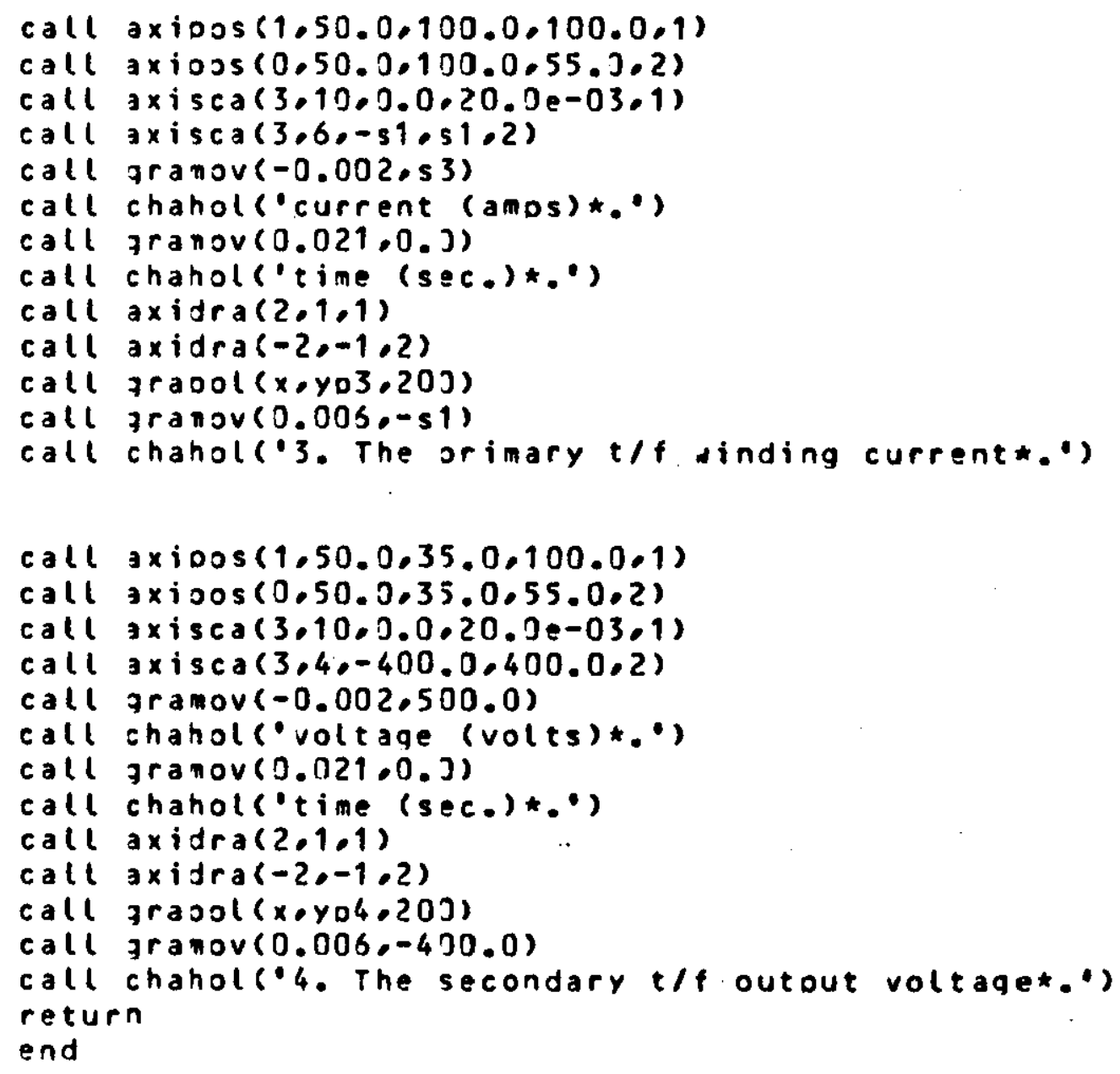




\section{DATA-16}

$$
\begin{aligned}
& \begin{array}{llllll}
0.708 & 0.289 & 0.307 & 0.0 & 0.0 & 0.1
\end{array} \\
& \begin{array}{lllll}
1.66 & 0.5131 & 0.523 & 0.506 & 0.17
\end{array} \\
& \begin{array}{lllll}
c .279 & 0.0 & 0.0 & 0.0 & 0.0
\end{array} \\
& 0.0 \quad 0.0 \quad 0.0 \quad 0 . n \text { n.? }
\end{aligned}
$$

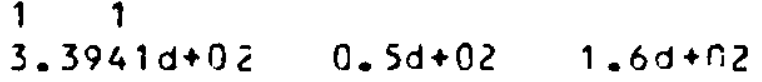


imolicit real $\star 8(a-h, 0-w, 2)$

dimension ano(12),an(12),ab(24),vb(24),ic( (12),

1vd(12),vdo (12) , rb (24,24),itria(13)

dimension cbran $(12,12) \cdot v(24), c \times(2100)$

dimension $c 1(2100), c ?(2100), c 3(2100), c 4(2100)$

dimension ithy(12),ci1(30),ci2(30),col:30),co2(30)

dimension $t i 1(30), t i 2(30) \cdot \operatorname{tol}(30) \cdot \operatorname{to} 2(30) \cdot \bmod 1(30) \cdot \bmod 2(30)$

real $* 3$ intvexb(24.24), xl $\times$ nn $(12,12), \times n(12.12)$

real $* 3$ ynl $(30), y m 2(3)$, yml(3)

dimension $r n(12,12)$, ay $1(2100)$, ayz $(2100)$, ay $(2100), v d l(2100)$ common/blk438/ik,ij

common/blk?/coran

comnor/blk3/xo,rb

common/blks/xnerneyn

common/blk156/dildt

commoz/blks21/vx

common/blk11) ab

common/blk12/vdevdo

common/blk13/id1,id2

common/blk892/axear

common /olkzoj/icl

common/blk201/vi

common/blk33/itria

common/blk16/jelt

commor/blk24/tn

common/blk25/ebethy

common/blk6/alohacomeqaeangle

common/blk36/nstudeicn

common/blk30/frezovmax

common/blk31/rloxl

common/blk32/ttet 1

commol/blk41/cx,cl,c2,c3,c4

common/blk43/ost.cst

common/blk45/dxs

common/blk50/numb

common/blk61/ia

common/b(k53)ak

common/blk $34 / n k$ 
common/blk46/ti1,ti2,to1,to2

common/blk47/int

common/blk48/nod 1 mot 2

common/blk51/ci1,ci2,co1,co2

common/blk49/ithy

common/blk60/ay1,ay2,aylevdt

common/blk66/ym1,ym2,ymlerlx

common/btk70/st 1,dt2,dt3,dt 4

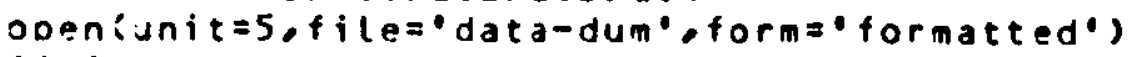

$i j=1$

$i k=1$

it $n=1$

C

C

C

The zob natrix is set by subroutine const

the $r$ aquired input data is obtained

vmax - Deak suody voltaqe

frea - supoly frequency

eb - load back enf

ethy - thyristor treshold voltaje when conducting

$r$ - load resistance

$x l$ - load inductance

nstud - number of studies

tim - time in seconds

delt - step lenght

dt $1, d t 2, d t 3, d t 4$ - deviation in deqrees for the triquer oulst firing delay angle is obtained from subrostine params

call consts

read:5,k) vmax,frea,eb,ethy,rl, xl

read $(5, *)$ nstudetimedelt

read $(5, *) d t 1, d t 2, d t 3, d t 4$

to 10 s icnt $1=1$ enstud

$i \mathrm{t} r=1$

icn=icnt 1

call oarans

inum $=1$

$t=1.0 y-09$

ts t OD $=t+$ det $t$

call start(an,annoicl,m,m2,t)

num $=t i m / d e l t$

call load $(\mathrm{m})$

call opsted (iterodelt,m)

Jo 107 icnt $3=1$, num

call nxrk4(tetstodoaneanoomeiter)

call triger(itrigetstoo)

i $v=0$

call intero(an,ano,icl,tbodelt,mem2,n,iv)

if (n.ea. 1) qoto 202

int $v=d e t t-t b$

it $r a D=1$

$t s=t$

$t i=t+t b$

$i e=0$

if (tn.te.tb)qoto 204

$t n=t n-t 0$

$i v=1$

204 continue

call nxrk4(tsetioan,ano,nzeiter) 
reoeated interoolation

to deternine as close as oossible the mesh current to the zero value

al so to deternine tha conduction interval and the variods commutations.

if (nk.ea.1)goto 106

if (ia.eq.j.or.t.(t.0.1050)goto 138

$i f(i n t . e a .8) \operatorname{col}(i(n)=t i-0.1050$

$i f(i n t . e a .2) \cos (i(n)=t i-0.1050$

if (id1.gt.1.or.t.qt.j.1251)qoto 109

if $(i n t .9 t .6) t o 1(i(n)=t i-0.1050$

$i f(i n t .(t .7) t 02(i c n)=t i-0.1050$

109 continue

if (ie.gt.6)qoto 105

if $(a n(i a) \cdot q t .0 .0$. and.an $(i a) .(t .0 .1 d-08)$ qoto 105

callextrao(tstooetioan(ia))

$i e=i e+1$

$30 t 0204$

135 continue

$\operatorname{an}(i a)=0.0$

goto 108

106 continue

if $(i n t . e a .7) c i 1(i c n)=t i-0.1050$

$i f(i n t . e j .1) c i 2(i c n)=t i-0.1050$

if (id1.gt.1.or.t.gt.).1251)goto 1.08

if (int.gt.s) goto 110

$t i 2(i(n)=t i-0.1050$

$\operatorname{nod} 2(i(n)=m-1$

qoto 111

$110 t i 1(i c n)=t i-0.1050$

$\bmod 1(i c n)=m-1$

111 continue

108 continue

call orani(an,mz)

call oranv(vbomz)

call initcnicicleaneano,mem2)

call cnduct ( $v b \circ v j)$

to $104 i=1.12$

$134 v d O(i)=v d(i)$

call orint (tioaboitr)

call nxrk4(tiotstoo,anoano,meiter)

call intero(aneano,icletbeintvememzeneiv)

if (n.:9.1)goto 202

int $v=$ int $v-t 0$

itrad $=i \operatorname{tad} a+1$

$\operatorname{tn}=0.0 \mathrm{~d}+00$

$i v=0$

$t s=t i$

$t i=t i+t b$

ifitrap.(t.5) qo to 204

write $(3,300)$

stoo

202

contijue

call orint (tstop,ab,itr)

do $101 i=1, m$ 


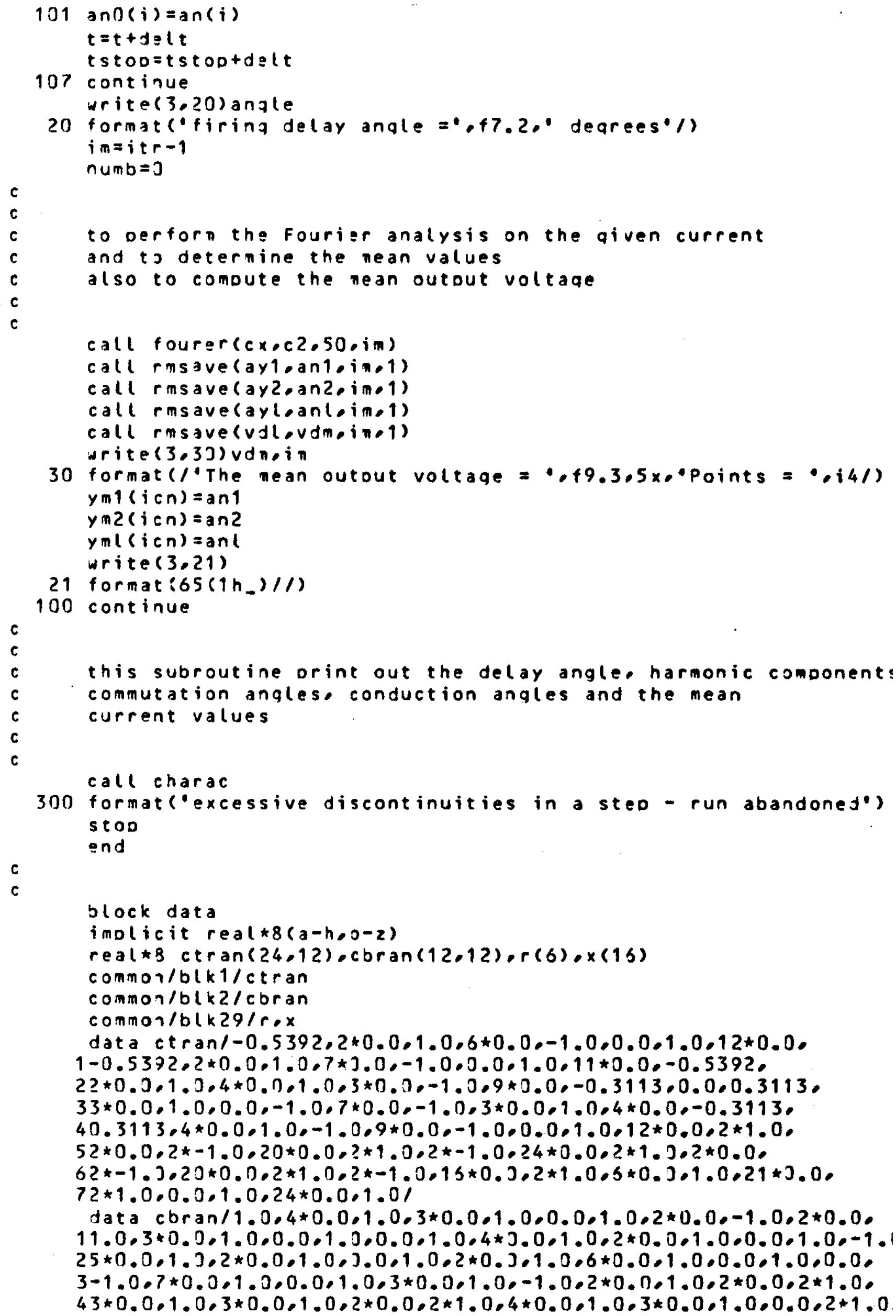

$c$ 


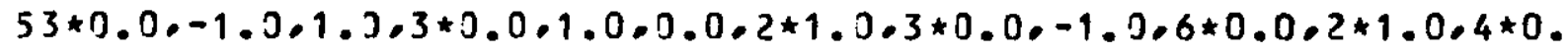
$6-1.0 .5 * 0.3 .2 * 1.01$

C

$c$

C

C

C

the transformer oaraneters

data $r / 0.708 .0 .289 .0 .307 .2 * 0.0 .0 .1 /$

data $\times / 1.66,0.901 .0 .523,0.505,0.17 .0 .279 .10 * 0.01$

end

subrostine consts

inolicit real $\star 8(a-h, 0-2)$

dimension $x b(24,24) \cdot r b(24,24) \cdot r(3) \cdot x(16)$

commor/btk $3 / \times b, r b$

common/blk?2/r,x

do $203 i=1,6$

write:3.600) ier(i)

600 format $\left(10 x, r^{\circ}, i 1,0=012.5\right)$

200 continue

do $201 i=1.16$

write $(3.601)$ i.x(i)

601 format $\left(10 x, x^{\circ}, i 20^{\circ}=0,12.5\right)$

$2 J 1$ continue

to $103 i=1.24$

do $103 j=1.24$

$r o(j \cdot i)=0.0$

$100 \times b(j, j)=0.0$

to $11 \quad i=1,3$

do $10 j=1.3$

$10 \times b(i, j)=x(7)$

$x b: j, i)=x(1)$

$r b(i, i)=r(1)$

do $12 j=4.6$

$12 \times 0(i-j)=\times(8)$

$i 1=i+3$

$x b(i-i 1)=x(2)$

do $13 j=7.9$

$13 \times b(i, j)=x(9)$

$i 1=i+5$

$x b(i, i 1)=x(3)$

11 continue

c

c

$15 \times b(i, j)=\times(8)$

do $14 \quad i=4.6$

do $15 j=1.3$

$\mathbf{i} 1=\mathbf{i}-\mathbf{3}$

$x b(i-i 1)=x(2)$

do $16 j=4.6$

16

$x b(i \cdot j)=x(10)$

$x b(i, i)=x(4)$

$r b(i, i)=r(2)$

do $17 j=7.9$

$17 \quad \times b(j, j)=x(11)$

$i 1=i+3$

$x b(i, i 1)=x(6)$

14 continue

c 
do $19 \quad j=7.9$

$19 \quad \times b(i, j)=\times(9)$

$i 1=i-5$

$x b(i, i 1)=x(3)$

$20 \quad \begin{aligned} & \text { do } 20 j=4,5 \\ & x b(j, j)=x(11)\end{aligned}$

$i 1=i-3$

$x b(i, i \uparrow)=x(6)$

21

to $21 j=7.9$

$x b(i, j)=x(12)$

$x b(i, i)=x(5)$

18

$r b(i, i)=r(3)$

c

c

continue

do $22 i=10.21$

$r b(i, i)=r(6)$

$x b(i, i)=x(16)$

22 continue

c

C

$$
\begin{aligned}
& r b(23,23)=r(4) \\
& r b(22,22)=r(5) \\
& x b(23.23)=x(13) \\
& x b(22.22)=x(14) \\
& x b(23.22)=x(15) \\
& x b(22.23)=x(15) \\
& \text { return } \\
& \text { end }
\end{aligned}
$$

c

c

c

c

subrostine cnjuct (vb.vd)

imolicit real $\star 8(a-h, 0-z)$

dimension vo( 24$)$,vd (12), ic ( $(12)$

common/blk200/icl

$v d(1)=v b(4)$

$v d(2)=-v b(6)$

$v d(3)=v b(5)$

$v d(4)=-v b(4)$

$v d(5)=v b(6)$

$v d(6)=-v b(5)$

$v d(7)=v b(7)-v b(8)$

$v d(8)=v b(7)-v b(9)$

$v d(9)=v b(8)-v b(9)$

$v d(10)=v o(3)-v b(7)$

$v d(11)=v b(9)-v b(7)$

$v d(12)=v o(9)-v b(8)$

c

c

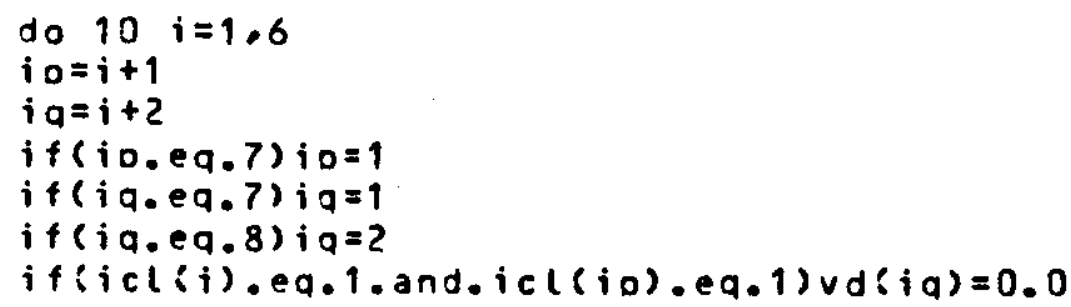


10 continue

to $20 \quad i=7.12$

$i p=i+1$

$i q=j+2$

if $($ io.ea.13) io $=7$

if $(i a . e q .13) i a=7$

if $(i a . e a .14) i a=8$

$i f(i c l(i)$.eq.1.and. $i c((i 0) \cdot e q \cdot 1) \vee d(i q)=0.0$

20 continue

retur?

end

C

c

C

C

subrodtine cduct5(vo.vd5)

imolicit real*8(a-h,o-z)

dimension $v b(24) \cdot v d 5(12)$

$v d S(1)=-v b(6)$

$v d 5(2)=v \supset(5)$

$v d 5(3)=-v b(4)$

$v d 5(4)=v b(6)$

$v d 5(5)=-v b(5)$

$v d 5(6)=v j(4)$

$\operatorname{vas}(7)=v b(7)-v b(9)$

$v d 5(8)=v b(8)-v b(9)$

$v d S(9)=v s(8)-v b(7)$

$v d 5(1)=v v(9)-v b(7)$

$v d 5(11)=v s(9)-v b(8)$

$v d 5(12)=v b(7)-v b(8)$

return

end

c

C

c

c

subrostine initen(ictoan,ano,mom?)

inolicit real $\star 8(a-h \cdot s-z)$

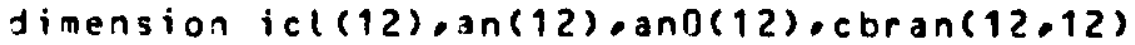

$1 \cdot \operatorname{rt}(12,12), x t(12,12) \cdot \operatorname{rn}(12,12), x+(12,12) \cdot$ yn $(12,1$

$22), c b(12,12), c b t(12,12), j d(6), a d u m(12), x n t(12$,

$312) \cdot r 3 t(12.12) \cdot y t(12.12)$

commol/blk?/ cbran

comon/blk4/ytertext

common/olks/ xnerneyn

common/blk?/ cbocbt

commol/blk8) jd

common/blk2s/intv

common/blk13/int 1 , int 2

$n=m 2-1$

$n 1=m-1$

do $113 i=1,12$

110 adum $(i)=0.01$

do $101 i=1, m$

do $101 \mathrm{j}=1, \mathrm{~m}$

$r n(j, i)=0.0$

$131 \times n(j, i)=0.0$

do $102 j=1,12$

to $102 j=1, m$

$\operatorname{rnt}(j \cdot i)=0.0$ 


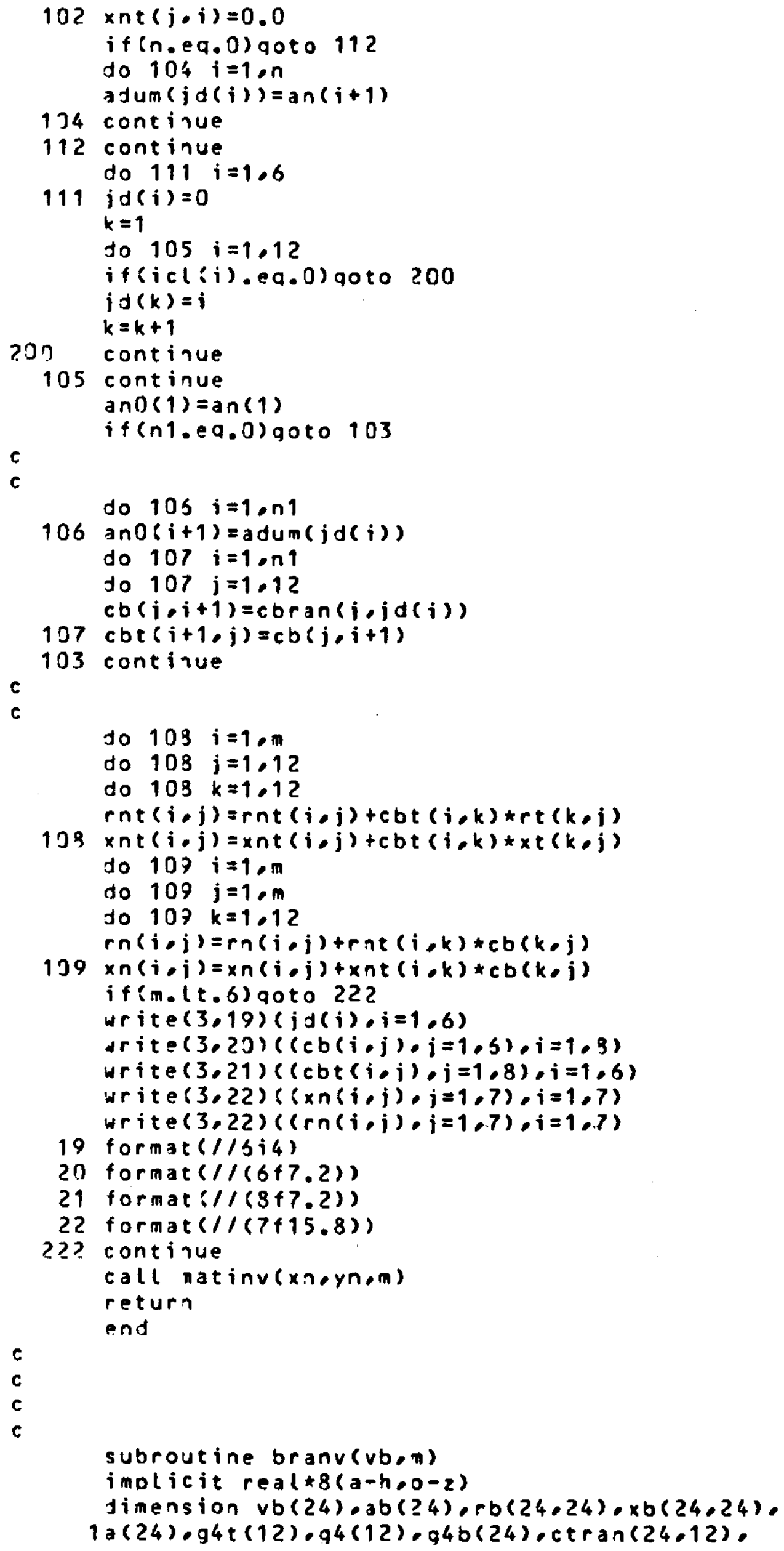




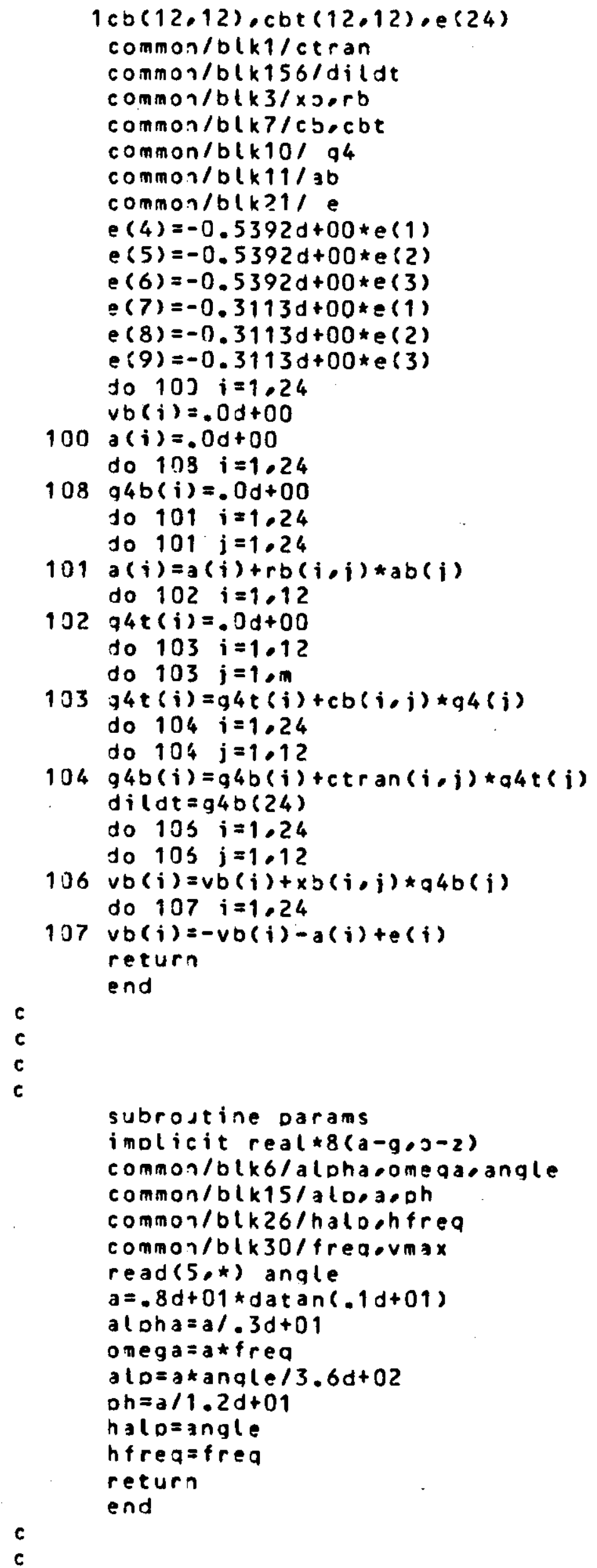


subroutine intero(an,ano,icletbotemem2,n,iv) imolicit real $\star 8(a-h, j-z)$

dimension $v b(24), a n(12), \operatorname{ano}(12), v d(12), v d 0(1$

$12) \cdot i c((12), j d(6), i t r i q(13), v d 5(12)$

commor/blk8/ jd

common/blk12/vdevd0

common/blk13/ int 1,int?

common/blk201/vb

common/blk439/ikeij

common/blk33/itria

common/blk24/tn

common/blk28/intv

commor/blk32/tdet 1

common/blk34/nt

common/blk61/inta

common/blk53/ax

common/blk47/int

in $\mathrm{t}=0$

$a x=0.0$

int $a=0$

$n t=0$

$n i=1$

$n 2=m$

$t v=t$

$t i=t$

call orani(anom)

call branv (vb,m)

call cnduct (vbovd)

call cducts (vonevas)

vdum $1=\operatorname{vd}($ int 1$)$

vdum $2=v d(i n t 2)$

vdum01 $=v d 0(i n t 1)$

vdum $0 ?=v 10($ int 2$)$

$t \vee 1=t$

if (ik.tt.6.and.iv.eq.0) 90 to 997

intva $=$ int 1

to $103 i=1,6$

if $(v d(i) .(e .0 .0 . a n d . v d 5(i) .(e .0 .3)$ goto 200

if (itrig(i).ea.1) qoto 210

if ( (vtum1-vd(i)).qe.0.0) qoto 200

$t 1=(v \operatorname{sum} Q 1-v d j(i)) \star t /(v d u n 01-v d 0(i)+v d(i)-v d u m 1)$

if $(t 1 . g t \cdot t v 1)$ goto 20 J

qoto 200

210 continue

$t v 1=t$ ?

$i k=0$

intva $=i$

200 continue

100 continue

999 continue

$i k=i k+1$

$t \vee 2=t$

if(ij.lt.6.and.iv.ea.0) 90 to 998

intvb=int 2

do $101 i=7,12$

if $(v d(i) . l e \cdot 0.0$ and.vds $(i) . l e .0 .0)$ goto 201

if (itrig(i).eq.1)goto 211

if ( (vjum2-vo(i)).ge.0.0)goto 201 


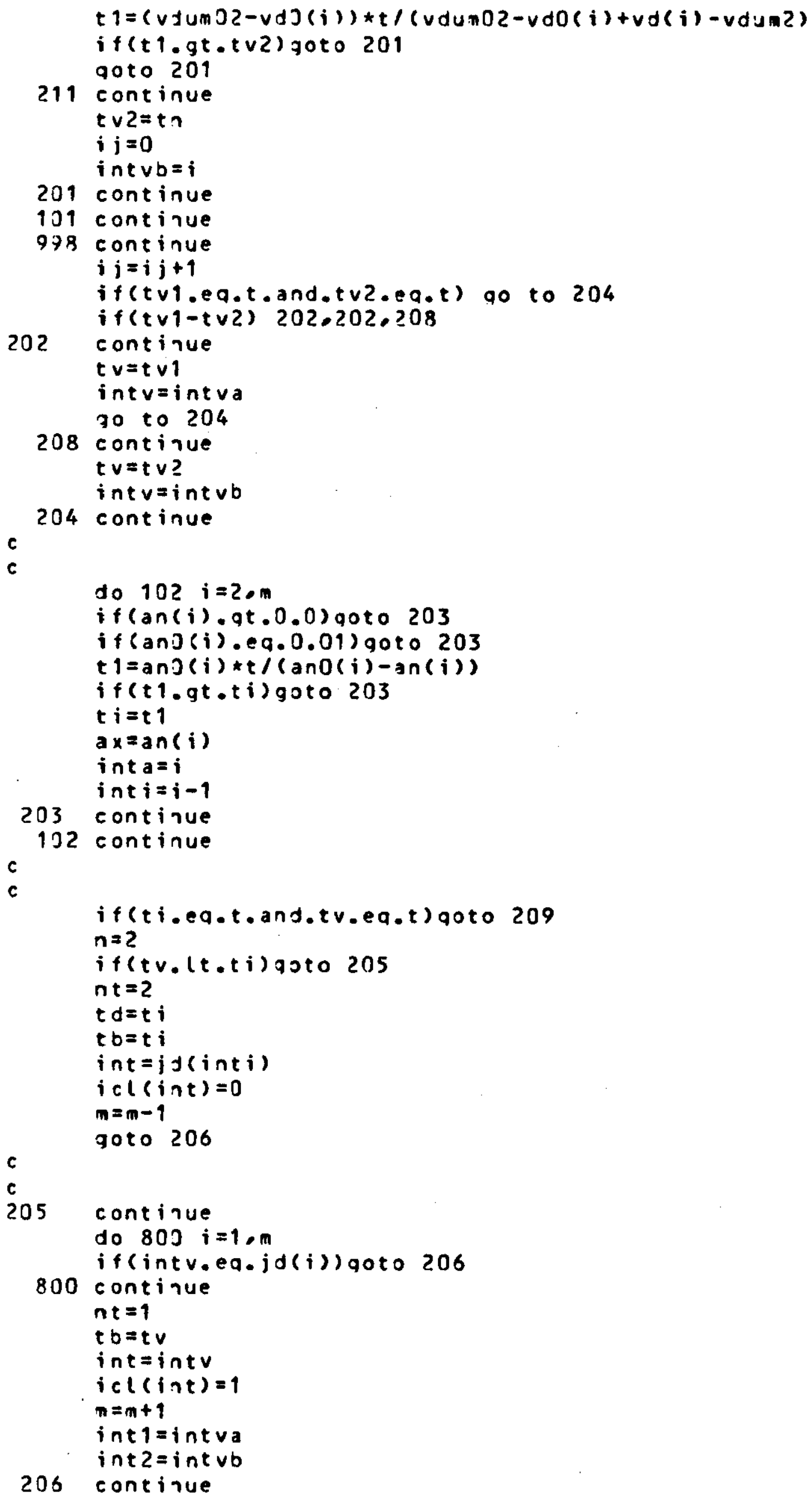




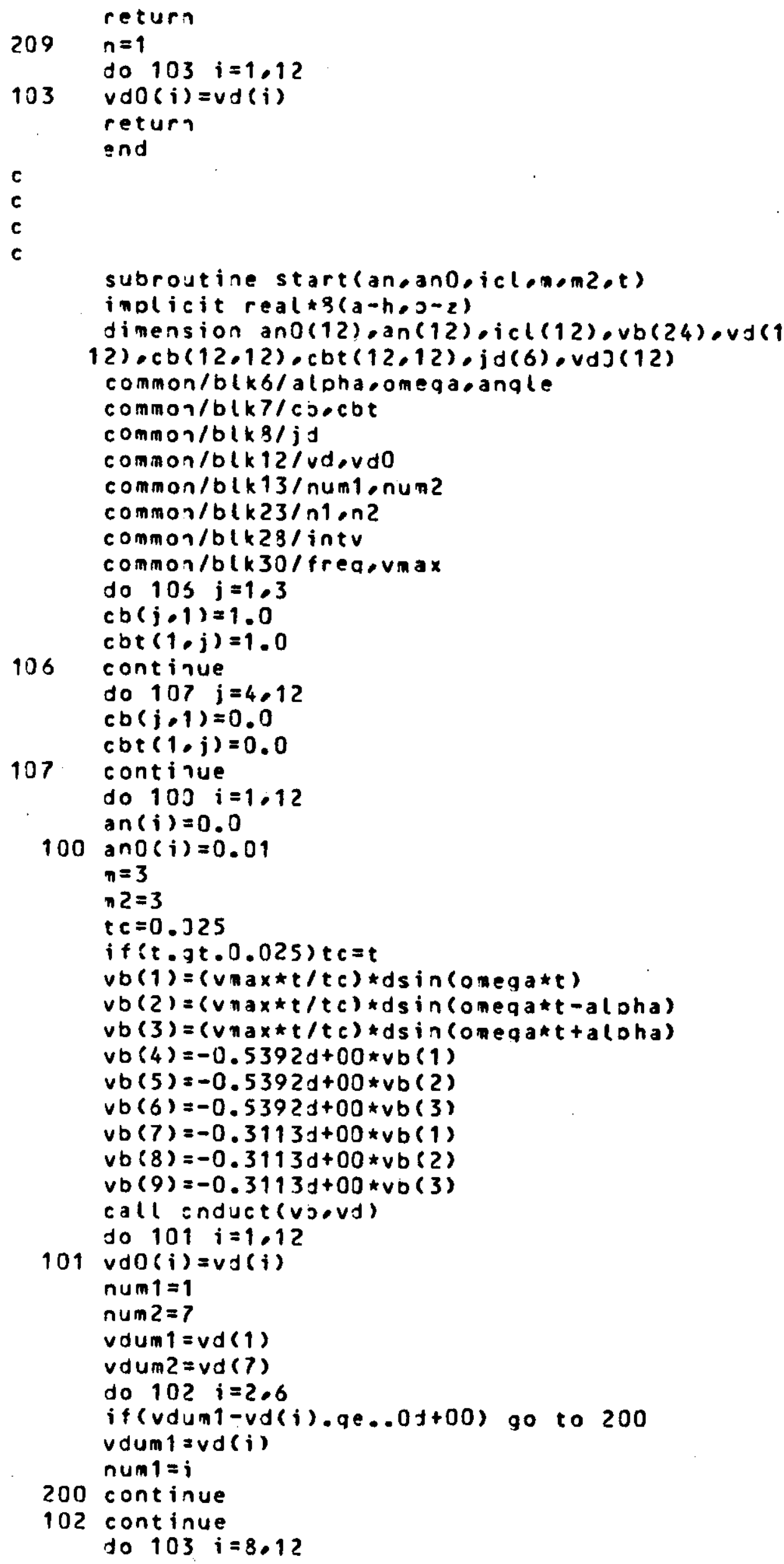


if(vdum2-vd(i).ge..0d+00) 90 to ?01

$v \operatorname{dum} 2=v d(i)$

num $2=i$

$2 J 1$ continue

103 continue

to $104 \quad i=1,12$

$104 i c(c i)=0$

do $105 \quad i=1,6$

$135 \mathrm{j} d(i)=0$

i $c(\operatorname{num} 1)=1$

i $c($ inum 2$)=1$

id $d(1)=$ nun 1

j $d(2)=n u n z$

$n 1=$ nun 1

$n 2=n u n 2$

retur?

end

c

C

c

c

subroutine load ( $m$ )

inolicit real $\star 8(a-9 \cdot 0-2)$

dimension cbran(12,12), ctran(24,12), ctrant (12,2

$14), c b(12,12), c b t(12,12), r n(12,12) \cdot x n(12,12), r t$

$2(12,12), x t(12,12), r b(24,24), \times b(24,24)$,r (

$312,24), x(12,24), j d(6), r a(24,12), x a(24,12), y t(12$

$4.12) \cdot \operatorname{yn}(12,12)$

common/blk1/ctran

common/blk2/cbran

common/blk3/xborb

common/blk4/ytortext

common/olk5/xnerneyn

common/blk $7 / c b$ cbt

common/blks/jd

common/btk9/etrant

commor/blk25/ebethy

common/blk27/hrlehxl, heb

common/blk31/rlexl

do $10 J i=1.24$

do $103 j=1.12$

$100 \operatorname{ctant}(j, i)=\operatorname{ctran}(i, j)$

do $101 i=1, \mathrm{~m}-1$

do $101 j=1,12$

$c b(j \cdot i+1)=c b r a n(j \circ j d(i))$

$101 \operatorname{cbt}(i+1, j)=c b(j-i+1)$

$h r l=r l$

$h \times l=\times l$

he $b=e$ o

call labell

do $102 \quad i=1.24$

do $102 j=1.12$

$r(j, i)=0.0$

$102 \times(j, i)=0.0$

do $103 \quad i=1,12$

do $103 j=1.24$

$r a(j \cdot i)=0.0$

$103 \times a(j, i)=0.0$

do $104 i=1.12$

do $104 j=1.12$ 


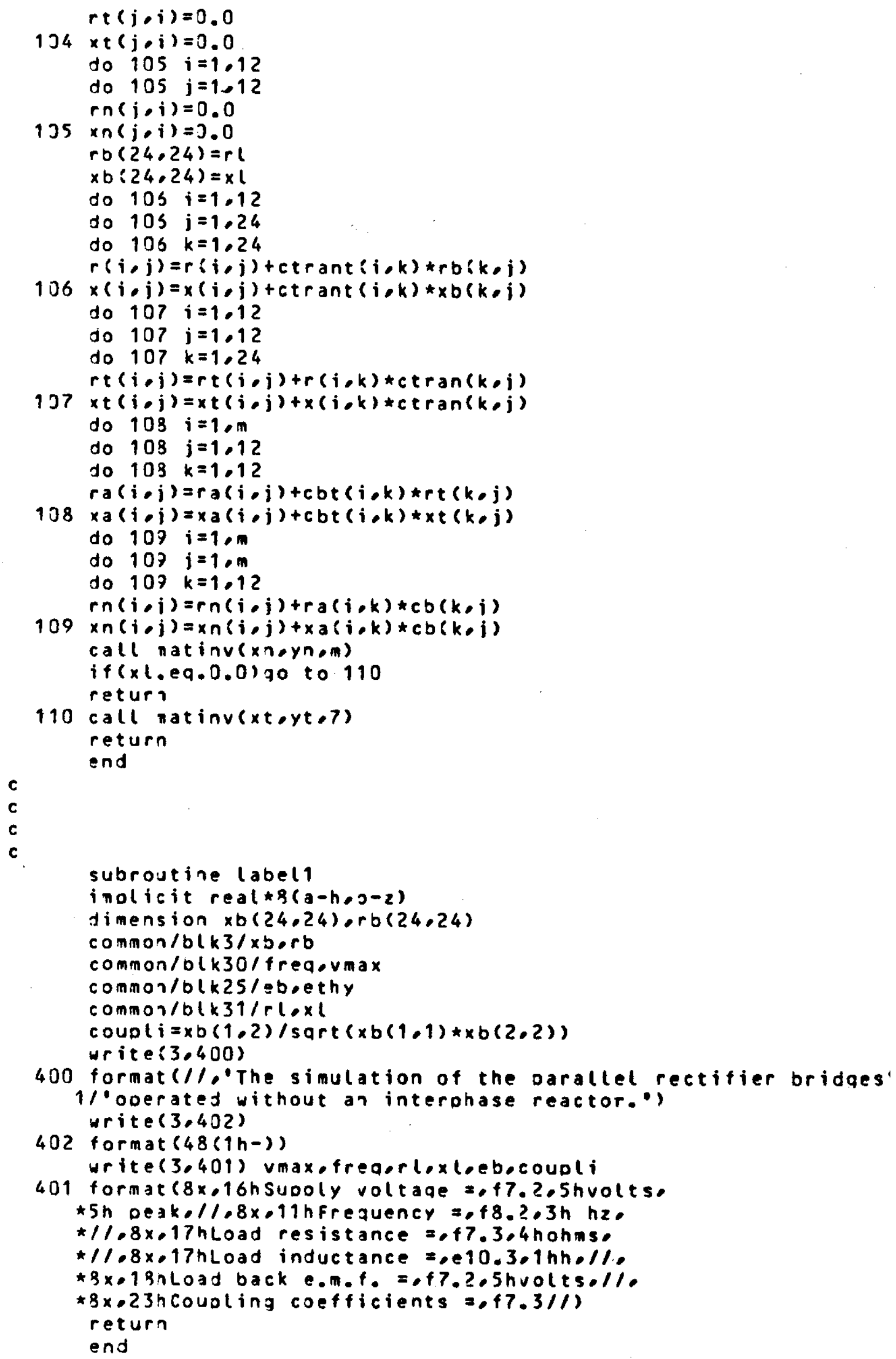

400 format $\left(/ / 0^{\circ}\right.$ The simulation of the darallel rectifier bridaes $1 /$ 'oderated without an interphase reactor.") 
subroutine oostediterodetton)

inolicit real $\star 8(a-h, o-z)$

Jimension $a(3.8), r(8), e(8), x n(12,12) \cdot y n(12,12), r n(12.12)$

common/blks/xnerneyn

do $100 \mathrm{i}=1, \mathrm{~m}$

do $100 j=1, m$

$100 a(j-i)=0.0$

do $101 i=1, m$

do $101 \mathrm{j}=1$, m

do $101 \mathrm{k}=1, \mathrm{~m}$

$101 a(i, j)=a(i, j)+y n(i, k) \star r n(k, j)$

$i f a i l=0$

call f02 aaf (a.8,mer,e,ifail)

if $(i f a i l . e q .0)$ go to 200

write $(3,300)$ ifail

300 format ('error in 102 aafoifail $=0,02$ )

stoo

200 continue

$t 1=\operatorname{de}(t * r(m)$

if $(t 1 . q t .1 .0)$ go to 201

iter $=1$

to to 202

201 iter=nint $(t 1)$

202 continue

steo=delt/iter

write $(3,301)$ steo

301 format('steo lenath =",e12.5)

return

end

c

c

c

subrostine $m \times r k 4(t 1, t$ stopoaneano,neiter)

imolicit real $\star 8(a-h, 0-z)$

dimension vn(12) $\times x n(12.12) \cdot \operatorname{rn}(12.12) \cdot y n(12.12)$

timension $q 0(12) \cdot 91(12) \cdot 92(12) \cdot 93(12) \cdot \operatorname{an}(12)$

$1 \cdot \operatorname{ano}(12) \cdot 74(12)$,atemo $(12) \cdot v(12)$

common/blks/xnorn,yn

common/blk10/g4

common/blk20/vn

niter $=$ float (iter)

$d t=(t s$ too $-t 1) / h i t e r$

$t=t 1$

to $103 i=1$, m

100 an $(i)=\operatorname{an} 0(i)$

Jo $101 i=1$, iter

do $102 j=1 \circ m$

132 atemo( $j)=\operatorname{an}(j)$

call zn(t,an+j0,n)

do $103 \mathrm{j}=1$. $\mathrm{m}$

103 an $(j)=\operatorname{atg}$ eno $(j)+q 0(j) \star d t / 0.2 d+01$

$t=t+d t / 0.2 d+01$

$c a l l$ an $(t, a n \cdot a 1, m)$

do $104 j=1, m$ 
$104 a n(j)=a \operatorname{temo}(j)+a 1(j) \star d t / 0.2 d+01$

call an (t,anคq2,m)

to $105 j=1, n$

105 an $(j)=\operatorname{ateno}(j)+q 2(j) \star d t$

$t=t+d t / 0.2 d+01$

call on $(t, a n \cdot g 3, m)$

do $105 j=1, m$

$106 \operatorname{an}(j)=\operatorname{ateno}(j)+(q 0(j)+0.2 d+01 * q 1(j)+0.2 d+31 * 22(j)+q 3$

$1(j)) * j t / 0.6 d+01$

101 continue

Jo $107 \mathrm{j}=1, \mathrm{~m}$

$v(j)=3.0 d+00$

$137 q 4(j)=0.0 d+00$

to $108 j=1, m$

do $103 k=1, m$

$108 v(j)=v(j)+r n(j, k) \operatorname{an}(k)$

do $107 j=1 \circ m$

$109 v(j)=-v(j)+v n(j)$

do $110 j=1, m$

do $113 k=1, \mathrm{~m}$

$110 \exists 4(j)=94(j)+y n(j \circ k) * v(k)$

return

end

c

subroutine gn(toanogคn)

implicit real $(8)(a-h, 0-2)$

dimension an (12) , rn (12.12) $x$ nn(12.12),yn(12.1

12) $, q(12) \cdot v b(24), v t(12), v n(12), c t r a n t(12.24)$

$1 \cdot c b(12,12), c b t(12,12), d(12)$

common/blk5/xnerneyn

common/olk6/aloha,omeqaeanqle

common/blk?/cbocbt

common/blkg/ctrant

common/blk20/vn

common/blk21/ vb

common/blk25/ebeethy

comon/blk30/frea,vmax

do $99 \quad i=1.24$

$99 v b(i)=0.0 a+00$

$t c=0.325$

if $(t \cdot 7 t .0 .025) t c=t$

$v b(1)=(v \operatorname{nax} t t / t c) * d \sin ($ oneqa*t)

$v b(2)=(v n a x * t / t c) * d \sin (o n e q a * t-a(o h a)$

$v b(3)=i v m a x * t / t c) \star d \sin (o m e g a * t+a(p h a)$

do $98 \quad i=10,21$

98 vb $(i)=-$ ethy

vo $(24)=-e b$

do $100 \quad i=1,12$

$100 v t(i)=0.0 d+00$

to $101 i=1.12$

do $101 j=1.24$

$101 v t(i)=v t(i)+\operatorname{ctant}(i, j) * v b(j)$

do $102 \quad i=1, m$

$102 \vee n(i)=0.0 d+00$

do $103 i=1, m$

do $103 j=1.12$

$103 v n(i)=v n(i)+c b t(i, j) * v t(j)$ 


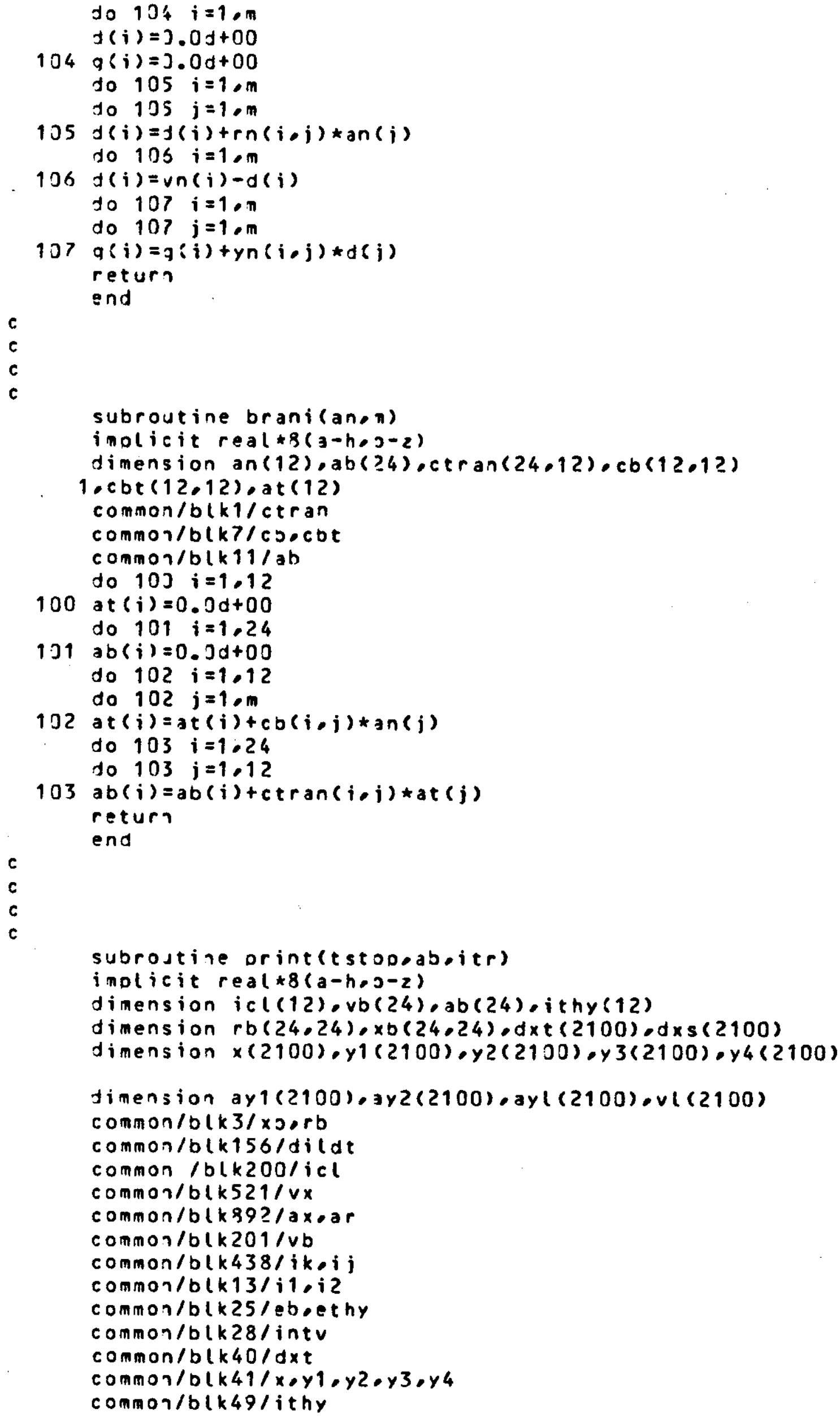


common/blk60/ay1, ay2, ayl,ve

$c$

to $401 \quad i=1.12$

$i \operatorname{thy}(i)=3$

$i o=i+1$

if (io.eq.7) io $=1$

if $(i 0 . e q .13)$ io $=7$

401 continue

c

C

ifficl(7).ea.1.or.icl(8).ea.1) qo to 500

if(icl(9).eq.1.or.icl(10).eq.1) go to 501

if(icl(11).eq.1.or.icl(12).ea.1) yoto 502

qo to 503

$502 v x=v b(7)-v b(9)$

To to 503

$501 v x=v v(7)-v b(8)$

70 to 503

$500 v x=-0.6$

503 continue

$a x=a b(4)-a b(5)$

$3 r=a b(1)-a b(3)$

if(tstop.le.0.1050.0r.tstop.3t.0.1250)return

$x(i t r)=t s t 00-0.1050$

if $(i t r . e q .1)$ qoto 10

$d x t(i t r)=x(i t r)-x(i t r-1)$

if $(d x t(i t r) \cdot(t .0 .9 d-37) r e t u r n$

qoto 11

$10 \mathrm{dxt}(1)=x(1)$

11 continue

$y 1(i t r)=a x$

$y 2(i t r)=a b(7)$

$y 3(i t r)=a b(4)$

$y 4(i t r)=a r$

ay $1(i t r)=a b(23)$

ay $2(i t r)=a b(22)$

$a y($ (it $r)=a b(24)$

$v(i i t r)=a b(24) * r b(24,24)+d i(d t * x b(24,24)+e b$

c writg(3,400)x(itr),dxt(itr),ab(1),ar,ax,aj(7),vx,v(l(itr),i1,i2

*icl( 1$)$, icl( 2$)$, icl (3), icl (4), ict (5).

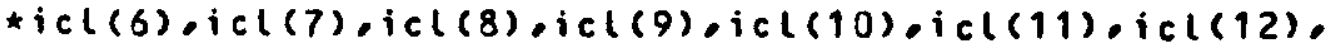

*ithy (1), ithy(2), ithy(3),ithy(4),ithy(5), ithy (6),

*ithy (7), ithy (8), ithy (9), ithy (10), ithy (11), ithy (12)

400 format $(+9.5 .+9.6 .2+15.11 .4(1 x+f 7.2) \cdot 2(1 x+i 2) \cdot 1 x+12(1 x+i 1) \cdot 1 x$. $114(1 x+i 1))$

it $r=i t r+1$

return

end

c

c

c

subroutine matinv(xindeyindena)

imolicit real $\star 8(a-h, 0-z)$

dimension $x$ int $(12,12)$.yind $(12,12)$, aug $(12,24)$

do $50 J i=1$, na

do $500 j=1$, na

$500 i f(\operatorname{abs}(x \operatorname{ind}(j, i)) .(t .1 .0 d-08)$ xind $(j, i)=0.0 d+00$

to $281 i=1$ ona 


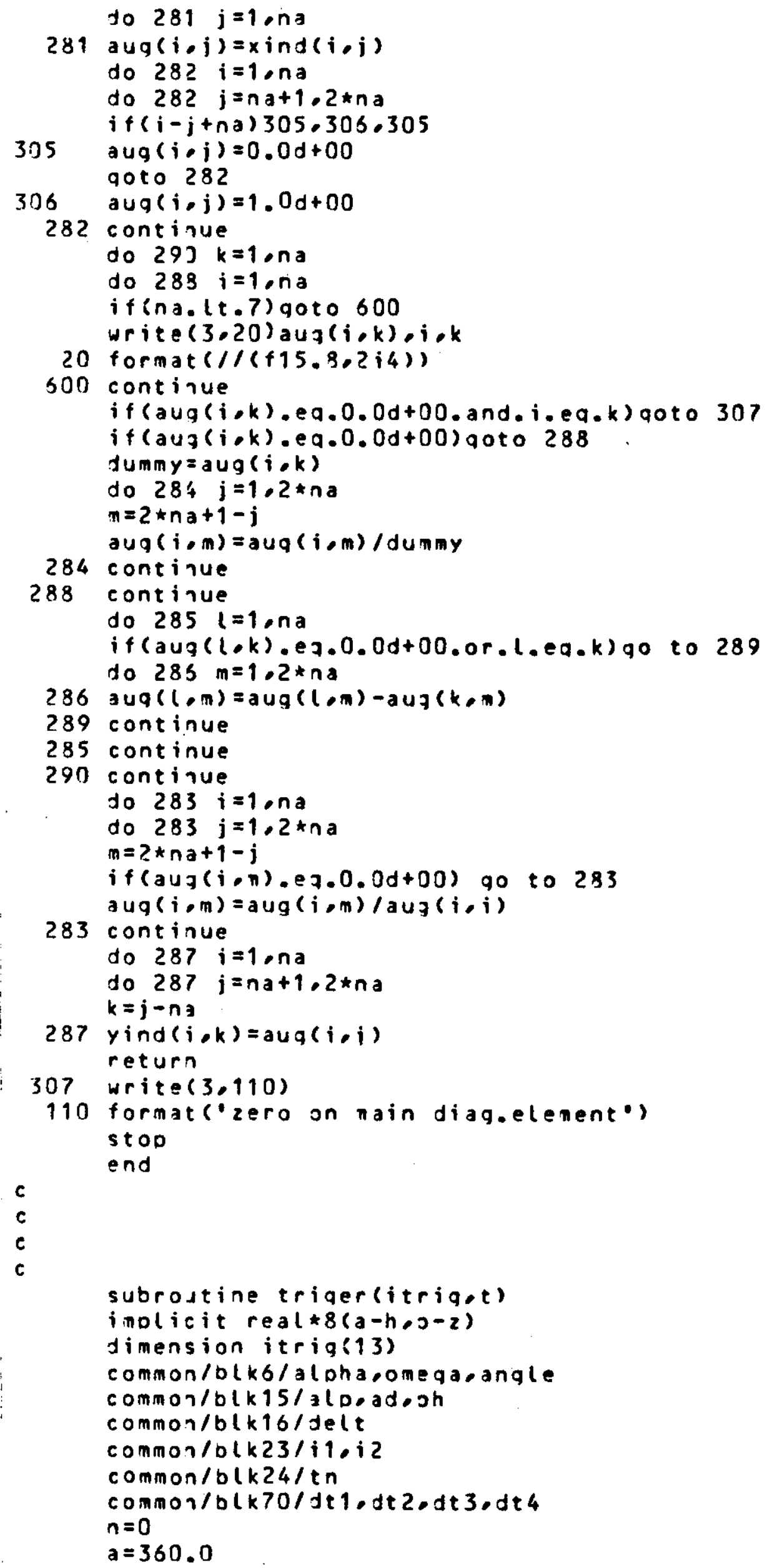




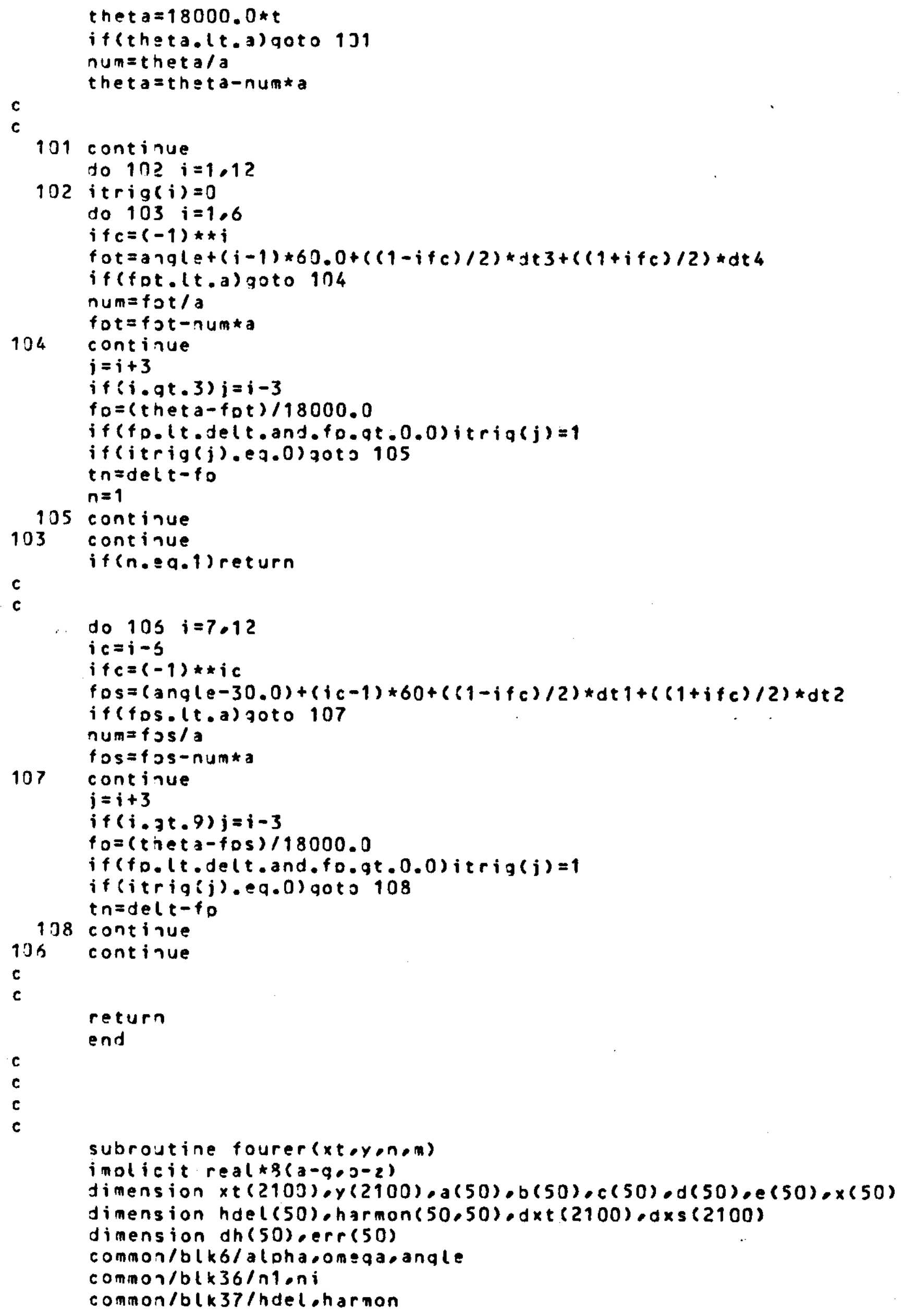




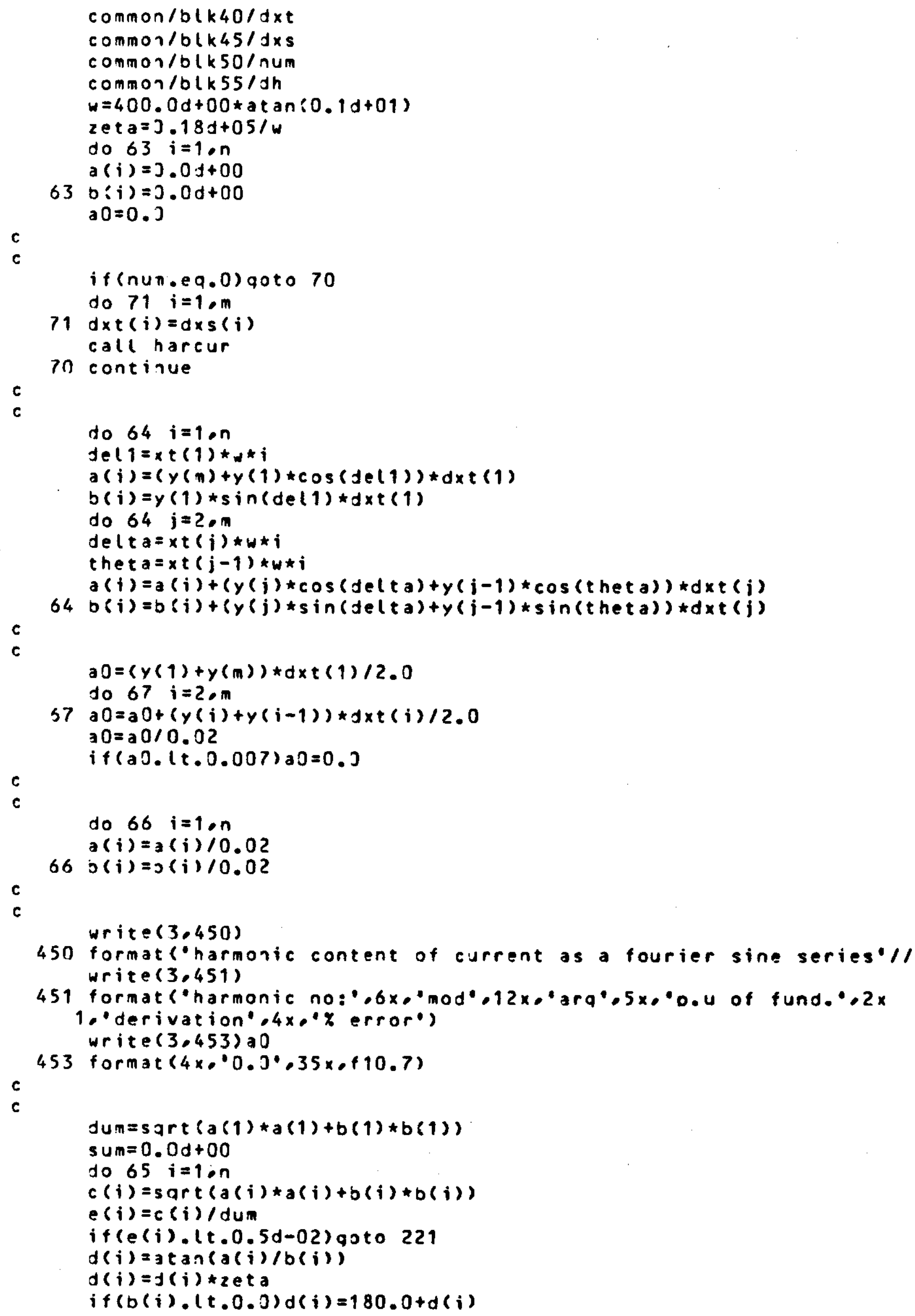

450 format ("harmonic content of current as a fourier sine series $/ /$ write (3,451)

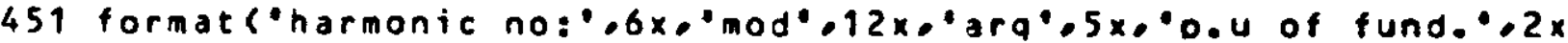
1. "derivation' $4 \times 0^{\circ} x$ error $\left.^{\circ}\right)$ write $(3,453)$ a 0 


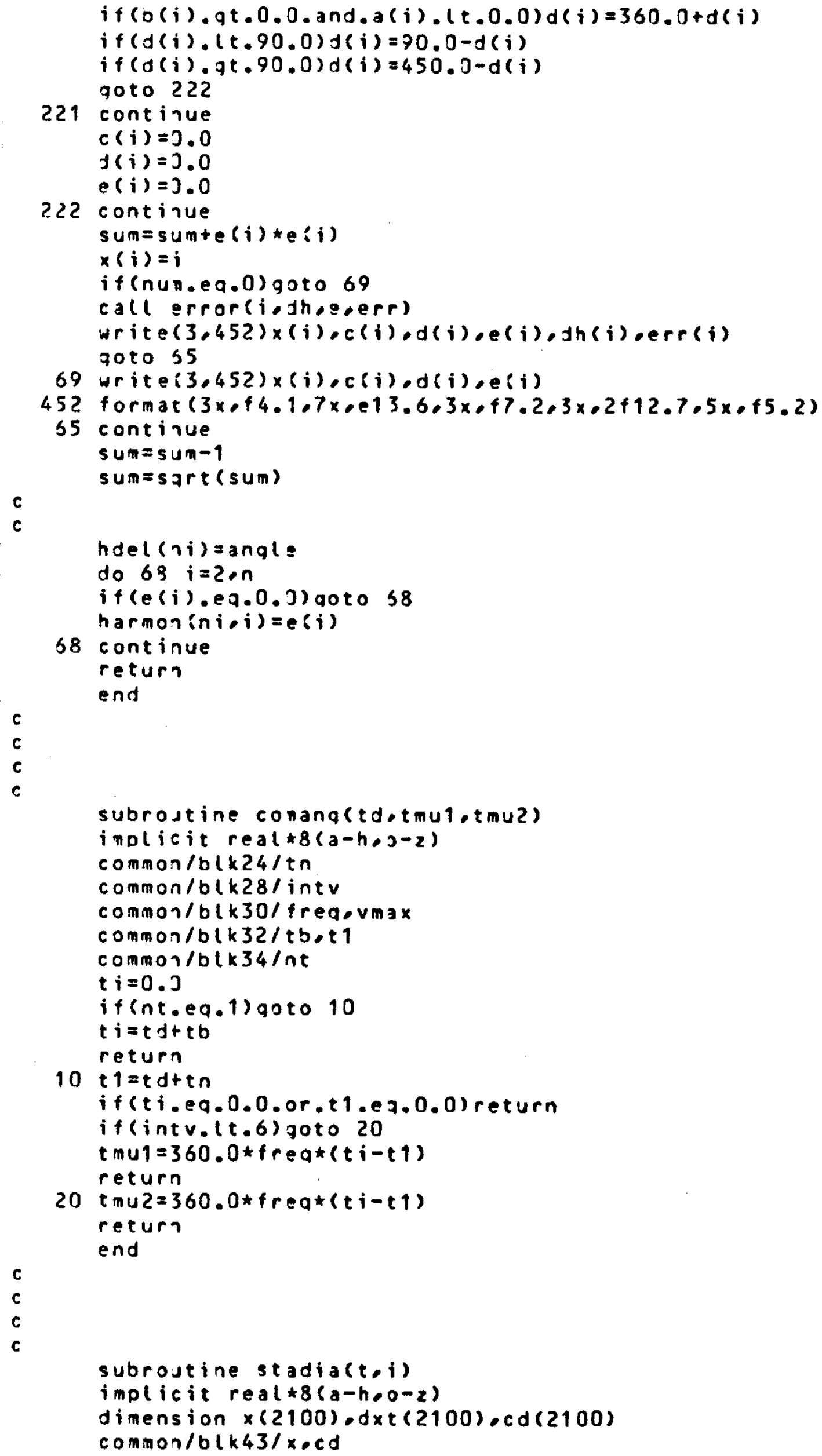




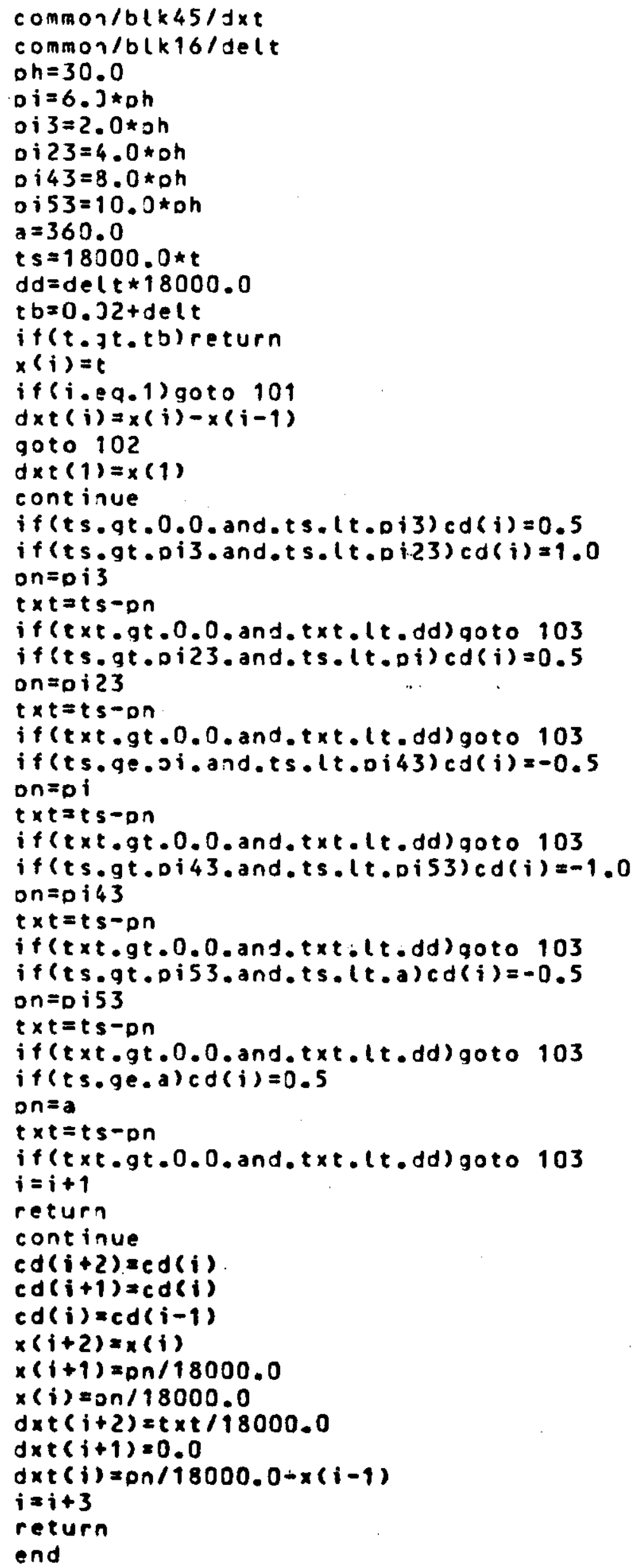


subroutine charac

real $\star 8$ tileti2,to1,to2

real $* 3$ cilecizocol,col

real $\star 3$ ynl $(30), y m 2(3)), y n((3)), r((30)$

dimension $h(50.50)$.delay $(50)$

dimension $t i 1(30) \cdot t i 2(30) \cdot \operatorname{tol}(30) \cdot \operatorname{to} 2(30) \cdot \bmod 1(30) \cdot \bmod 2(30)$

dimension cil $(30), c i 2(30) \cdot \operatorname{col}(30) \cdot \cos (30)$

common/blk36/noi

common/blk37/delayeh

common/blk46/ti1.ti2.t01.to2

common/blk48/mod1, mod2

common/blk51/ci1.ci2.c01.co2

common/blk66/ymi,ym2,ymlerl:

write $(3,10)$

write $(3,11)$

10 format $\left(10 x 0^{-T h e}\right.$ bridge supply line current harmonic contents ${ }^{\circ}$ $110 x \cdot 53(1 h-) /)$

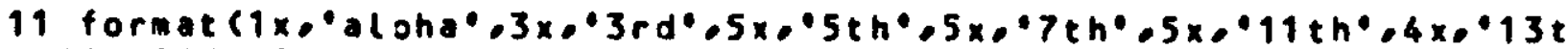

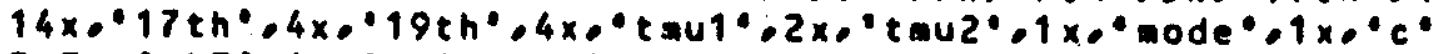

$2.3 x_{0} \cdot \operatorname{th} 7^{\circ}, 4 x_{0} \cdot \operatorname{th} 1 \cdot .5 x_{0} \cdot i d 1^{\circ} .6 x_{0} \cdot i d 2 \cdot .6 x_{0} \cdot i(\cdot 1)$

do $100 j=1$, n

ngte $=0$

if(ti1(j).tt.to1(j).or.ti2(j).te.to2(j))goto 13

$t 01(j)=t 01(j)+0.02$

$t 02(j)=t 02(j)+0.02$

13 continue

if (most(j).eq.3)tmul $=(t 01(j)-t i 1(j)) \star 18000.0$

if $(\bmod 2(i) \cdot e q .3) t \operatorname{mu} 2=(\operatorname{to} 2(j)-t i 2(j)) \star 18000.0$

if $(\bmod 1(j) \cdot e q \cdot 2 \cdot \operatorname{or} \cdot \bmod 1(j) . e q .4) t \operatorname{mu} 1=(\operatorname{to} 2(j)-t i 1(j)) * 18000.0$

if $(\bmod 2(j) . e q .2 .0 r \cdot \bmod 2(j) \cdot e q .4) t \operatorname{mu} 2=(t 01(j)-t i 2(j)) * 18000.0$

if $(\operatorname{moj} 2(j) . e q .2$. and. $t$ nuz.$q t .50 .0) t$ muz $=t$ muz -63.0

if $(\operatorname{mos} 1(j) \cdot e q \cdot \bmod 2(j))$ goto 17

if $(\bmod 1(j) \cdot g t \cdot \bmod 2(j)) g 0 t 018$

if $(\bmod 1(j) . \in a .3)$ goto 19

tmode $=2.3$

$\operatorname{tmu} 1=(t i 1(j)-t 01(j)) * 18000.0$

$\operatorname{th} 7=(t 01(j)-\operatorname{ci}(j)) * 18000.0$

$\operatorname{th} 1=(\cos (j)-t i 2(j)) \star 18000.0$

70 to 20

18 continue

if $(\bmod 1(j) \cdot e a \cdot 3)$ goto 19

$t \operatorname{mode}=4.3$

$\operatorname{tmu} 2=30.0+(t i 2(j)-t 01(j)) * 18000.0$

$\operatorname{th} 7=(\operatorname{col}(j)-c i 1(j)) \star 19000,0$

$\operatorname{th} 1=(\operatorname{col}(j)-c i 2(j)) \star 18000.0$

qoto 20

19 write $(3,300)$ delay $(j)$

300 format $(7.2 .13 \times$. This operation is not expected for the model" goto 100

17 continue tmode $=\bmod 1$ ( $j)$

goto $(14 \cdot 15 \cdot 16 \cdot 16) \cdot \bmod (i)$

14 continue

$t h 7=(t 01(j)-t i 1(j)) \star 18000.0$

$t h 1=(t 02(j)-t i 2(j)) \star 18000.0$

goto 20

continue 
$\operatorname{th} 7=(t 01(j)-c i 1(j)) \star 18000.0$

$\operatorname{th} 1=(\operatorname{to} 2(j)-\operatorname{ti2}(j)) * 18000.0$

$i f(t h 7.9 t .90 .0) \operatorname{th} 7=t h 7-60.0$

goto 20

16 continue

$\operatorname{th} 7=(\operatorname{col}(j)-\operatorname{ci}(j)) * 18000.0$

$\operatorname{th} 1=(\cos (j)-\operatorname{ci} 2(j)) \star 18000.0$

20 continue

if $(t \operatorname{mu} 1 . t t \cdot 0.1$. and.modi (j). gt. 1 ) ngte $=1$

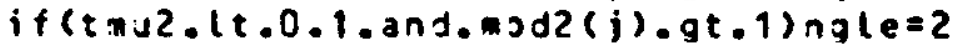

write $(3,200)$ delay $(j), h(j, 3), h(j, 5), h(j, 7), h(j, 11), h(j, 13)$

$1, h(j, 17), h(j, 19), t m u 1 \cdot t m u z, t$ mode.ngle,th7,thl,ym1(j),ym2(j),ynt

200 format $(f 7.2 .7+8.5 .2+6.2 .+4.1 . i 3.2 f 7.2 .3(1 \times .+3.2))$

100 continue

return

end

C

c

6

c

subroutine harcur

inolicit real $* 8(a-h, 0-2)$

dimension $h(50)$

common/blk5s/h

do $20 n=1.50$

$20 h(n)=0.0$

$0 i=4 . j * a \tan (1.0)$

$a=2.0 / 0 i$

hdum $=3.0 / 0 i$

$h(1)=1.0$

do $21 \quad i=1.24$

$n=2 \star i+1$

on $=n$

pn $1=p n * p i / 2.0$

$p n 2=p n \star p i / 6.0$

$b=\sin (\operatorname{on} 1)+\sin (p \cap 2)$

$h(n)=a * b /(p n \star h d u m)$

21 continue

return

end

subroutine error(i,dhoeeerr)

imolicit real $\star 8(a-h, 0-z)$

dimension dh $(50)$,e $(50)$ eerr $(50)$

if $(d h(i)$ eq.0.0.or.e(i).ea.0.j)goto 10

er $=(\operatorname{abs}(d h(i))-a b s(e(i))) / a b s(d h(i))$

$\operatorname{err}(i)=a b s(e r) \star 100.0$

return

$10 \operatorname{err}(i)=0.0$

end

subrostine extrap(tstopetioan)

inolicit real $\star 8(a-h, 0-2)$

common/blk53/ax 


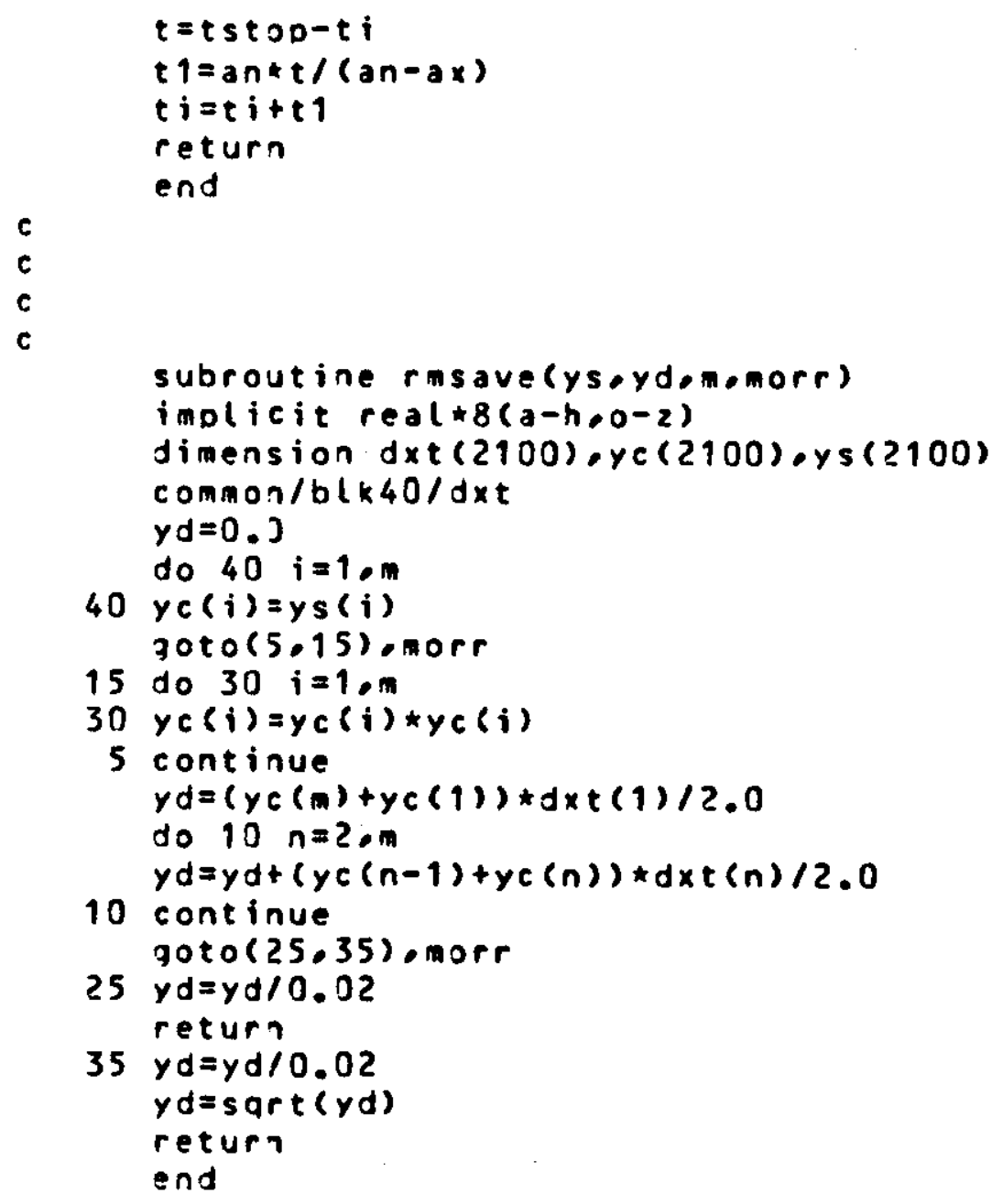




\section{$\underline{\text { DATA-DIMM }}$}

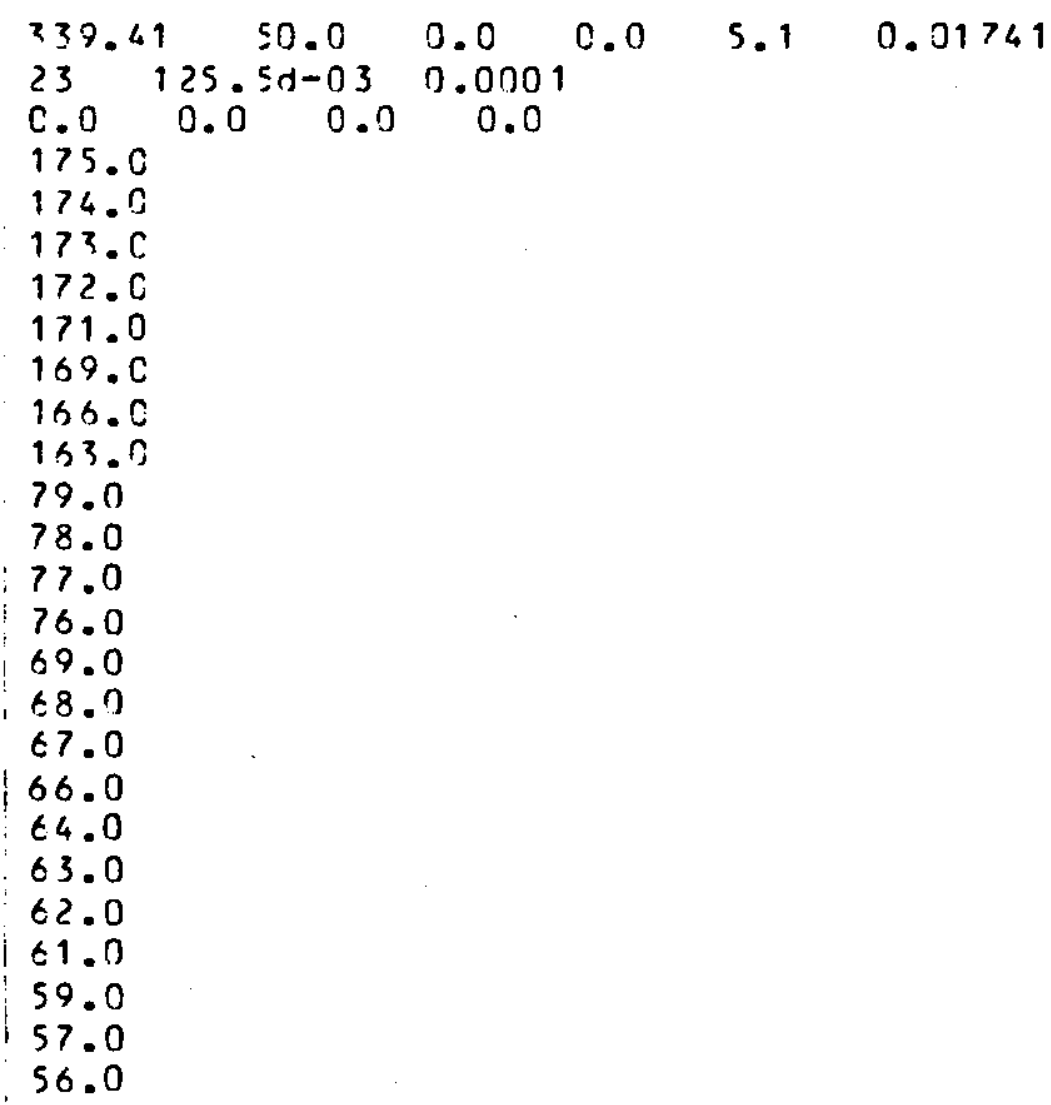




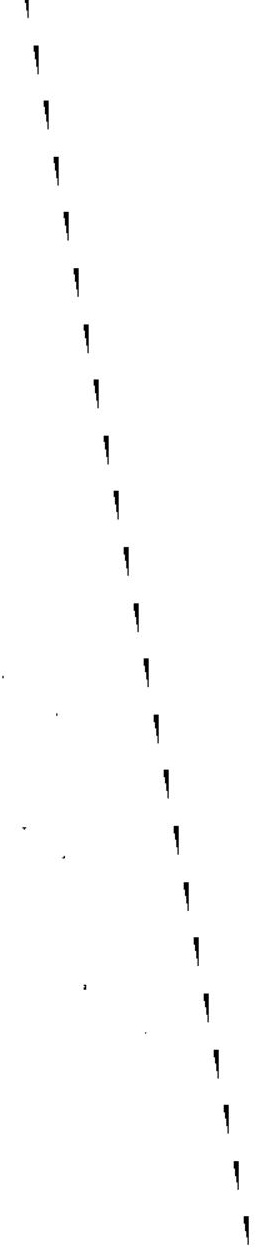

